NOV 1 a 1991

\title{
LIQUID MEMBRANE PURIFICATION OF BIOGAS
}

DISTRIBUTION OF THIS DOCUMENT IS UNLIMITED 
The New York State Energy Research and Development Authority (Energy Authority) is responsible for the development and use of safe, dependable, renewable and economic energy sources and concervation technologies. It sponsors energy research, development and demonstration (RD\&D) projects and financing programs designed to help utilities and other private companies fund certain energy-related projects. The Energy Authority is a public benefit corporation which was created in 1975 by the New York State Legislature.

In working toward these goals, the Energy Authority sponsors research, development and demonstration projects in two major program areas: Energy Efficiency and Economic Development, and Energy Resources and Environmental Research.

Under its financing program, the Energy Authority is authorized to issue tax-exempt bonds to finance certain electric or gas facilities and special renergy projects for private companies.

The Energy Authority also has responsibility for constructing and then operating facilities for the disposal of low-level radioactive wastes produced in New York State. The gerierators of these wastes ultimately will bear the costs of the construction of these facilities.

A 13-member board of directors governs the Energy Authority, with William D. Cotter, Commissioner of the State Energy Office, serving as Chairman of the Board and Chief Executive Officer. Irvin L. White, President of the Energy Authority, manages its programs, staff and facilities.

The Energy Authority derives its basic RD\&D revenues from an assessment levied on the intrastate sales of New York State's investor-owned electric and gas utilities. Additional RD\&D funds come from the investment of retained earnings, as well as from an annual contribution from the New York Power Authority.

The Energy Authority's RD\&D program is also supported by funds from a variety of cosponsors, including utilities, universities, industries, private engineering and scientific research firms, local governments, and State and Federal agencies.

For further information on the Energy Authority's programs, contact the Department of Communications, New York State Energy Research and Development Authority, phone (518) 465-6251, extension 272.

State of New York

Mario M. Cuomo,

Governor
Energy Rasearch and Development Authority

William D. Cotter,

Chairman

Irvin L. White,

President 
THE NEW YORK STATE

\section{ENERGY RESEARCH AND DEVELOPMENT AUTHORITY}

Project Manager

Jeffrey M. Peterson

and

THE NEW YORK GAS GROUP

Project Manager

Charles C. Hong

and

THE GAS RESEARCH INSTITUTE

\section{Project Manager \\ Howard Meyer}

Prepared by

STEVENS INSTITUTE OF TECHNOLOGY

Department of Chemistry and Chemical Engineering

Fifth and River Streets

Castle Point Station

Hoboken, New Jersey 07030

Project Director

Professor K. K. Sirkar

Investigators

Dr. S. Majumciar

Dr. A. K. Guh.

Dr. Y. T. Lee

T. Papadopoulos

S. Khare

663-RIER-NCRB-84

Energy Authority

Report 91-9 


\section{NOTICE}

This report was prepared by the Stevens Institute of Technology in the course of performing work contracted for and sponsored by the New York Gas Group, the Gas Research Institute and the New York State Energy Research and Development Authority, (hereafter the "Sponsors"). The opinions expressed in this report do not necessarily reflect those of the Sponsors or the State of New York, and reference to any specific product, service, process, or method does not constitute an implied or expressed recommendation or endorsement of it. Further, the Sponsors and the State of New York make no warranties or representations, expressed or implied, as to the fitness for particular purpose or merchantability of any product, apparatus, or service, or the usefulness, completeness, or accuracy of any processes, methods, or other information contained, described, disclosed, or referred to in this report. The Sponsors, the State of New York, and the contractor make no representation that the use of any product, apparatus, process, method, or other information will not infringe privately owned rights ana will assume no liability for any loss, injury, or damage resulting from, or occurring in connection with, the use of information contained, described, disclosed, or referred to in this report.

First Printing: October 1991 


\section{CONTENTS}

EXECUTIVE SUMMARY S-1

1 INTRODUCTION $1-1$

2 MODELING AND SIMULATION $2-1$

Analysis of Gas Mixture Separation in a HFCLM Permeator

Sweep Gas Mode of Operation 2-2

Conventional or Vacuum Mode of Operation 2-7

Sweep Water Mode of Operation $2-10$

Determination of Effective Membrane Thickness in a HFCLM Permeator

Determination of Permeability of Nonreactive Species through an Aqueous Solution of DEA

Analysis of Facilitated Transport of $\mathrm{CO}_{2}$ through an Aqueous Solution of DEA

Simulation Techniques for Separation Problems in HFCLM Permeator

Solution Procedure for Facilitated Transport Analysis

Generalized Two Dimensional Model for Membrane Thickness Determination

Determination of Wettability, Bubble Points and Breakthrough Points

Fabrication of Small Scale HFCLM Permeators 3-2

Fabrication of Large Scale HFCLM Permeators 3-10

Permeator Testing Under Static Pressure 3-15

Description of Purification Loop for Various Modes in $\begin{array}{lr}\text { Small Scale Operation } & 3-17\end{array}$

$\begin{array}{lr}\text { Sweep Gas Mode } & 3-17\end{array}$

Conventional Polymeric Membrane Moder 3-22 
Vacuum Mode

Sweep Water Mode

Description of the Larger Purification Loop

Experimental Procedure

Measurement of Gas Permeability through the Liquid Membrane

Calibration and Analysis

Estimation of Pure Component Gas Permeabilities through Water

Estimation of Pure Component Gas Permeabilities through Aqueous Solutions of DEA

Estimation of Effective Membrane Thickness in Small Permeators

Purification Studies of Model Biogas Mixtures in Sweep Gas Mode through Different Liquid Membranes in Sinali Scale Operation

Water as Liquid Membrane and $40 \% \mathrm{CO}_{2}-60 \% \mathrm{~N}_{2}$ as Feed Gas

Water as Liquid Membrane and $40 \% \mathrm{CO}_{2}-60 \% \mathrm{CH}_{4}$ as Feed Gas

$30 \mathrm{wt} \% \mathrm{~K}_{2} \mathrm{CO}_{3}$ Solution as Liquid Membrane and $40 \% \mathrm{CO}_{2}-$
$60 \% \mathrm{~N}_{2}$ as Feed Gas

Aqueous Solution of DEA as Liquid Membrane and $40 \% \mathrm{CO}_{2}-$ $60 \% \mathrm{~N}_{2}$ as Feed Gas

Aqueous Solution of $20 \mathrm{wt} \%$ DEA as Liquid Membrane and $40 \% \mathrm{CO}_{2}-60 \% \mathrm{CH}_{4}$ as Feed Gas

Aqueous Solution of $10 \mathrm{wt} \%$ DEA as Liquid Membrane and $5 \% \mathrm{CO}_{2}-95 \% \mathrm{~N}_{2}$ as Feed Gas

Aqueous Solution of NMP as Liquid Membrane and $40 \% \mathrm{CO}_{2}-$ $60 \% \mathrm{~N}_{2}$ as Feed Gas

Purification Studies of Model Biogas Mixtures in Conventional Polymeric Membrane Mode with Different Liquid Membranes

Water as Liquid Membrane and $40 \% \mathrm{CO}_{2}-60 \% \mathrm{~N}_{2}$ as Feed Gas 
$30 \mathrm{wt} \% \mathrm{~K}_{2} \mathrm{CO}_{3}$ Solution as Liquid Membrane and $40 \% \mathrm{CO}_{2}$

- $60 \% \mathrm{~N}_{2}$ as Feed Gas

Purification Studies of Model Biogas Mixtures under Vaclium Mode of Operation

Water as Liquid Membrane and $40 \% \mathrm{CO}_{2}-60 \% \mathrm{~N}_{2}$ as Feed Gas

Water as Liquid Membrane and $40 \% \mathrm{CO}_{2}-60 \% \mathrm{CH}_{4}$ as Feed Gas

7 wt\% $\mathrm{K}_{2} \mathrm{CO}_{3}$ Solution as Liquid Membrane and $40 \% \mathrm{CO}_{2}-$ $60 \% \mathrm{~N}_{2}^{2}$ as Feed Gas

Purification Studies of Model Biogas Mixtures in Sweep

Water Mode through Different Liquid Membranes

4-103

Water as Liquid Membrane and $40 \% \mathrm{CO}_{2}-60 \% \mathrm{~N}_{2}$ as Feed Gas

4-103

Water as Liquid Membrane and $40 \% \mathrm{CO}_{2}-60 \% \mathrm{CH}_{4}$ as Feed Gas

$4-112$

30 wt\% $\mathrm{K}_{2} \mathrm{CO}_{3}$ Solution as Liquid Membrane and $40 \% \mathrm{CO}_{2}-$ $60 \% \mathrm{~N}_{2}{ }^{2}$ as Feed Gas

$4-118$

$30 \mathrm{t} \% \mathrm{~K}_{2} \mathrm{CO}_{3}$ Solution as Liquid Membrane and $40 \% \mathrm{CO}_{2}-$ $60 \% \mathrm{CH}_{4}^{2}$ as Feed Gas

4-118

20 wt\% DEA Solution as Liquici Membrane and $40 \% \mathrm{CO}_{2}-$ $60 \% \mathrm{~N}_{2}$ as Feed Gas

$4-118$

Estimation of Effective Membrane Thickness in Large Permeators

Purification Studies of Model Biogas Mixtures in Large Scale Operation

Water as Liquid Membrane and $\mathrm{CO}_{2}-\mathrm{N}_{2}$ Gas Mixture as Feed

$20 \mathrm{wt} \%$ DEA Solution as Liquid Membrane and $\mathrm{CO}_{2}-\mathrm{N}_{2}$ Gas Mixture as Feed

20 wt\% DEA Solution as Liquid Membrane and $\mathrm{CO}_{2}-\mathrm{CH}_{4}$ Gas Mixture as Feed

Best Performances under Different Modes of Operation

6 INTERACTIONS WITH CORPORATIONS REGARDING HFCLM TECHNOLOGY 


\section{EXECUTIVE SUMMARY}

Conventional gas purification technologies are highly energy intensive. They are not suitable for economic removal of $\mathrm{CO}_{2}$ from methane obtained in biogas due to the small scale of gas production. Membrane separation techniques on the other hand are ideally suited for low gas production rate applications due to their modular nature. Polymeric membranes as well as liquid membranes have been investigated earlier for biogas purification. Polymeric membranes suffer, in general, from low values of selectivities and permeabilities. Although liquid membranes possess a high species permeability and selectivity, they have not been used for industrial applications due to the problems of membrane stability, membrane flooding and poor operational flexibility, etc. A new hollow-fiber-contained liquid membrane (HFCLM) technique has been developed recently. This technique overcomes the shortcomings of the traditional imnobilized liquid membrane technology.

The new technique uses two sets of hydrophobic, microporous hollow fine fibers, packed tightly in a permeator shell. The inter-fiber space is filled with an aqueous liquid acting as the membrane. The feed gas mixture is separated by selective permeation of a species through the liquid from one fiber set to the other. The second fiber set carries a sweep stream, gas or liquid, or simply the permeated gas stream.

The objectives of the present investigation were as follows.

1. To study the selective removal of $\mathrm{CO}_{2}$ from a model biogas mixture containing $40 \% \mathrm{CO}_{2}$ (the rest being $\mathrm{N}_{2}$ or $\mathrm{CH}_{4}$ ) using a HFCLM permeator under various operating modes that include sweep gas, sweep liquid, vacuum and conventional permeation.

2. To develop a mathematical model for each mode of operation.

3. To build a large-scale purification losp and large-scale permeators for model biogas separation and to show stable performance over a period of one month.

Extensive purification studies with a model $40 \% \mathrm{CO}_{2}-60 \% \mathrm{~N}_{2}$ feed mixture demonstrated excellent purification capabilities of HFCLM permeator under various operating modes. Sweep gas mode of operation with 20 wt\% DEA as a 
liquid membrane yielded a purified gas stream containing only $6 \% \mathrm{CO}_{2}$ with a $\mathrm{N}_{2}$ lecovery of $95 \%$. The amount of sweep helium gas permeation into the product stream was very low, suggesting that air might be used as a sweep gas in a commercial application. The permeability of $\mathrm{N}_{2}$ or $\mathrm{O}_{2}$ being lower than that of helium, the contamination of the product stream (especially with respect to $0_{2}$ ) would be very low. Sweep water mode of testing was able to recover $98 \%$ of the feed nitrogen with a product purity of $90 \% \mathrm{~N}_{2}$. In both conventional permeation and vacuum modes of operation, a purified gas stream containing only about $5 \%$ $\mathrm{CO}_{2}$ was observed. The $\mathrm{N}_{2}$ recoveries were about $72 \%$ and $83 \%$, respectively. In the last three cases, pure water was utilized as a liquid membrane; thus feed gas humidification was avoided completely. Many experiments with a $40 \% \mathrm{CO}_{2}-$ $60 \% \mathrm{CH}_{4}$ feed mixture have provided similar performances.

Mathematical models have been deviloped for each mode of operation. The results obtained from these models describe the permeator behavior quite well. In addition, a generalized model has been developed which determines the effective liquid membrane thickness in a HFCLM permeator by solving the diffusion equation in two dimensions.

A purification loop was built for large-scale operation which provided feed gas and sweep gas to the permeator at any humidity between $0-100 \%$. Long-term experiments with pure water and $20 \mathrm{wt} \%$ DEA solution as membranes and either $40-60 \mathrm{CO}_{2}-\mathrm{N}_{2}$ or $\mathrm{CO}_{2}-\mathrm{CH}_{4}$ gas mixture as feed, demonstrated that the hollow-fiber-contained liquid membrane system can be used efficiently to separate gas mixtures with minimum maintenance over a prolonged period of time. In an industrial operation, a slow circulation of the liquid membrane with some arrangement for replenishing the liquid loss due to evaporation in the gas stream is desirable. It might eliminate the need for humidifying the gas streams completely. Moreover, the same arrangement might be used for in-situ replacement of the membrane if poisoned or contaminated by some unwanted constituents in the gas mixture.

The HFCLM technique has overcome the deficiencies of traditional liquid membrane systems by providing stable operation. Purification of biogas can be achieved to yield pipeline quality gas with high methane recovery. It is strongly recommended that the potential of this new technique be explored for 
other gas treating applications such as treatment of fuel gas available from biomass and/or coal gasification, removal of $\mathrm{CO}_{2}$ from a gas stream in denitrification bioreactor, removal of $\mathrm{SO}_{2} / \mathrm{NO} x$ from flue gas, natural gas processing, separation of higher olefins from hydrocarbons such as ethylene/ethane gas mixture, synthesis gas purification, oxygen enrichment of air, chlorine/hydrochloric acid gas separation from vent gases etc. Additionally, use of HFCLM modules as pre-treatment facilities for bulk purification before gas absorption towers for removing $\mathrm{H}_{2} \mathrm{~S}$ is expected to reduce the load and size of the towers drastically. 


\section{Section 1}

\section{INTRODUCTION}

Conventional gas purification technologies are not suitable for economic removal of carbon dioxide from methane obtained in biogas due to the small scale of production. Membrane-based separation techniques are suited for such processes. Liquid membranes are attractive in general since they possess high species permeability and selectivity. However, the traditional immobilized liquid membrane (ILM) technique suffers from the problems of membrane stability, membrane flooding and a costly configuration. A new liquid membrane technique which bypasses the shortcomings of ILM was developed under the joint sponsorship of NYSERDA, NYGAS and GRI since 1984. The new hollow-fibercontained liquid membrane (HFCLM) technique utilizes two sets of hydrophobic, microporous hollow fibers. The feed gas mixture is separated by preferential permeation of a gas species from one set of fibers to the other, through thin layers of liquids contained on the outside of the two sets of fibers. The fibers are packed together tightly in a shell. An account of this technique and its application to the purification of a landfill gas mixture using a sweep gas stream is available in NYSERDA Report 87-10. The current phase of the project was undertaken to investigate experimentally the short-term and long-term performances of the HFCLM permeation process for biogas purification using three different separation modes of sweep, vacuum and conventional permeation and development of mathematical models to predict the system performance under these modes of operation. The current phase of the project is also concerned with an experimental study of the continuous separation performance of a larger permeator containing 5000 ribers over periods one-month-long using the optimum mode of permeator operation. The overall task and task components are available in Table $1-1$.

In the first phase of the project, the separation of model biogas mixtures has been studied using four different permeation modes. The first mode uses a sweep gas through the permeate fibers to create the proper partial pressure driving force. The second mode uses a sweep liquid e.g., water to achieve the same end. The third mode explores liquid membrane operation as if the liquid membrane were a polymeric membrane in a conventional permeator. The fourth mode uses a 


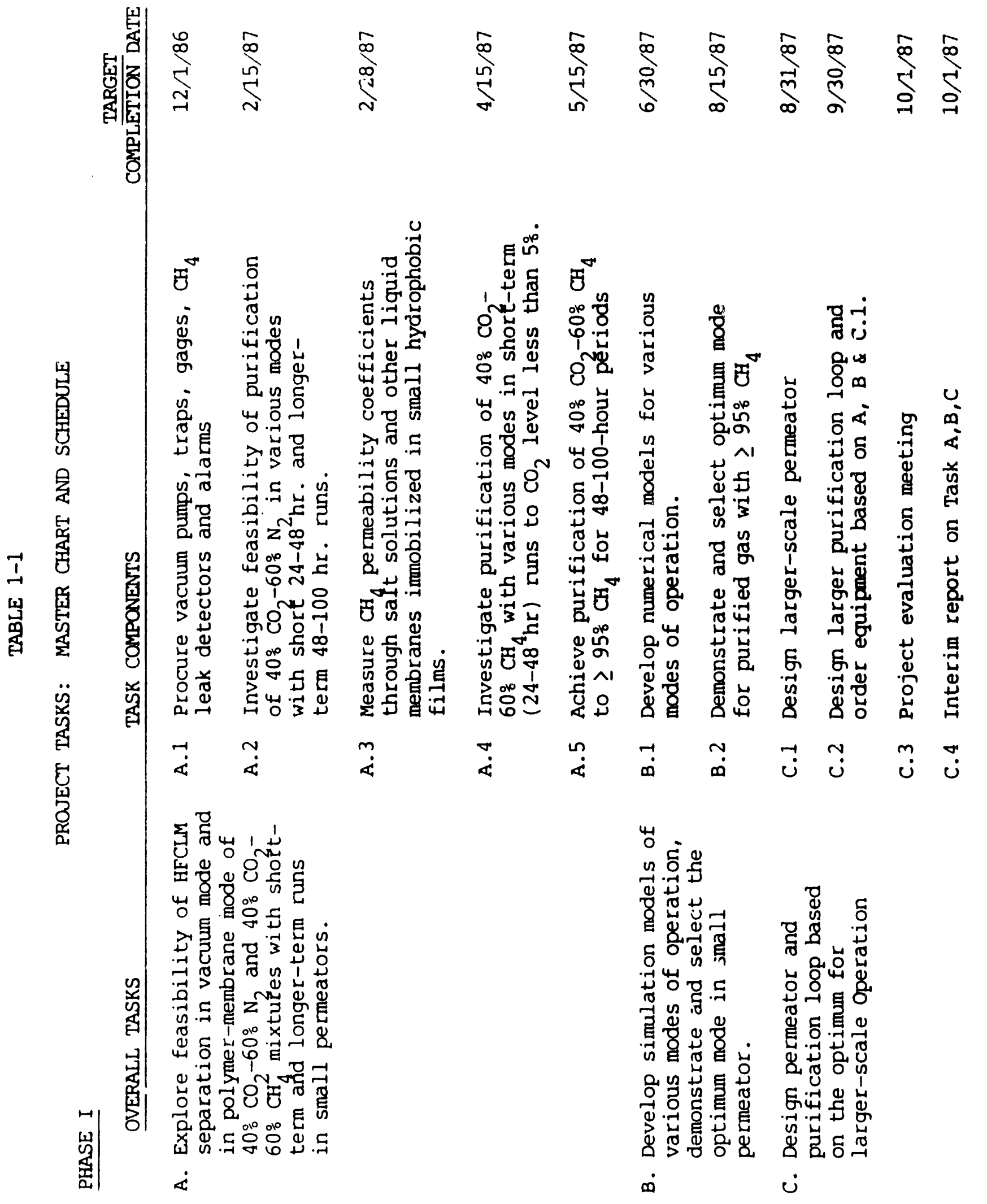




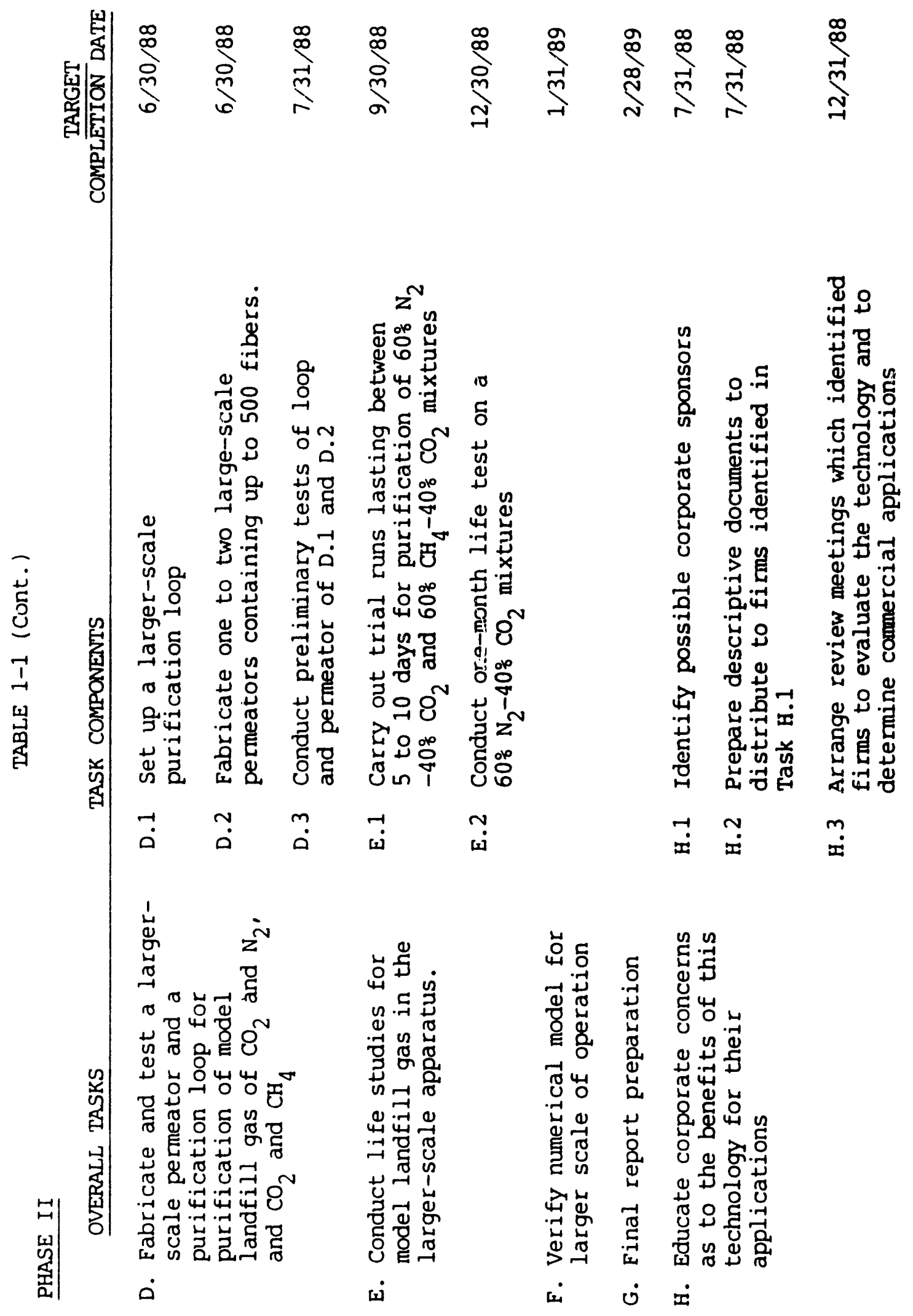


vacuum on the permeate side to create a partial pressure difference. The separation schemes are shown in Figure 1-1.

Experimental investigations of gas separation in small HFCLM permeators for short and extended separation runs in various modes were made mainly with a feed gas mixture consisting $40 \% \quad \mathrm{CO}_{2}$ and $60 \% \quad \mathrm{~N}_{2}$. For sweep gas mode of operation, helium was primarily used as a sweep gas. Quite a few experiments were carried out with $40 \% \mathrm{CO}_{2}-60 \% \mathrm{CH}_{4}$ gas mixture as feed and $\mathrm{N}_{2}$ as the sweep gas. Feed pressure was generally maintained at $45 \mathrm{psig.} \mathrm{The} \mathrm{liquid} \mathrm{membranes}$ investigated include water, aqueous $\mathrm{K}_{2} \mathrm{CO}_{3}$ solutions ( $7 \mathrm{wt} \%$ or $30 \mathrm{wt} \%$ ), aqueous solutions of 1-methyl-2-pyrrolidinone (NMP) (70 and 80 vol\%) and aqueous diethanolamine (DEA) solutions $(10,20$ and $30 \mathrm{wt} \%)$. Most experiments were done at $25^{\circ} \mathrm{C}$.

A number of hollow-fiber-contained liquid membrane permeators were developed specifically for the project. Sometimes these permeators were used in series configurations. Continuous experimental runs have been carried out on numerous occasions for periods as long as 100 hours to determine the operational stability of this new technique. The membrane liquid pressure was around 50 psig for 45 psig feed pressure.

The operational data of these and previously available permeators in the new modes have been compared with the numerical simulation results of mathematical models developed for each mode. Species permeability information was experimentally developed separately through a few membranes to this end. Using the observed separation results and numerical simulations, a preliminary economic comparison of the various modes of operation of HFCLM permeators has been carried out.

In the second part of the project, a larger-scale purification loop was designed and constructed. Using numerical models, a permeator was also designed for larger-scale operation. Three large permeators, each containing 7000 microporous polypropylene hollow fibers, were fabricated for the project. Longterm purification runs were carried out with both $\mathrm{CO}_{2}-\mathrm{N}_{2}$ and $\mathrm{CO}_{2}-\mathrm{CH}_{4}$ feed mixtures for periods as long as 240 hours. The liquid membranes utilized were pure water and aqueous 20 wt $\mathrm{DEA}$ solution, respectively. A one-month-long 

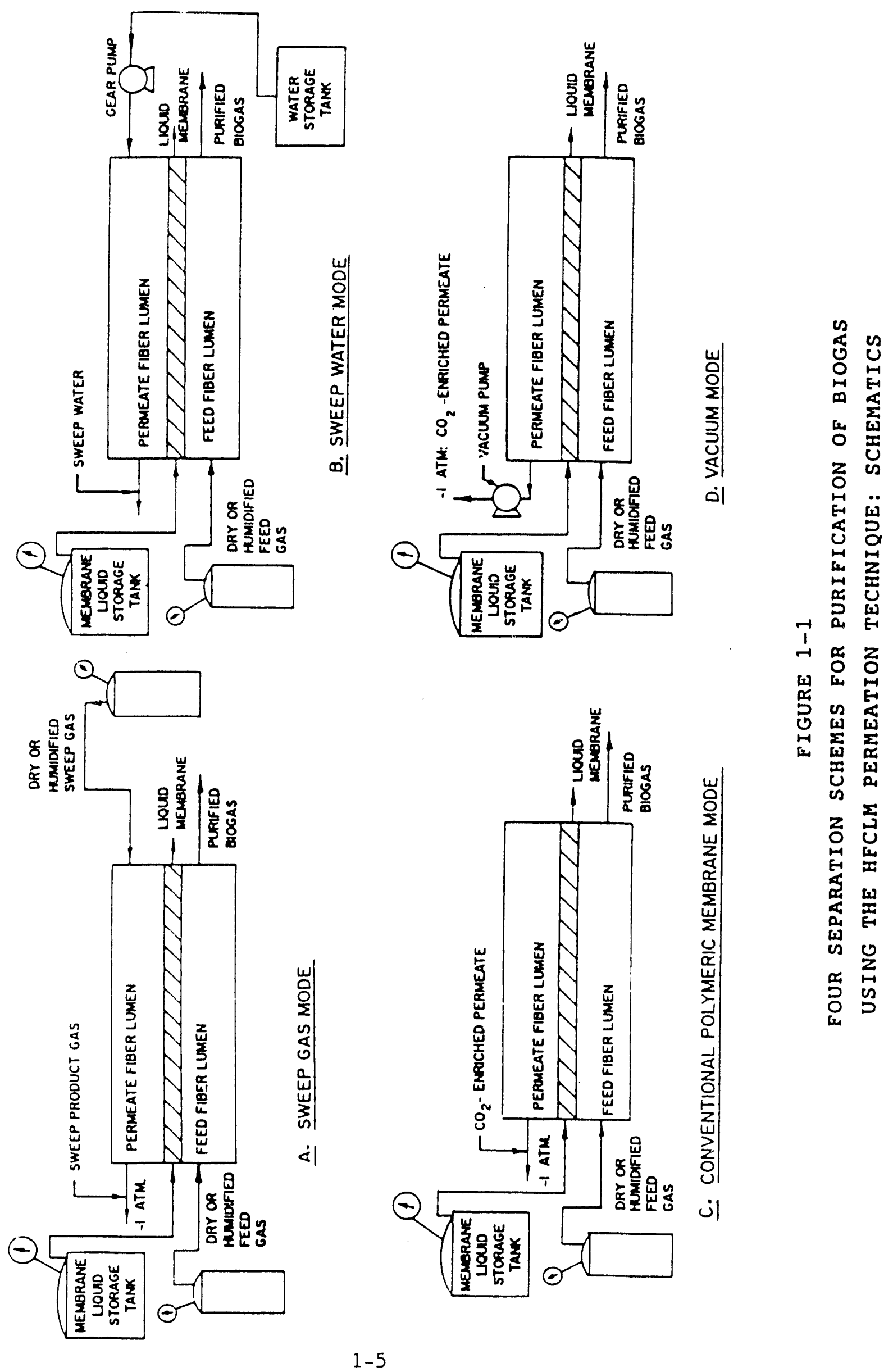
life test was also conducted using a feed gas mixture containing $\mathrm{CO}_{2}$ and $\mathrm{N}_{2}$ and an aqueous $20 \mathrm{wt} \%$ DEA solution as a liquid membrane. The membrane thickness in each large permeator was determined utilizing the mathematical models developed earlier. 


\section{Section 2}

\section{MODELING AND SIMULATION}

The modeling aspects of gas mixture separation in hollow-fiber-contained liquid membrane (HFCLM) permeators under various modes of operation are considered in this section. The following assumptions are used to analyze the gas permeation and separation of a binary feed gas mixture in a HFCLM permeator under various modes of operation:

1. The permeability coefficient of each nonreacting gas component through water as well as aqueous solution of DEA is same as that of the pure gas, and is independent of gas pressure.

2. There exists an effective liquid membrane thickness, d, along the permeator length as well as at all radial locations in the fiber bundle.

3. There is no mass transfer resistance in the gas phases.

4. There is negligible diffusion along the mean gas flow path compared to the bulk gas flow.

5. Plug flow model can be used for both gas streams.

6. Pressure drops through fiber lumen are governed by the Hagen-Poiseuille equation.

7. The viscosity of pure gas or gas mixture is independent of pressure but the gas mixture viscosities are dependent on mixture composition.

8. Axial diffusion in the stationary membrane liquid is insignificant compared to the radial diffusion.

9. End effects inside the permeator are negligible.

10. There is negligible deformation of the hollow fibers under external pressure.

11. Separation or pressure drop is unaffected by the small movement of the membrane liquid (due to replenishment of membrane liquid lost by evaporation and/or leakage through defective fibers).

Permeability coefficient of a reactive species, for example, $\mathrm{CO}_{2}$, through aqueous solution DEA is strongly dependent on the partial pressure of the gas species at the feed and sweep (or permeate) sides. A detailed analysis is given in a latter part of this section. 


\section{ANALYSIS OF GAS MIXTURE SEPARATION IN A HFCLM PERMEATOR}

\section{SWEEP GAS MODE OP OPERATION}

The mathematical analysis of model biogas mixture separation with a sweep gas in a HFCLM permeator having feed and sweep fibers of different dimensions is considered here. A schematic of the hollow-fiber-contained liquid membrane permeator under sweep gas mode of operation is presented in Figure 2-1. Only one feed fiber and one sweep fiber of different dimensions are shown for simplicity. Different numbers of each kind of fiber were needed to maintain the same permeation area in feed side and sweep side. The sweep gas stream flows countercurrent to the feed gas stream. A binary mixture of $\mathrm{CO}_{2}$ and $\mathrm{N}_{2}$ ( or $\mathrm{CH}_{4}$ ) is used as feed whereas pure helium (or $\mathrm{N}_{2}$ ) is used as sweep.

An overall material balance between the feed inlet end and any location at a distance ' $l$ ' from the sweep inlet end leads to

$$
N_{F} L-N_{S} V=N_{F} L_{f}-N_{S} V_{f}
$$

Here $L$ and $V$ are local molar feed and sweep flow rates per fiber; $N_{F}$ and $N_{S}$ are total number of feed and sweep fibers, respectively. The component balances can be written as

$\mathrm{CO}_{2}: \quad \mathrm{N}_{F} \mathrm{Lx}_{\mathrm{X}}-\mathrm{N}_{\mathrm{S}} \mathrm{Vy}_{\mathrm{H}}=\mathrm{N}_{\mathrm{F}} \mathrm{L}_{\mathrm{f}} \mathrm{x}_{\mathrm{f}}-\mathrm{N}_{\mathrm{S}} \mathrm{V}_{\mathrm{f}} \mathrm{y}_{\mathrm{f}}$

$\mathrm{N}_{2}\left(\right.$ or $\left.\mathrm{CH}_{4}\right): \quad \mathrm{N}_{\mathrm{F}} \mathrm{L}(1-\mathrm{x}-\mathrm{u})-\mathrm{N}_{\mathrm{S}} \mathrm{V}(1-\mathrm{y}-\mathrm{v})=\mathrm{N}_{\mathrm{F}} \mathrm{L}_{\mathrm{f}}\left(1-\mathrm{x}_{\mathrm{f}}-\mathrm{u}_{\mathrm{f}}\right)-\mathrm{N}_{\mathrm{S}} \mathrm{V}_{\mathrm{f}}\left(1-\mathrm{y}_{\mathrm{f}}-\mathrm{v}_{\mathrm{f}}\right)$

He $\left(\right.$ or $\left.N_{2}\right): \quad N_{F} L u-N_{S} V V=N_{F} L_{f} u_{f}-N_{S} V_{f} V_{f}$

For equal permeation areas in feed and sweep sides, one can write

$$
N_{F} \pi D_{F O} l_{t}=N_{S} \pi D_{S O} l_{t}
$$

Therefore

$$
\mathrm{N}_{\mathrm{F}} / \mathrm{N}_{\mathrm{S}}=\mathrm{D}_{\mathrm{SO}} / \mathrm{D}_{\mathrm{FO}}=\theta(\text { say })
$$




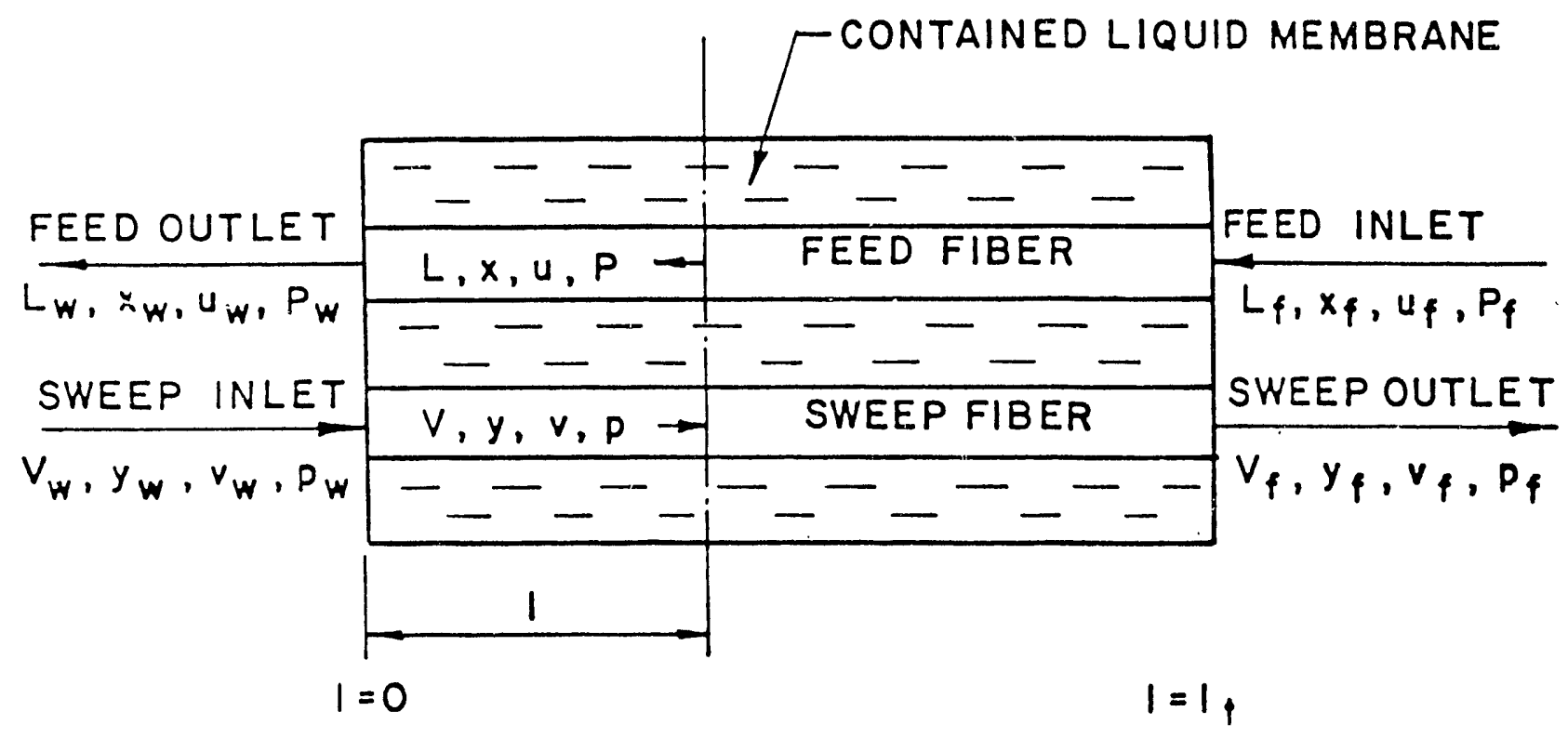

FIGURE $\quad 2-1$

SCHEMATIC OF A HFCLM PERMEATOR FOR MODELING SWEEP GAS MODE OF OPERATION 
If the axial coordinate ' 1 ' is positive in the direction of sweep gas flow, the governing differential equations for permeation for three species are given by

$\left.\mathrm{CO}_{2}: \quad \mathrm{d}(\mathrm{Lx}) / \mathrm{d}\right]=\pi \mathrm{D}_{\mathrm{FO}}\left(\mathrm{Q}_{\mathrm{a}} / \mathrm{d}\right)[\mathrm{Px}-\mathrm{py}]$

$\mathrm{N}_{2}\left(\right.$ or $\left.\left.\mathrm{CH}_{4}\right): \quad d[L(1-x-u)] / d\right]=\pi D_{F O}\left(Q_{b} / d\right)[P(1-x-u)-p(1-y-v)]$

He $\left(\right.$ or $\left.N_{2}\right): \quad d(L u) / d l=\pi D_{F 0}\left(Q_{c} / d\right)[P u-p v]$

The differential equations governing the pressure drop in the two gas streams in the two sets of fiber lumina are :

Feed side : $\quad d P / d]=128 R T L \mu_{F} /\left[\begin{array}{lll}\pi & D_{F I}^{4}\end{array}\right]$

Sweep side : $d p / d I=-128 R T V \mu_{S} /\left[\begin{array}{lll}\pi & D_{S I}^{4}\end{array}\right]$

Eqs. (2-7) through (2-11) may be converted to dimensionless forms as follows:

$$
\begin{aligned}
& d\left(L^{\star} x\right) / d S=\alpha_{a}\left[r_{1} x-r_{2} y\right] \\
& d\left[L^{\star}(1-x-u)\right] / d S=\alpha_{b}\left[r_{1}(1-x-u)-r_{2}(1-y-v)\right] \\
& d\left(L^{\star} u\right) / d S=\alpha_{c}\left[r_{1} u-r_{2} v\right] \\
& d r_{1} / d S=\beta \mu_{F}^{\star} L^{\star} / r_{1} \\
& d r_{2} / d S=-\beta\left(D_{F I} / D_{S I}\right)^{4} \mu_{S}^{\star} v^{\star} / r_{2}
\end{aligned}
$$

where dimensionless area,

$$
S=\pi D_{F O}\left(Q_{\text {ref }} / d\right)\left(P_{\text {ref }} / L_{\text {ref }}\right) l
$$

dimensionless pressures,

$$
r_{1}=P / P_{\text {ref }} ; r_{2}=p / P_{\text {ref }}
$$


dimensionless flow rates,

$L^{\star}=L / L_{\text {ref }} ; V^{\star}=V / L_{\text {ref }}$

dimensionless viscosities,

$\mu_{F}^{*}=\mu_{F} / \mu_{\text {ref }} ; \mu_{S}^{*}=\mu_{S} / \mu_{\text {ref }}$

$\alpha_{a}=Q_{a} / Q_{\text {ref }} ; \alpha_{b}=Q_{b} / Q_{\text {ref }} ; \alpha_{c}=Q_{c} / Q_{\text {ref }}$

and

$B=128 R T L_{\text {ref }}^{2} \mu_{r e f} /\left[\pi^{2} D_{F I}^{4} D_{F O}\left(Q_{r e f} / d\right) P_{r e f}^{3}\right]$

Adding Eqs. $(2-12),(2-13)$ and $(2-14)$, one gets

$\mathrm{dL}^{*} / \mathrm{dS}=\alpha_{\mathrm{a}}\left[\gamma_{1} x-r_{2} y\right]+\alpha_{b}\left[r_{1}(1-x-u)-r_{2}(1-y-v)\right]+\alpha_{c}\left[r_{1} u-r_{2} v\right]$

From Eqs. $(2-1)$ and $(2-2)$

$N_{F} d L=N_{S} d V ; N_{F} d(L x)=N_{S} d(V y)$

which leads to

$d V^{\star} / d S=\theta d L^{*} / d S ; d\left(V^{\star} y\right) / d S=\theta d\left(L^{\star} x\right) / d S$

Therefore,

$d V^{\star} / d S=\theta\left[\alpha_{a}\left\{r_{1} x-r_{2} y\right\}+\alpha_{b}\left\{r_{1}(1-x-u)-r_{2}(1-y-v)\right\}+\alpha_{c}\left\{r_{1} u-r_{2} v\right]\right]$

Now, it should be noted that

$L^{*} d x / d S=d\left(L^{*} x\right) / d S-x d L^{*} / d S$

$V^{\star} d y / d S=d\left(V^{*} y\right) / d S-y d V^{*} / d S$

Combining these mathematical relations and Eqs. (2-12), (2-23) and (2-24), one can get 
$d x / d S=\left\{\alpha_{a}(1-x)\left(r_{1} x-r_{2} y\right)-\alpha_{b} x\left\{r_{1}(1-x-u)-r_{2}(1-y-v)\right\}-\alpha_{c} x\left(r_{1} u-r_{2} v\right)\right] / L^{\star}$

and

$d y / d S=\theta\left[\alpha_{a}(1-y)\left(r_{1} x-r_{2} y\right)-\alpha_{b} y\left\{r_{1}(1-x-u)-r_{2}(1-y-v)\right\}-\alpha_{c} y\left(r_{1} u-r_{2} v\right)\right] / v^{*}$

Following a similar approach, one can also derive,

$d u / d S=\left\{-\alpha_{a} u\left(r_{1} x-r_{2} y\right)-\alpha_{b} u\left\{r_{1}(1-x-u)-r_{2}(1-y-v)\right\}+\alpha_{c}(1-u)\left(\gamma_{1} u-r_{2} v\right)\right] / L^{\star}(2-27)$

$d v / d S=\theta\left[-\alpha_{a} v\left(\gamma_{1} x-r_{2} y\right)-\alpha_{b} v\left\{\gamma_{1}(1-x-u)-\gamma_{2}(1-y-v)\right\}+\alpha_{c}(1-v)\left(\gamma_{1} u-r_{2} v\right)\right] / v^{\star}(2-28)$

Equations (2-23) through (2-28), (2-15) and (2-16) constitute a set of eight nonlinear coupled ordinary differential equations in $V^{*}, L^{*}, y, x, v, u, r_{1}$ and $r_{2}$ which have to be solved simultaneously using the proper boundary conditions at $1=0$ and $1=l_{t}$. Generally, it is convenient to specify the domain of the independent variable between 0 and 1 . In this case, the total dimensionless area $S_{t}$ (for $l=l_{t}$ ) can be made equal to unity with proper choice of reference parameters (i.e., $Q_{\text {ref, }} P_{\text {ref }}$ and $L_{\text {ref }}$ ) in definition (2-17) (Majumdar, 1986). The boundary conditions for the above set of equations can then be written as

$S=0 \Rightarrow V^{\star}=V_{w}^{\star} ; y=0\left(=y_{w}\right) ; v=1\left(=v_{w}\right)$

$S=1 \Rightarrow L^{\star}=L_{f}^{\star} ; x=x_{f} ; u=0\left(=u_{f}\right) ; r_{1}=r_{1 f} ; r_{2}=r_{2 f}$

The other unknown boundary values such as $L_{w}^{*}, x_{w}, u_{w}, r_{1 w}, r_{2 w}($ at $S=0)$ and $v_{f}^{\star}, y_{f}, v_{f}$ (at $S=1$ ) were obtained by solving the system of differential equations numerically.

The gas mixture viscosities used in the above and subsequent analyses were computed according to the following equation (Reid et al., 1977):

$\bar{\mu}=\sum_{i}\left\lfloor z_{i} \mu_{i} / \sum_{j} \phi_{i j} z_{j}\right\rfloor$

where the constants $\phi_{i j}-$ are obtained as (Wilke, 1950) :

$\phi_{i j}=\left[1+\left(\mu_{i} / \mu_{j}\right)^{1 / 2}\left(M_{w j} / M_{w i}\right)^{1 / 4}\right]^{2} /\left[8\left(1+M_{w i} / M_{w j}\right)\right]^{1 / 2}$ 
Here $z_{i}, \mu_{i}, M_{w i}$ are the mole fraction, viscosity and the molecular weight of the $i$ th species, respectively.

\section{CONVENTIONAL OR VACUUM MODE OP OPERATION}

Figure 2-2 shows the schematic of vacuum or conventional mode of membrane gas separation with countercurrent flow. The governing equations for these two modes of operation are identical. Note that these two modes do not utilizis any sweep gas; therefore the feed or the permeate gas stream is considered to be a mixture of two components only. The governing differential equations in dimensionless form are easily obtained by putting $u=0$ and $v=0$ [i.e., no helium (or nitrogen in the case of $\mathrm{CH}_{4}$ in feed) in feed and permeate stream) in Eqs. $(2-23)$ through $(2-26),(2-15)$ and $(2-16)$. The new reduced equations are

$\mathrm{dL}^{*} / \mathrm{dS}=\alpha_{\mathrm{a}}\left[r_{1} \mathrm{x}-\gamma_{2} \mathrm{y}\right]+\alpha_{\mathrm{b}}\left[\gamma_{1}(1-\mathrm{x})-r_{2}(1-\mathrm{y})\right]$

$d V^{*} / d S=\theta\left[\alpha_{a}\left(r_{i} x-r_{2} y\right\}+\alpha_{b}\left\{r_{1}(1-x)-r_{2}(1-y)\right\}\right]$

$\left.\mathrm{dy} / \mathrm{dS}=\theta \mid \alpha_{a}(1-\mathrm{y})\left(r_{1} x-r_{2} y\right)-\alpha_{b} y\left[r_{1}(1-x)-r_{2}(1-y)\right\}\right] / V^{\star}$

$d x / d S=\left[\alpha_{a}(1-x)\left(\gamma_{1} x-\gamma_{2} y\right)-\alpha_{b} x\left\{\gamma_{1}(1-x)-r_{2}(1-y)\right\}\right] / L^{*}$

$d r_{1} / d S=B \mu_{F}^{\star} L^{\star} / r_{1}$

$d r_{2} / d S=-\beta\left(D_{F I} / D_{S I}\right)^{4} \mu_{P}^{\star} v^{\star} / r_{2}$

In Eq. (2-38), subscript ' $\mathrm{P}$ ' is used to identify the permeate stream. The boundary conditions for the above set of equations are :

at $S=0 \Rightarrow V^{\star}=0\left(=V_{w}^{\star}\right) ; y=y_{w}=y\left(r_{1}, r_{2}, x\right)$

and

at $S=1 \Rightarrow L^{\star}=L_{f}^{*} ; x=x_{f} ; r_{1}=r_{1 f} ; r_{2}=r_{2 f}$

Since $y_{w}$ can not be specified explicitly, it has to be determined from other equations . 


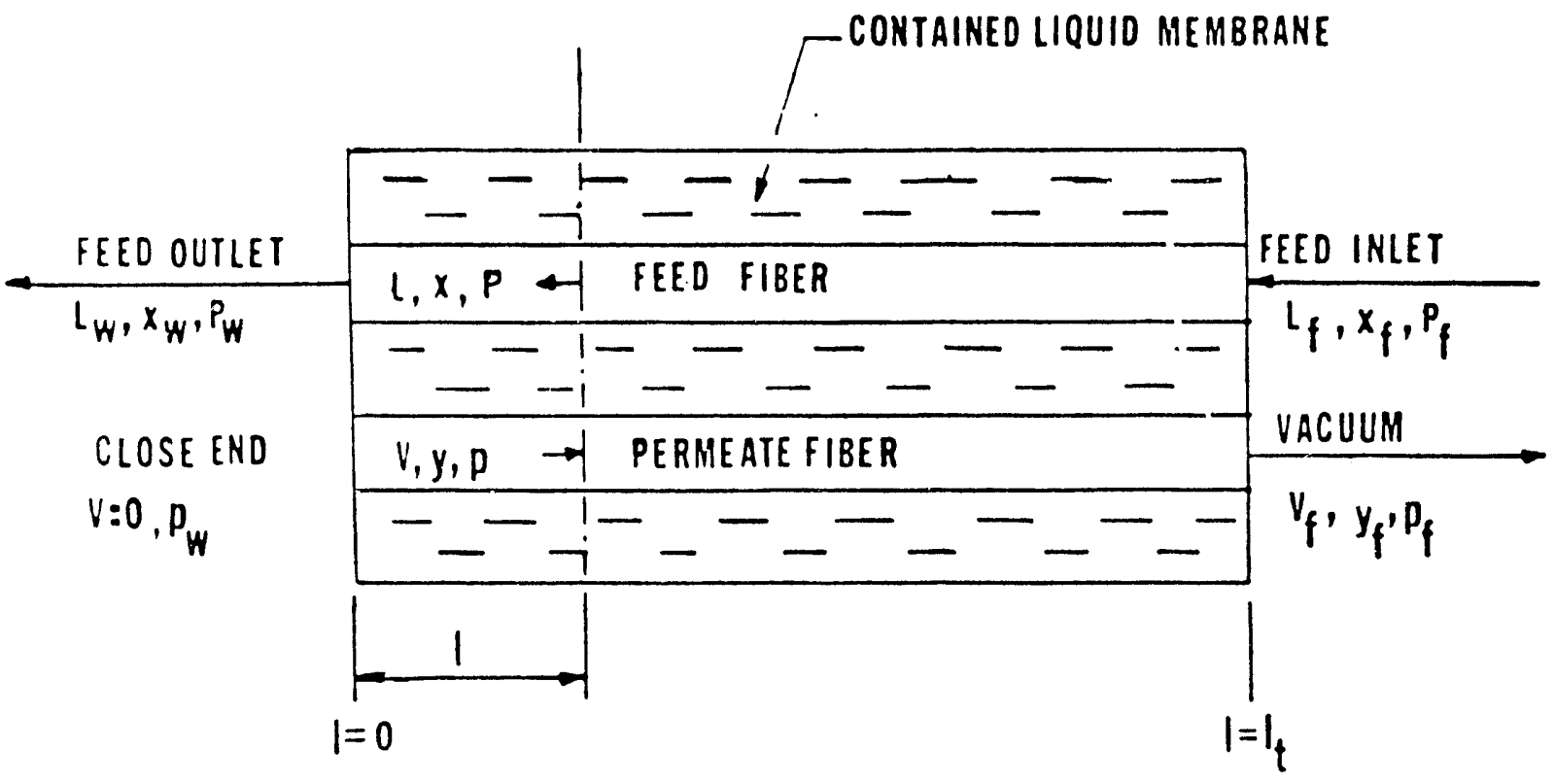

FIGURE 2-2

SCHEMATIC OF A HFCLM PERMEATOR FOR MODELING VACUUM OR CONVENTIONAL MODE OF OPERATION 
We can write the permeation equations for two components as follows:

$$
\begin{aligned}
& d\left(V^{\star} y\right) / d S=\theta \alpha_{a}\left\{\gamma_{1} x-r_{2} y\right) \\
& d\left[V^{\star}(1-y)\right] / d S=\theta \alpha_{b}\left[\gamma_{1}(1-x)-r_{2}(1-y)\right\}
\end{aligned}
$$

Dividing Eq. $(2-41)$ by Eq. (2-42) yields

$$
\begin{aligned}
\left(V^{\star} d y+y d V^{\star}\right) /\left[V^{\star} d(1-y)+\right. & \left.(1-y) d V^{\star}\right] \\
= & {\left[\alpha_{a}\left\{\gamma_{1} x-r_{2} y\right\}\right] /\left[\alpha_{b}\left[\gamma_{1}(1-x)-r_{2}(1-y)\right]\right] }
\end{aligned}
$$

Applying Eq. $(2-43)$ at $S=0$ where $V^{\star}=0$, we get

$$
y_{w} /\left(1-y_{w}\right)=\left[\alpha_{a}\left\{r_{1 w} x_{w}-r_{2 w} y_{w}\right\}\right] /\left[\alpha_{b}\left[r_{1 w}\left(1-x_{w}\right)-r_{2 w}\left(1-y_{w}\right)\right\}\right]
$$

Thus $y_{w}$ is determined by solving the above quadratic equation as follows

$$
y_{w}=\frac{\begin{array}{l}
\left\{\alpha_{b} \gamma_{1 w}+\left(\alpha_{a}-\alpha_{b}\right)\left(\gamma_{1 w} x_{w}+\gamma_{2 w}\right)\right\} \\
-\left[\left\{\alpha_{b} \gamma_{1 w}+\left(\alpha_{a}-\alpha_{b}\right)\left(\gamma_{1 w} x_{w}+\gamma_{2 w}\right)\right\}^{2}-4 \alpha_{a} \gamma_{1 w} \gamma_{2 w} x_{w}\left(\alpha_{a}-\alpha_{b}\right)\right\}^{1 / 2}
\end{array}}{2 \gamma_{2 w}\left(\alpha_{a}-\alpha_{b}\right)}
$$

It should be noted that Eq. $(2-35)$ is indeterminate at $S=0$. Therefore, L'Hospital rule is applied to determine the derivative at that point by differentiating the numerator and denominator of Eq. (2-35) with respect to ' $S$ '. The equation. is given by

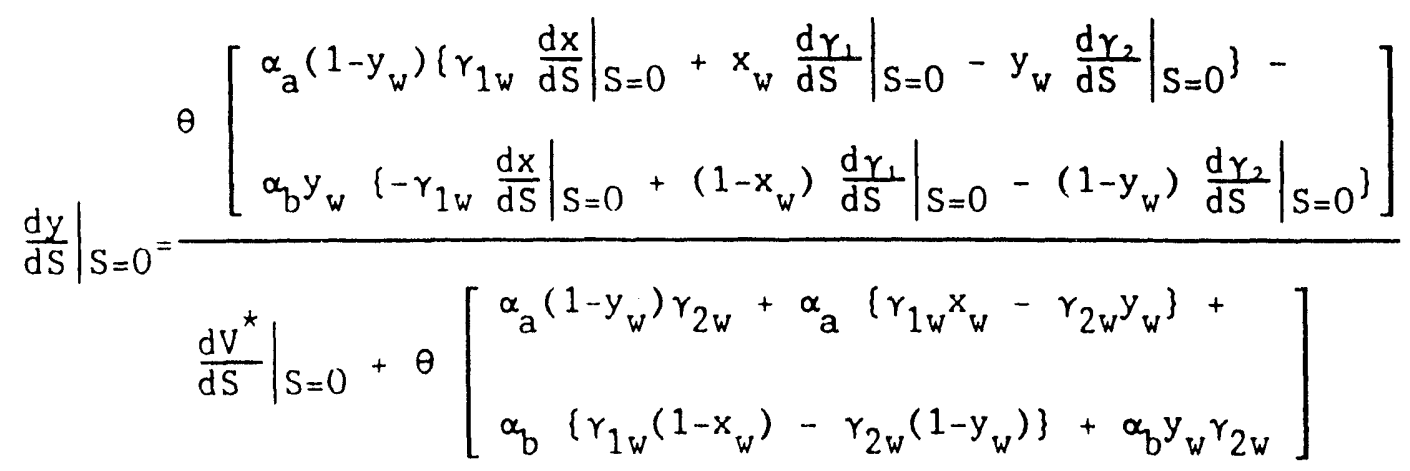


Since $V^{\star}=0$ at $S=0$, therefore, $\left.\frac{d Y_{2}}{d S}\right|_{S=0}=0$ and substituting $\left.\frac{d V^{\star}}{d S}\right|_{S=0}$ from $\mathrm{Eq} \cdot(2-34)$ at $\mathrm{S}=0$ in Eq. $(2-46)$ one can get

$$
\left.\frac{d y}{d S}\right|_{S=0}=\frac{\left[\begin{array}{l}
\left.\frac{d x}{d S}\right|_{S=0}\left(\alpha_{a}\left(1-y_{w}\right) r_{1 w}+\alpha_{b} y_{w} r_{1 w}\right\}+ \\
\left.\frac{d \gamma_{1}}{d S}\right|_{S=0}\left(\alpha_{a}\left(1-y_{w}\right) x_{w}-\alpha_{b}\left(1-x_{w}\right) y_{w}\right\}
\end{array}\right]}{\left[\begin{array}{l}
2\left(\alpha_{a}-\alpha_{b}\right) r_{1 w} x_{w}+\alpha_{a} r_{2 w} \\
-3\left(\alpha_{a}-\alpha_{b}\right) r_{2 w} y_{w}+2\left\{\alpha_{b}\left(r_{1 w}-r_{2 w}\right)\right\}
\end{array}\right]}
$$

\section{SWEEP HATER MODE OP OPERATION}

The schematic for this particular configuration is shown in Figure 2-3. Here, fresh water is introduced countercurrent to the feed gas mixture through the bore of sweep fibers. In this mode, permeating gases first desorb from the stationary liquid membrane at the permeate side, then diffuse through the gas phase confined in the pores of the microporols wall of the hollow fiber and are finally absorbed in the flowing sweep water. For modeling a HFCLM permeator under sweep water mode of operation, the following additional assumptions, apart from those considered earlier, are necessary:

1. There is no gas phase mass transfer resistance in the pores of microporous fiber wall on the sweep water side.

2. The gas pressure $\mathrm{P}_{i}$ and gas composition $y_{i}$ inside the pores of the microporous fiber wall of sweep water side controls the permeation process; the sweep water pressure has no effect.

The governing differential equations for permeation for the two species are given by

$$
\begin{array}{cc}
C_{2}: & d(L x) / d l=\pi D_{F O}\left(Q_{a} / d\right)\left\lfloor P x-p_{i} y_{i}\right] \\
N_{2}\left(\operatorname{or~}\left(H_{4}\right):\right. & d\left[L(1-x) \mid / d l=\pi D_{F O}\left(Q_{b} / d\right)\left[P(1-x)-p_{i}\left(1-y_{i}\right)\right]\right.
\end{array}
$$




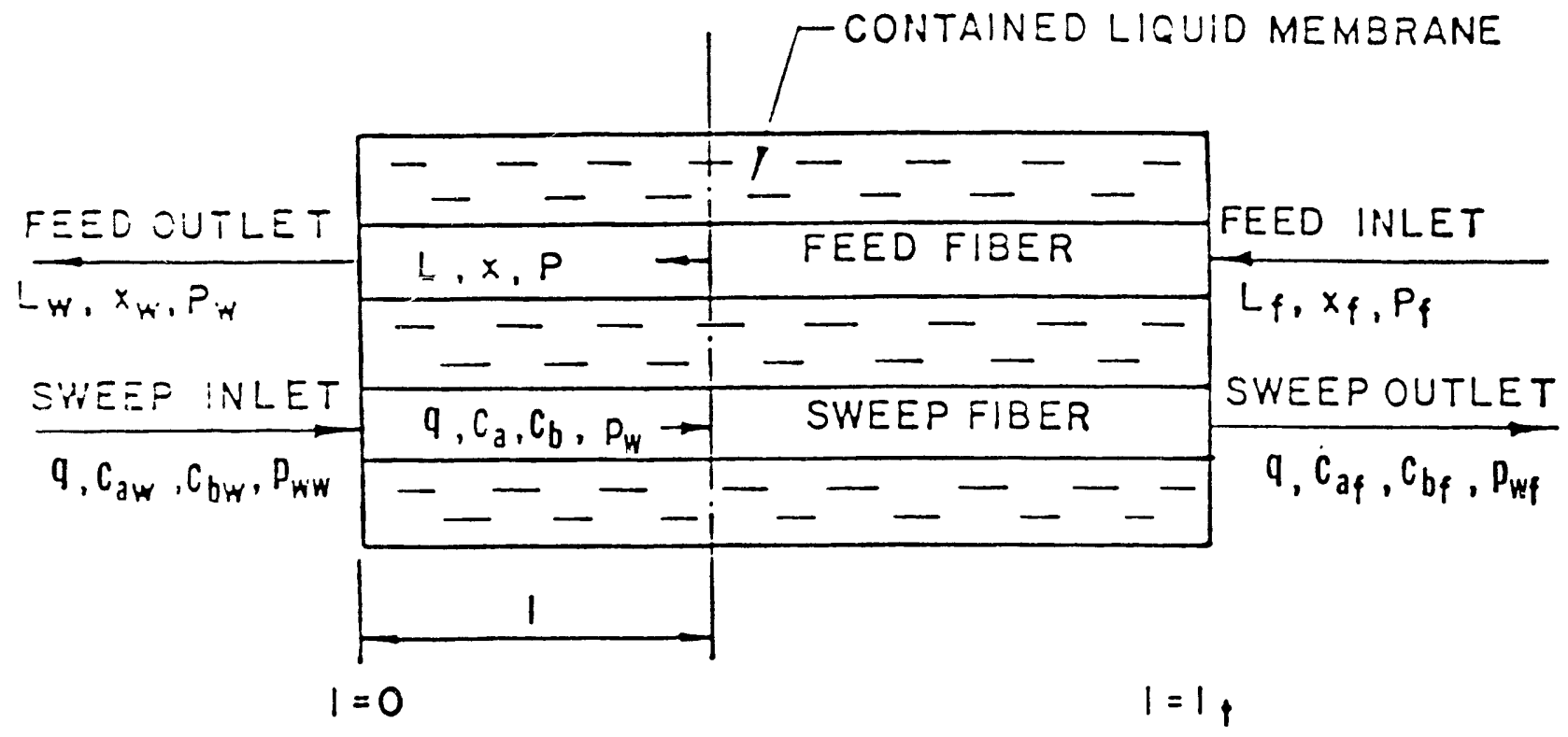

FIGURE 2-3

SCHEMATIC OF A HFCLM PERMEATOR FOR MODELING

SWEEP WATER MODE OF OPERATION 
The water side governing equations for the two species with $q$, the water flow rate/fiber, can be written as

$$
\begin{aligned}
& \mathrm{CO}_{2}: \quad \mathrm{qdC} / \mathrm{dl}=\left(\mathrm{k}_{\mathrm{m}}\right)_{\mathrm{a}}\left[\mathrm{S}_{\mathrm{a}} \mathrm{p}_{\mathrm{i}} \mathrm{y}_{\mathrm{i}}-\mathrm{C}_{\mathrm{a}}\right] \pi \mathrm{D}_{\mathrm{SI}} \\
& \mathrm{N}_{2}\left(\text { or } \mathrm{CH}_{4}\right): \quad \mathrm{q} d \mathrm{dC}_{b} / \mathrm{dl}=\left(k_{m}\right)_{b}\left[\mathrm{~S}_{\mathrm{b}} \mathrm{p}_{\mathrm{i}}\left(1-\mathrm{y}_{\mathrm{i}}\right)-\mathrm{C}_{\mathrm{b}}\right] \pi \mathrm{D}_{\mathrm{SI}}
\end{aligned}
$$

where $k_{m}$ is obtained either from the local value of Sherwood number based on local concentration difference, or the Sherwood number based on the arithmetic-mean concentration difference (Skeliand, 1974)

$\left(\mathrm{N}_{\mathrm{Sh}_{i}}\right)_{1}=\left\{\left.\left(\mathrm{k}_{\mathrm{m}}\right)_{i}\right|_{1} \mathrm{D}_{\mathrm{SI}} / \mathrm{D}_{\mathrm{i}}\right\}=\mathrm{f}_{1 \mathrm{i}}$

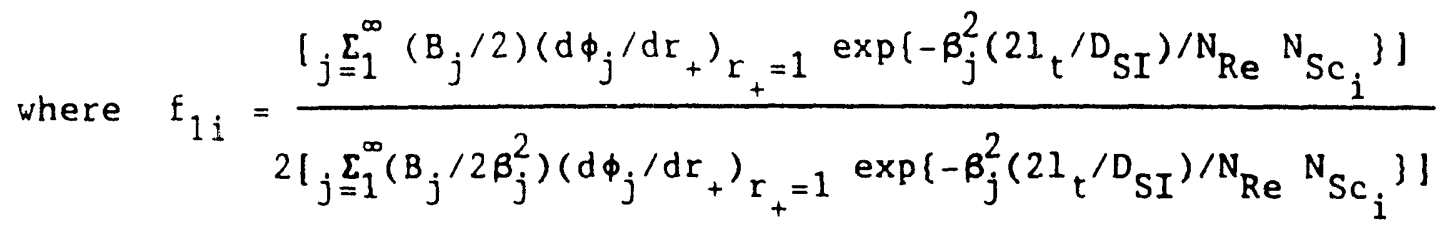

and $\left.\left(\mathrm{N}_{\mathrm{Sh}}\right)_{\mathrm{i}}\right)_{\mathrm{avg}}=\left\{\left.\left(\mathrm{k}_{\mathrm{m}}\right)_{\mathrm{i}}\right|_{\text {avg }} \mathrm{D}_{\mathrm{SI}} / \mathrm{D}_{\mathrm{i}}\right\}=0.5\left(\mathrm{D}_{\mathrm{SI}} / \mathrm{I}_{\mathrm{t}}\right) \mathrm{N}_{\mathrm{Re}} \mathrm{N}_{\mathrm{Sc}_{\mathrm{i}}} \mathrm{f}_{2 \mathrm{i}}$

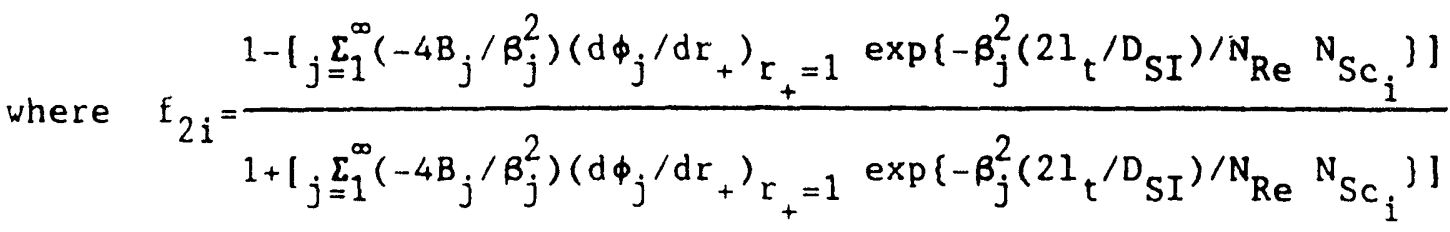

where $\quad \beta_{j}=4(j-1)+(8 / 3) ; j=1,2,3, \cdots$

and

$$
\left(-B_{j} / 2\right)\left(d \phi_{j} / d r_{+}\right)_{r_{+}}=1=1.01276 B_{j}^{-1 / 3}
$$

The differential equations governing the pressure drops through the two different fibser sets are:

Feed side : $\quad d P / d l=128 R T L \mu_{F} /\left[\begin{array}{lll}\pi & P & D_{F I}^{4}\end{array}\right]$

Sweep side : $d p_{w} / d l=-128 q \mu_{w} /\left[\pi D_{S I}^{4}\right]$

Equations $(2-48)$ to $(2-51),(2-58)$ and $(2-59)$ are converted to dimensionless forms as follows : 


$$
\begin{aligned}
& d\left(L^{\star} x\right) / d S=\alpha\left[r_{1} x-r_{i} y_{i}\right] \\
& d\left[L^{\star}(1-x)\right] / d S=\left[r_{1}(1-x)-r_{i}\left(1-y_{i}\right)\right] \\
& d C_{a}^{\star} / d S=R_{a}\left[s r_{i} y_{i}-C_{a}^{*}\right] \\
& d C_{b}^{\star} / d S=R_{b}\left[r_{i}\left(1-y_{i}\right)-C_{b}^{*}\right] \\
& d r_{1} / d S=\beta_{F} \mu_{F}^{\star} L^{*} / r_{1} \\
& d r_{w} / d S=-\beta_{S}
\end{aligned}
$$

where

$$
\begin{aligned}
& r_{1}=P_{f} / P_{\text {ref }} ; r_{w}=P_{w} / P_{\text {ref }} ; r_{i}=p_{i} / P_{\text {ref }} \\
& C_{a}^{*}=C_{a} /\left(S_{b} P_{r e f}\right) ; C_{b}^{*}=C_{b} /\left(S_{b} P_{r e f}\right) \\
& \alpha=Q_{a} / Q_{b} ; s=S_{a} / S_{b} \\
& \beta_{F}=128 R T L_{r e f}^{2} \mu_{r e f} /\left\{\pi^{2} D_{F I}^{4} D_{F O}\left(Q_{b} / d\right) P_{r e f}^{3}\right\} \\
& \beta_{S}=128 \mu_{w} q L_{r e f} /\left\{\pi^{2} D_{S I}^{4} D_{F O}\left(Q_{b} / d\right) P_{r e f}^{2}\right\} \\
& R_{a}=\left\{\left(k_{m}\right)_{a} D_{S I} L_{r e f}\right\} /\left\{\left(Q_{b} / d\right) D_{F O} q P_{r e f}\right\} \\
& \text { and } \\
& R_{b}=\left\{\left(k_{m}\right)_{b} D_{S I} L_{r e f}\right\} /\left\{\left(Q_{b} / d\right) D_{F 0} q P_{r e f}\right\}
\end{aligned}
$$

From Eqs. $(2-60)$ and $(2-61)$ one can easily get

$$
\begin{aligned}
& \mathrm{dL}^{\star} / \mathrm{dS}=\alpha\left[\gamma_{1} x-\gamma_{i} y_{i}\right]+\left[\gamma_{1}(1-x)-r_{i}\left(1-y_{i}\right)\right] \\
& \text { and } \\
& d x / d S=\left[\alpha(1-x)\left(\gamma_{1} x-r_{i} y_{i}\right)-x\left\{r_{1}(1-x)-r_{i}\left(1-y_{i}\right)\right\}\right] / L^{\star}
\end{aligned}
$$

From $\mathrm{CO}_{2}$ balance, we can write 
$N_{F} d(L x) / d l=N_{S} q d C_{a} / d l$

Similarly, from $\mathrm{N}_{2}$ (or $\mathrm{CH}_{4}$ ) balance

$N_{F} \cdot d[L(1-x)] / d l=N_{S} q d C_{b} / d l$

Equations $(2-75)$ and $(2-76)$ along with Eqs. $(2-60)$ to $(2-63)$ lead to

$r_{1} x-r_{i} y_{i}=\psi_{1}\left[s r_{i} y_{i}-c_{a}^{\star}\right]$

$r_{1}(1-x)-r_{i}\left(1-y_{i}\right)=\psi_{2}\left[r_{i}\left(1-y_{i}\right)-c_{b}^{\star}\right\rfloor$

where $\quad \psi_{1}=\left\{D_{S I} N_{S}\left(k_{m}\right)_{a} S_{b}\right\} /\left\{D_{F O} N_{F}\left(Q_{a} / d\right)\right\}$

and

$$
\psi_{2}=\left\{D_{S I} N_{S}\left(k_{m}\right)_{b} S_{b}\right\} /\left\{D_{F O} N_{F}\left(Q_{b} / d\right)\right\}
$$

Solving Eqs. (2-77) and $(2-78)$ for $y_{i}$ and $r_{i}$ in terms of $x$ and $r_{1}$, one gets locally

$y_{i}=\left(\psi_{1} c_{a}^{\star}+r_{1} x\right) /\left(r_{i}\left(1+\psi_{1} s\right)\right\}$

and $\gamma_{i}=\frac{\left[1+\psi_{1} s\right]\left[\gamma_{1}(1-x)+\psi_{2} C_{b}^{\star}\right]+\left(\psi_{2}+1\right)\left(\psi_{1} C_{a}^{\star}+r_{1} x\right)}{\left(1+\psi_{1} s\right)\left(\psi_{2}+1\right)}$

Using above two equations for $y_{i}$ and $r_{i}$, the nonlinear coupled differential Eqs. $(2-62),(2-63),(2-64),(2-65),(2-73)$ and $(2-74)$ have to be solved simultaneously with the following boundary conditions:

$S=0 \Rightarrow C_{a}^{\star}=C_{a w}^{\star} ; C_{b}^{\star}=C_{b w}^{\star}$

$S=1 \Rightarrow L^{*}=L_{f}^{*}, x=x_{f} ; r_{1}=r_{1 f} ; r_{W}=r_{W f}$

The quantities $\mathrm{C}_{\mathrm{aw}}^{\star}$ and $\mathrm{C}_{\mathrm{bw}}^{\star}$ are the dimensionless dissolved $\mathrm{CO}_{2}$ and $\mathrm{N}_{2}$ (or $\mathrm{CH}_{4}$ ) concentrations in fresh water, respectively. The value of $\mathrm{C}_{b w}^{\star}$ is equal to zero 
for the particular case of $\mathrm{CH}_{4}$.

DETERMINATION OF EPFECTIVE MEMBRANE THICKNESS IN A HFCLM PRRMEATOR

Effective membrane thickness for each permeator is determined from pure component permeation runs under conventional mode of operation with pure $\mathrm{CO}_{2}$ as feed gas and water as membrane liquid. For pure $\mathrm{CO}_{2}$ permeation, the governing differential equations for conventional mode of operation, where the permeate is withdrawn countercurrent to the feed stream, reduce to

$\mathrm{dL}^{\star} / \mathrm{dS}=\alpha_{\mathrm{a}}\left[\mathrm{r}_{1}-\mathrm{r}_{2}\right]$

$d V^{\star} / d S=\theta \alpha_{a}\left[r_{1}-r_{2}\right]$

$d r_{1} / d S=\beta \mu_{F}^{\star} L^{\star} / r_{1}$

$d r_{2} / d S=-\beta\left(D_{F I} / D_{S I}\right)^{4} \mu_{P}^{\star} v^{\star} / r_{2}$

The boundary conditions for the above set of equations are :

at $S=0 \Rightarrow V^{\star}=0\left(=V_{w}^{\star}\right)$

and

at $S=1 \Rightarrow L^{*}=L_{f}^{*} ; r_{1}=r_{1 f} ; r_{2}=r_{2 f}$

Since the permeability value of $\mathrm{CO}_{2}$ through water is known, Eqs. (2-85) to (2-88) are solved simultaneously for different values of effective membrane thickness (d). The correct value of $d$ should match the numerical results with experimental permeation rate.

DETERMINATION OP PERMEABILITY OF A NONREACTIVE SPECIES THROUGH AN AQUEOUS SOLUTION OP DRA

The permeability of $\mathrm{CO}_{2}$ through an aqueous solution of DEA may be determined from the facilitated transport analysis, described later. However, the permeability of a nonreactive gas species (e.g., $\mathrm{N}_{2}$ or He) through an aqueous solution of DEA is determined from pure component permeation runs under 
conventional mode of operation. The governing differential equations for the i-th species in dimensionless form under conventional mode of operation, where the permeate stream is withdrawn cocurrent to the feed stream, can be written as follows (with the coordinate being measured from feed entrance along the permeator):

$\mathrm{dL}^{\star} / \mathrm{dS}=-\alpha_{i}\left[r_{1}-r_{2}\right]$

$d V^{\star} / d S=\theta \quad \alpha_{i}\left[r_{1}-r_{2}\right]$

$d r_{1} / d S=-\beta \mu_{F}^{*} L^{*} / r_{1}$

$d r_{2} / d S=-\beta\left(D_{F I} / D_{S I}\right)^{4} \mu_{P}^{\star} V^{\star} / \gamma_{2}$

The boundary conditions for the above set of equations are :

at $S=0 \Rightarrow L^{*}=L_{E}^{*} ; r_{1}=r_{1 f} ; V^{*}=0\left(=V_{w}^{*}\right)$

and

at $S=1 \Rightarrow r_{2}=r_{2 w}$

Since the effective membrane thickness of the permeator is known, the above Eqs. (2-91) to (2-94) are solved simultaneously for different values of species permeability. The correct value of permeability should match the numerical results with the experimental permeation rate.

ANALYSIS OP PACILITATED TRANSPORT OP $\mathrm{CO}_{2}$ THROUGH AN AQUEOUS SOLUTION OF DEA

In the aqueous solution of $\mathrm{DEA}$ the following reactions occur with $\mathrm{CO}_{2}$ :

$$
\begin{aligned}
\mathrm{CO}_{2}+\mathrm{R}_{2} \mathrm{NH} & =\mathrm{R}_{2} \mathrm{NCOO}^{-}+\mathrm{H}^{+} \\
& \mathrm{k}_{-\mathrm{Am}} \\
\mathrm{R}_{2} \mathrm{NH}+\mathrm{H}^{+} & =\mathrm{R}_{2} \mathrm{NH}_{2}^{+}
\end{aligned}
$$

with the overall reaction being 


$$
\mathrm{CO}_{2}+2 \mathrm{R}_{2} \mathrm{NH}=\mathrm{R}_{2} \mathrm{NCOO}^{-}+\mathrm{R}_{2} \mathrm{NH}_{2}^{+}
$$

Here DEA is represented as $R_{2} \mathrm{NH}$. Since reaction (2-98) is considerably faster compared to reaction (2-97) (Danckwerts and Sharma, 1966), it is assumed to be at equilibrium. Thus reaction (2-97) controls the transport (Donaldson and Nguyen, 1980), and the rate of reaction can be written as (Blanc and Demarais, 1984)

$$
\mathrm{r}_{\mathrm{CO}_{2}}=k_{\mathrm{Am}}\left[\mathrm{R}_{2} \mathrm{NH}\right]\left[\mathrm{CO}_{2}\right]-\frac{k_{\mathrm{Am}}}{\mathrm{K}_{\mathrm{Am}}}\left[\mathrm{R}_{2} \mathrm{NCOO}^{-}\right]\left[\mathrm{H}^{+}\right]
$$

where the bracket designates the molar concentration and

$$
K_{A m}=\frac{\left[\mathrm{R}_{2} \mathrm{NCOO}^{-}\right]\left[\mathrm{H}^{+}\right]}{\left[\mathrm{R}_{2} \mathrm{NH}\right]\left[\mathrm{CO}_{2}\right]}
$$

Further the equlibrium constant of the reaction (2-98) can be written as

$$
K_{P}=\frac{\left[\mathrm{R}_{2} \mathrm{NH}_{2}^{+}\right]}{\left[\mathrm{R}_{2} \mathrm{NH}\right]\left[\mathrm{H}^{+}\right]}
$$

At steady state, local $\mathrm{CO}_{2}$ balance leads to

$$
D_{1 \mathrm{Am}} \frac{\mathrm{d}^{2} \cdot\left[\mathrm{CO}_{2}\right]}{d z^{2}}={ }^{\mathrm{r}} \mathrm{CO}_{2}
$$

Neglecting $\left[\mathrm{H}^{+}\right]$contribution, electroneutrality condition at any point can be written as

$$
\left[\mathrm{R}_{2} \mathrm{NCOO}^{-}\right]=\left[\mathrm{R}_{2} \mathrm{NH}_{2}^{+}\right]
$$

Thus for free amine 


$$
D_{R_{2} N H} \frac{d^{2}\left[R_{2} N H\right]}{d z^{2}}=2 \mathrm{r}_{C O_{2}}
$$

Assuming equal diffusivity of all the species other than $\mathrm{CO}_{2}$ leads to

$$
\left[\mathrm{R}_{2} \mathrm{NH}\right]+\left[\mathrm{R}_{2} \mathrm{NCOO}^{-}\right]+\left[\mathrm{R}_{2} \mathrm{NH}_{2}^{+}\right]=\mathrm{C}_{\mathrm{T}}
$$

where $C_{T}$ is the initial amine concentration.

Combining Eqs. (2-104) and (2-106) one can get

$$
\left[\mathrm{R}_{2} \mathrm{NH}_{2}^{+}\right]=\left(\mathrm{C}_{\mathrm{T}}-\left[\mathrm{R}_{2} \mathrm{NH}\right]\right) / 2
$$

Combining Eqs. (2-100), (2-102) and (2-107) and expressing the reaction rate in terms of dissolved carbon dioxide and free amine we get

$$
r_{\mathrm{CO}_{2}}=k_{A m}\left[R_{2} \mathrm{NH}\right]\left[\mathrm{CO}_{2}\right]-\frac{k_{A m}\left(\mathrm{C}_{\mathrm{T}}-\left[\mathrm{R}_{2} \mathrm{NH}\right]\right\}^{2}}{4 \mathrm{k}_{\mathrm{Am}} \mathrm{K}_{\mathrm{P}}\left[\mathrm{R}_{2} \mathrm{NH}\right]}
$$

Equation (2-103) can now be expressed as

$$
D_{1 A m} \frac{d^{2}\left[\mathrm{CO}_{2}\right]}{d z^{2}}=k_{A m}\left[R_{2} \mathrm{NH}\right]\left[\mathrm{CO}_{2}\right]-\frac{k_{A m}\left\{C_{T}-\left[R_{2} \mathrm{NH}\right]\right\}^{2}}{4 \mathrm{k}_{A m} K_{P}\left[\mathrm{R}_{2} \mathrm{NH}\right]}
$$

Similarly for free amine

$$
\begin{aligned}
D_{R_{2} N H} \frac{d^{2}\left[R_{2} N H\right]}{d z^{2}} & =2{ }^{C_{C O}} \\
& =2 k_{A m}\left[R_{2} N H\right]\left[\mathrm{CO}_{2}\right]-\frac{k_{A m}\left[C_{T}-\left[R_{2} N H\right]\right\}^{2}}{2 k_{A m} K_{P}\left[R_{2} N H\right]}
\end{aligned}
$$


Equations $(2-109)$ and $(2-110)$ are to be solved along with the following boundary conditions

$$
\begin{aligned}
& \text { at } \quad \mathrm{z}=0 \quad\left[\mathrm{CO}_{2}\right]=\mathrm{H}_{1 \mathrm{Am}} \mathrm{P}_{\mathrm{u}} ; \mathrm{d}\left[\mathrm{R}_{2} \mathrm{NH}\right] / \mathrm{d} z=0 \\
& \text { at } \quad \mathrm{z}=\mathrm{d} \quad\left[\mathrm{CO}_{2}\right]=\mathrm{H}_{1 \mathrm{Am}} \mathrm{P}_{\mathrm{d}} ; \mathrm{d}\left[\mathrm{R}_{2} \mathrm{NH}\right] / \mathrm{dz}=0
\end{aligned}
$$

where $\mathrm{H}_{1 \mathrm{Am}}$ is the Henry's law constant for $\mathrm{CO}_{2}$ in aqueous DEA solution; $\mathrm{P}_{u}$ and $\mathrm{P}_{\mathrm{d}}$ are the upstream and downstream partial pressures of $\mathrm{CO}_{2}$ across the liquid membrane, respectively.

Equations (2-109) and $(2-110)$ as well as the boundary conditions can be made dimensionless as follows

$$
\begin{aligned}
& \frac{d^{2} C_{3}}{d \xi^{2}}=q_{1} c_{3} c_{4}-q_{2} \frac{\left(1-C_{4}\right)^{2}}{c_{4}} \\
& \frac{d^{2} C_{4}}{d \xi^{2}}=q_{3} c_{3} c_{4}-q_{4} \frac{\left(1-C_{4}\right)^{2}}{C_{4}}
\end{aligned}
$$

where

$$
\begin{aligned}
& \mathrm{C}_{3}=\left[\mathrm{CO}_{2}\right] /\left(\mathrm{H}_{1 \mathrm{Am}} \mathrm{P}_{\mathrm{u}}\right) ; \mathrm{C}_{4}=\left[\mathrm{R}_{2} \mathrm{NH}\right] / \mathrm{C}_{\mathrm{T}} ; \xi=(\mathrm{z} / \mathrm{d}) \\
& \mathrm{q}_{1}=\mathrm{k}_{\mathrm{Am}} \mathrm{C}_{\mathrm{T}} \mathrm{d}^{2} / \mathrm{D}_{1 \mathrm{Am}} \\
& \mathrm{q}_{2}=\mathrm{k}_{A m} \mathrm{C}_{\mathrm{T}} \mathrm{d}^{2} /\left(4 \mathrm{~K}_{\mathrm{Am}} \mathrm{K}_{\mathrm{P}} \mathrm{D}_{1 \mathrm{Am}} \mathrm{H}_{1 \mathrm{Am}} \mathrm{P}_{\mathrm{u}}\right) \\
& \mathrm{q}_{3}=2 \mathrm{k}_{\mathrm{Am}} \mathrm{H}_{1 \mathrm{Am}} \mathrm{P}_{\mathrm{u}} \mathrm{d}^{2} / \mathrm{D}_{\mathrm{R}_{2} \mathrm{NH}} \\
& \mathrm{q}_{4}=\mathrm{k}_{A m} \mathrm{~d}^{2} /\left(2 \mathrm{~K}_{A m} \mathrm{~K}_{\mathrm{P}} \mathrm{D}_{\mathrm{R}_{2} \mathrm{NH}}\right)
\end{aligned}
$$

Equations (2-111) and (2-112) are to be solved along with the following boundary conditions 


$$
\begin{array}{ll}
\xi=0 \Rightarrow \quad C_{3}=1 ; & \mathrm{dC}_{4} / \mathrm{d} \xi=0 \\
\xi=1 \Rightarrow \mathrm{C}_{3}=\mathrm{P}_{\mathrm{d}} / \mathrm{P}_{\mathrm{u}} ; & \mathrm{dC}_{4} / \mathrm{d} \xi=0
\end{array}
$$

Once the solution for $\mathrm{C}_{3}$ and $\mathrm{C}_{4}$ are known, the facilitation factor $\mathrm{F}_{\mathrm{Am}}$ and the effective permeability of $\mathrm{CO}_{2}$ corresponding to the partial pressures of $\mathrm{CO}_{2}\left(\mathrm{P}_{u}\right.$ and $P_{d}$ ) can be calculated as follows:

$$
\begin{aligned}
& \text { Total Flux }=\mathrm{N}_{\mathrm{CO}_{2}}+\frac{1}{2} \mathrm{~N}_{\mathrm{R}_{2} \mathrm{NH}} \\
& \mathrm{F}_{\mathrm{Am}}=\frac{1}{2} \frac{\mathrm{D}_{2} \mathrm{NH} \mathrm{C}_{\mathrm{T}}\left\{\mathrm{C}_{4}(1)-\mathrm{C}_{4}(0)\right\}}{\mathrm{D}_{1 \mathrm{Am}} \mathrm{H}_{1 \mathrm{Am}}\left(\mathrm{P}_{\mathrm{u}}-\mathrm{P}_{\mathrm{d}}\right)} \\
& Q_{1 \mathrm{Am}}=\mathrm{D}_{1 \mathrm{Am}} \mathrm{H}_{1 \mathrm{Am}}\left(1+\mathrm{F}_{\mathrm{Am}}\right)
\end{aligned}
$$

\section{SIMULATION TECENIQUBS FOR SBPARATION PROBLEHS IN GFCLM PGRMBATOR}

The gas separation problems in HFCLM permeator under all four modes involved ordinary nonlinear coupled differential equations. These are all boundary value (BV) problems as the feed and sweep (or permeate) gas or sweep liquid stream flows in a countercurrent fashion in all cases. Thus the solution procedures are the same for all modes of operation. Determination of effective membrane thickness as well as pure component permeability from pure component permeation runs also involve the solution of boundary value problems.

IMSL subroutine DVCPR was used to solve the BV problems. The subroutine DVCPR is based on PASVA3 program (Pereyra, 1978), a finite difference method. However, to use the routine successfully one had to supply an initial estimate for each of the dependent variables at the selected grid points along the domain of the independent variable. The method of generating the initial guess was the same in all the problems. The system of differential equations was solved as an initial value problem by imposing the conditions of cocurrent flow pattern and negligible pressure drop in the sweep (or permeate) side. IMSL routine DVEKK was used to solve the initial value problem. The routine uses 
Runge Kutta formula of order 5 and 6 developed by Verner (Jackson et al., 1976). Once the initial value problem was solved then the estimates were used for subsequent solution of the actual BV problem by DVCPR routine (Sengupta, 1985; Majumdar, 1986).

\section{SOLUTION PROCEDURE POR FACILITATED TRANSPORT ANALISIS}

The analysis of facilitated transport of $\mathrm{CO}_{2}$ through an aqueous solution of DEA results in two second order ordinary differential equations. These are highly nonlinear boundary value problems and have enjoyed considerable attention since no exact solutions exist. Two approaches have been used for the solution; semianalytical and numerical. The semianalytical methods are based on matched asymptotic expansions (Goddard et al., 1970; Kruezer and Hoofd, 1970; Smith et al., 1973), regular perturbation method (Suchdeo and Schultz, 1974), and techniques involving other approximations to the differential equations (Friedlander and Keller, 1965; 0tto and Quinn, 1971; Donaldson and Quinn, 1975; Smith and Quinn, 1979; Hoofd and Kruezer, 1981). The numerical methods involve the quasilinearization technique (Kutchai et al., 1970; Suchdeo and Schultz, 1974), a finite difference technique with nonuniform mesh size (Nedelman and Rubinow, 1981) and a technique based on the Galerkin method (Ward, 1970). More recently Jain and Schultz (1982) solved the problem numerically by a technique based on orthogonal collocation on finite elements introduced by Carey and Finlayson (1975). However, the solution procedure is more complex. A simpler numerical solution procedure was adopted here.

Complete numerical solution of the facilitated transport equations involved the following steps. At first, the two second order differential equations were converted into four first order ordinary differential equations. Then the system of equations were solved by using IMSL routine DVCPR. In this particular case initial guess was generated by assuming that all the reactions were at equilibrium. Once the initial guesses were generated for all the variables, the problem was solved by DVCPR routine (Guha, 1989).

\section{GENERALIZED TWO DIMENSIONAL MODEL FOR MEMBRANE THICKNESS DETERMINATION}

A generalized computer program has been developed to estimate the effective 
membrane thickness (EMT) for the HFCLM (hollow-fiber-contained liquid membrane) module in which no chemical reactions were occurring. The computer program based on a 2-dimensional diffusion analysis presented in the NYSERDA Report 87-10 could not be used directly for calculation of an EMT for different outer diameters (O.D.) of fibers without modification. It is useful to generalize the computer program so that EMT can be calculated immediately whenever the outside diameter of the fiber is given. This will allow module design and analysis for other fiber sizes that may be obtained in future.

To achieve this goal, it is necessary to develop several techniques in addition to the algorithm presented in NYSERDA Report No. 87-10. First, the boundary points are created automatically along the given circle and the identification numbers are assigned to each boundary grid point depending on the type as shown in Figure 2-4. Boundary grid points can be grouped into four categories. Variables "a" and "b" refer to the horizontal fractional intercept and the vertical fractional intercept, respectively of the unit grid dimension. EPS indicates the tolerance of " $a$ " and " $b$." When the magnitudes of " $a$ " and " $b$ " are both less than the value of EPS, the grid point is located on the curved boundary. Values of "a" and "b" are computed at each boundary grid point and are incorporated in calculating the dimensionless concentration. Since one square pitch cell is constructed of four quarter circles of radii $R_{1}, R_{2}, R_{3}$ and $R_{4}$, as shown in Figure 2-5, four subroutines were developed.

Second, once all the boundary points are identified, the interior points are identified with ID number zero. The next step is to solve the governing partial differential equation. For computer calculations, a finite difference method is used with Liebmann's iterative scheme (Chow, 1979). Such iteration formula at the interior grid point $(i, j)$ can be written as

$\left.C_{i, j}^{n+1}=(1 / 4) * 1 C_{i-1, j}^{n+1}+C_{i+1, j}^{n}+C_{i, j-1}^{n+1}+C_{i, j+1}^{n}\right]$

where the superscripts denote the number of iterations. At the boundary points as shown in Figure 2-4, the corresponding iteration formula is

$$
\begin{aligned}
c_{i, j}^{n+1}=\left\{C_{A} /\{a(a+1)\}+\right. & C_{B} /\{b(b+1)\} \\
& \left.+C_{i-1, j}^{n+1} /(a+1)+C_{i, j+1}^{n} /(b+1)\right] *\{a b /(a+b)\}
\end{aligned}
$$




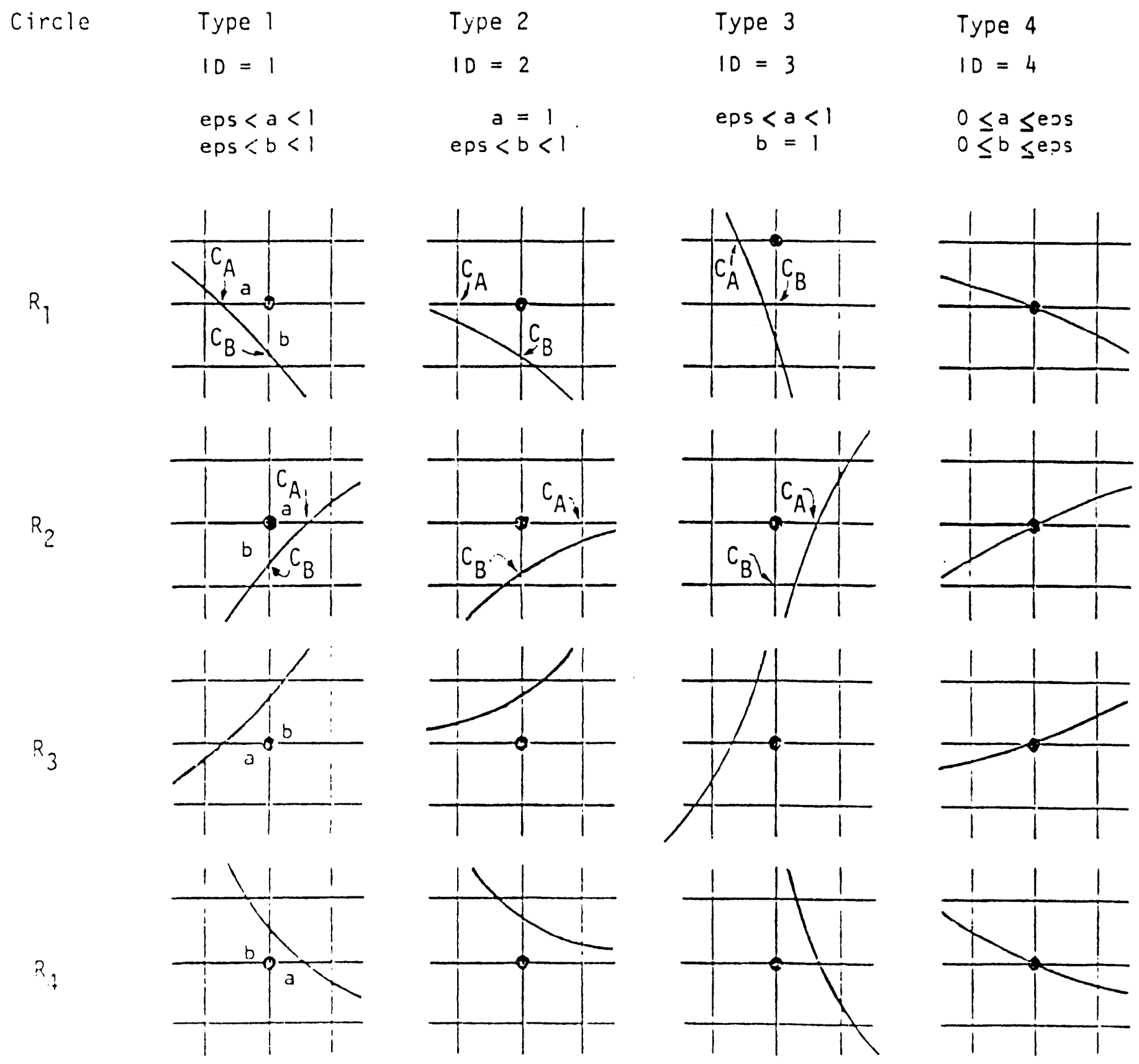

Type 0

$10=0$

Interior Grid Points

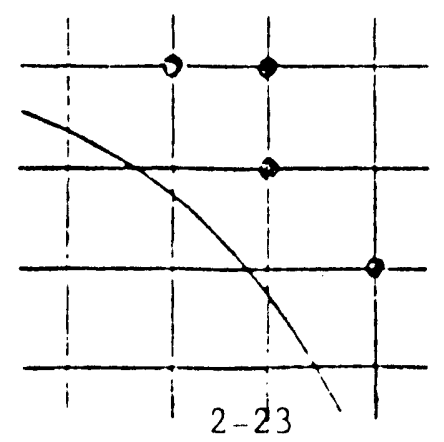

FIGURE $\quad 2-4$

TYPES OF BOUNDARY POINTS 


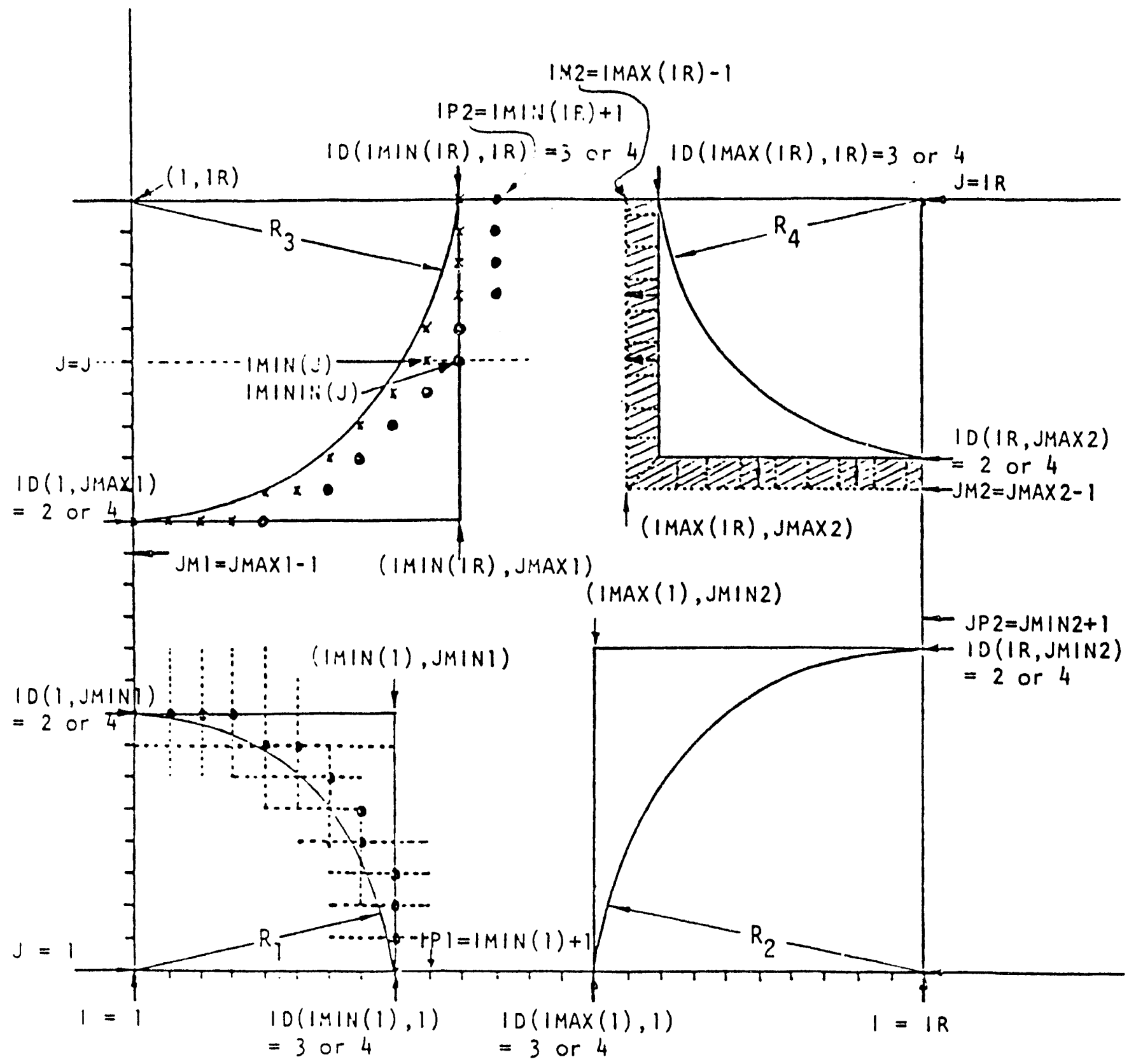

FIGURE 2-5

NATURE OF A CELL 
where $C_{A}$ and $C_{B}$ refer to the dimensionless concentrations of the neighboring points shown in Figure 2-4. To start the iteration, a constant value of zero is first guessed for the dimensionless concentration at all interior points. With the proper boundary conditions for $C$ shown in Figure 2-6, a new value of $C$ is computed from the above equation at each grid point. The absolute value of the difference between this and the previous value of $\mathrm{C}$ at the same grid point is calculated and is added to the value of a variable called ERROR, whose starting value at the beginning of an iteration is zero. At the end of one iteration, this value is compared with maximum allowable value of the total error.

Third, the number of grid points can be adjusted so that the possible error could be reduced to the minimum. Then the number of grid points are increased, not only can the boundary grid points be located near the curved boundary, but the accuracy of calculation for the interior points can also be increased. In such a fashion, che dimensionless concentration can be obtained at all grid points.

Finally, the total rate of solute transport per cell per unit length of the permeator can be obtained by a subroutine called 'FLUX.' The effective membrane thickness can be calculated in the same subroutine. The complete listing of the program is given in the Appendix.

The computer program is first applied for the EMT estimation of a permeator module which is made of only one kind of fibers while the fiber size varied. It can be utilized also for estimation of the EMT for a permeator made of mixed fibers; for example, when two different sizes of fibers are used where fibers of one size act as sweep fibers and fibers of other size act as feed fibers.

A schematic diagram of a unit cell consisting of two different sizes of fibers is shown in Figure 2-7. From the geometrical configuration of the permeators \#6 and $\# 7$ (feed fibers: number $=300, O D=150 \mu \mathrm{m}$ ind sweep fibers: number $=155$, $O D=290 \mu \mathrm{m}$ ), the pitch of the square configuration cell (assuming the rhombus to be replaced by a square) is given by

$t=\left\{\left(D_{b}-D_{F O}\right) /\left(6 N_{S}\right)\right]\left[-\pi /(2 \sqrt{ } 2)+i\left(\pi^{2} / 8+3 \pi N_{S}\right\}\right]$ 
LEE I. FOR

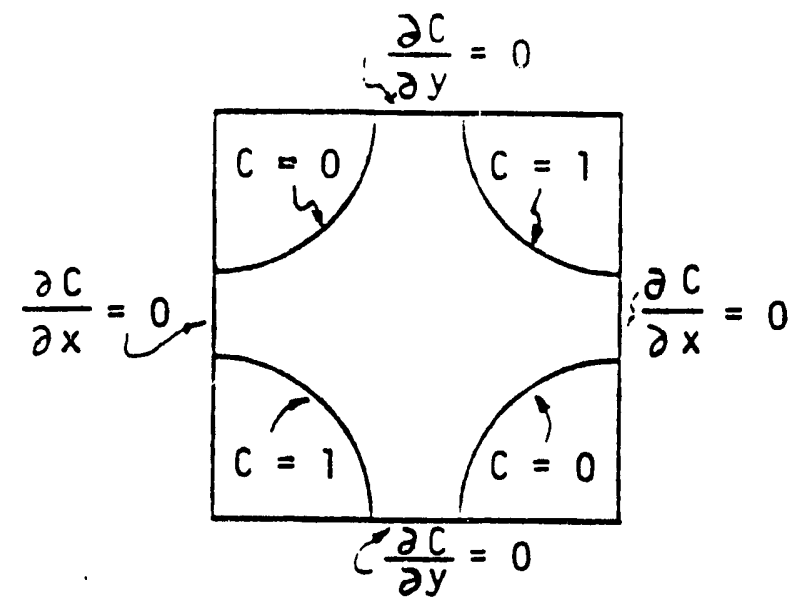

LEE 2.FOR

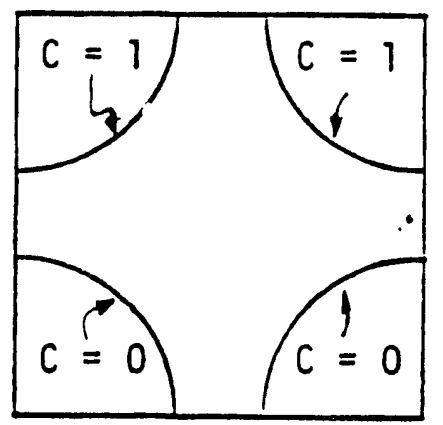

LEE3.FOR
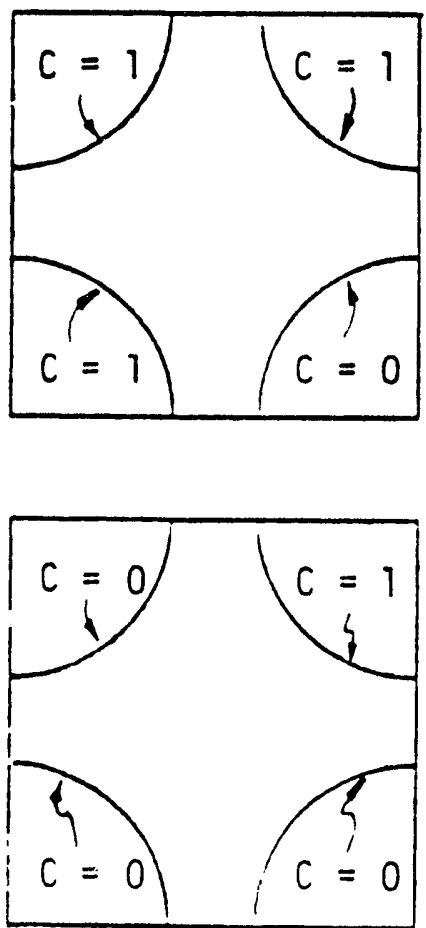

FIGURE $2-6$

TYPES OF BOUNDARY CONDITIONS 


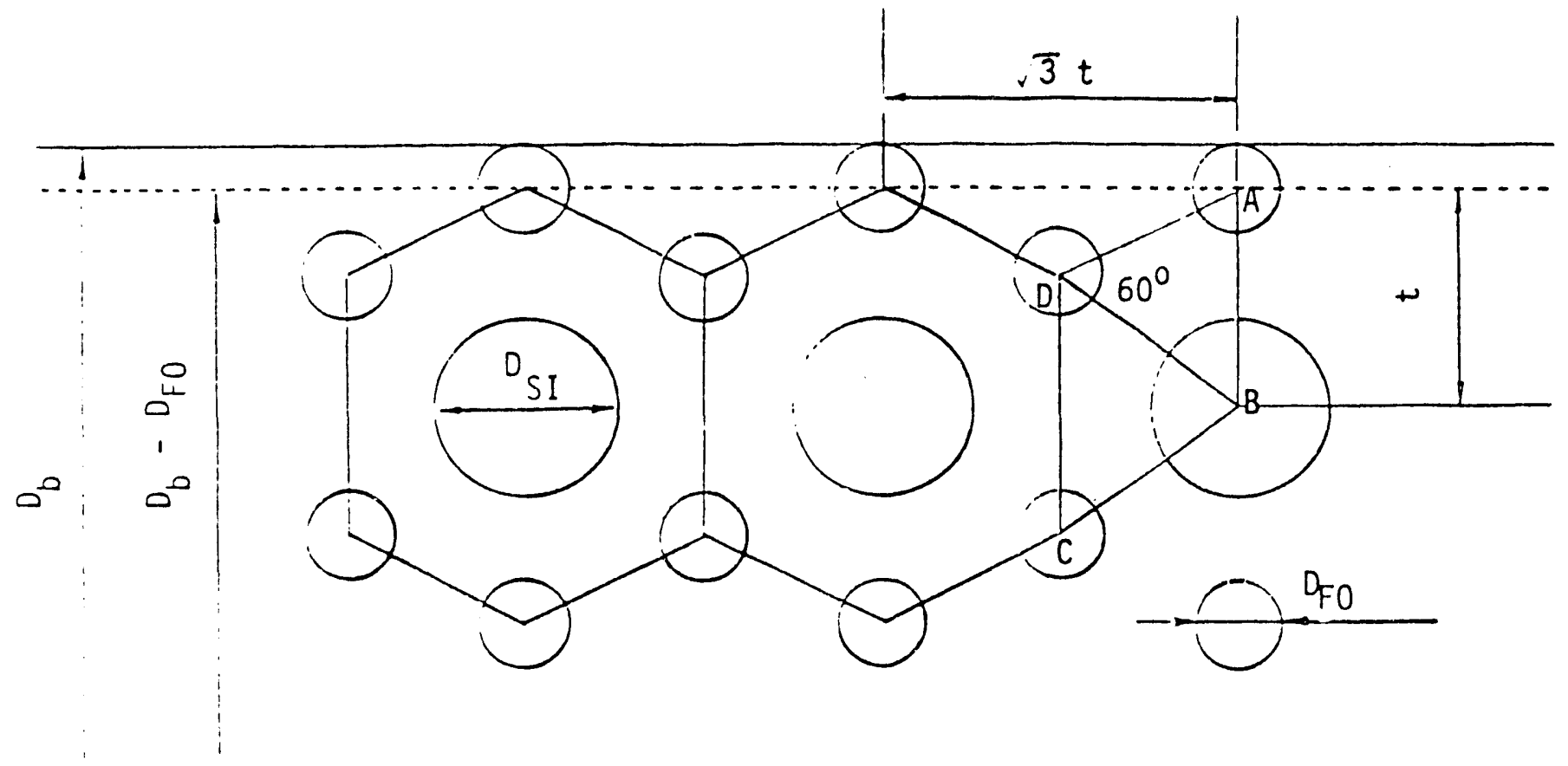

FIGURE $2-7$

SCHEMATIC REPRESENTATION OF A RHOMBOIDAL UNIT CELL IN THE MHF BUNDLE ASSEMBLY OF A MIXED FIBER CLM PERMEATOR 
Unlike permeators made from fibers of uniform size, there exists greater restrictions in the calculation of the EMT in the mixed fiber permeator. Since the bigger 0.D. fibers act as sweep fibers and the smaller 0.D. fibers act as feed fibers, there is only one possible symmetrical geometrical arrangement of mixed fibers. Namely, one square cell contains three of one quarter of feed fiber and one quarter of sweep fiber.

For the case of a permeator constructed of the fibers of uniform size, the EMT based on the feed fiber is the same as that based on the sweep fiber. However, the EMT calculated based on the feed fiber is slightly different from that estimated based on the sweep fiber for the permeator made of two kinds of fibers, because the total mass transport rate in a cell remains constant while the available surface area of sweep fiber is different from that of feed fiber in a unit cell. 


\section{Section 3}

\section{EXPERIMRNTAL}

In the present investigation, microporous hydrophobic polypropylene Celgard $\mathrm{X}-10$ hollow fibers of 100 micron ID and 240 micron ID were used for fabricating the hollow-fiber-contained. liquid membrane permeators. Both of these fibers have bubble point pressures of 150 psi with mean pore sizes of 0.03 micron and thickness of 25 micron. Thus both sizes are suitable for biogas applications, as the separation studies were conducted at an absolute pressure of 60 psi or less depending on the operating mode.

The ideal characteristics for a liquid for the purpose of a membrane in the HFCLM technique are: stable, nonfoaming, relatively low volatility, not highly corrosive and viscous; further, it must have a high solubility for one of the solutes compared with that for the other. In earlier studies of biogas purification, pure water and aqueous solution of $\mathrm{K}_{2} \mathrm{CO}_{3}$ were used as liquid membranes whereas in the present study more emphasis was given to aqueous solution of organic liquids.

\section{DETERMINATION OF VETTABILITY, BUBBLE POINTS AND BREARTHROUGH POINTS}

It was essential to develop information about certain physical properties of the liquid membrane - microporous substrate system before aqueous solution of organic liquids could be used as membranes in a HFCLM permeator. These are: wettability of the microporous membrane by the membrane liquid, breakthrough pressure and/or bubble point pressure as the case may be. The following organic liquids, monoethanolamine (MEA), diethanolamine (DEA), and 1-methyl-2-pyrrolidinone (NMP) and their aqueous solutions were subjected to such testing.

When the solvent or its solution wetted the microporous polypropylene membrane, the bubble point measurement had to be carried out. On the other hand, if the membrane was not wetted by a given solvent or solution, the solvent breakthrough measurement had to be performed. The bubble point is defined as the pressure of air or nitrogen gas required to force the solvent from the largest wetted pore of a membrane. Generally, the larger the pore, the 
lesser is the pressure required to expel the liquid. The solvent breakthrough pressure is the pressure required to transmit the solvent through the largest pore of a membrane not wetted by the solvent.

Such information was generated using Celgard 2400 flat films (Celanese Specialty Operations, Summit, N.J.), the surface characteristics of which are identical to the Celgard hollow fibers. Although the pore sizes of Celgard 2400 are somewhat smaller ( 0.02 micron) than those of the hollow fibers, it was felt that the information would be sufficient for decision making purposes. As displayed in Table 3-1, the measured breakthrough points were higher than 60 psi in Celgard 2400 for certain solutions. This means that it is safe for the pressure of liquid membrane (i.e., 20 vol\% water in NMP or 20 wt\% DEA solution in water) to be slightly higher than the highest operating pressure of gas streams (60 psia) in the permeator. There would not be any membrane liquid breakthrough into the sweep fibers under the operating conditions.

\section{PABRICATION OF SMALL-SCALE GFCLM PERMEATORS}

The fabrication procedures for first four permeators (\#1 to \#4) were described in detail in the NYSERDA Report 87-10. Five more small-scale permeators were fabricated during this investigation and were numbered sequentially (e.g., \#5 to \#9). The detailed geometrical characteristics of each permeator used in this study are given in Table 3-2. The hollow-fiber surface area in the feed side was equal to that of the permeate side in all the permeators.

Fabrication of a permeator involved preparation of fiber bundles and putting them in a shell and finally potting the ends with a resin mixture to form a tube sheet.

For permeator \#5, a 9 -ft-long mat of 300 fibers (X-10, 150 micron OD) was first prepared on a polyethylene sheet over a laboratory bench top as follows. The bobbin containing the fibers was first exposed to the exhaust from a humidifier to eliminate the static charge on the surface of the fibers. Then six fibers were taken out at a time from the bobbin; they were cut to the desired length using scissors and both ends of the fibers were attached to a strip of scotch tape. Thus $300 \mathrm{fibers,} 9 \mathrm{ft}$ long, were placed close to one another to complete 


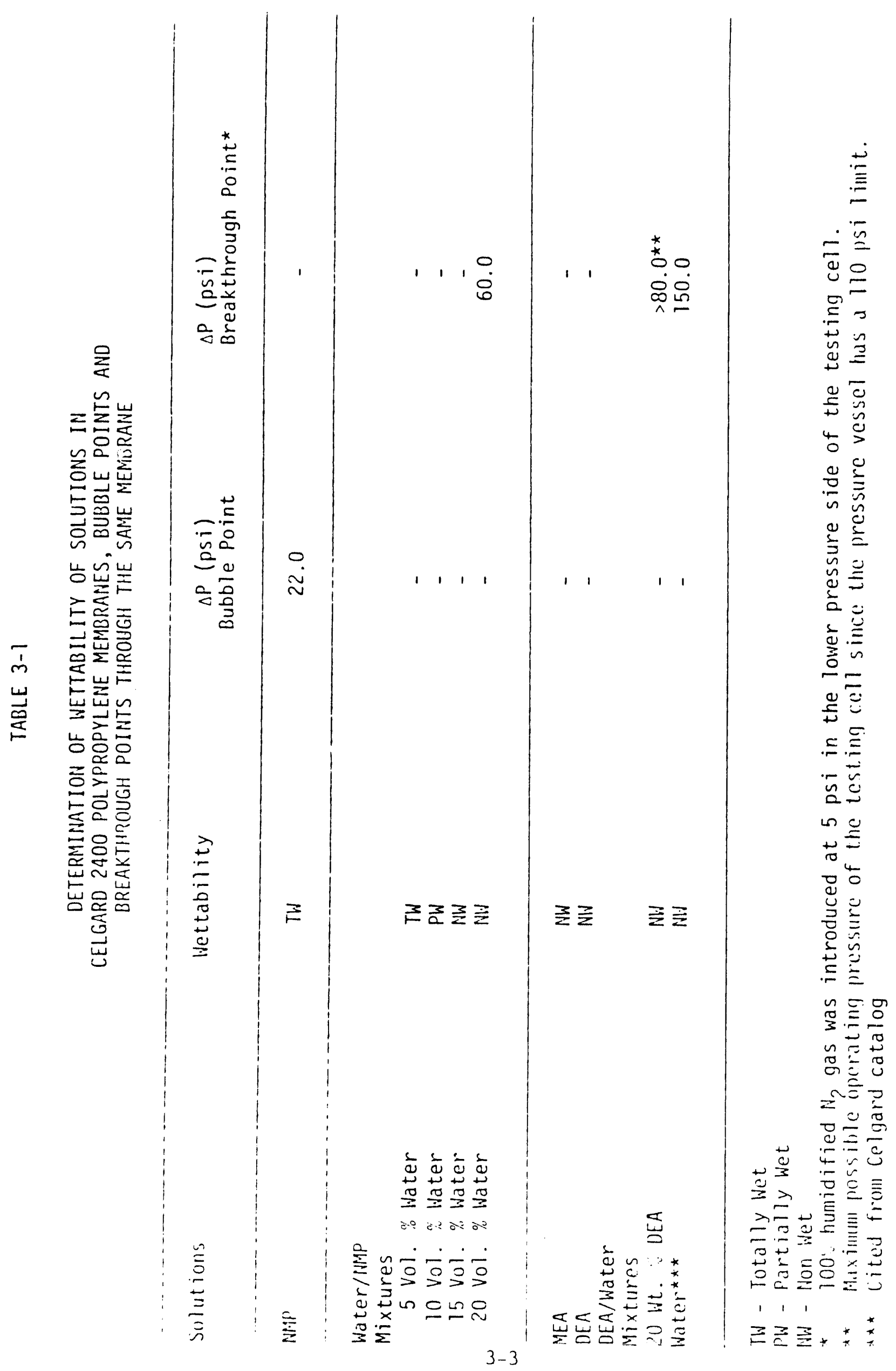


Table 3-2

DETAILED GEOMETRICAL CHARACTERISTICS OF

SMALL-SCALE HFCLM PERMEATORS

\begin{tabular}{ccccccc}
\hline $\begin{array}{c}\text { Permeator } \\
\#\end{array}$ & $\begin{array}{c}\text { Active } \\
\text { Length } \\
\text { inch }\end{array}$ & $\begin{array}{c}\text { Feed } \\
\text { Total } \\
\text { No. }\end{array}$ & $\begin{array}{c}\text { Fiber } \\
\text { micron }\end{array}$ & $\begin{array}{l}\text { Permeate } \\
\text { Total } \\
\text { No. }\end{array}$ & $\begin{array}{c}\text { Fiber } \\
\text { IDicron }\end{array}$ & $\begin{array}{c}\text { Void } \\
\text { mpace } \\
\%\end{array}$ \\
\hline 3 & 62 & 300 & $100 / 150$ & 300 & $100 / 150$ & 66.5 \\
4 & 62 & 300 & $100 / 150$ & 300 & $100 / 150$ & 63.7 \\
5 & 62 & 300 & $100 / 150$ & 300 & $100 / 150$ & 63.7 \\
6 & 24 & 300 & $100 / 150$ & 155 & $240 / 290$ & 46.7 \\
7 & 24 & 300 & $100 / 150$ & 155 & $240 / 290$ & 46.7 \\
9 & 17 & 300 & $100 / 150$ & 300 & $100 / 150$ & 63.7 \\
\hline
\end{tabular}


one mat. Once such a mat was ready, the mat ends were covered by two polyethylene sheets about 10 inches long. The polyethylene sheets were kept in place by scotch tape outside the region of the mat. Two new pieces of scotch tape were placed on the polyethylene sheets to put another 300 fibers having the same dimensions. These fibers were placed in between the fibers of the previous mat to ensure close proximity between the two sets of fibers. Thus, two sets of fibers were well mixed in the middle section but separated completely at the end by the upper polyethylene sheets.

For permeator \#6, one set of fibers consisted of 300, $X-10$ Celgard hollow fibers of 150 micron outside diameter and the other set consisted of $155, X-10$ fibers of 290 micron outside diameter. The total number of fibers in each set was chosen in such a way that the total area of permeation remained equal on both sides (feed and permeate). Preparation of the mat was done exactly in the similar fashion but the total length of each fiber taken was $4 \mathrm{ft}$ with an effective permeator length of 2 feet.

The fiber dimensions for permeator \#7 were similar to those of permeator \#6. However, the fibers utilized in this permeator were tested for leakage before preparation of the integrated fiber mat. A large number (about 400) of fibers $(\mathrm{X}-10,150 \mathrm{micron} 0 . \mathrm{D}$.$) , each about 5 \mathrm{ft}$ long, were first potted at each end in a 2-inch-long hexagonal nipple (Cajon) using an epoxy resin mixture prepared with $80: 20$ ratio of $C-4$ resin to activator D (Beacon Chemical Co., Mt. Vernon, NY). The hollow fibers were then pressurized internally with water up to a pressure of 50 psig. This type of test was done also for the other set of fibers (X-10, 290 micron 0.D.). About 300 fibers were taken for this purpose. A few fibers were found to be defective in each case and were leaking during the test. The leaky fibers were separated and discarded. The fiber mat for the permeator \#7 was then prepared with the remaining good fibers in a manner similar to that for permeator \#6.

In the case of permeators \#8 and \#9, the fibers were also pressure tested in a manner similar to that for permeator \#7. However, in both cases, fibers of 100 micron ID were used in feed as well as sweep sides. To minimize the handling of fibers one more strip of scotch tape was placed at 6 inches from both the ends, so that when the ends were potted in the nipple for pressure testing they were 
still in the form of a mat. Figure 3-1 shows two separate mats with both ends potted in the nipple. After pressure testing, leaky fibers as well as some good fibers were removed to bring the total number of fibers in each mat to exactly 300. Then the mats were cut from the nipples and one mat was placed on the top of the other to make an integrated mat with the ends being separated. The integrated mat was then rolled to prepare the fiber bundle as before. The technique for making the fiber bundle was the same for all the permeators.

Distilled water was sprayed over the mat for easy handling of the fibers. Fibers were slowly gathered together starting from the middle section and gradually approaching the end. Cotton threads (wetted) were tied loosely in the middle section as well as at the four separated ends to keep the fibers together as shown in Figure 3-2. Cotton threads were removed slowly as the fiber bundle was inserted into specially constructed permeator shell. A 1/8inch teflon pipe (I.D. 0.240 inch, 0.D. 0.406 inch; Cole Parmer, Chicago, IL) was inserted into the $1 / 2$-inch schedule 40 stainless steel pipe to make the shell. Epoxy resin was used to fill the annular space between the two concentric pipes. The pipe assembly thus constructed acted as a shell for all the new permeators. The pipe was fitted with a $1 / 2$-inch stainless steel street tee (Cajon) followed by $1 / 2$-inch stainless steel $45^{\circ} \mathrm{Y}$-fitting (NJ Engineering \& Supply Co., Passaic, NJ) at each end. The end fitting of permeator \#5 is shown in Figure $3-3$.

The side opening of the street tee was used as a shell side liquid port (entry or exit). The two openings of the Y-fittings were needed to separate the two sets of fibers. Y-fittings have distinct advantage over cross connection (used for permeators \#1 to \#4; Majumdar, 1986) as they require less bending of fibers. To each of these ends, a 2-inch-long hexagonal nipple (Cajon) connection was attached. The internal surface of the nipple was threaded in nrder to increase the metal-resin (epoxy or polyurethane resin was used for potting the fibers) bond strength.

The permeator assembly was thoroughly cleaned respectively with a soap solution, an aqueous sulphuric acid solution and water before the fiber bundle was inserted in $i t$. The shell was completely immersed in water and the fiber bundle was gently pulled through the shell assembly by using a cotton thread 


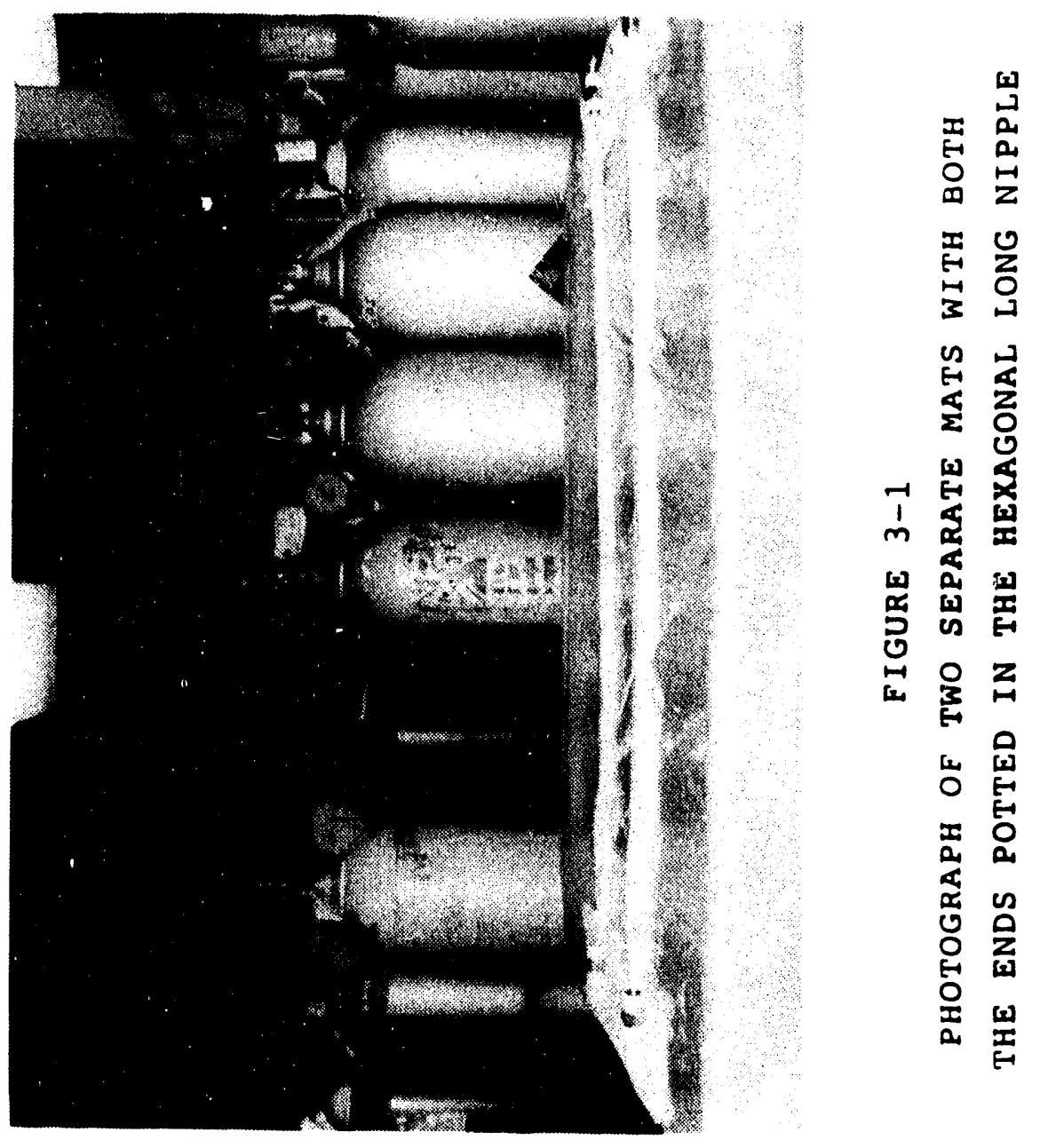




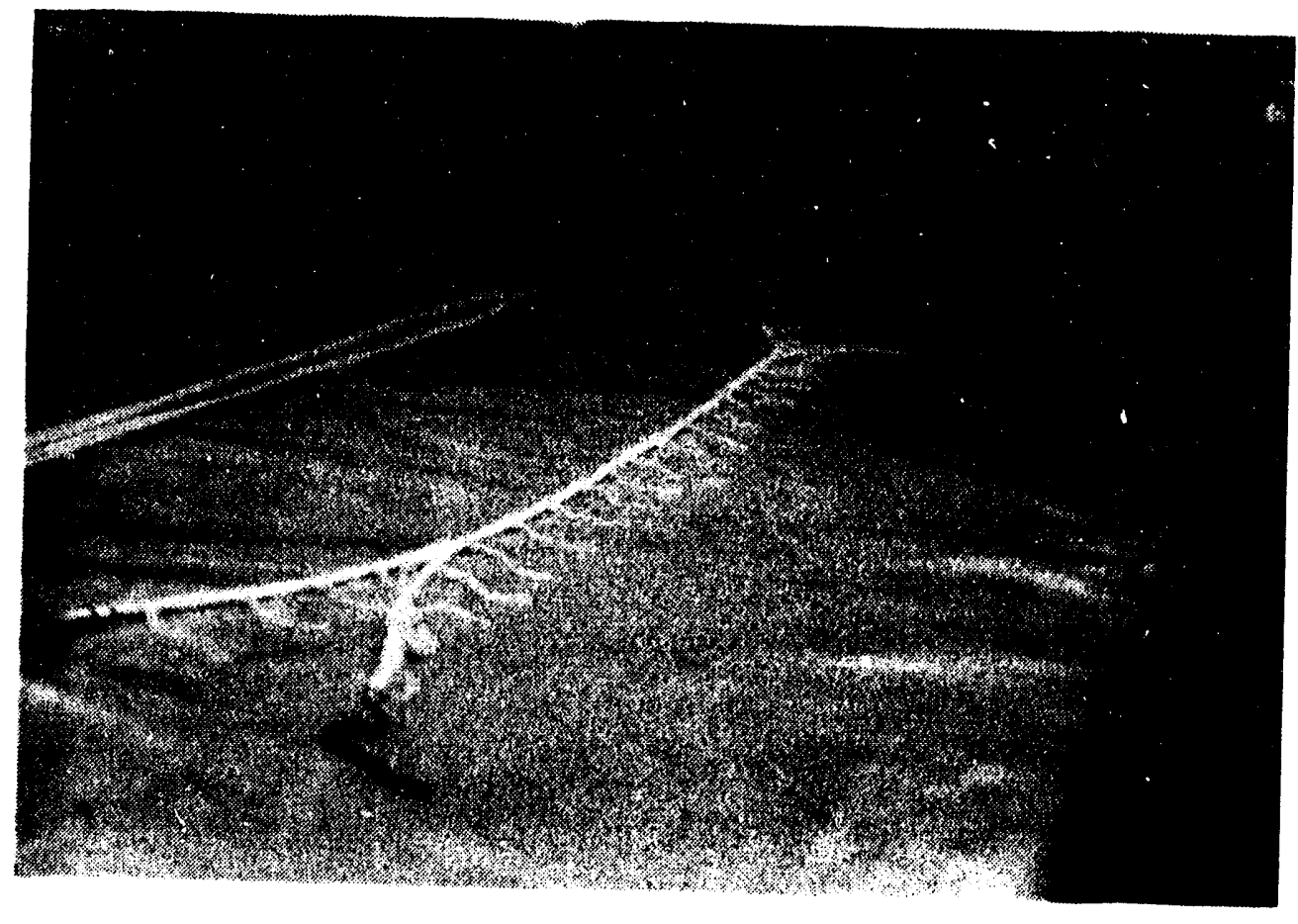

FIGURE 3-2

PHOTOGRAPH OF INTEGRATED MAT (SEPARATED AT BOTH ENDS) TIED WITH COTTON THREADS 
$: \quad \cdots$

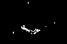

$+\quad, \quad$,

.

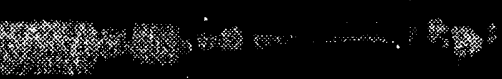

${ }^{2} y_{2}$
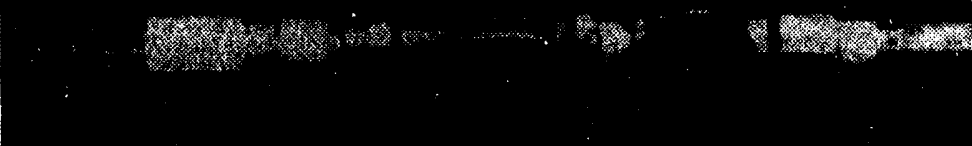

$\therefore$

FIGURE 3-3

PHOTOGRAPH SHOWING END FITTINGS OF PERMEATOR \#5 
tied at one end of the bundle. Once the bundle was through at both the ends, two sets of fibers were separated and each set was then introduced through one of the hexagonal nipple connections. The whole assembly is shown in Figure 3-4. The assembly was air dried. Before che assembly was ready for potting, a tygon tube was slid over each fiber bundle end and attached to the hexagonal nipple by using a hose clamp. The other end of the tygon tube was connected with a polypropylene Y-connection. The fiber bundle was taken out through one arm of the Y-connection; the other arm was used to pour the resin mixture. The tygon tube was then given $U$-shape and clamped in that position. The arrangement is shown in Figure 3-5. The polyurethane resin mixture prepared with a 58:42 ratio of Polycin 1670 to Vorite 1701 (Caschem Inc., Bayonne, NJ) was then poured into the $U$-tube through the free arm of the Y-connection. The resin was poured till the polyurethane level in the permeator end was raised io the middle of the nipple. Two arms of a $Y$ were potted at a time. After 34 hours the fiber bundle at the end of the nipple was exposed by cutting off the poiyurethane resin with a sharp knife. Seven more days were required for the complete curing of the resin. The configuration of such a permeator is shown in Figure 3-6.

The ends of permeators \#8 and \#9 were potted with epoxy resin (resin: C4; activator: D; ratio: 4:1; Beacon Chemical Co., Mt. Vernon, NY) instead of polyurethane. The potting technique was different from all other small-scale permeators. Eisxy resin was added through an opening made on the body of each Y-fitting. At the end of permeator construction, both the openings were plugged.

\section{FABRICATION OP LARGE-SCALE HPCLM PERMEATORS}

Three large-scale HFCLM permeators (\#10, \#11 and \#12) had been built for largescale gas purification studies. Each permeator contained a total of $7008, x-10$ Celgard hollow fibers, divided equally between the feeci and sweep sides. The $\mathrm{t}$ iber inside and outside diameters were $100 \mathrm{micron}$ and 150 micron, respectively. The effective permeation lengths for permeators \#10, \#11 and \#12 were $? 6.24 .5$ and 24.5 inches, respectively.

The $t$ iber mat preparation procedure for large-scale HFCLM permeators was somewhat different. A plexiglass sheet (size: 42"X21"X1/4") was mounted on an 


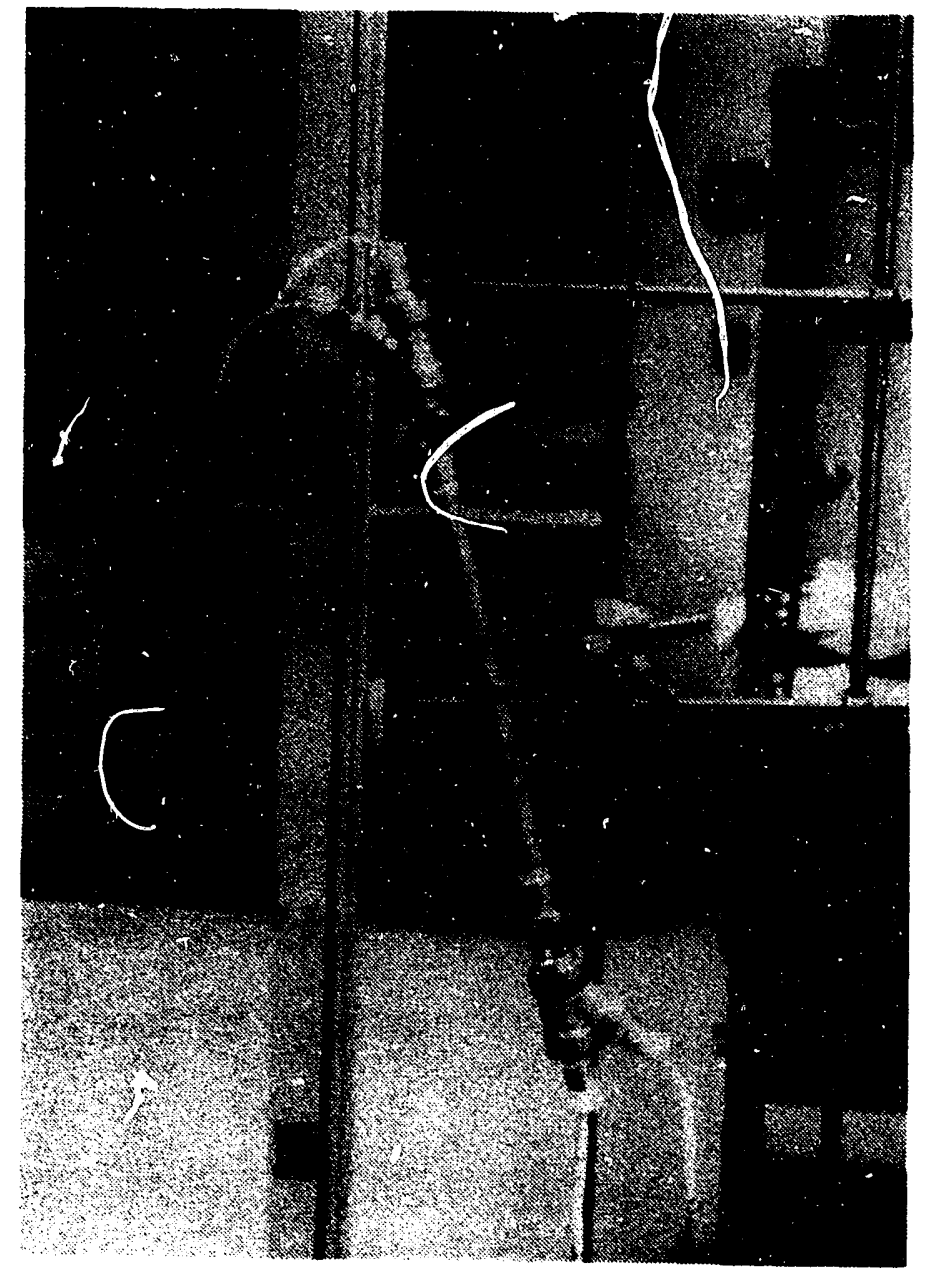

FIGURE 3-4

PHOTOGRAPH OF A PERMEATOR WITH TWO SETS OF FIBER TAKEN OUT THROUGH THE END FITTINGS 


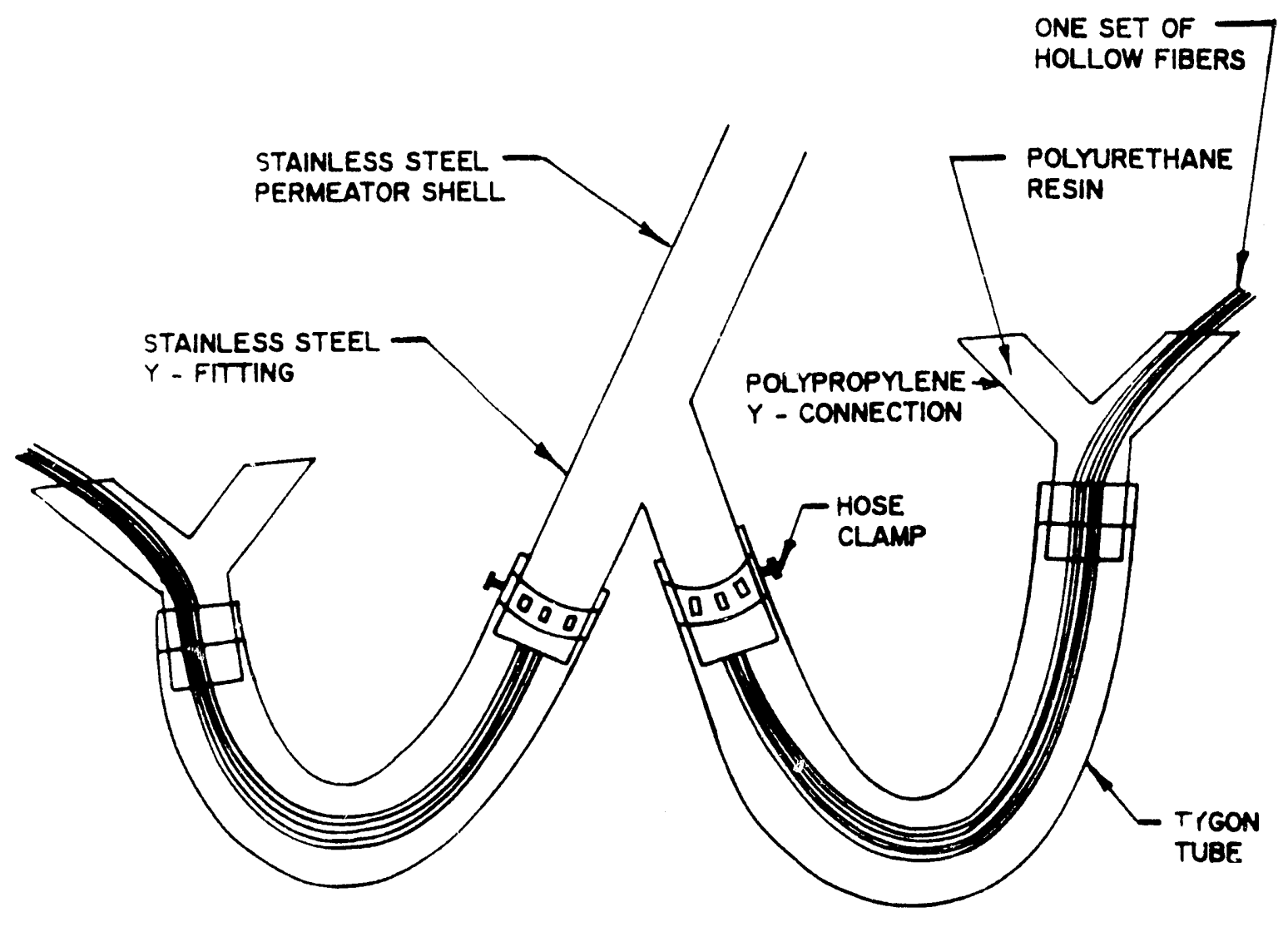

FIGURE 3-5

SCHEMATIC OF POTTING THE END OF A PERMEATOR

BY U-TUBE METHOD 


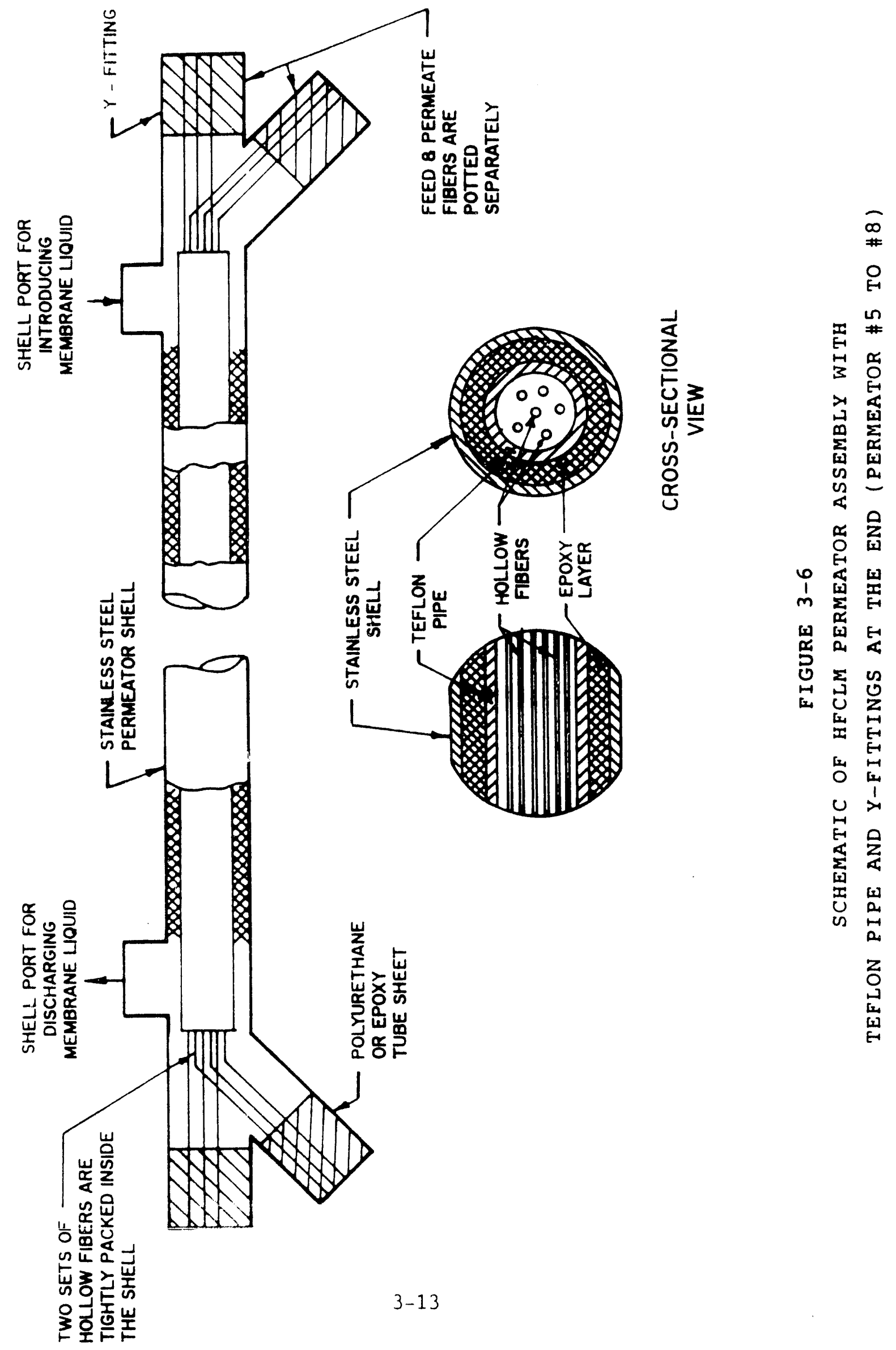


electrically driven motor with proper connections. (The connection was made in the middle of the plexiglass piece.) It was wrapped twice with a polyethylene sheet to cover sharp edges at the ends. Celgard hollow fibers from a spool were then wound around the plexiglass sheet by rotating it slowly by hand. The fiber spool was kept away from the plexiglass/gear box arrangement and was allowed to rotate on a circular shaft. Distilled water was occasionally sprayed over the fibers for easy handling. Finally, a structure was created in which two layers of fibers remained at each side of the plexiglass sheet. One layer of fibers would be used as feed fibers, whereas the other layer of fibers would serve as sweep fibers. The polyethylene sheet along with the fibers were separated from the plexiglass by gently pulling the $\mathrm{PL}$ sheet from both ends. Four cotton threads were inserted (for permeator \#10) in between the two layers of fibers. (For permeators \#11 and \#12, the cotton threads were placed and secured by means of several strips of scotch tapes on top of the polyethylene sheet at the beginning of the mat preparation procedure.) The threads were tied loosely at the ends of each fiber layer. The polyethylene sheet was then removed very carefully. At this point, one layer of fibers remained on top of the other layer. The four cotton threads were then tied very tightly and the layers were separated by cutting the fibers with a pair of scissors. The fibers were then rolled gently and slowly starting from the middle to form a 42-inch-long bundle of about 7000 fibers, keeping the four ends separated. Cotton threads were tied loosely to keep the fibers together.

A 2-ft.-long, 1-inch schedule 40 stainless steel pipe was used as the permeator shell. An epoxy mixture (C4-D) was applied to the outside surface of a teflon hollow rod (ID: $3 / 4$ inch; OD: 1 inch), which was inserted into the stainless steel shell. After complete curing of the epoxy, the pipe was fitted with a nylon connection at each end (except for permeator \#11). This connection was made in our machine shop from a 2-inch-thick nylon block. It had 3 large (size: 1 inch) openings in the form of $a^{\prime} ' Y^{\prime}$ and two small (size: $1 / 4$ inch) openings. The central opening was attached to the stainless steel pipe, whereas the other two openings were connected to two hexagonal nipples, needed to separate the two sets of fibers. One of the small openings was used as the shell liquid port and the other one was plugged. For permeator \#11, one SS 1inch cross connection was connected to each elid of the pipe. Two openings of the cross were fitted with two hexagonal nipples. The other opening would be 
connected to the membrane liquid reservoir line.

The bundle was then inserted in the shell. Two sets of fibers were separated at the ends, and each set was then introduced through one of the hexagonal nipple connections. The whole operation was carried out keeping the bundle and the shell under water. To pot the fibers, a base sheet was first prepared on the hexagonal nipple using A-2 epoxy (Beacon Chemical Co., Mt. Vernon, NY). A second layer of epoxy (C4-D) was then poured on top of the A-2 epoxy using the 1/4-inch opening of the nylon connection. The curing procedure was carried out under centrifugal force i.e., the permeator was allowed to rotate on a vertical axis at high speed. The process ensured that the space between the adjacent fibers was filled with the epoxy resin. The resin was allowed to harden for 24 hours at room temperature. The procedure was repeated for all four ends. Figure 3-7 shows the three permeators.

\section{PERMRATOR TESTING UNDER STATIC PRESSURE}

Before a new permeator was used in the gas purification loop, it was necessary to test it for any leak which could be either in the tube sheet (made of either polyurethane or epoxy resin) due to incomplete wetting of the fiber by the resin or due to a defect or defects on the fiber surface or wall. Leak testing was done in the following manner.

The permeator shell was filled with distilled water and the shell side was pressurized by a $\mathrm{N}_{2}$ cylinder, keeping the other shell side port completely closed. The pressure was increased slowly to 50 psig in steps of 10 psig. observations were made at each pressure level for any sign of water coming out through the tube sides. Finally, the pressure was maintained at 50 psig and the permeator was checked for any further leakage of water through the tube sides. This type of test was carried out for all the permeators. As expected, the larger diameter fibers in the permeators \#6 and \#7 leaked more compared to the smaller diameter fibers. Generally, the leakage rate was small in all permeators; permeator $\# 8$ had the least leakage rate among the small-scale permeators.

Static pressure testing of large-scale permeators was normally carried out 


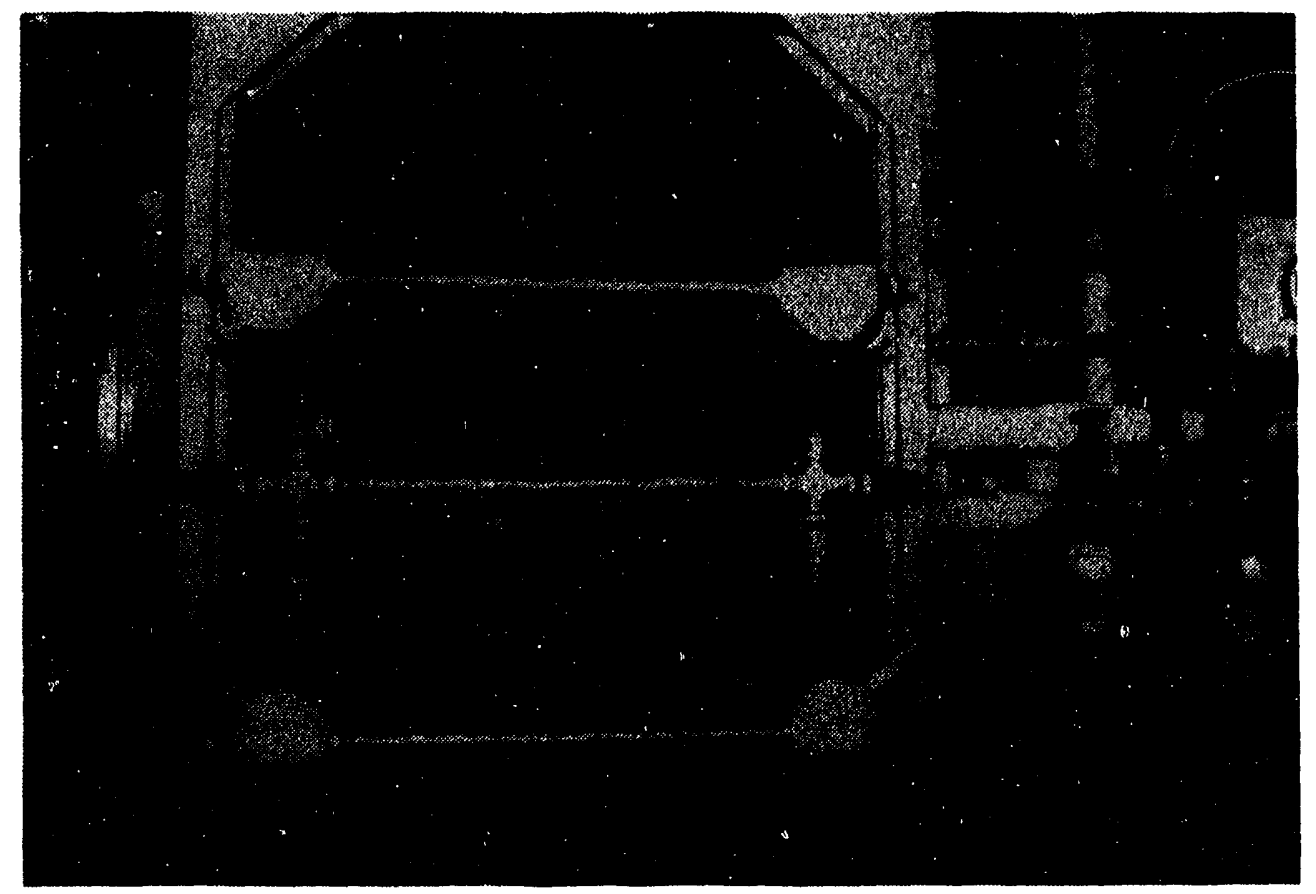

FIGURE 3-7

PHOTOGRAPH OF THREE LARGE PERMEATORS (\#10, \#11 AND \#12) 
without removing the extra length of fibers from the tube-sheet. When tested for leaks, Permeator \#12 was found to be leaking severely through one of the tube-sheets $\left(90^{\circ}\right.$ end of a Cross) even at a low pressure of 5 psig. The permeator was allowed to dry thoroughly under vacuum and another layer of epoxy resin was applied through the shell liquid opening. The permeator was tested again and no improvement was seen. After drying the permeator, a hexagonal nipple with a coupler was attached to the leaky tube-sheet end and epoxy resin was poured from the top onto the extra length of fibers. The resin was allowed to harden for 24 hours and the permeator was tested again up to a pressure level of $50 \mathrm{psig.} \mathrm{No} \mathrm{leakage} \mathrm{of} \mathrm{water} \mathrm{was} \mathrm{noticed} \mathrm{this} \mathrm{time.} \mathrm{The} \mathrm{extra} \mathrm{length}$ of fibers from the other three ends were then removed by cutting them off with a sharp blade.

\section{DESCRIPTION OP PURIFICATION LOOP FOR VARIOUS MODES IN SMALL-SCALE OPERATION}

A photograph of the experimental setup used in this work is shown in Figure 3-8. The setup consisted mainly of three sections: feed line, sweep or permeate line and a membrane liquid supply line all tied to the HFCLM permeator. The permeator was maintained at $25^{\circ} \mathrm{C}$ by completely immersing it in a constant temperature water bath (CTB). The water circulation and temperature control were assured by a constant temperature circulator (Haake Inc., Saddlebrook, NJ; model NK 22). Only the sweep section was modified whereas the other two sections essentially remained unaltered in different modes of operation. All pieces of equipment in the setup were interconnected with either 1/4-inch stainless steel tube or 1/4-inch thickwalled polypropylene tubing. A primary standard gas mixture cylinder (Matheson, East Rutherford, NJ) containing $\mathrm{CO}_{2}$ and $\mathrm{N}_{2}$ or $\mathrm{CO}_{2}$ and $\mathrm{CH}_{4}$ was used as feed gas and pure helium or nitrogen was used as sweep gas. Shell liquid was pressurized by pure helium gas in all experiments.

\section{SWEEP GAS MODE}

The setup is described schematically in Figure 3-9. Feed line consisted of an oil filter, OF (one foot long, $1 / 2$ inch stainless steel pipe, packed with glass wool), a feed humidifier, FH (a 20 liter stainless steel vessel, Millipore Corporation, Bedford, MA; equipped with a small pressure gauge of range, 


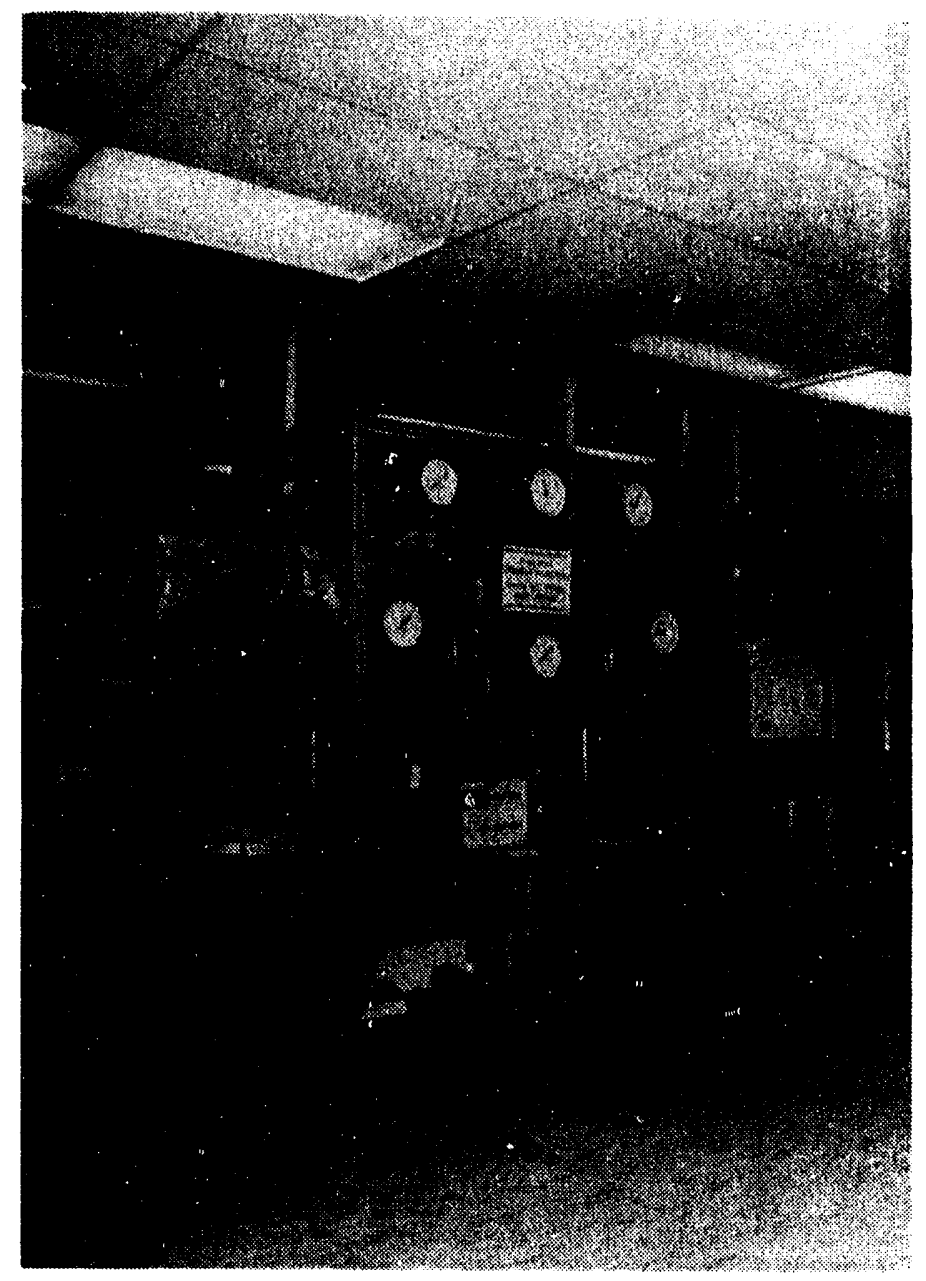

FIGURE 3-8

PHOTOGRAPH OF THE PURIEICATION LOOP WITH THE PERMEATOR IMMERSED IN THE CONSTANT TEMPERATURE BATH 

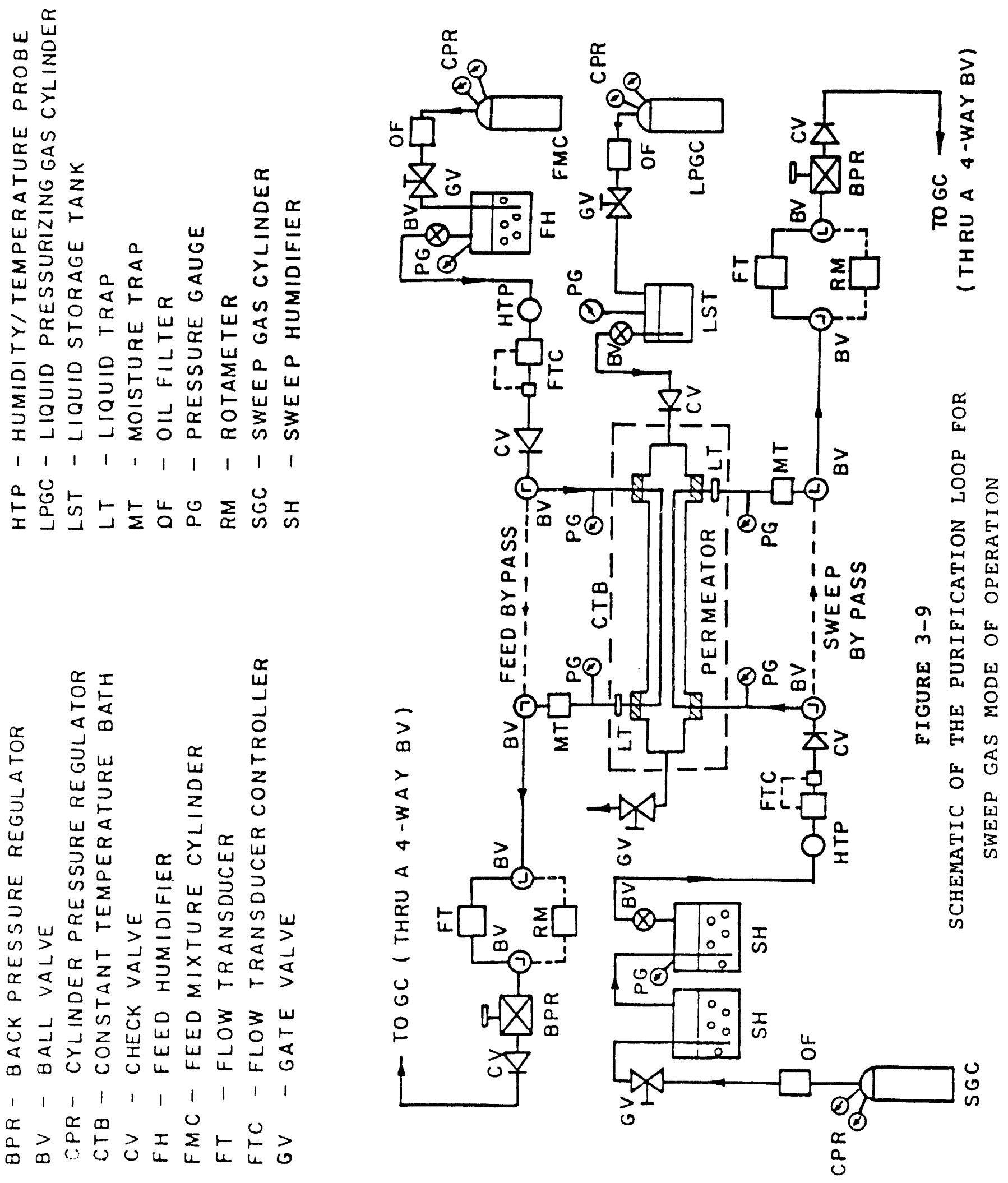
1) - 160 psig and a satety valve), a humidity-temperature probe, HTP (Vaisala Inc., Woburn, MA; model HMP 32UT), an electronic mass flow transducer controller, FTC (Matheson model 8141-0452; lange, 0 - $500 \mathrm{sccm}$ ), a Heise pressure gauge (Dresser Industries, Newton, CT; range, $0-250$ psig). The humidity and the temperature of the feed gas stream coming out of $\mathrm{FH}$ were next monitored by HTP which was mounted in a stainless steel cylindrical cell connected to the gas line. The flow rate of the humidified feed gas was controlled and measured by FTC. A check valve, CV, was provided to prevent accidental injection of liquid from the permeator into the electronic flow controller. A three-way ball valve, located after the check valve, enabled the feed stream to bypass the permeator.

The purified feed gas stream from the permeator was passed successively through a liquid trap, LT, a pressure gauge (Matheson; range, 0 - 200 psig), a moisture trap column, MT, and a three-way bypass ball valve. The liquid trap was nothing but a three-inch-long $1 / 2$-inch brass pipe. It was essentially a vertically located tee with the gas coming in at the branch line and going out through the top while liquid droplets collected at the closed bottom end. This device prevented carryover of the liquid droplets which appeared at the permeator tube sheet (from condensation or fiber leakage through the damaged fibers of the permeator, or both).

Moisture from the purified feed gas stream was eliminated by a moisture trap MT (Drierite Column Model \#L68GP; W. A. Hammond Drierite Co., Xenia, OH), prior to the measurement of the flow rate by either a Fisher and Porter rotameter, RM (Phillips Co., South Hackensack, NJ; model $10 \mathrm{~A} 3555 \mathrm{AY}$ ) or by a Matheson electronic mass flow transducer (model 8141 - 0452 ; range, 0 - $500 \mathrm{sccm}$ ) connected in parallel. However, only the transducer was used for accurate measurement of the purified feed gas flow rate. The gas stream was next channelled respectively through a Fairchild back pressure regulator, BPR (Epec Sales. Towaco, NJ; range, 2-150 psi) to control the pressure of the feed gas stream and a check valve.

The composition of the purified feed gas stream emerging from the check valve was measured periodically by a gas chromatograph. A four-way switching ball $\because$ alve was utilized to this end beyond the check valve. 
The nature of the instrumentation in the sweep gas line was essentially identical to that in the feed gas line with the following exceptions. To accommorate higher sweep gas flow rate, two 20 liter Millipore stainless steel vessels, SH, were connected in series for humidification of the sweep gas stream. Moreover, an electronic mass flow transducer and transducer - controller, FTC, of higher flow rate ranges (Matheson model 8141 - 0413; range, $0-1000 \mathrm{sccm}$ ) were used to measure and control the sweep side gas flow rate. A Matheson test gauge (range, $0-100$ psig) and a Heise compound gauge (range, 30 inch vac - 15 psig) were used to measure the pressure at the sweep gas inlet and outlet locations of the permeator, respectively. The Fairchild back pressure regulator, used to control the sweep stream pressure, had a range of $0-10$ psi.

The permeator shell side inlet was connected to the membrane liquid storage tank, LST (a 10 liter Millipore stainless steel vessel), through a check valve and an on-off ball valve. The liquid storage tank was pressurized by a gas cylinder, LPGC, through an oil filter and a gate valve. The pressure of the liquid line connecting LST to the permeator was indicated by a Matheson test gauge (range, $0-200 \mathrm{psig}$ ). This pressure was always maintained at a level higher than those of both the feed and the sweep gas streams. The pressure of the LST could be independently changed if necessary, by manipulating the on-off ball valve. This was particularly useful during start-up and shutdown of the system. The shell side outlet was kept closed by a gate valve. It was opened to drain the membrane liquid from the permeator when the system was shut down and the permeator was not operational.

The gas flow rate readings of the four transducers were monitored centrally by a Matheson digital readout and control module (model 82.49 ). The same module was used to set and control the gas flow rates at the permeator inlets. The humidity and temperature of both gas streams entering the permeator were displayed on a digital indicator (Vaisala Inc., Woburn, MA; model HMI 32). The indicator utilized a HMS 13 probe selector to measure the humidity and temperature of one gas stream at a time.

For purification runs with $40 \% \mathrm{CO}_{2}-60 \% \mathrm{CH}_{4}$ feed gas mixtures, additional steps 
were taken. The complete purification loop was enclosed with a polyethylene cover with an opening at the lop which was connected to the exhaust hood. Inside this enclosed space was located a $\mathrm{CH}_{4}$ detector (Model 462484, MSA Inc., Pitts., PA). Outside the cover, a similar detector was located. Both of them were connected to a control module (Model 465740, MSA Inc., Pitts., PA) and horn outside the cover. Additionally, a $N_{2}$ cylinder was provided to flush the feed gas line after a separation run was carried out.

\section{CONVENTIONAL POLYMERIC MEMBRANE MODE}

No modification of the purification loop was needed to operate the system in the conventional polymeric membrane mode. In the absence of a sweep gas, one end of the permeator (previously sweep inlet end) was kept closed; the threeway ball valve in the sweep gas inlet line was switched to the other direction so that it automatically closed that end. The schematic of the corresponding experimental loop is shown in Figure 3-10.

\section{VACUUM MODE}

To carry out purification studies in the vacuum mode, a few modifications were made. The modified schematic of the same is shown in Figure 3-11. Note that, no change was made in the feed gas and membrane liquid supply lines. In the absence of a sweep gas, one end of the permeator (previously inlet end) was kept =losed. The pressure of the closed end was measured by a Heise vacuum gauge. A Heise compound gauge (range 30 inch vac - 15 psig) was used to monitor the pressure at the permeate outlet through which vacuum had been pulled. Duo-seal two-stage lotary vane vacuum pump (Model \#1400, Sargent-Welch Scientific Co., Skokie, IL) was used to create and maintain the vacuum. Moisture from the permeate $s t r e a m$ was eliminated by a Drierite Column Model \# L68cip (w. A. Hammond Drierite Co., Xenia, $O H$ ) before the vacuum pump. An oil filter, packed with glass wool and Drierite, was provided after the vacuum pump exhaust line to eliminate the oil and moisture from the permeate stream. The permeate line was connected through a four-way switching ball valve to the gas chromatograph (GC) to measure the composition of the permeate stream periodically. The permeate flow rate was measuled by a soap bubble flowmeter atter the $r(c$. 

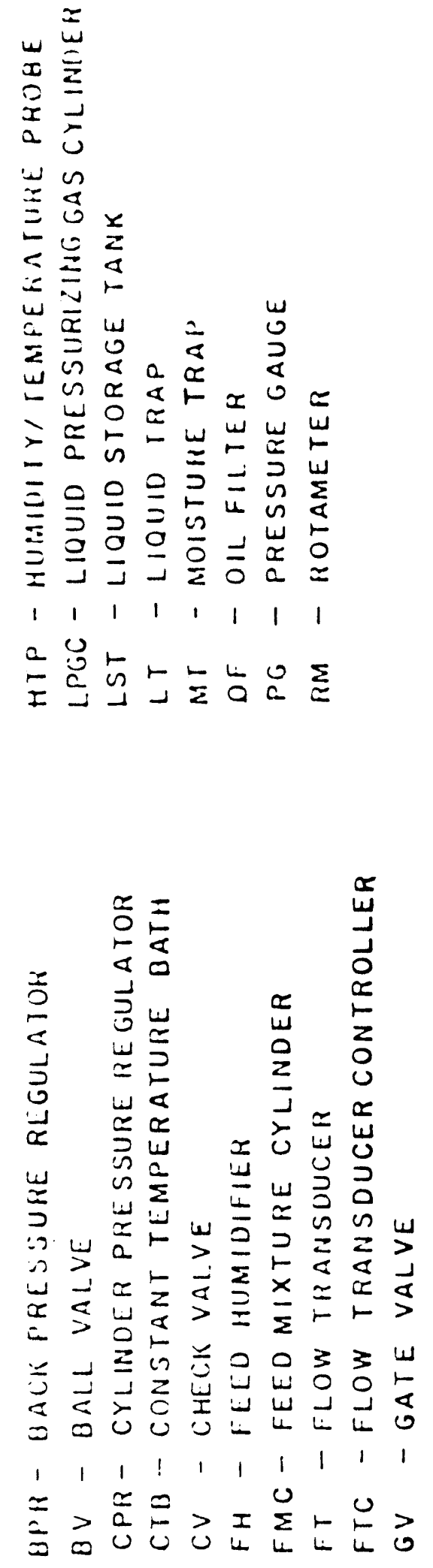

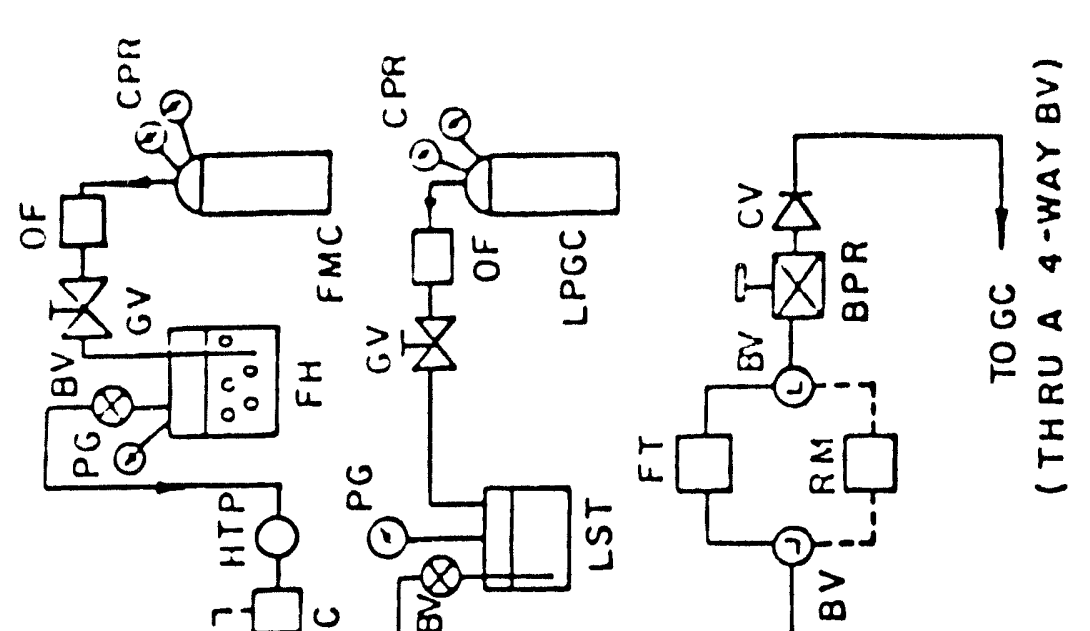

2

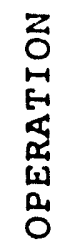

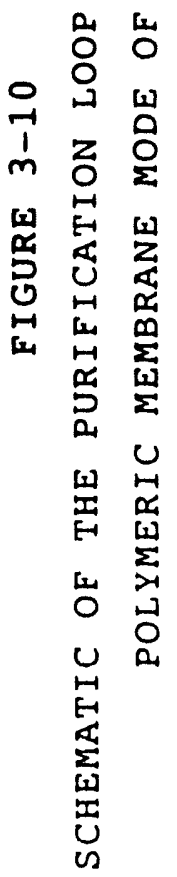



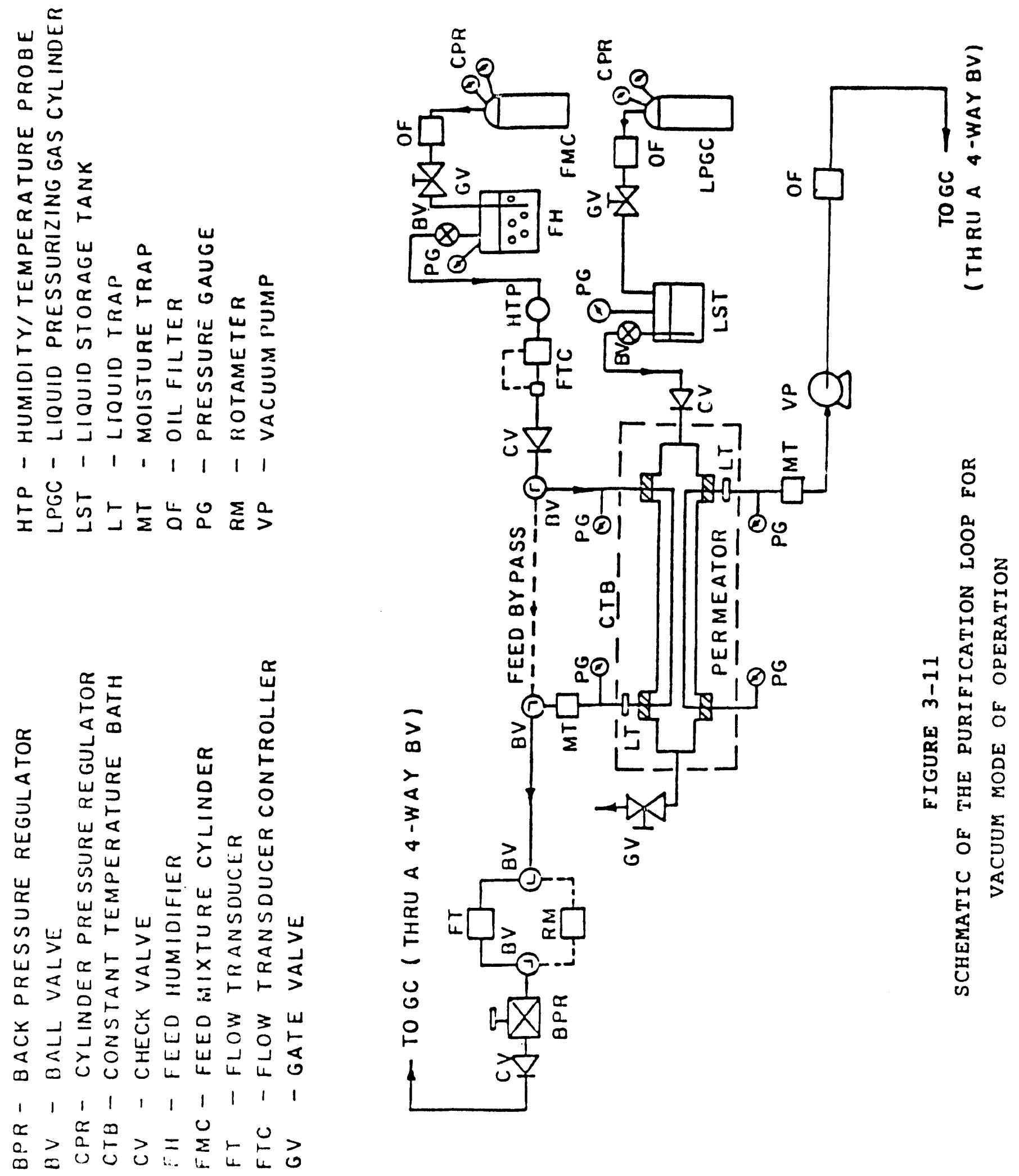
For purification runs using $\mathrm{CO}_{2}-\mathrm{CH}_{4}$ gas mixtures as feed, an explosion-proof single-stage vacuum pump (Model \#1410W-01, Sargent-Welch Scientific Co., Skokie, IL) was used. The permeate outlet gas at the vacuum iump outlei was allowed to flow through the oil filter-cum-Drierite colura to the four-way switching valve and then it was sent either to the GC or to the exhaust hood. Permieate gas fllow rate was measured by a soap bubble flowmeter kept under the exhaust hood. The outlet from GC was also connected to the exhaust hood. At the end of an experiment, feed gas was switched to ni ogen gas to flush both feed line as well as the permeate line.

\section{SWEEP WATER MODE}

Purification studies in the sweep water mode required some modifications. No change was made in the feeri gas and the membrane liquid supply line. Two large (80 liter) plastic vessels were used to store distilled water. A Cole Parmer Gear Pump (Model \#7144-04) with a Variable Speed Controller (range, 165 - 3600 rpm) was used to circulate distilled water through the sweep fibers. Pressures at the sweep water inlet and outlet ends were measured by $2-1 / 2$-inch-diameter Matheson pressure gauges (range: $0-60$ psig). The modified schematic of the loop is shown in Figure 3-12.

\section{DESCRIPTION OF THE LARGER PURIPICATION LOOP}

The experimental setup used for long-term purification of model biogas mixture is described schematically in Figure 3-13. The instrumentations in the setup were supported on a laboratory flexframe structure. The interconnections between various equipment were either by $1 / 4$-inch stainless steel tube or by 1/4-inch thick-walled polypropylene tubing.

Although the plumbing in this purification loop was more complex than the one we described earlier, it essentially contained three different flow lines, e.g., feed gas line, sweep gas line and membrane liquid storage line. Three $\mathrm{CO}_{2}$ gas cylinders and three $\mathrm{CH}_{4}$ (or $\mathrm{N}_{2}$ ) cylinders, connected by two separate manifolds ensured a constant supply of gases. Each gas stream was sent through an oil filter (1/2-inch stainless steel pipe, packed with glass wool) to remove 

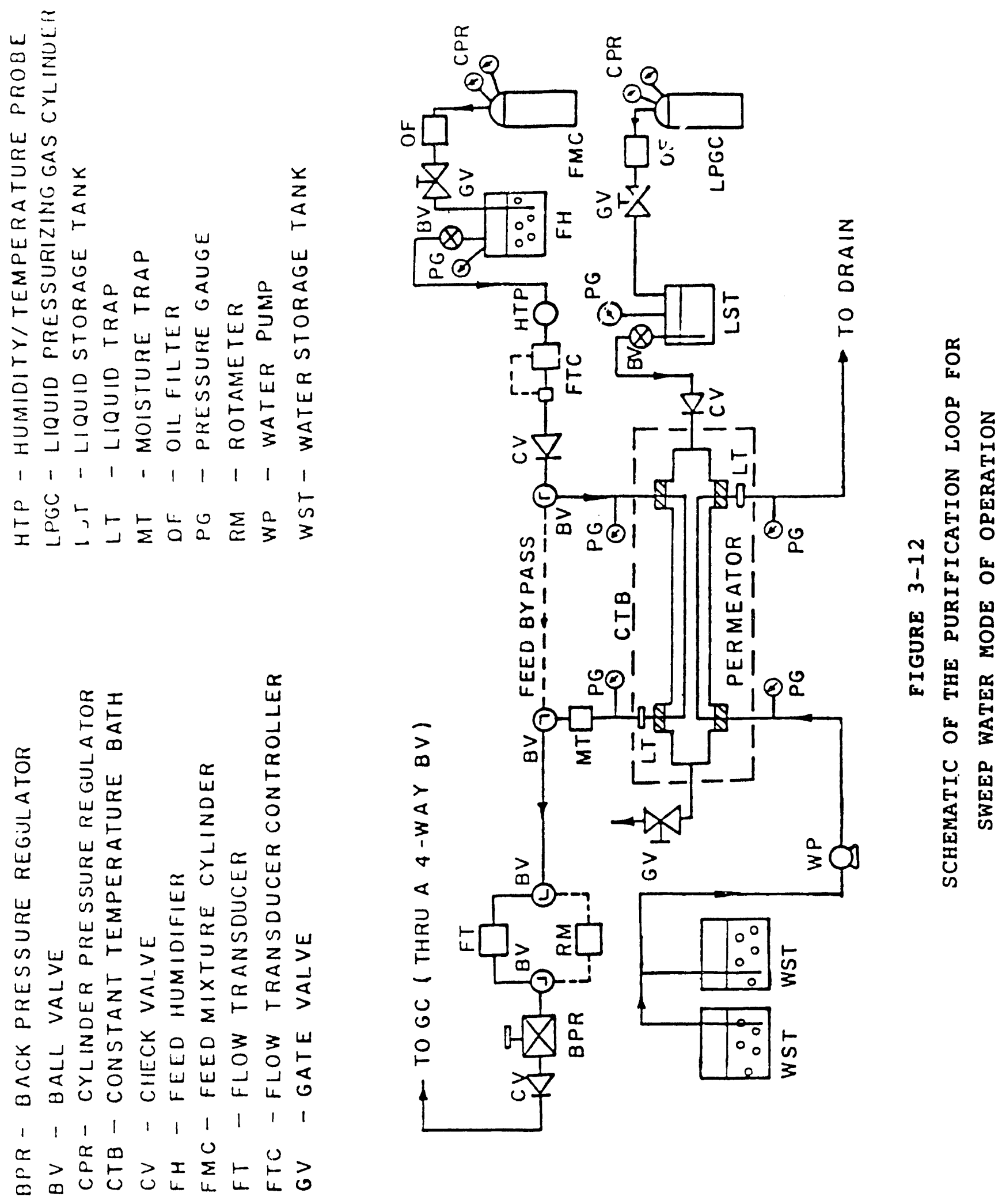


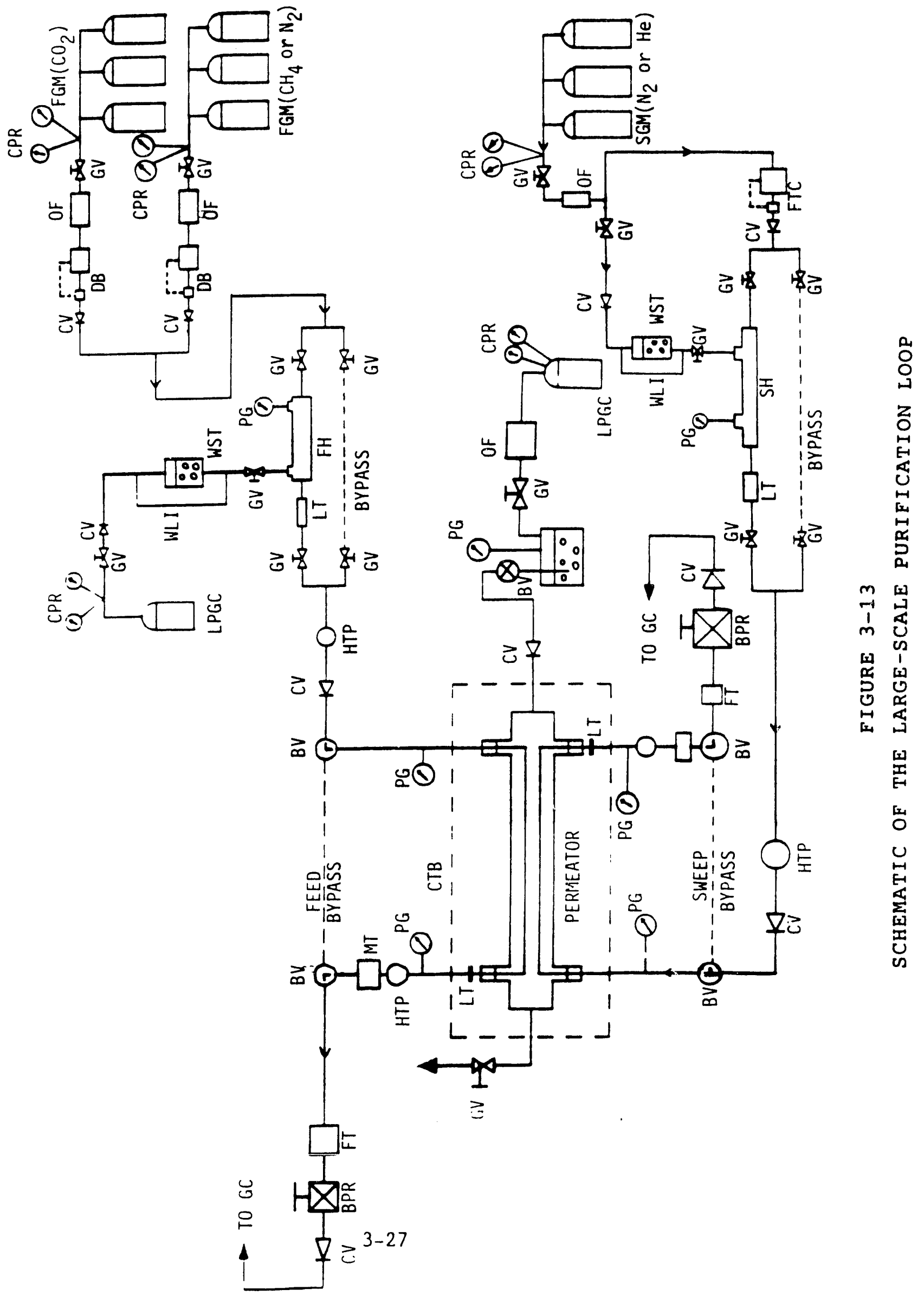


oil droplets, if any. The filtered gas streams were connected to two electronic flow transducer - controllers (transducer model no. 8102; controller model no. 8202) of a Matheson Dyna-blender (Model No. 8250). This blender allowed one to prepare a gas mixture of a specific composition at a given flow rate. Thus, model biogas feed mixture was prepared in situ by blending $\mathrm{CO}_{2}$ and $\mathrm{CH}_{4}$ (or $\mathrm{N}_{2}$ ) gases.

The blended gas mixture was sent through a feed humidifier, a 21-inch-long hollow-fiber membrane contactor, made in our laboratory for this particular project. It contained 6000 Celgard polypropylene hollow fibers. The structure of this humidifier was similar to that of a shell-and-tube type heat exchanger. The feed gas was passed through the tube-side and the shell was completely filled with water. To ensure constant supply of water, a water storage tank was attached to the shell-side of the humidifier. The pressure of the water tank was maintained at a higher level than that of the feed gas pressure by a gas cylinder, connected to the other end of the tank. An indicator was also provided to identify the water level in the storage tank. The trap located after the humidifier captured water droplets which appeared due to condensation and/or leakage through the damaged fibers. The humidifier bypass line allowed one to switch instantly from humidified gas mixture to partially humidified or completely dry gas mixture.

The humidity and the temperature of the feed gas stream were monitored by a probe (model 32UT, Vaisala Inc., Woburn, MA), mounted in a Teflon cell connected to the gas line. The check valve prevented any accidential flow in the opposite direction. A three-way ball valve enabled the feed stream to bypass the permeator. The feed stream pressure at the permeator inlet was indicated by a pressure gauge (0-200 psig, Matheson, E. Rutherford, NJ). The purified gas stream from the permeator was passed through a liquid trap. A pressure gauge indicated the outlet gas pressure. The humidity and temperature of the purified gas stream were measured by another humidity probe. Moisture from the purified gas stream was eliminated by a Drierite column (W.A. Hammond Drierite Co., Xenia, $\mathrm{OH}$ ) prior to the measurement of its flow rate. The flow rate was measured by an electronic mass flow-transducer (model No. 8202; Matheson). The gas stream was then passed through a Fairchild back pressure regulator (Epec Sales, Towaco, $N J$ ) and a check valve. the back pressure 
regulator was used to control the pressure of the feed gas stream. The composition of the purified gas stream was measured periodically by a gas chromatograph.

The instrumentations in the sweep gas line were essentially similar to those of the feed gas line with the following exceptions. A pure gas $\left(\mathrm{N}_{2}\right.$ or $\left.\mathrm{He}\right)$ was introduced as a sweep gas, therefore, only one gas ranifold containing three cylinders was used. The sweep line water storage tank for humidification was pressurized by the sweep gas itself; no separate gas cylinder was necessary here.

The permeator shell-side inlet was connected to a membrane liquid storage tank through a check valve and an on-off ball valve. The liquid storage tank was pressurized by a gas cylinder through an oil filter and a gate valve. The pressure of this liquid line was indicated by a Matheson test gauge. This pressure was always maintained at a level higher than those of both the feed and sweep gas streams. The shell-side outlet was kept closed by a gate valve. This particular valve was used to drain the membrane liquid from the permeator.

The permeator shell was maintained at a desired temperature level by completely immersing it in a water bath. It was made of galvanized iron with insulations on all five sides. The inner surface of the bath was painted with a special polyurethane and epoxy to minimize corrosion by water. The water temperature was maintained by a constant temperature circulator (Haake Inc., Saddle Brook, NJ, model NK 22).

The gas flow rate readings of the transducers were monitored centrally by a Matheson digital readout and control module (model 8209). The humidity and temperature of all gas streams were displayed on a digital indicator (Vaisala Inc., Woburn, MA; model HMI 32). The indicator utilized a HMS 13 probe selector to measure the humidity and temperature of one gas stream at a time. A photograph of the experimental setup is shown in Figure 3-14.

\section{EXPRRIMENTAL PROCEDURE}

In both cases, the entire flow loop was occasionally tested for leak by 


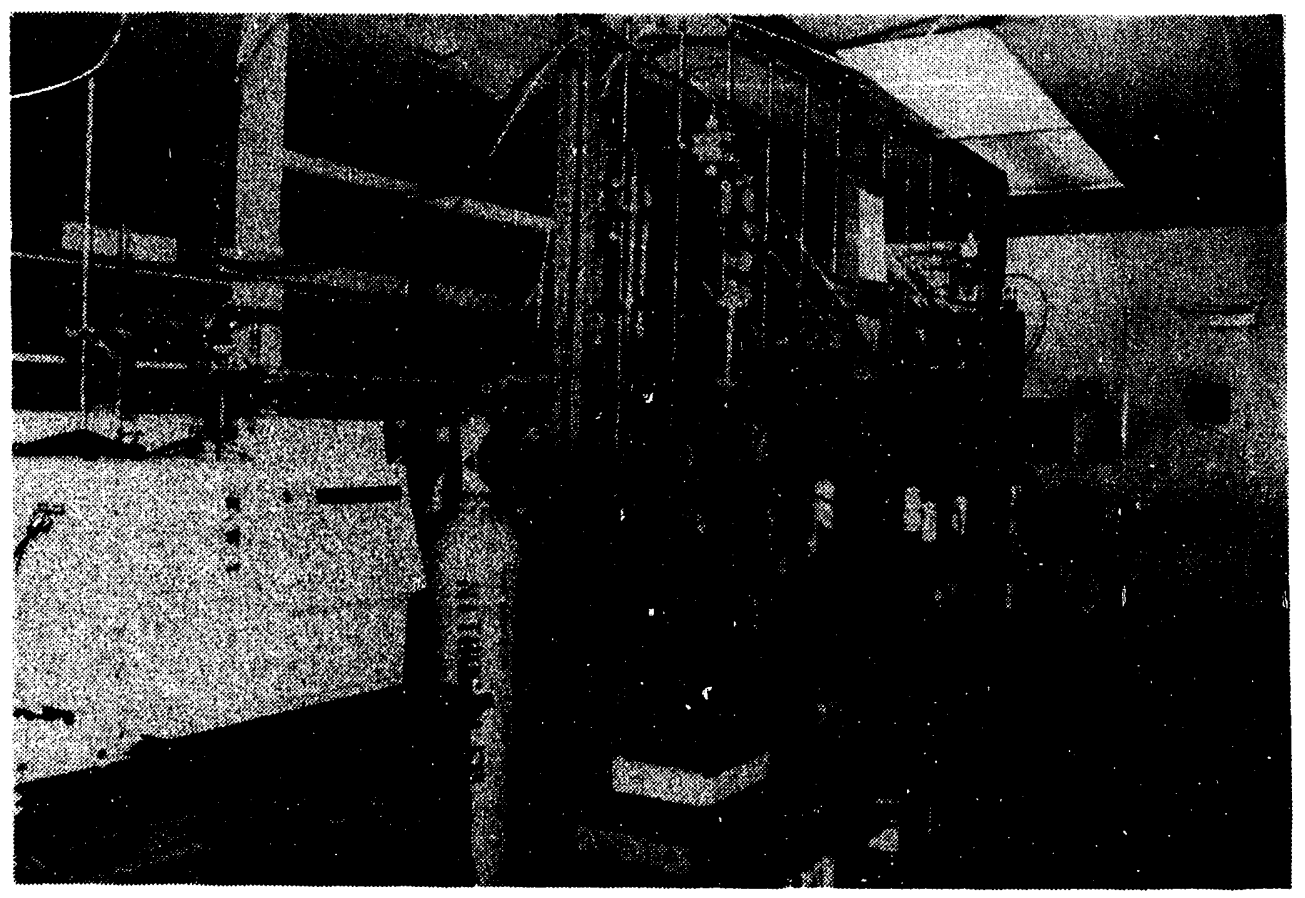

FIGURE 3-14

PHOTOGRAPH OF THE LARGE-SCALE PURIFICATION LOOP 
pressurizing the system with $\mathrm{N}_{2}$ gas. Dry gas was used in all the experiments where water acted as liquid membrane. Whenever aqueous solution was used as membrane, humidified feed gas was used. For sweep gas mode of operation with such membranes, sweep gas was also humidified. However, dry feed gas was used in the sweep water mode experiments.

To start the system, the permeator shell side was first filled with the membrane liquid by manipulating the on/off valve, located on the liquid storage tank. The latter was initially maintained at about 10 to $15 \mathrm{psig}$. The feed and sweep gas flow were then initiated, keeping the pressure in the feed and the sweep gas line lower than the storage liquid pressure. A helium cylinder was always used to pressurize the membrane storage tank. The pressure in either flow line was determined primarily by the back pressure regulator setting. The membrane liquid storage tank pressure was also increased slowly to the desired level. The feed and sweep gas flow rates were then set at the desired values. Finally the feed inlet and sweep outlet pressures were set at particular values by manipulating the back pressure regulators. Measurements of the gas flow rates, pressures, humidities, temperatures and outlet gas compositions were made at regular interval.

To shut down the system, the valve connecting the shell liquid storage tank with the permeator was first turned off and the gas pressure as well as the flow rates in each line (sweep gas mode) were slowly reduced. The liquid pressure in the permeator shell was released by opening the shell side gate valve.

The experimental procedure under conventional polymeric membrane mode was similar to that of sweep gas mode with the exception that no sweep gas was used. One end of the permeator (previously sweep inlet end) was closed by switching the three-way ball valve. Thus the permeated gas was only flowing through the permeate fibers countercurrent to the feed gas. Permeate flowrate was also checked by the soap-bubble flow meter in all the experiments.

All experiments under vacuum mode of operation needed thorough leak testing of the lines under vacuum. The vacuum pump was turned on and the valve between the inlet of vacuum pump and the Drierite column was kept open. Once the desired 
vacuum was achieved, the valve was closed and the system was kept in that condition for about two hours to check if there was any drop in vacuum. The experiment was started when the system was found to be leak proof.

The pressurization step was similar to that of sweep gas mode of operation, but the vacuum was applied to the system while the shell liquid pressure was at about 15 psig. Gradually, both shell liquid pressure and the feed side gas pressure were raised to the desired level with proper setting of feed gas flow rate. Measurement procedures were similar. Shut down procedure was also similar but at first the vacuum pump was disconnected from the system. However, in the case of $\mathrm{CO}_{2}-\mathrm{CH}_{4}$ gas mixture as feed, experiment was continued by switching the feed gas to $\mathrm{N}_{2}$ and continuously the feed and the permeate streams were monitored by $\mathrm{GC}$ for the presence of $\mathrm{CH}_{4}$ till there was essentially no $\mathrm{CH}_{4}$ in either stream.

When the system was operated in sweep water mode, the procedure undertaken was exactly similar to those used in the case of sweep gas mode of operation. However, the flow of sweep water was started when shell liquid pressure as well as feed side pressure and flow rate were set. The flow rate of water was measured at the outlet by a measuring cylinder and stop watch.

\section{MEASUREMENT OP GAS PERMEABILITY THROUGB THE LIQUID MEMBRANE}

The measurement of permeability of $\mathrm{CH}_{4}$ through water was carried out using the immobilized liquid membrane (ILM) technique. The same technique was adopted to measure the permeabilities of $\mathrm{N}_{2}$ and $\mathrm{CO}_{2}$ through aqueous solution of 20 wt\% DEA at different partial pressures of $\mathrm{CO}_{2}$.

Permeation rate of any gas species through a liquid membrane was delermined by the sweep gas method (Bhave and Sirkar, 1986). Feed gas (pure or a mixture) was first completely humiditied and passed over a film containing water or aqueous soll1ilill ill lli, leat cell at a given pressure (usllilly fileater than atmospheric hut not nereisalli, on the othes side of the $111 \mathrm{~m}$, a completely humilfied helium swerp gas streall is passed at essentially atmospheric:

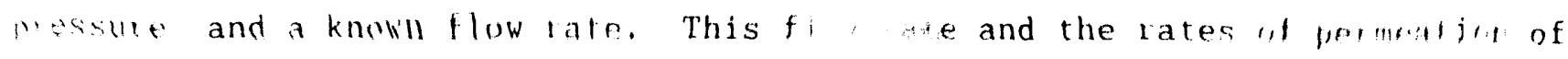
the ompononm of the gas mixture were such that the bermo ad perit. ... not 
present in the sweep gas at concentrations higher than, say 2-3 mole percent. This sweep gas stream was then analyzed by a gas chromatograph. The permeation rate was obtained easily; knowing the sweep helium flow rate and the permeated gas species content of this stream is equivalent to knowing the flow rate of that species, the latter coming about strictly due to permeation.

The available experimental setup for the measurement of gas permeability through an aqueous liquid membrane immobilized in a hydrophobic microporous film is shown in Figure 3-15. The setup and the procedure are described below.

Pure water or the aqueous solution was immobilized in the pores of a hydrophobic microporous polypropylene film Celgard $2400\left(2.54 \times 10^{-3} \mathrm{~cm}\right.$ thick with a porosity of 0.38 and average effective pore size of 0.02 micron) by the exchange technique of Bhave and Sirkar (1986). The exchanged Celgard 2400 film with a nominal diameter of $5.1 \mathrm{~cm}$ was placed in the test cell. The Celgard film was supported by two thick Accurel polypropylene films (each $0.010 \mathrm{~cm}$ thick and $3.5 \mathrm{~cm}$ in diameter having an average pore size of 0.2 microns from Armak, Chicago, Illinois) and a porous, 6-mil-thick, fine circular stainless steel screen having the same nominal diameter as Accurel films (Pall Trinity Micro Corporation, Cortland, NY). The membrane was sealed by a set of 0 -rings measuring $4.4 \mathrm{~cm}$ and $5.7 \mathrm{~cm}$. To be especially noted here is that the pore size of Accurel is one order of magnitude larger than the pore size of Celgard. Thus, Accurel films were used simply as a backing to protect the Celgard film from any accidental sharp edges of the stainless steel screen employed to support the high pressure difference across the ILM. The area available for gas permeation was $13.19 \mathrm{~cm}^{2}$. The test cell was immersed in a constant temperature bath. (HAAKE constant temperature immersion circulator, Model D1) obtained from Fisher Scientific, Springfield, N.J.

The placing of this film in the test cell (TC) was preceded by adjustment of the desired flow rates of humidified streams of feed gas and the sweep helium gas, respectively. This was done with the help of Matheson Digital transducers and control valves FTC-s shown in Figure 3-15. In order to ensure complete humidification of both the feed and sweep gas streams before they enter the test cell, the humidification process was carried out for each stream for at least 12 hours in two high-pressure Hoke sample cylinders before the start of 


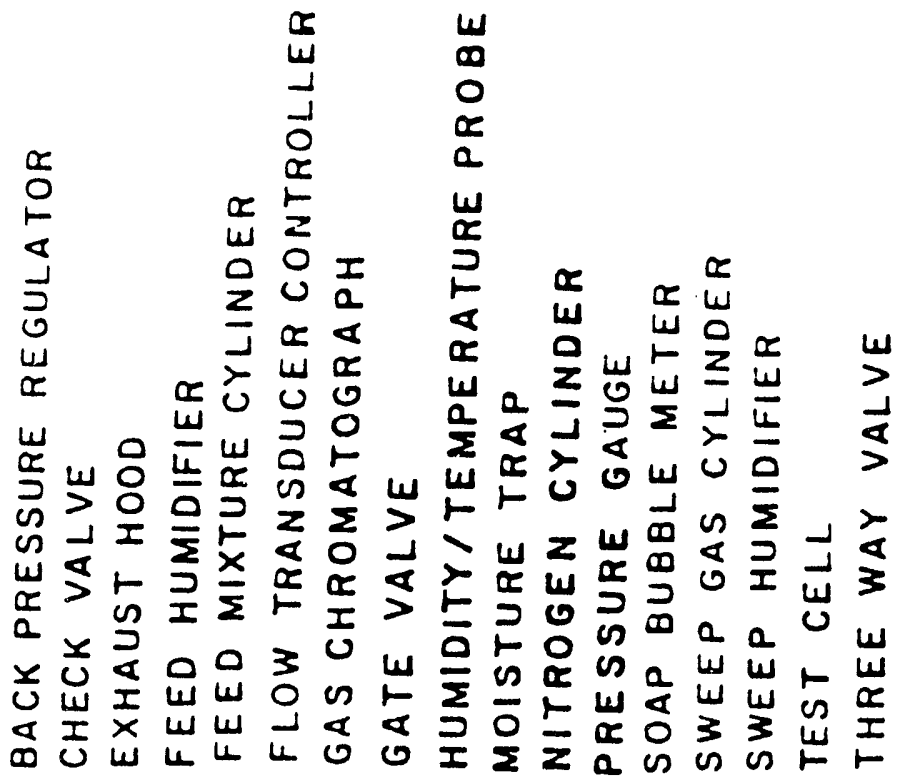

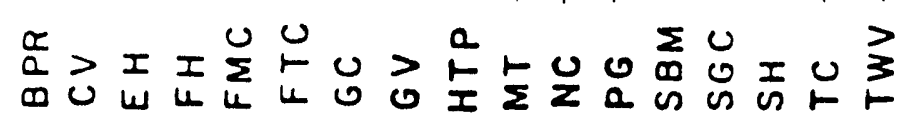

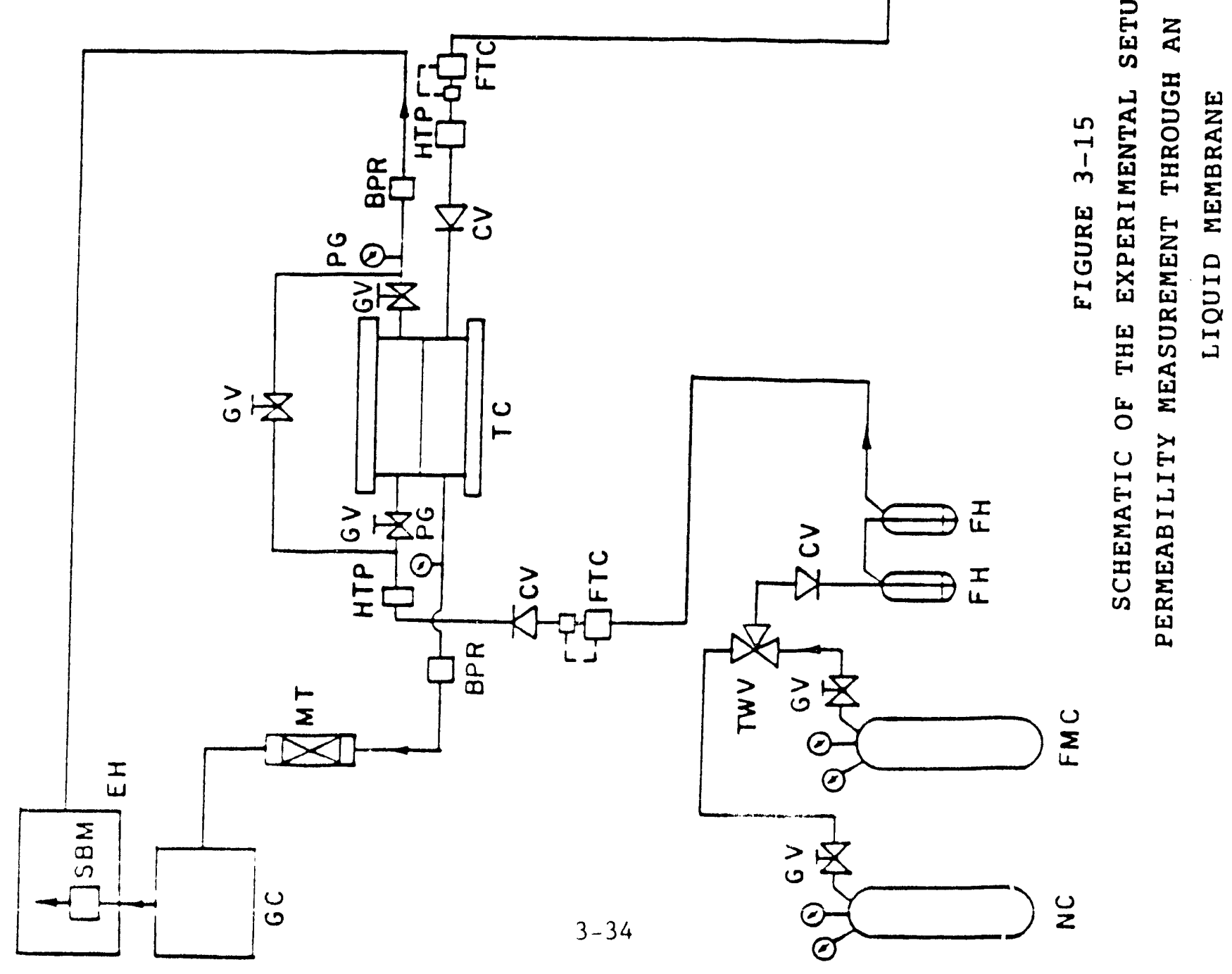


each permeation experiment. Each humidifier vessel was filled with about 400 cc of distilled water. Additional water was added from time to time to compensate for the loss during humidification of the gas streams. The gas space volume above water in each humidifier ( $\mathrm{FH}-\mathrm{s}, \mathrm{SH}-\mathrm{s}$ ) was around $100 \mathrm{cc}$. With about 10-15 cc/min flow rate of either the feed stream or the sweep stream, the humidified gas in two successive reservoirs was sufficient to ensure an uninterrupted supply of completely humidified gas for a long time.

Further, in order to verify the extent of saturation of the sweep gas and the feed gas stream with respect to water vapor, the probes (HTP) of a high precision digital relative humidity/temperature measurement system (Model HS-2CHDT-2A, Thunder Scientific Corporation, Albuquerque, New Mexico) were located just before the cell inlets. The desired feed side pressure was next achieved by means of the back pressure regulator BPR. The sweep gas was always maintained at around 0.1 psig to prevent any possibility of surrounding air leaking into the system. The sweep gas samples were drawn into the Gas Chromatograph column through a gas sampling valve for analysis. Samples were drawn over regular intervals (e. g., 15-20 minutes) to determine the progress of the permeation experiment. To eliminate moisture in the sweep gas so that there is no long tailing in the TCD output, the sweep gas sample was passed through a moisture trap (MT) (essentially a column of Drierite) in the sweep line just before the gas sampling valve in the GC.

Initially, leak testing was carried out on the entire loop using nitrogen from a nitrogen cylinder (NC). The same nitrogen cylinder was also kept as standby for flushing the complete flow loop when a mixture containing $\mathrm{CH}_{4}$ was utilized.

Since the flow rate of the sweep gas is quite important for the accurate determination of the permeability of any gas species, the sweep flow line after the GC was taken to an exhaust hood where the flow rate was measured using a soap bubble flow meter. The feed gas line was also connected to the exhaust hood after the back pressure regulator.

\section{CALIBRATION AND ANALYSIS}

The compositions of the purified feed gas stream and the sweep or permeate 
outlet stream were measured by a Varian Gas Chromatograph (GC) (Varian Instruments, Palo Alto, CA; ) with a thermal conductivity detector (TCD). Varian model $3700 \mathrm{GC}$ was used in small-scale operation whereas model $3400 \mathrm{GC}$ was used in large-scale operation. The response from the detector was recorded by a digital reportins integrator (Hewlett Packard, Paramus, NJ; medel 3390 A or Varian model 4290). A six-feet-long $1 / 8$-inch stainless steel Porapak $\mathrm{N} 80 / 100$ mesh columns was used for separation of the gas mixture.

Since helium was used as a carrier gas, the thermal conductivity detector could not detect He. Feed and sweep outlet streams would contain $\mathrm{CO}_{2}, \mathrm{~N}_{2}$ and helium in varying proportions when helium was used as sweep gas and gas mixture containing $\mathrm{CO}_{2}$ and $\mathrm{N}_{2}$ was used as feed. Therefore, it was necessary to develop calibrations in terms of absolute areas of $\mathrm{CO}_{2}$ and $\mathrm{N}_{2}$ rather than area percent. The helium content in the samples could then be estimated by subtracting the combined $\mathrm{CO}_{2}-\mathrm{N}_{2}$ percentage from 100. The calibration curves were developed for $\mathrm{CO}_{2}, \quad \mathrm{CH}_{4}$ and $\mathrm{N}_{2}$ by utilizing primary standard gas mixtures and/or gas mixtures obtained by carefully controlled mixing of pure gases in different proportions using a modular Dyna-blender (Matheson, model 8250). A few sample calibration curves for Varian 3700 and $3400 \mathrm{GC}$ are shown in Figures 3-16 and 3-17, respectively. 


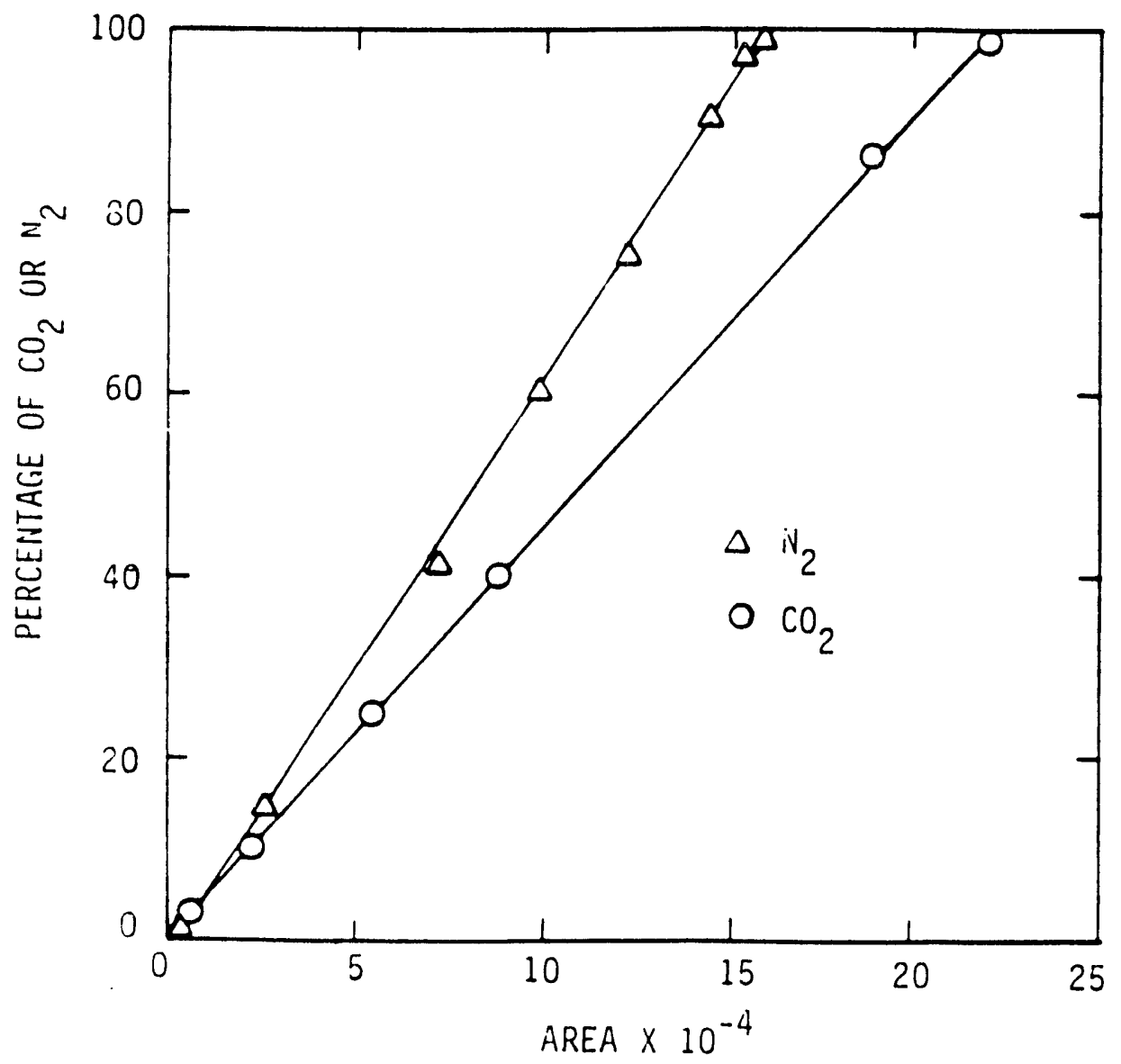

FIGURE 3-16

ABSOLUTE AREA CALIBRATION FOR $\mathrm{CO}_{2}$ AND $\mathrm{N}_{2}$ FOR VARIAN $3700 \mathrm{GC}$ 


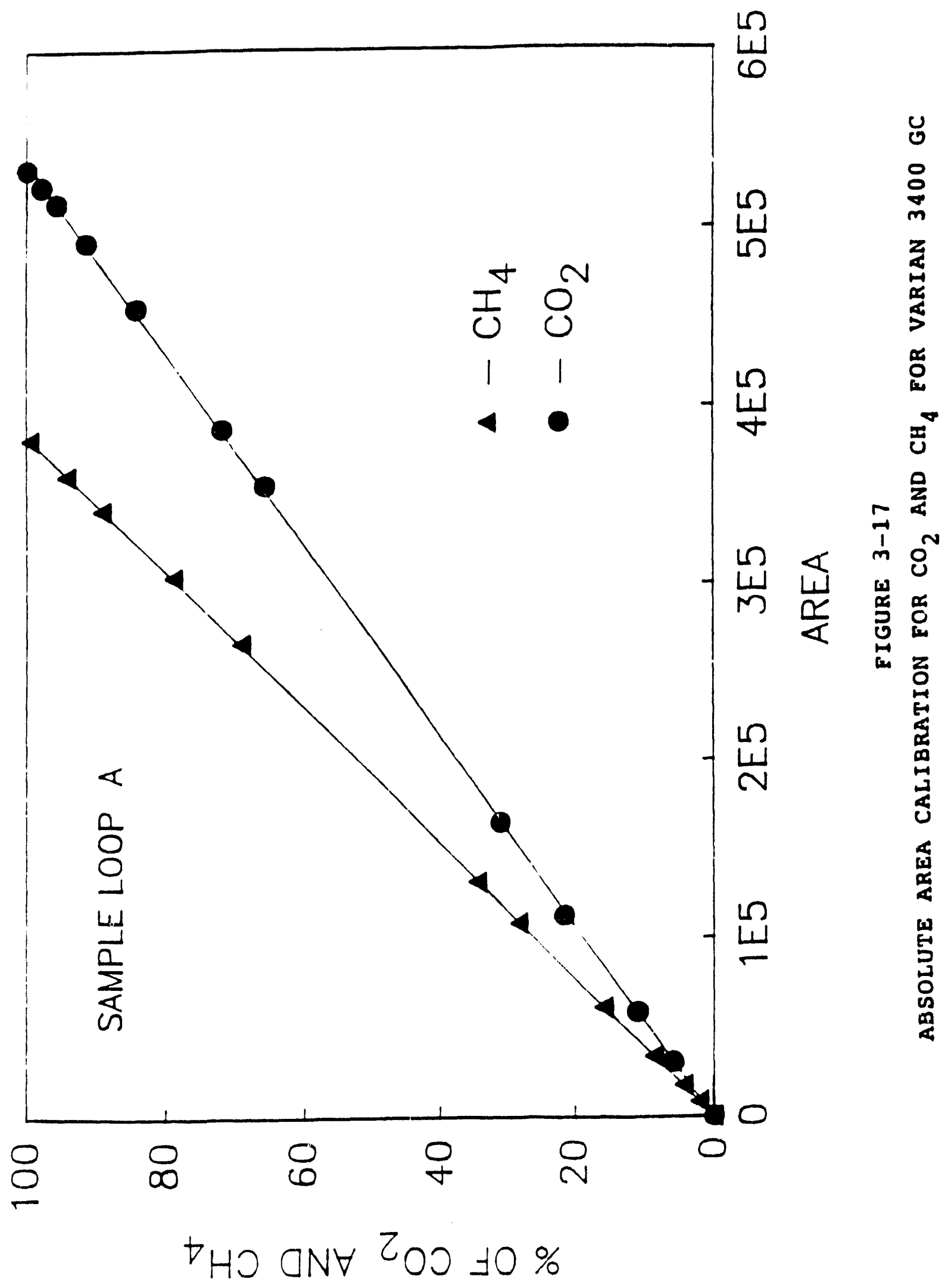




\section{Section 4}

\section{RESULTS AND DISCUSSION}

Experimental data and numerical simulation results for biogas purification in HFCLM permeators are presented in this section. Results of each mode of operation (e.g., sweep gas, conventional, vacuum and sweep water) are discussed separately. The feed mixture compositions and other parameters, used in a given set of experimental studies, are clearly mentioned in each table.

\section{ESTIMATION OF PURE COMPONENT GAS PERMEABILITIES THROUGH VATER}

The permeability of a gas species, $Q_{i}$, through a pure water film may be estimated fron. the product of the diffusivity of the gas species through water, $D_{i w}$, and the solubility of the gas species in water, $H_{i w}$ :

$$
Q_{i}=D_{i w} \times H_{i w}
$$

The diffusivity and the solubility values were obtained from a variety of sources cited in Table 4-1. Since all the experiments were carried out at $25^{\circ} \mathrm{C}$, the permeability values of $\mathrm{CC}_{2}, \mathrm{~N}_{2}$, He and $\mathrm{CH}_{4}$, reported in Table 4-1, were used for all the numerical simulations where water was used as liquid membrane.

Permeability of $\mathrm{CH}_{4}$ was also determined experimentally by measuring the permeation rate of pure $\mathrm{CH}_{4}$ through fully exchanged Celgard 2400 ILM film containing water as immobilized liquid membrise. The results, presented in Table 4-2, show that the permeability of $\mathrm{CH}_{4}$ through ure water is more or less constant over the experimental range of pressure differences. These values are quite close to that obtained from the literature (Table 4-1).

\section{ESTIMATION OF PURE COMPONENT GAS PERMRABILITIES THROUGH} AQUEOUS SOLUTIONS OP DEA

Permeabilities of a nonreacting species (e.g.; $\mathrm{N}_{2}$ or He) through aqueous solutions of DEA were estimated from pure component permeation studies, in the absence of $\mathrm{CO}_{2}$ gas, under conventional polymeric membrane mode in permeator \#3. 
Table 4-1

PERMEABILITIES OF PURE GASES THROUGH WATER AT DIFEERENT TEMPERATURES

\begin{tabular}{|c|c|c|c|c|c|}
\hline \multirow[t]{2}{*}{$\begin{array}{c}\text { Temp } \\
{ }^{\circ} \mathrm{C}\end{array}$} & \multirow[t]{2}{*}{ Gas } & \multirow[t]{2}{*}{$\begin{array}{l}D_{i w} X 10^{5} \\
\mathrm{~cm}^{2} / \mathrm{sec}\end{array}$} & $\begin{array}{r}\mathrm{H}_{\mathrm{iw}} \times 10^{4} \\
\operatorname{scc}(\mathrm{g}) \\
\end{array}$ & $\begin{array}{l}Q_{i} \times 10^{9} \\
\mathrm{scc}-\mathrm{cm} \\
\end{array}$ & \multirow{2}{*}{$\begin{array}{l}Q_{i} \times 10^{14} \\
\frac{m o l e-m}{m^{2}-s-P a}\end{array}$} \\
\hline & & & $\mathrm{cc}(\mathrm{w})-\mathrm{cm} \mathrm{Hg}$ & $\mathrm{cm}^{2}-\mathrm{s}-\mathrm{cm} \mathrm{Hg}$ & \\
\hline \multirow[t]{4}{*}{15} & $\mathrm{CO}_{2}$ & $1.4^{1}$ & $133.2^{2}$ & 186.5 & 62.40 \\
\hline & $\mathrm{N}_{2}$ & $2.2^{3}$ & $2.22^{2}$ & 4.88 & 1.63 \\
\hline & $\mathrm{He}$ & $6.2^{3}$ & $1.30^{2}$ & 8.06 & 2.69 \\
\hline & $\mathrm{CH}_{4}$ & $2.15^{3}$ & $4.87^{2}$ & 10.47 & 3.50 \\
\hline \multirow[t]{4}{*}{25} & $\mathrm{CO}_{2}$ & $1.92^{1}$ & $109.3^{2}$ & 210.0 & 70.27 \\
\hline & $\mathrm{N}_{2}$ & $3.0^{3}$ & $1.86^{2}$ & 5.58 & 1.86 \\
\hline & $\mathrm{He}$ & $7.4^{3}$ & $1.316^{2}$ & 9.74 & 3.26 \\
\hline & $\mathrm{CH}_{4}$ & $2.7^{3}$ & $3.97^{2}$ & 10.72 & 3.59 \\
\hline \multirow[t]{4}{*}{35} & $\mathrm{CO}_{2}$ & $2.4^{1}$ & $78.2^{2}$ & 187.7 & 62.81 \\
\hline & $\mathrm{N}_{2}$ & $3.9^{3}$ & $1.67^{2}$ & 6.51 & 2.18 \\
\hline & $\mathrm{He}$ & $8.4^{3}$ & $1.34^{2}$ & 11.25 & 3.76 \\
\hline & $\mathrm{CH}_{4}$ & $3.6^{3}$ & $3.38^{2}$ & 12.17 & 4.07 \\
\hline
\end{tabular}

Feferences : $\quad 1$. Thomas and Adams (1965)

2. Perry and Chilton (1973)

3. Wise and Houghton (1966) 
Table 4-2

PERMEABILITY OF $\mathrm{CH}_{4}$ THROUGH FULLY EXCHANGED CELGARD 2400 ILM $^{4}$ CONTAINING WATER AT $25^{\circ} \mathrm{C}$

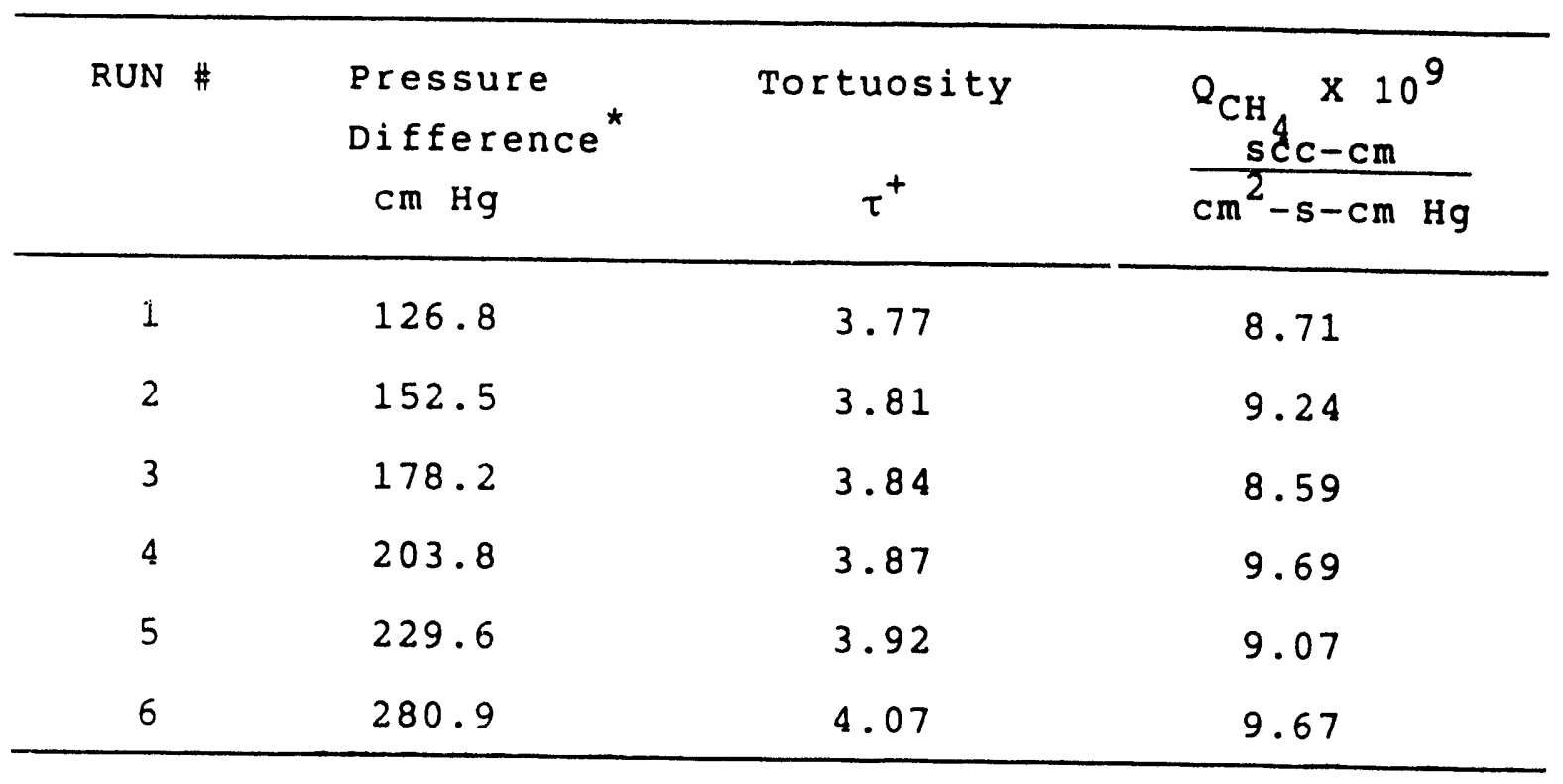
*Pressure Difference = Feed Pressure (pure $\mathrm{CH}_{4}$ )-Partial
+ Pressure of $\mathrm{CH}_{4}$ in Permeate

tortuosity values were take: from Bhave and sirkar (1986) 
The shell liquid was pressurized by a helium gas cylinder in all experiments. The differential equations (2-91) to (2-94) were solved simultaneously for different values of gas permeability. The correct species permeability should describe the observed pure component permeation behavior. The experimental data and the results from numerical analysis for $\mathrm{N}_{2}$ and He are presented in Tables 4-3 and 4-4, respectively. In all the calculations described above, the effective membrane thickness in permeator \#8 was considered to be 0.00485 inch. This particular value was determined from pure $\mathrm{CO}_{2}$ permeation studies through water as liquid membrane described later.

Permeability of a nonreacting species can also be estimated from the product of diffusivity and solubility through the solution. Sada et al. (1978) have studied the diffusivity and solubility of gases in aqueous solutions of amines. The diffusivity values of different gas species were estimated in two ways. In the first method, the variations of diffusivity of $\mathrm{N}_{2} \mathrm{O}$ in aqueous solution of DEA measured by Sada et al. (1978) were used. Diffusivity value was correlated with the concentration of DEA solution by the following equation :

$$
\left(D_{i A m} / D_{i w}\right)=\left(D_{A m} / D_{W}\right)_{N_{2} O}=1.0-0.11352 C_{T}
$$

where $C_{T}$ is the concentration of DEA in mole/l.

In the second method, diffusivity value was estimated by Stokes-Einstein equation using the viscosity values measured by Sada et al. (1978):

$$
\left(D_{i A m} / D_{i w}\right)=\left(\mu_{A m} / \mu_{W}\right)^{-1}
$$

Similarty, solubility value of $\mathrm{N}_{2} \mathrm{O}$ measured by Sada et al. (1977) was correlated by the following equation (Blanc and Demarais, 1984)

$$
\begin{aligned}
\text { In }\left(\mathrm{H}_{\mathrm{iAm}} / \mathrm{H}_{\mathrm{iw}}\right)=1.0406 \times 10^{-4} & +6.8433 \times 10^{-3} \mathrm{C}_{\mathrm{T}} \\
+ & 1.33633 \times 10^{-2} \mathrm{C}_{\mathrm{T}}^{2}-1.1549 \times 10^{-3} \mathrm{C}_{\mathrm{T}}^{3}
\end{aligned}
$$

Table 4-5 shows the estimated values and averaged experimental values (obtained flum Tables $4-3$ a $4-4$ ). Permeability of $\mathrm{CH}_{4}$ was not determined using pure component permeation method beacuse of safety reasons. Higher experimental 


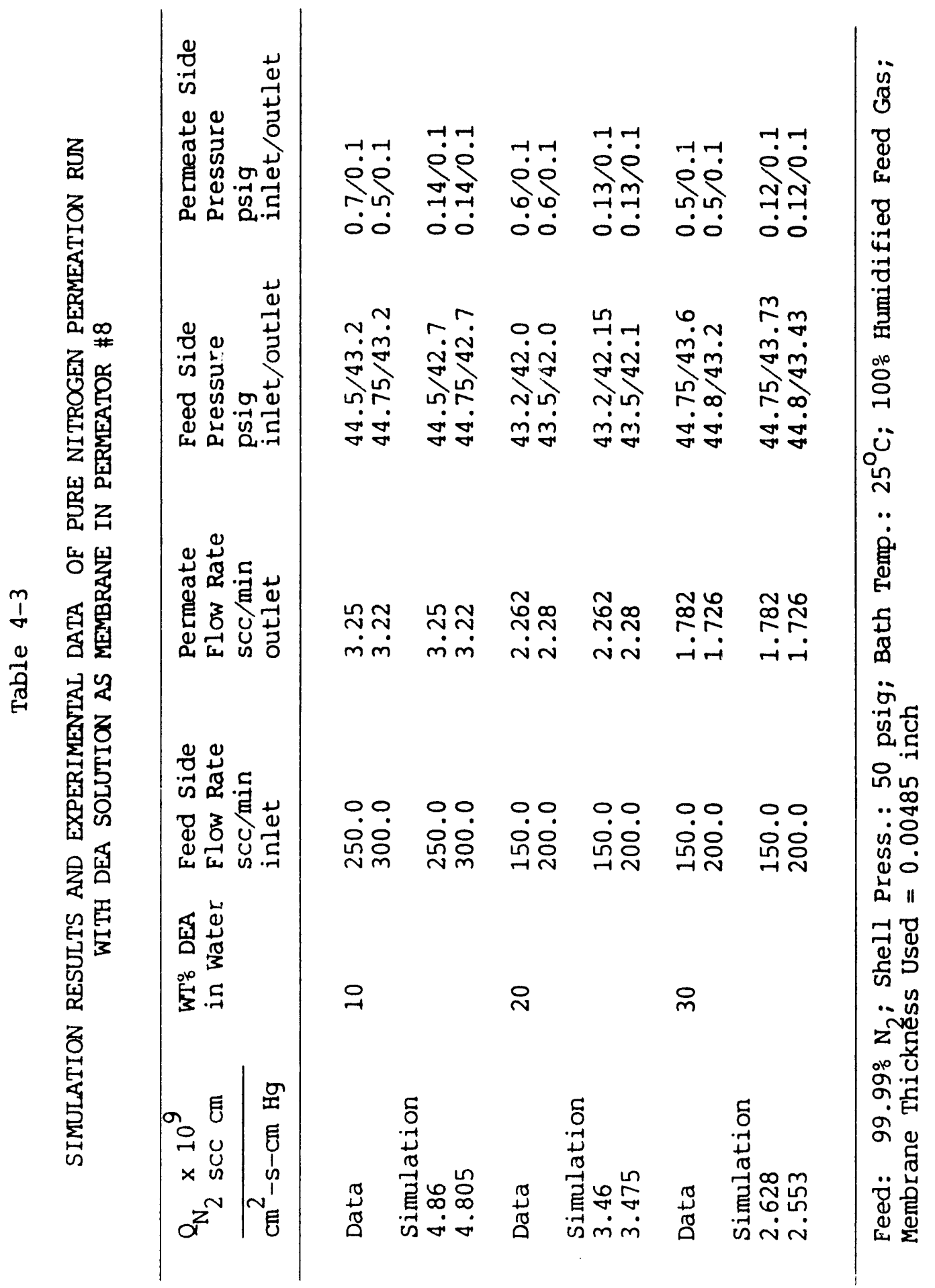




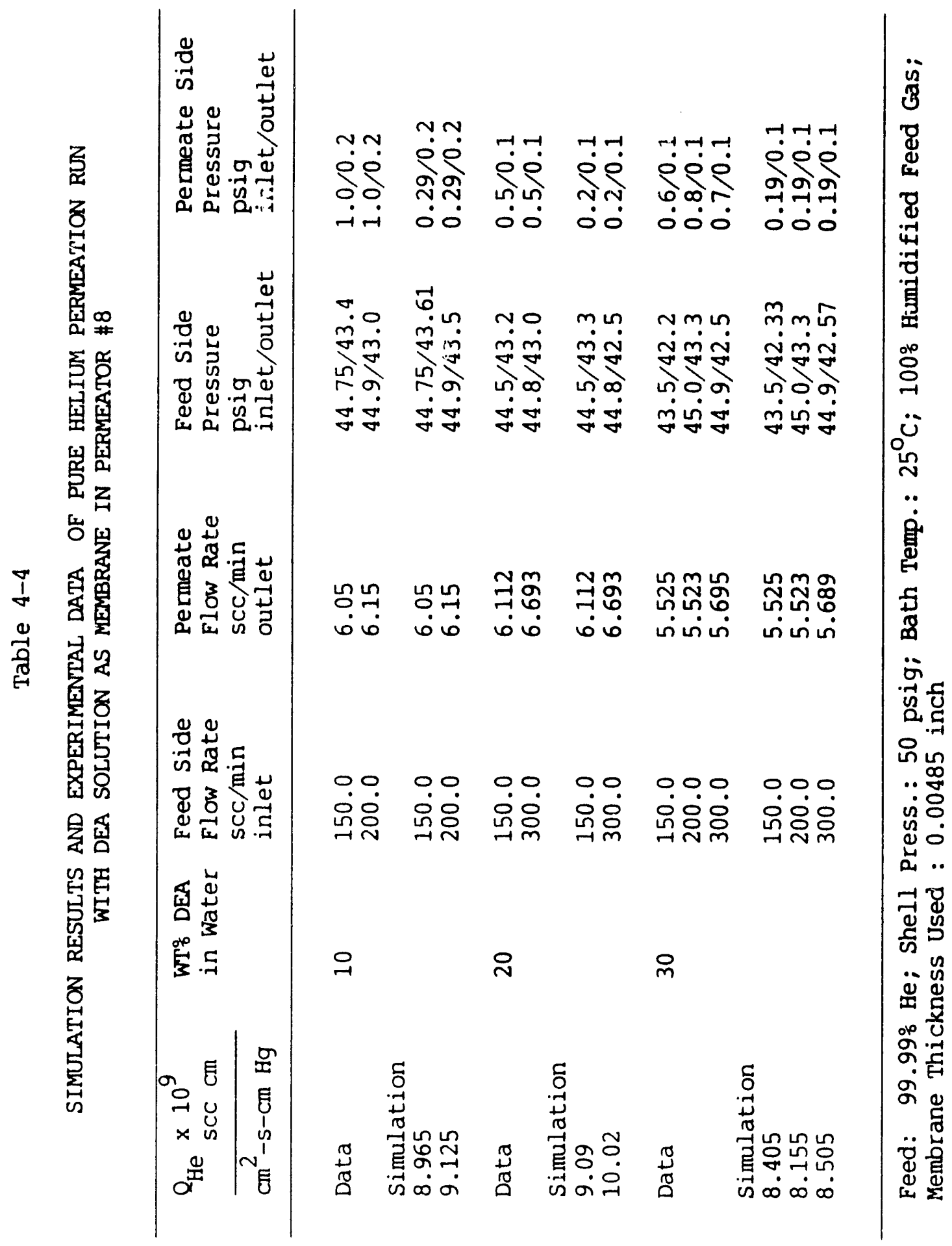




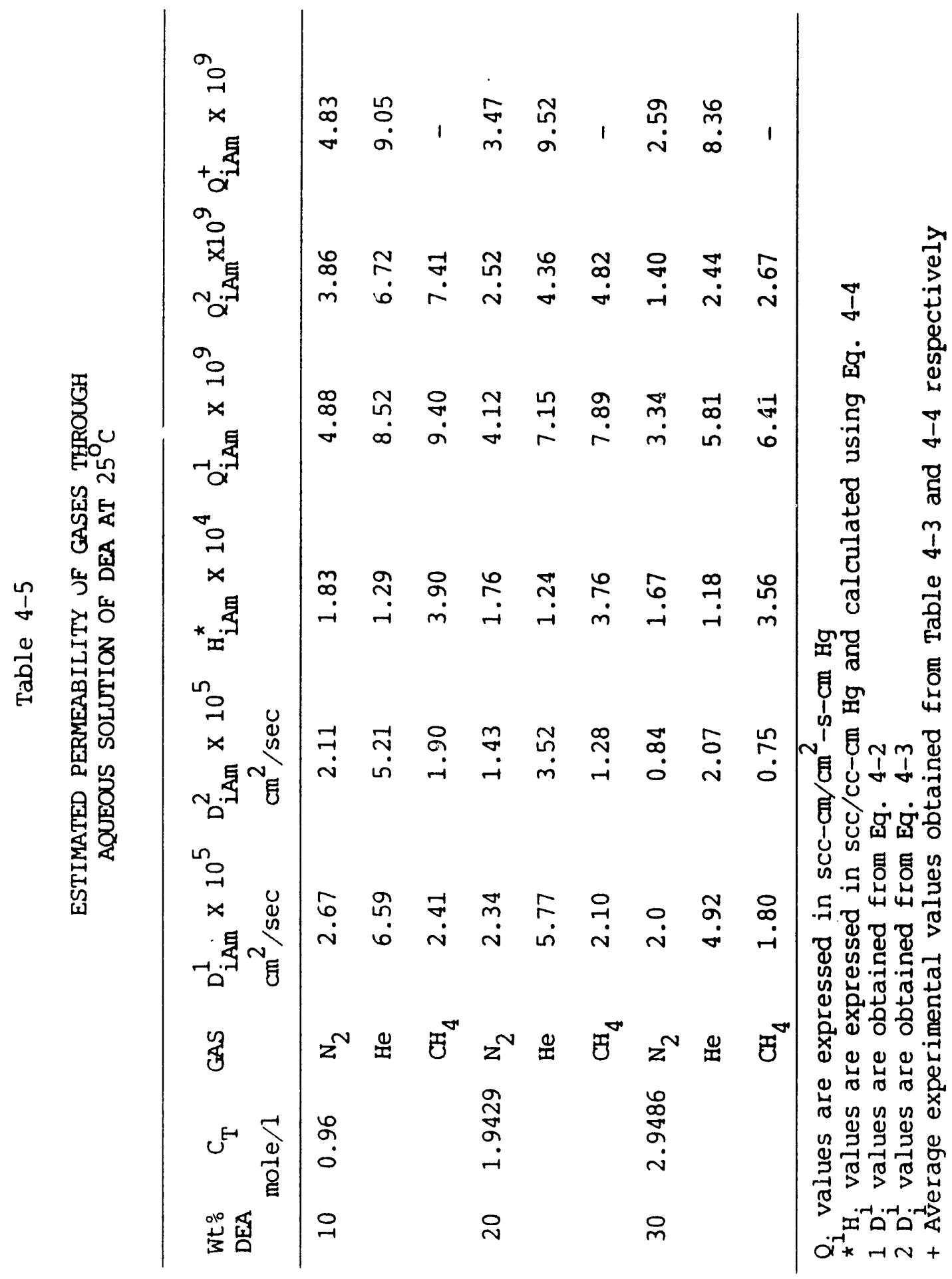


value compared to astimated value for He could be explasued by the fact that the size and the molecular weight of He are muct smiler than those of $\mathrm{N}_{2} 0$; therefore, $\mathrm{N}_{2} \mathrm{O}$ is not the right choice to predi: the permeability of he. In. for all the numerical s mulations, the experimental permeatil. y values for to were used.

The measurement of permeability of $\mathrm{CO}_{2}$ was carried out using the immobilized liquid membrane (ILM) technique. An indeperdent measurement of tortuosity factor of Celgard 2400 support $\mathrm{film}$ was first made at different pressures using pure $\mathrm{N}_{2}$ gas permeation through such a film having pure water $1 \|$ its pores. An average tortuosity value of 5 was obtained for the pressure range studied.

Table 4-6 indicates the permeation and separation behavior of $\mathrm{CO}_{2}-\mathrm{N}_{2}$ mixtures through fully exchanged Celgard ILM containing an aqueous 20 wt\% DEA solution (it is reported later that $20 \mathrm{wt} \%$ DEA solution gave the best separation behavior in HFCLM permeator in the partial pressure range of $\mathrm{CO}_{2}$ being studied). Two different gas mixtures containing $5 \% \mathrm{CO}_{2}-95 \% \mathrm{~N}_{2}$ and $25 \% \mathrm{CO}_{2}-75 \%$ $\mathrm{N}_{2}$ were used as feed. The total pressure of the feed mixture was kept between $231.14 \mathrm{~cm} \mathrm{Hg}$ and $515.57 \mathrm{~cm} \mathrm{Hg}$. Note that, the partial pressure range of $\mathrm{CO}_{2}$ covers the entire range studied for separation experiments in the HFCLM permeator. From the measured permeation rate of $\mathrm{N}_{2}$ and $\mathrm{CO}_{2}$, the permeability values of $\mathrm{N}_{2}$ and $\mathrm{CO}_{2}$ through $20 \mathrm{wt} \%$ DEA solution were calculated using the following relation:

$$
D_{i}=\left|R_{i} \times t_{m} \times \tau_{m}\right| /\left|\Delta P_{i} \times \varepsilon_{m} \times A_{m}\right|
$$

The nitrogen permeability value $\left(1.75 \times 10^{-9} \mathrm{scc}-\mathrm{cm} / \mathrm{cm}^{2}-\mathrm{s}-\mathrm{cm} \mathrm{Hg}\right)$ obtained in this set of measurements is about half of that $\left(3.4675 \times 10^{-9} \mathrm{scc}-\mathrm{cm} / \mathrm{cm}^{2}-\mathrm{s}-\mathrm{cm}\right.$ $\mathrm{Hg}$ ) obtained earlier in our pure component permeation runs in the permeator \#8 (Table 4-3). Note that pure component permeation runs were carried out in the absence of $\mathrm{CO}_{2}$ gas. When $\mathrm{CO}_{2}$ dissolves in the aqueous solution of $\mathrm{DEA}$, the following overall reaction occurs:

$$
\mathrm{CO}_{2}+2 \mathrm{R}_{2} \mathrm{NH}=\mathrm{R}_{2} \mathrm{NCOO}^{-}+\mathrm{R}_{2} \mathrm{NH}_{2}^{+}
$$

It is possible that when $\mathrm{CO}_{2}$ is present along with $\mathrm{N}_{2}$, the $\mathrm{N}_{2}$ permeability 


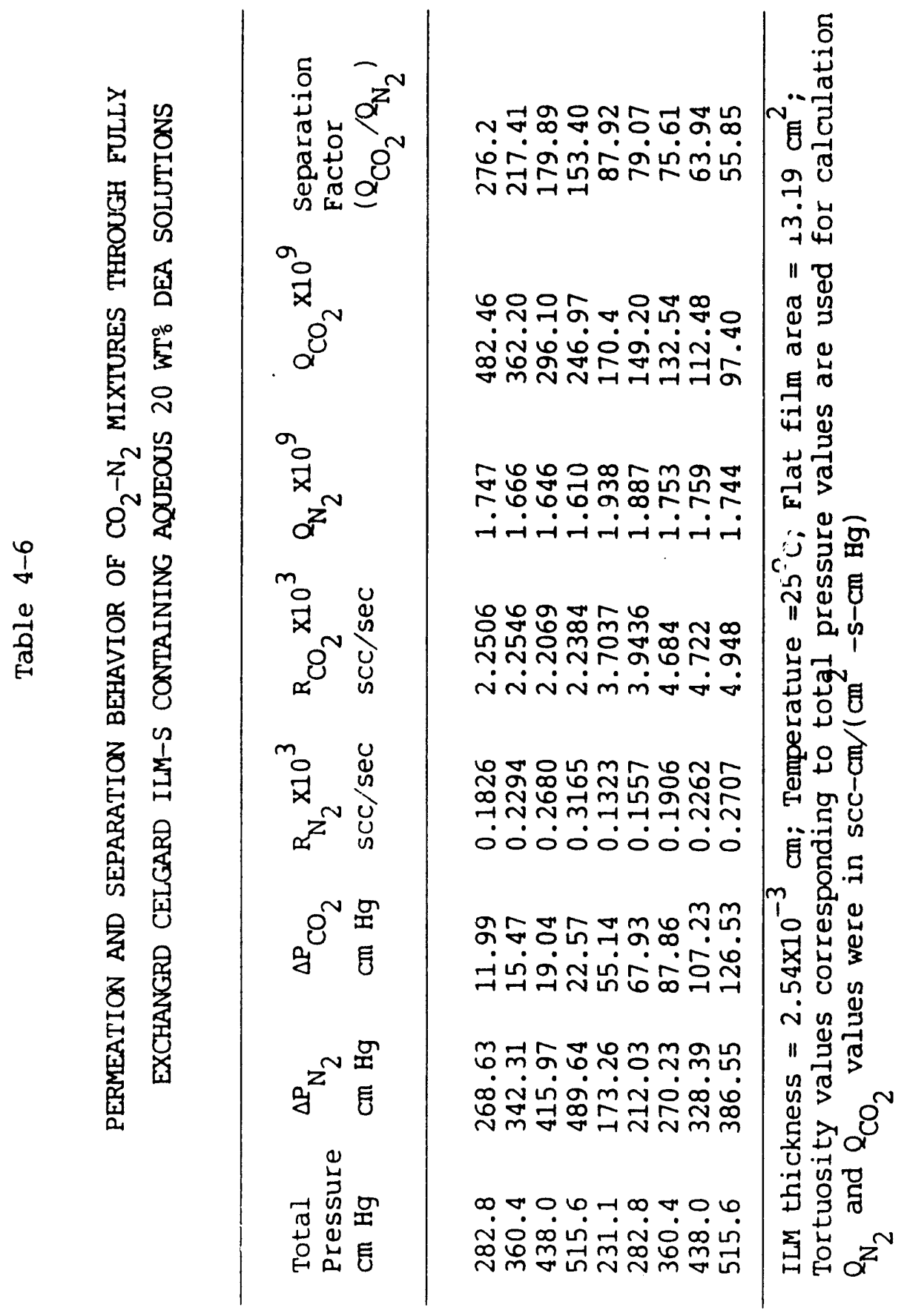


value reduces further due to the presence of other ions e.g., $\mathrm{R}_{2} \mathrm{NCOO}^{-}$and $\mathrm{R}_{2} \mathrm{NH}_{2}^{+}$ in the liquid membrane. Moreover, relative amounts of association among this polar intermediate further lowers the permeability (Donaldson and Nguyen, 1980). However, the estimated value of $2.52 \times 10^{-9} \mathrm{scc}-\mathrm{cm} / \mathrm{cm}^{2}-\mathrm{s}-\mathrm{cm} \mathrm{Hg}$ from Table 4-5 for $\mathrm{N}_{2}$ lies in between the above two values. Thus it is expected that the values listed in the eighth column of this table for $\mathrm{N}_{2}$ and $\mathrm{CH}_{4}$ would be a better choice to analyze the actual separation data in HFCLM permeator.

The observed separation factors of $\mathrm{CO}_{2}$ and $\mathrm{N}_{2}$ through 20 wt\% DEA solution at different partial pressures of $\mathrm{CO}_{2}$ have been compared with the numerical simulation results based on the analysis discussed in Section 2 and presented in Table 4-7. Though the present model predicted the separation factors quite well as shown in Figure $4-1$, the permeability values of $\mathrm{CO}_{2}$ were always overpredicted (see Tables 4-6 and 4-7) to a great extent. This could be due to uncertainties in the estimation of other parameters, such as, diffusivity of amine, rate constants and reaction orders with respect to DEA which have considerable effects on the prediction (Donaldson and Nguyen, 1980). Experimental diffusivity of amine (Thomas and Furzer, 1962), measured in the absence of reaction, was used. The equilibrium constants $\mathrm{K}_{\mathrm{Am}}$ and $\mathrm{K}_{\mathrm{p}}$ at $20^{\circ} \mathrm{C}$ were obtained from Danckwerts and Sharma (1966), who reported that the values of the equilibrium constants are influenced substantially by the ionic strength of the solution; it is not generally possible to make accurate allowance for this. Therefore, those values were used for the present analysis. In addition, it should be remembered that other investigators have studied only the forward reaction in the absence of significant carbamate product which will have a noticable effect on all these parameters.

\section{ESTIMATION OF EPFECTIVE MEMBRANE THICKNESS IN SMALL PERMEATORS}

To determine the effective membrane thickness (EMT) experimentally, pure $\mathrm{CO}_{2}$ permeation studies under conventional mode of operation with water as liquid membrane have been carried out. The governing equations for pure gas permeation (described in Section 2) were solved for different values of effective membrane thickness. The correct value should match the permeation rate of $\mathrm{CO}_{2}$ obtained experimentally. The pure component permeation data for permeators \#3, \#6 and \#8 are compared with the numerical results in Tables 4-8, 4-9 and 4-10, and the 


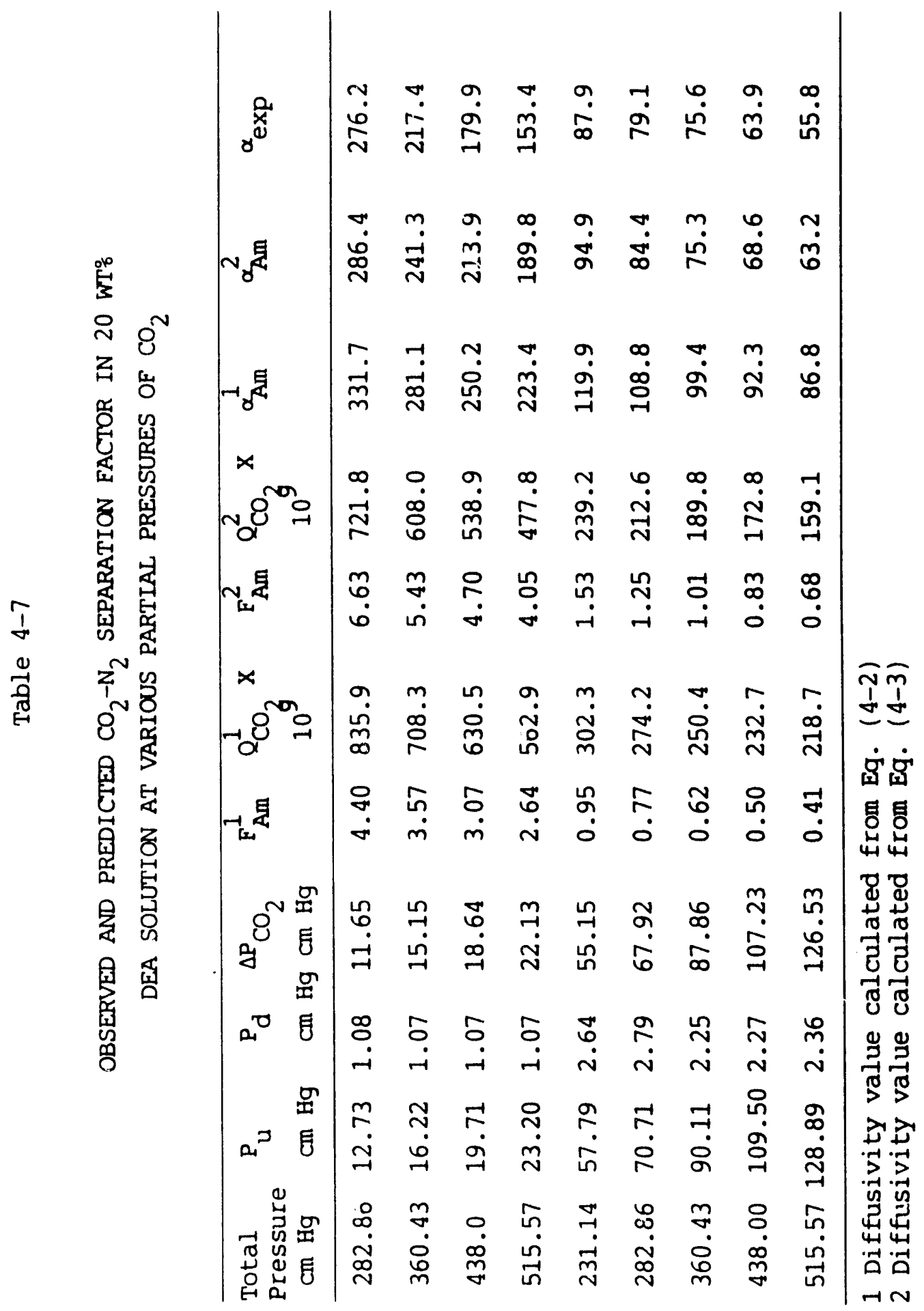




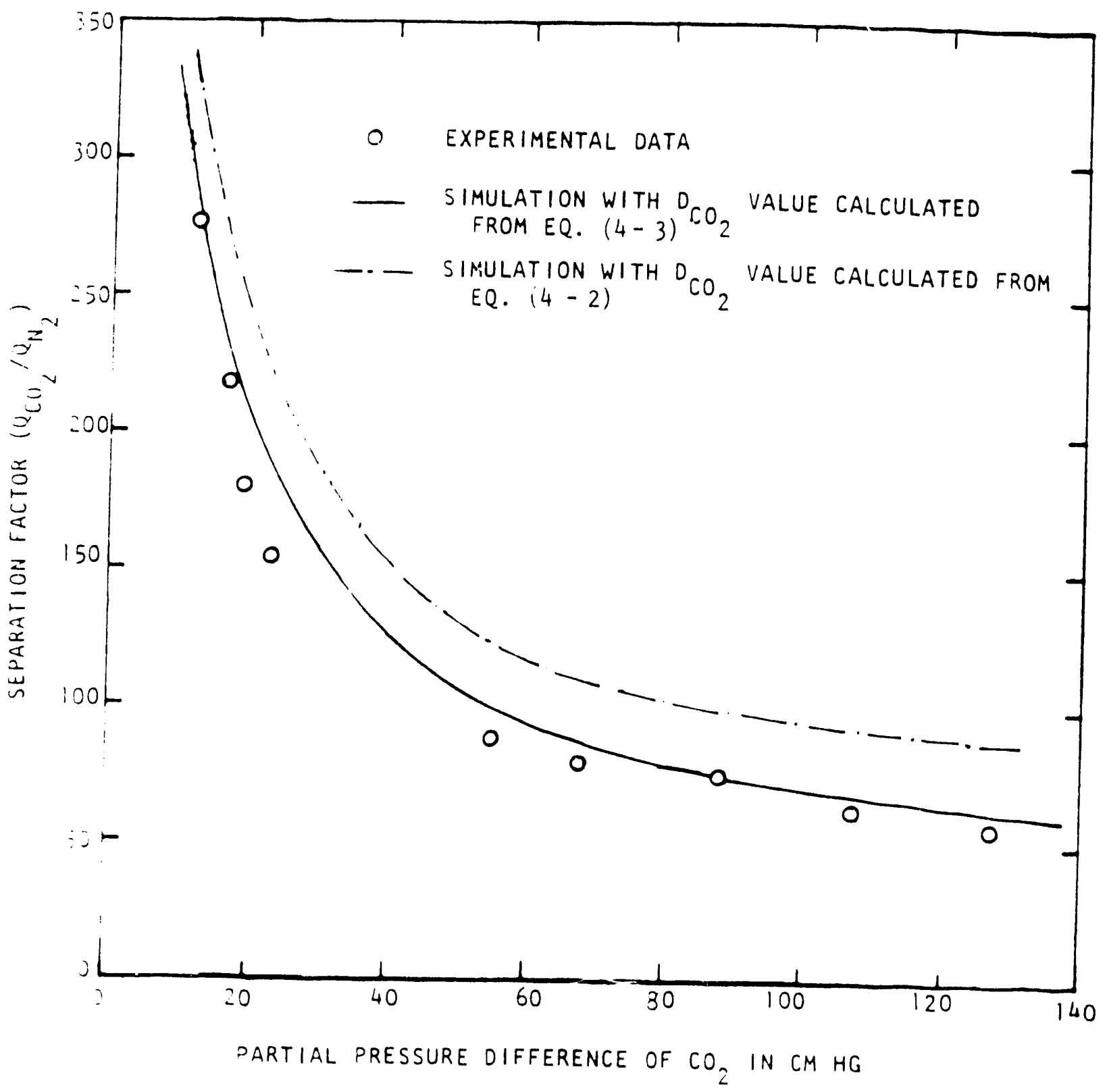

FIGURE $4-1$

OBSERVED AND PREDICTED SEPARATION FACTOP

OF $\mathrm{CO}_{2}-\mathrm{N}_{2}$ THROUGH AQUEOUS 20 WT 


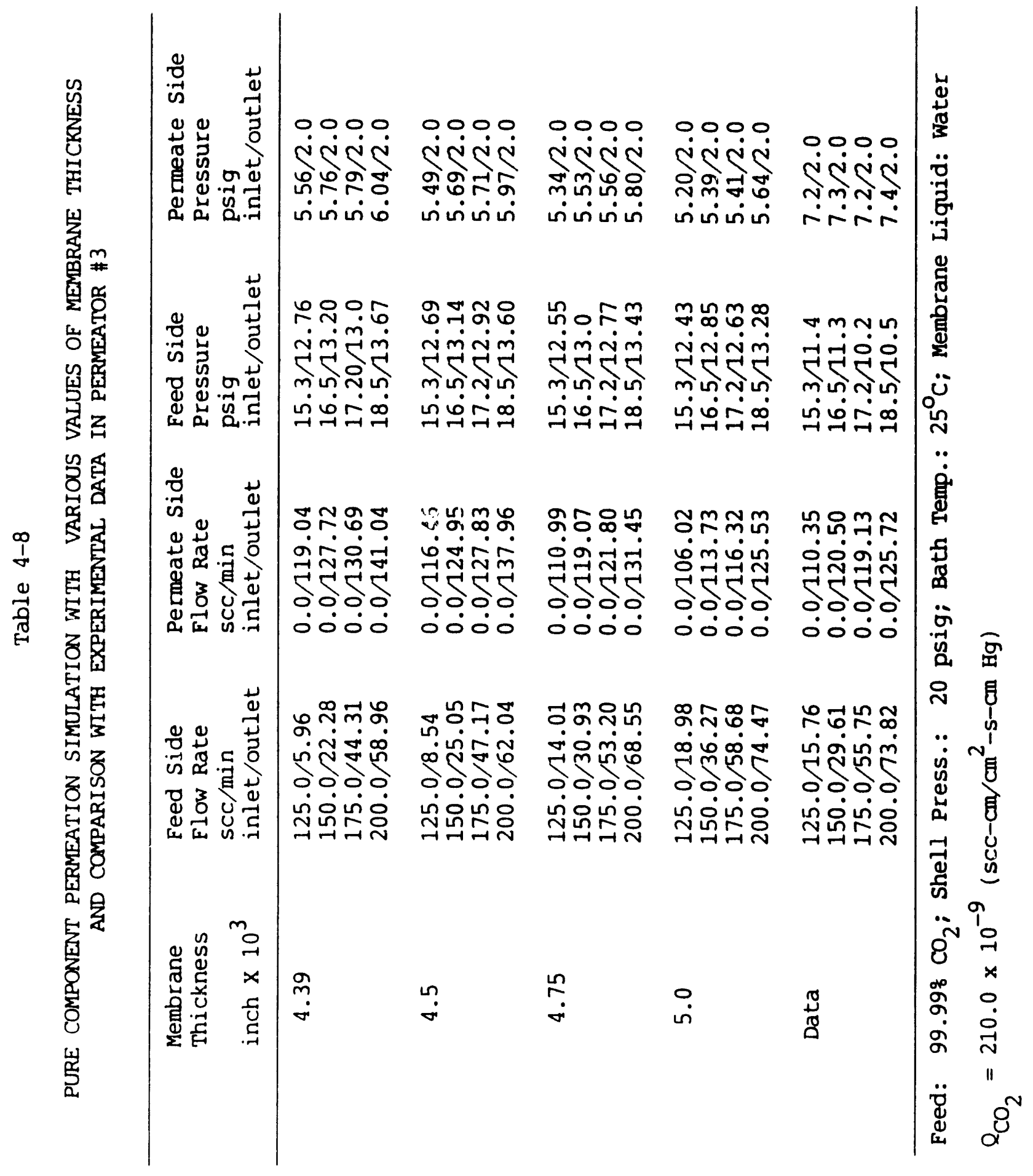




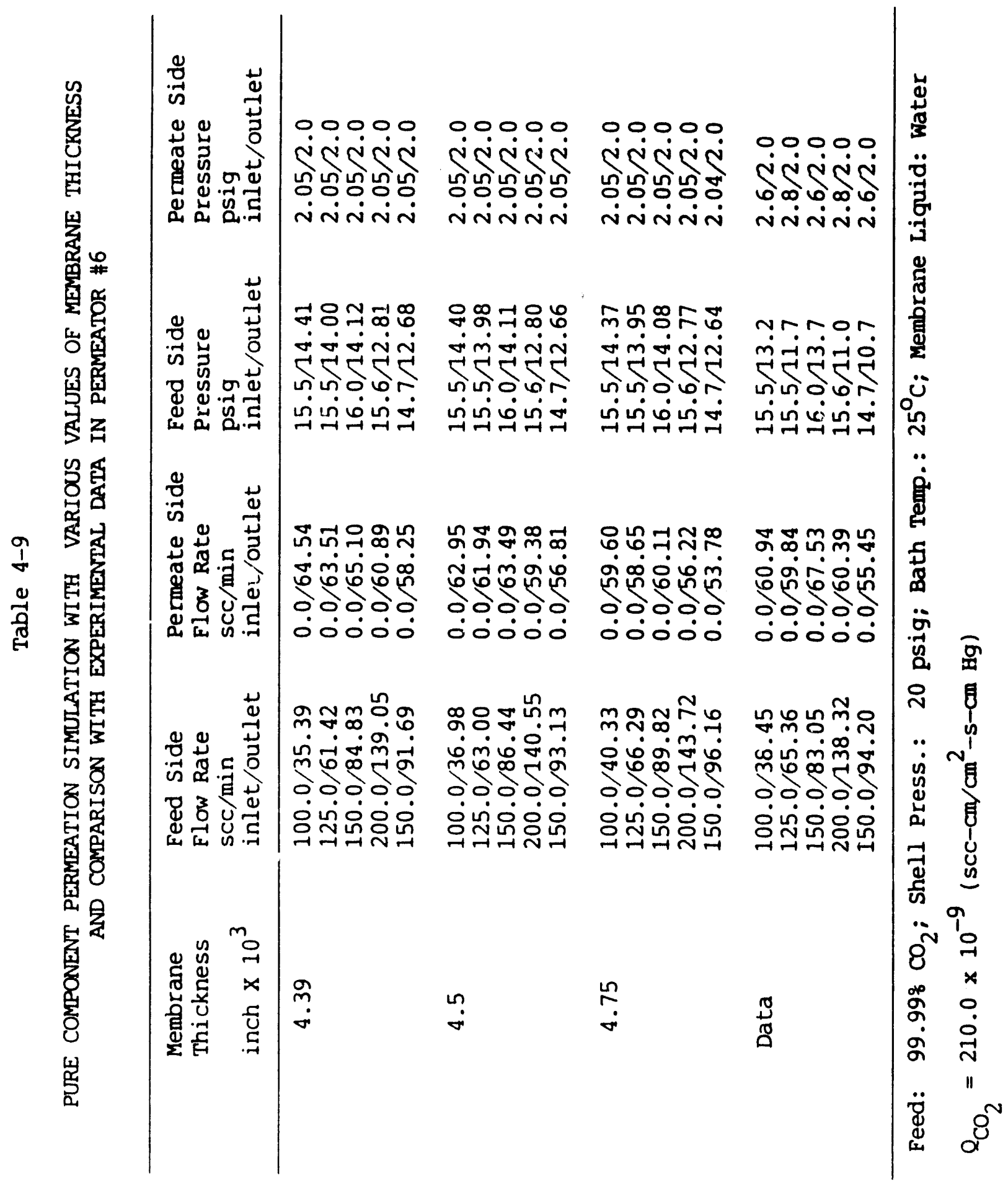




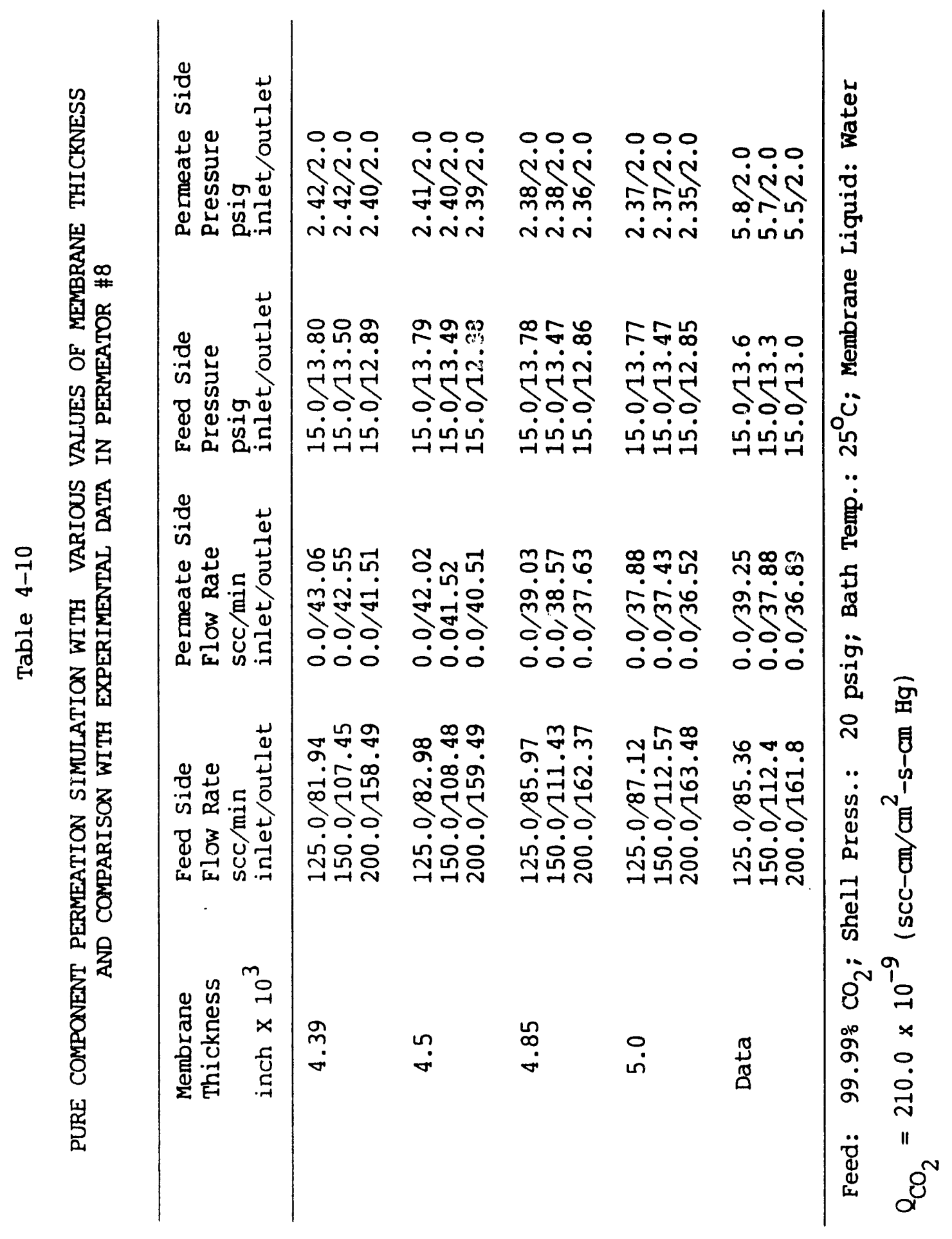


effective membrane thicknesses are estimated to be 0.00475 inch, 0.0045 inch and 0.00485 inch, respectively.

The EMT can also be determined from actual separation run through water in the sweep gas mode by solving the three-component permeation model for several assumed values of $d$. The correct value should give a good agreement between calculated values and observed data. Table 4-11 shows the separation data and simulation results for Permeator \#5. An EMT value of 0.00439 inch matched the data very well. As will be shown later, except for permeator \#6, the EMT values for different permeators, thus determined, were in close agreement with the values determined from pure component permeation runs.

Table 4-12 shows that, for permeator \#6, a value of $d=0.01$ inch gives a better agreement between observed data and calculated values. The discrepancy from the value obtained by pure component permeation run for permeator \#6 is most likely due to the leakage from the defective fiber as well as condensation of water in the pores of the microporous fiber wall. This increased the resistance for mass transfer which is reflected in the higher membrane thickness. Moreover the separation runs were carried out at higher feed gas inlet pressure which required higher shell liquid pressure (note that, in a HFCLM permeator, the shell pressure is always maintained at a higher value than the gas streams pressures). Since the pressure drop in the sweep side was not that much, the larger diameter fibers were exposed to the high pressure differentials; that caused deformation of the larger diameter fibers. This deformation has not been incorporated in the three-component permeation model. When pressurized externally, single hollow fiber undergoes two types of deformation: radial and axial (Stern et al., 1977; Thorman and Hwang, 1978). As a result, the fiber cross section decreases and the length of the fiber increases. Both of these should cause an increase in the pressure drop in the larger bore fibers (discussed in separation studies section later). Apart from these, as the diameter decreases, the permeation rate due to reduction in cross sectional area decreases, which in turn increases the effective membrane thickness. The effect was not prominent in the case of smaller diameter fibers which are considered to be 'thick cylinders' (wall thickness: 25 micron, ID : 100 micron). Thus 0.01 inch membrane thickness was used in all numerical simulations of the performance of permeator \#6. The effective membrane 


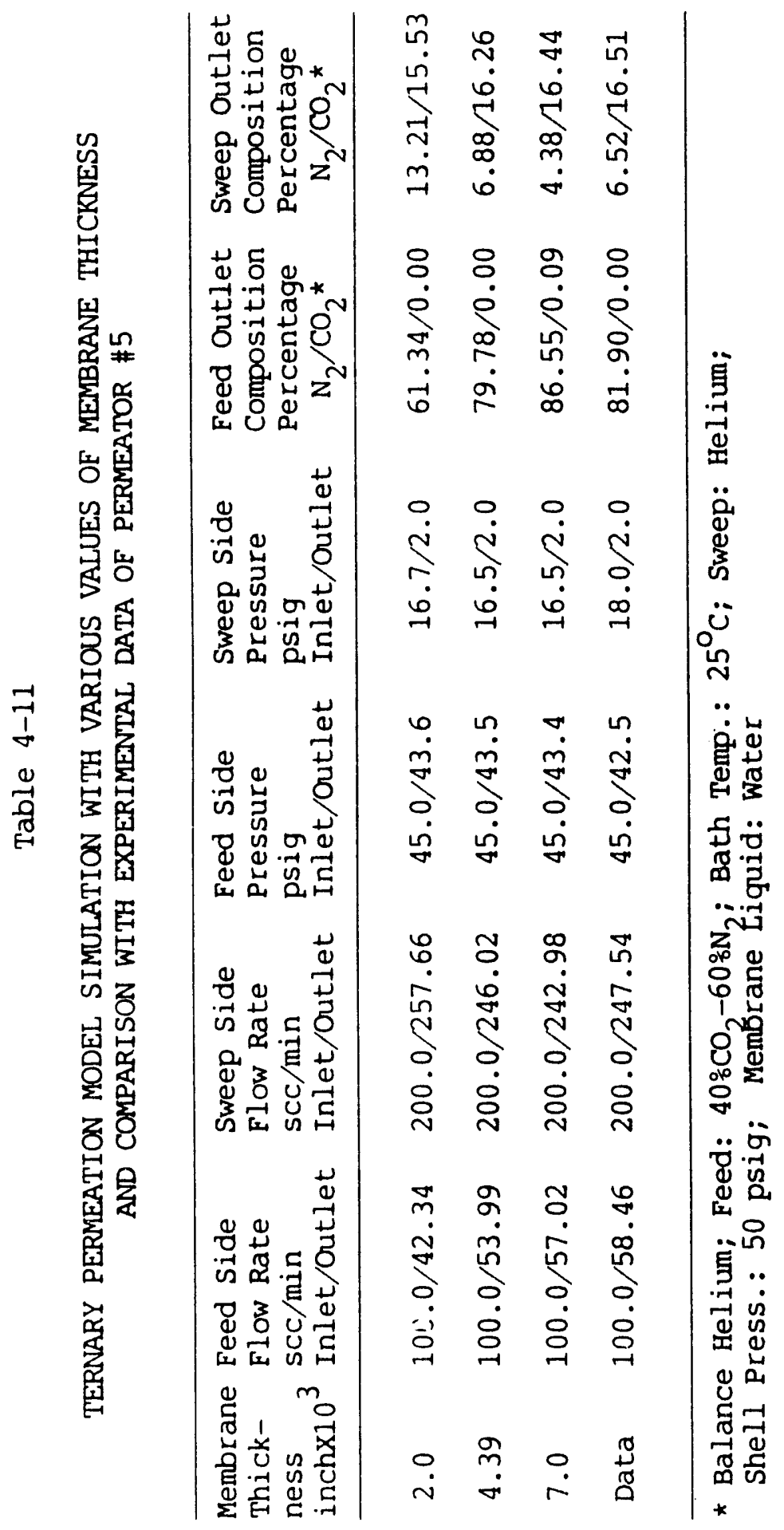




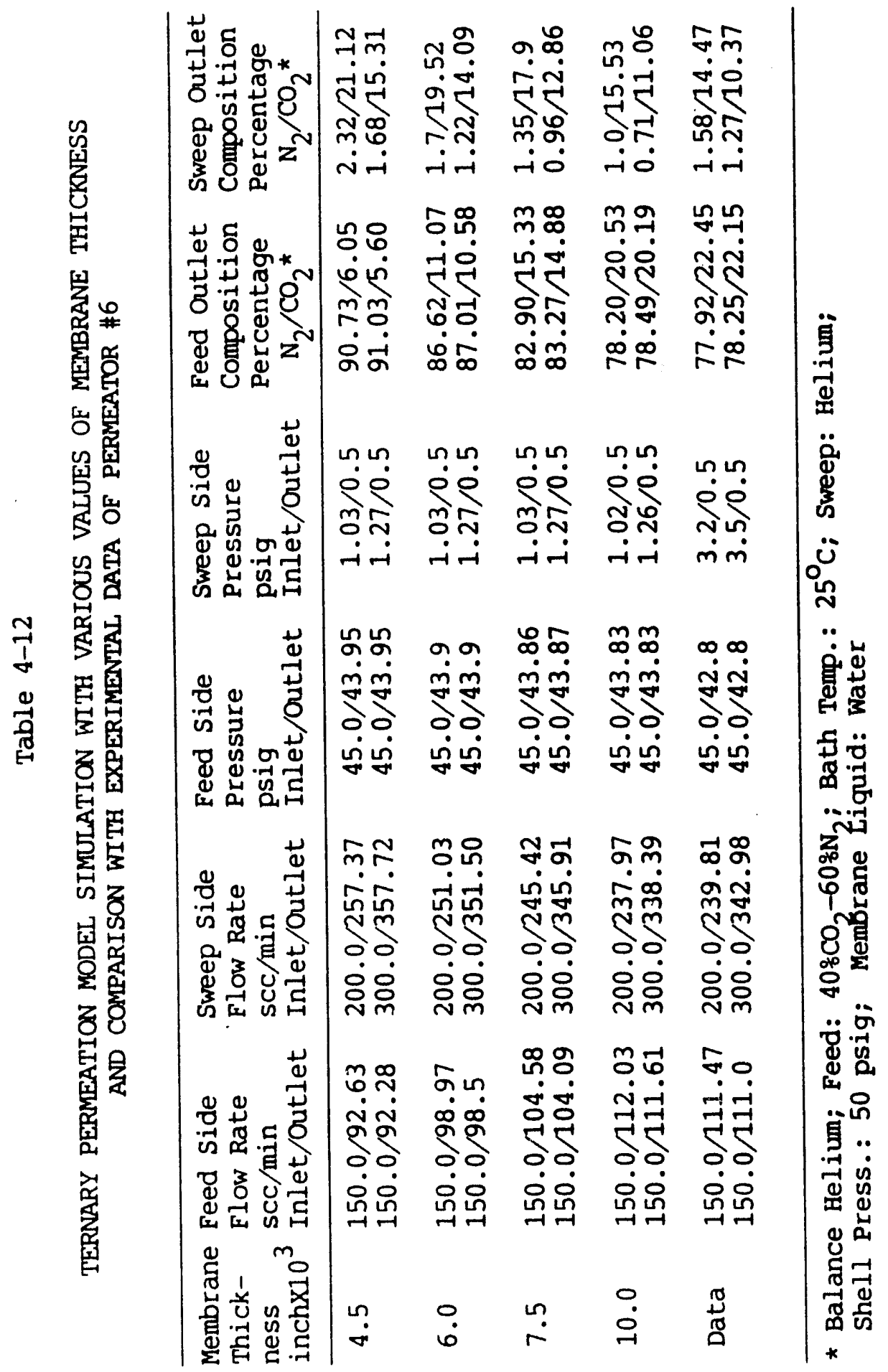


thickness values of different permeators are reported in Table 4-13.

The EMT may be determined theoretically by solving the two-dimensional diffusion equation for a chemically non-reactive gas membrane system (Majumdar et al., 1989; NYSERDA Report 87-10). The analysis was based on the assumption that hollow fibers are arranged in square pitch. Thus for permeators \#3, \#4, \#5 and $\# 8$, having the same packing density and fiber OD, a single EMT value of 0.00439 inch was calculated (Majumdar et al., 1989; NYSERDA Report 87-10). This theoretical prediction is in close agreement with the value obtained from pure component premeation run and from separation run.

Utilizing the generalized two-dimensional model, the effect of hollow fiber outside diameter and packing density on average effective liquid membrane thickness were calculated. The computed results, plotted in Figure 4-2, suggest that, the membrane thickness decreases almost linearly with decrease in fiber $O D$ for a particular value of packing density. The same result was obtained by increasing the packing density while the fiber $O D$ was kept constant. Therefore, it is possible to reduce the EMT value for HFCLM permeators provided hollow fibers of smaller OD are available.

PURIFICATION STUDIES OF MODEL BIOGAS MIXTURES IN SUEEP GAS MODE THROUGG DIFPERENT LIQUID MEMBRANBS IN SMALL-SCALE OPERATION

Model biogas purification studies were carried out with different liquid membranes such as pure water, aqueous $\mathrm{K}_{2} \mathrm{CO}_{3}$ solution and aqueous solution of DEA. For pure water as membrane, the gas streams were not humidified. However, the streams were humidified completely when aqueous solution of either $\mathrm{K}_{2} \mathrm{CO}_{3}$ or DEA was used as membrane. All experiments were carried out at $25^{\circ} \mathrm{C}$. Pure helium gas was normally used to pressurize the shell liquid storage tank.

The material balances were checked from the tables shown later and found to be within the error limit $( \pm 5 \%)$ of the electronic mass flowmeter readings. Thus the material balances were considered to be satisfactory. In all the figures, feed and sweep outlet mole fractions of $\mathrm{CO}_{2}$ and $\mathrm{N}_{2}$ or $\mathrm{CH}_{4}$ are designated by $\mathrm{CO}_{2} / \mathrm{F}, \mathrm{N}_{2} / \mathrm{F}$ or $\mathrm{CH}_{4} / \mathrm{F}$ and $\mathrm{CO}_{2} / \mathrm{S}, \mathrm{N}_{2} / \mathrm{S}$ or $\mathrm{CH}_{4} / \mathrm{S}$, respectively. 
Table 4-13

EFFECTIVE MEMBRANE THICKNESS *

FOR DIFFERENT SMALL PERMEATORS

\begin{tabular}{cc}
$\begin{array}{c}\text { PERMEATOR } \\
\text { NO. }\end{array}$ & $\begin{array}{c}\text { THICKNESS } \\
\text { MIL }\end{array}$ \\
\hline 3 & 4.39 \\
5 & 4.39 \\
6 & 10.00 \\
8 & 4.85 \\
\hline
\end{tabular}

* Determined from experimental separation data 

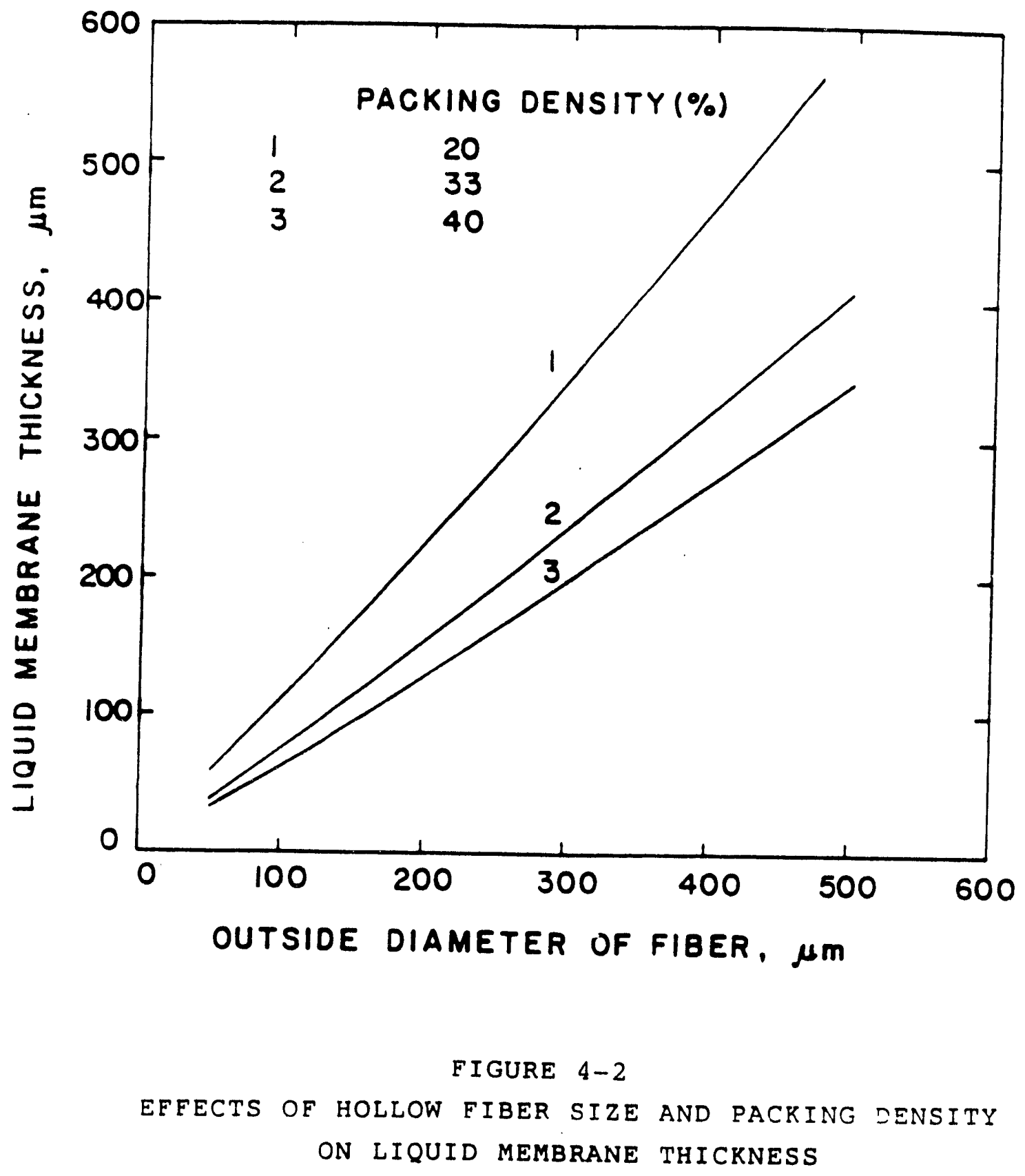
Water as Liquid Membrane and $40 \% \mathrm{CO}_{2}-60 \% \mathrm{~N}_{2}$ as Peed Gas

Steady state separation data for a model biogas feed mixture separation through pure water membrane in permeator \#5 was already reported in Table 4-11. The nitrogen recovery (defined as the percentage of the fractional volume of nitrogen retained in the purified feed gas) was $79.8 \%$. The data were used to determine the effective membrane thickness in the permeator. This value of membrane thickness was also obtained for permeator \#3 (Majumdar, 1986; NYSERDA Report 87-10). We had carried out extensive separation studies in this mode using permeator \#3 with water as liquid membrane. Thus it is expected that permeator \#5 would give similar behavior as long as water is being used as liquid membrane.

Separation studies of the model biogas mixture in this mode with permeator \#6 containing mixed fibers were carried out at two different pressures with pure water as membrane. To reduce the sweep stream pressure drop, the larger diameter fibers were used as sweep channel. The steady state data for a high feed inlet pressure ( 45 psig) are presented in Table 4-14. Both feed and sweep flow rate variations are shown. For feed flow variation studies, feed flow rates of 50 to $150 \mathrm{sccm}$ were maintained. Pressure drop in the sweep side at the highest sweep gas flow rate of $400 \mathrm{sccm}$ was only around 4 psi. It was possible to bring down the $\mathrm{CO}_{2}$ concentration level to around $10 \%$ at the lowest feed flow rate. Note that, the effective length of the permeator is only 2 feet, i.e., it has only $40 \%$ of the membrane area of permeator \#5. Due to the much lower sweep side pressure drop, helium permeation rate to the feed stream was quite low in this case when compared with that in permeator \#5 as well as the earlier studies in permeators containing smaller diameter fibers (Majumdar et al., 1988). Nitrogen recovery was more than $90 \%$ in all the cases.

The data and the simulation results for feed gas flow variation studies are shown in Figure 4-3; those for sweep flow variation studies are shown in Figures 4-4 and 4-5 for two different feed flow rates where a membrane thickness of 0.01 inch was used for simulation. The simulation explains the data trends quite well. As expected, the $\mathrm{CO}_{2}$ percentage in the purified gas increased with an increase in feed flow rate. 


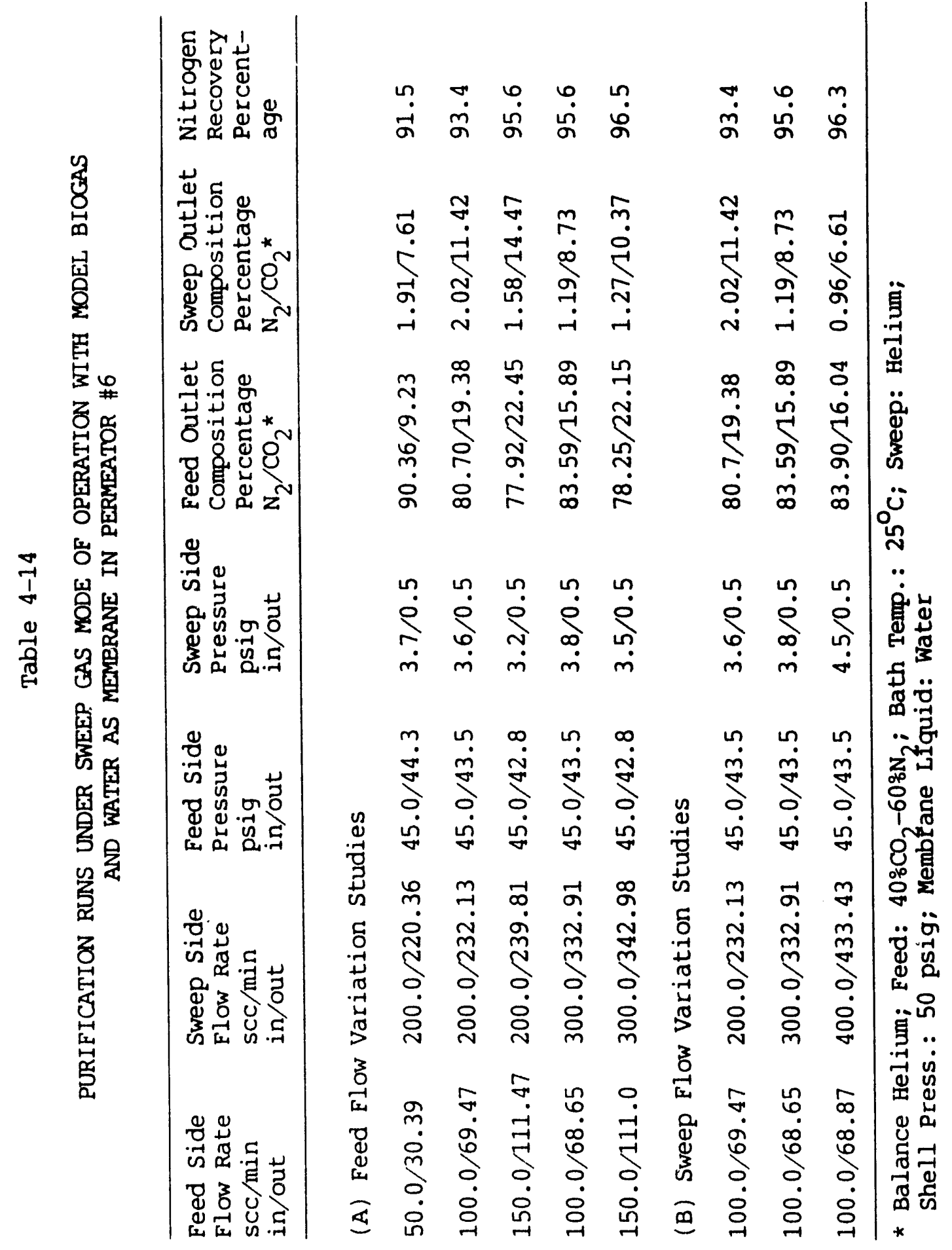



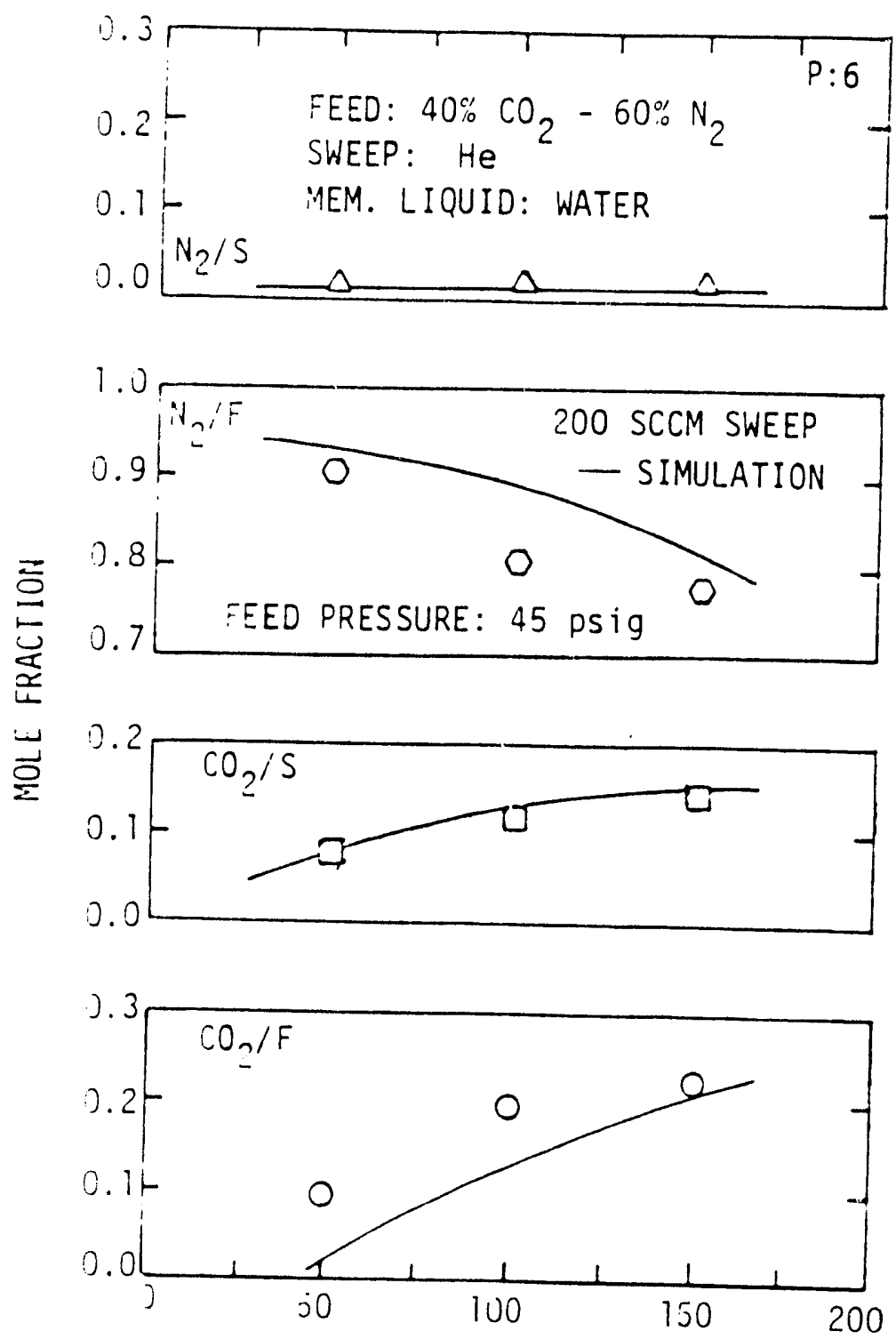

FEED FLOW RATE SCC/MIN

FIGURE 4-3

FEED FLOW VARIATION STUDIES:

SWEEP GAS MODE OF OPERATION IN PERMEATOR \#6 

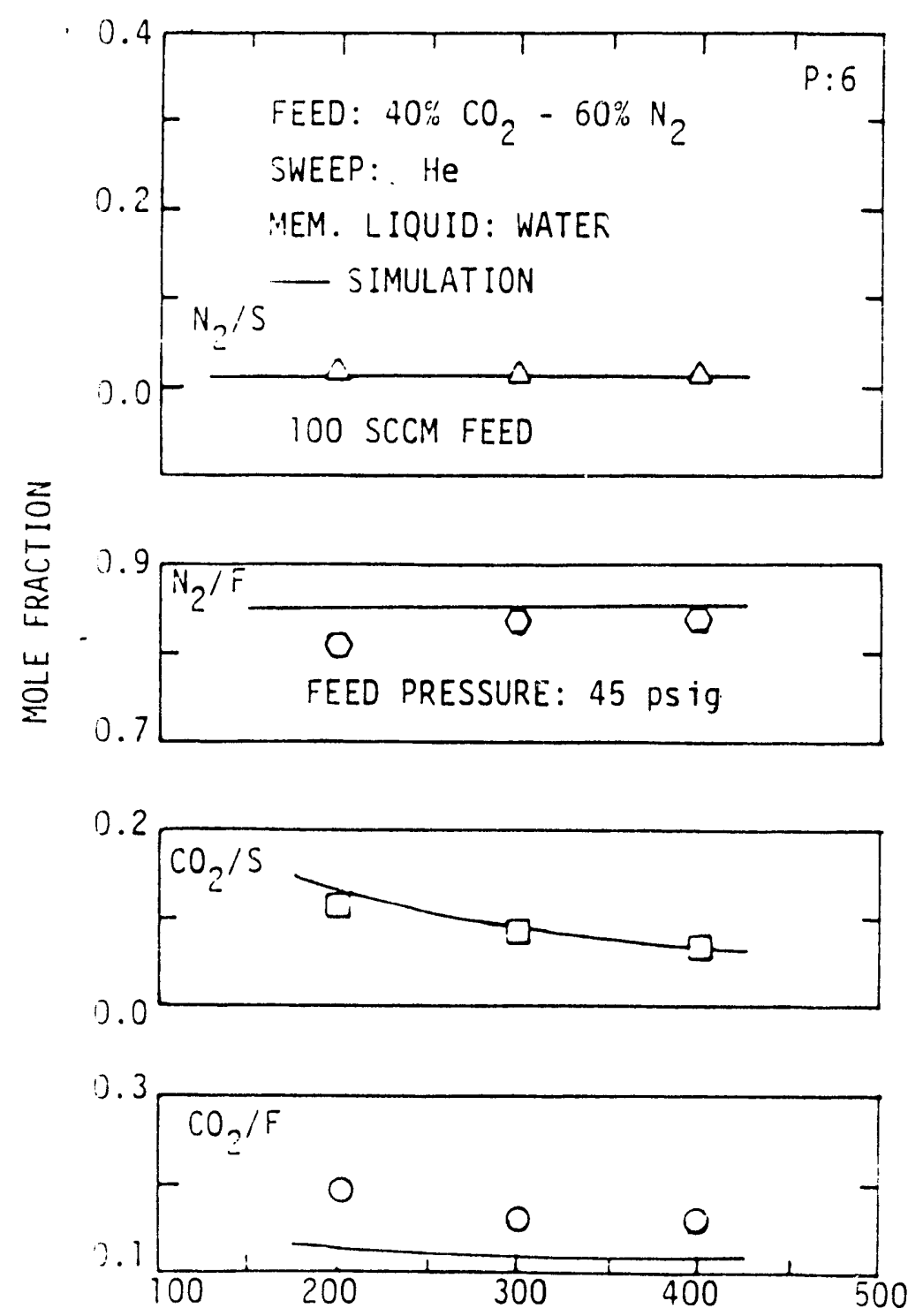

SWEEP FLOW RATE SCC/MIN

FIGURE $4-4$

SWEEP FLOW VARIATION STUDIES:

SWEEP GAS MODE OF OPERATION IN PERMEATOR \#6 

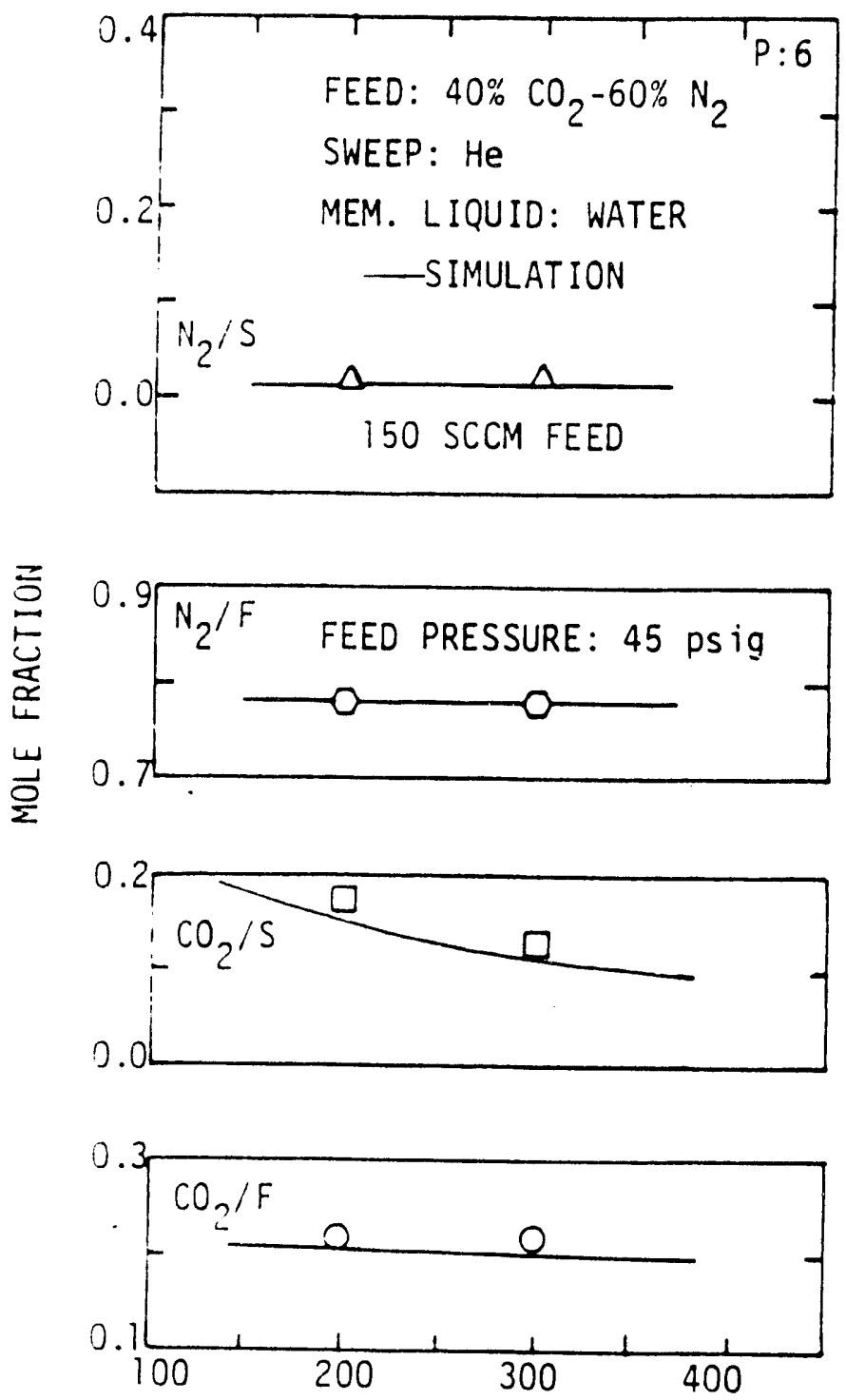

SWEEP FLOW RATE SCC/MIN

FIGURE 4-5

SWEEP FLOW VARIATION STUDIES:

SWEEP GAS MODE OF OPERATION IN PERMEATOR \# 6 
Since the pure component permeation run was carried out at low pressure $(\sim 15$ psig) the separation run was also carried out at low pressure to study the effect of shell pressure. Steady state results for feed flow variation studies at a low pressure ( 16 psig) are presented in Table 4-15. The data and the simulation results for feed gas flow variation studies are compared in Figure 4-6 where again a membrane thickness of 0.01 inch was used for simulation. These results suggest that leakage of liquid as well as fiber deformation were still there. Note that, compared to the long permeator, the permeate fibers in permeator \#6 were always exposed to higher membrane liquid pressure ditferential because of lower pressure drop. Comparing Tables 4-14 and 4-15 we see that the helium content in purified feed gas increased as the feed gas pressure was lowered. Higher sweep stream pressure drop in Table 4-14 suggests more leakage and deformation at higher shell liquid pressure.

The results of feed and sweep flow variation studies in permeator \#8 are shown in Table 4-16. The $\mathrm{CO}_{2}$ percentage in the purified gas stream varied from 6 $12 \%$, depending on the feed and sweep gas flow rates. The $\mathrm{N}_{2}$ recovery in each run is also shown in the table. Generally, the $\mathrm{N}_{2}$ recovery increased with an increase in the feed flov rate. The data and the simulation results for feed flow variation studies are shown in Figure 4-7, whereas those for sweep flow variation studies are shown in Figures 4-8 and 4-9. Effective membrane thickness used for simulation is 0.00485 inch. Better performance in permeator \#8 compared to permeator \#6 in terms of product quality was mainly due to the lower membrane thickness.

Water as Liquid Membrane and $40 \% \mathrm{CO}_{2}-60 \% \mathrm{CH}_{4}$ as Feed Gas

Separation studies with $\mathrm{CO}_{2}-\mathrm{N}_{2}$ feed mixture and He sweep gas in permeator \#6 (Table 4-14) showed that the helium content in purified feed gas was drastically reduced when the feed pressure was 45 psig. This implies that air can be used as sweep gas in the actual separation of a $\mathrm{CH}_{4}-\mathrm{CO}_{2}$ mixture. It is clear that nitrogen and oxygen concentration in the purified biogas mixture would be very low. With that objective, a 90-hour purification run with a $40 \%$ $\mathrm{CO}_{2}-60 \% \mathrm{CH}_{4}$ feed was carried out, with water as membrane in permeator \#6 and permeator \#7 connected in series. Pure nitrogen gas was used as a sweep. Both gas streams entering the permeator were completely dry. The flow rates of feed 


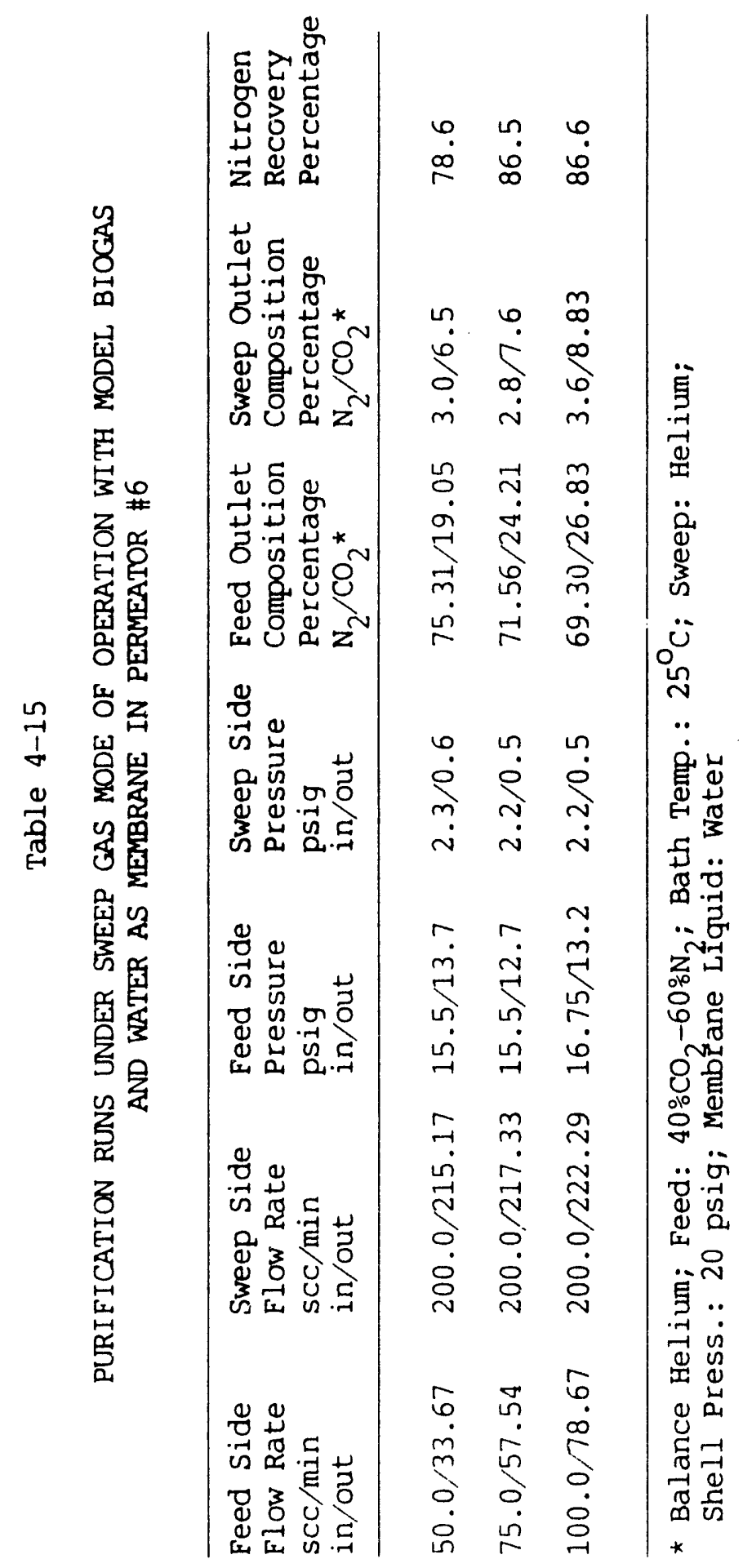



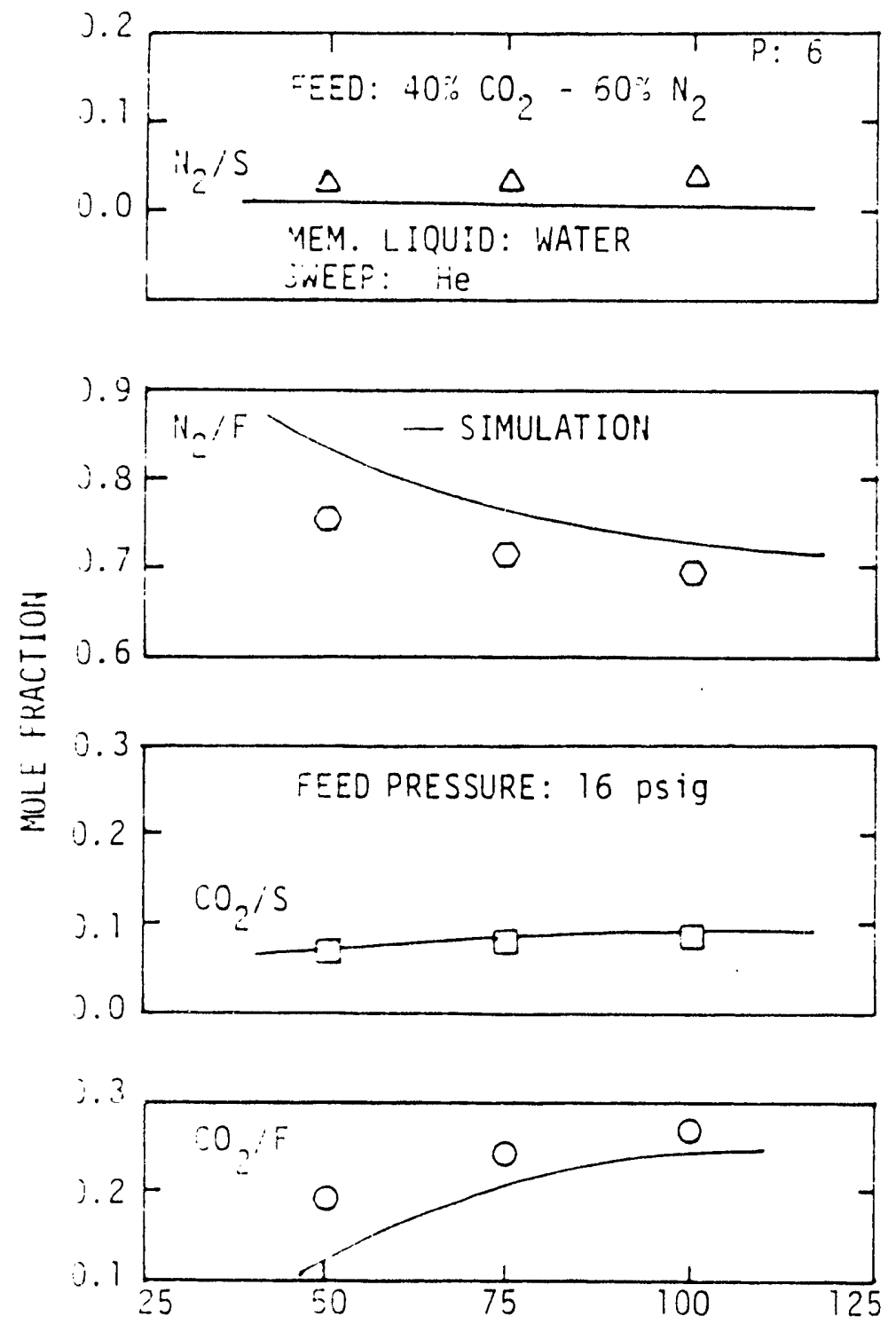

FEED FLOW RATE SCC/MIN

FIGURE $4-6$

EEED FLOW VARIATION STUDIES:

SWEEP GAS MODE OF OPERATION IN PERMEATOR \#6 


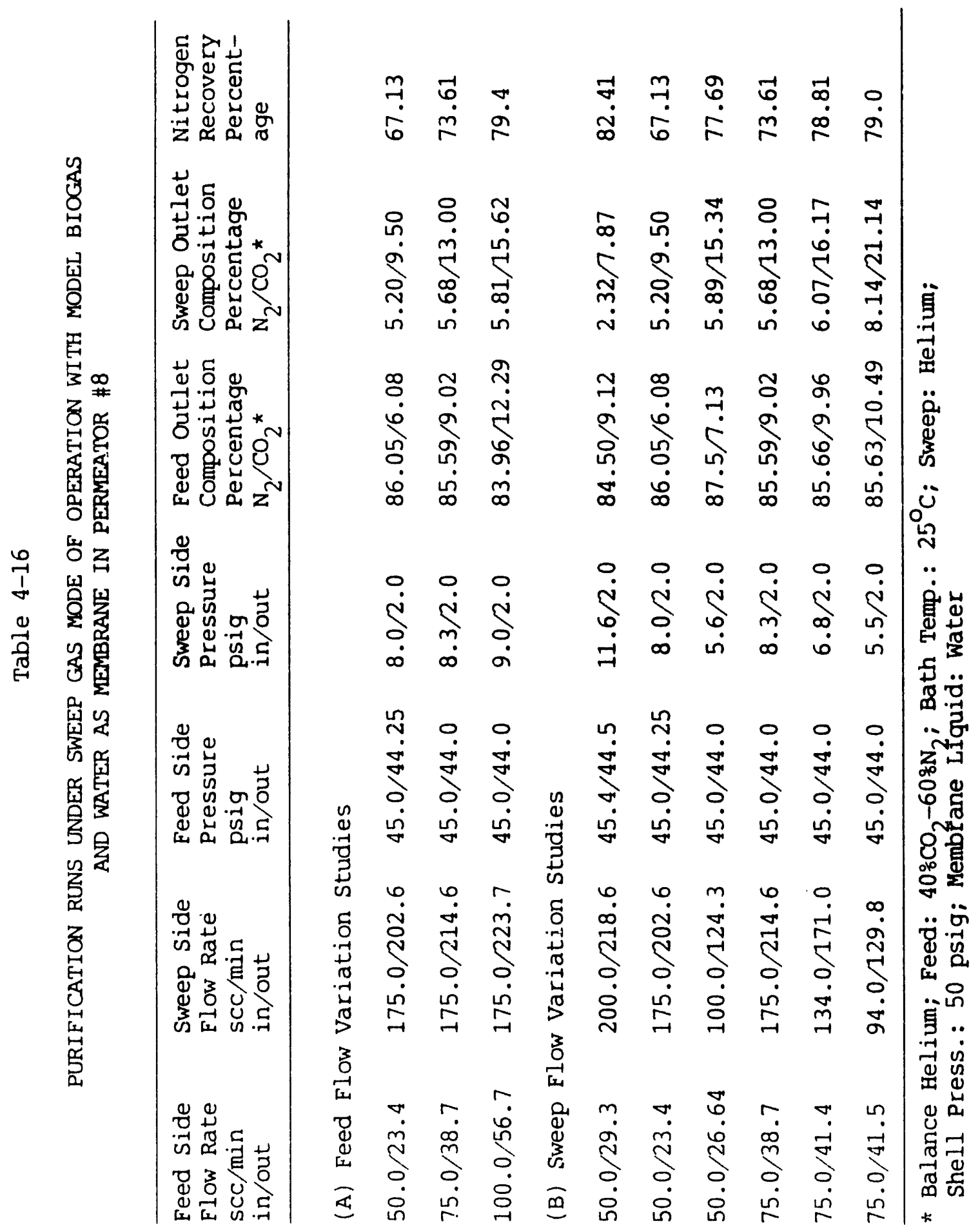




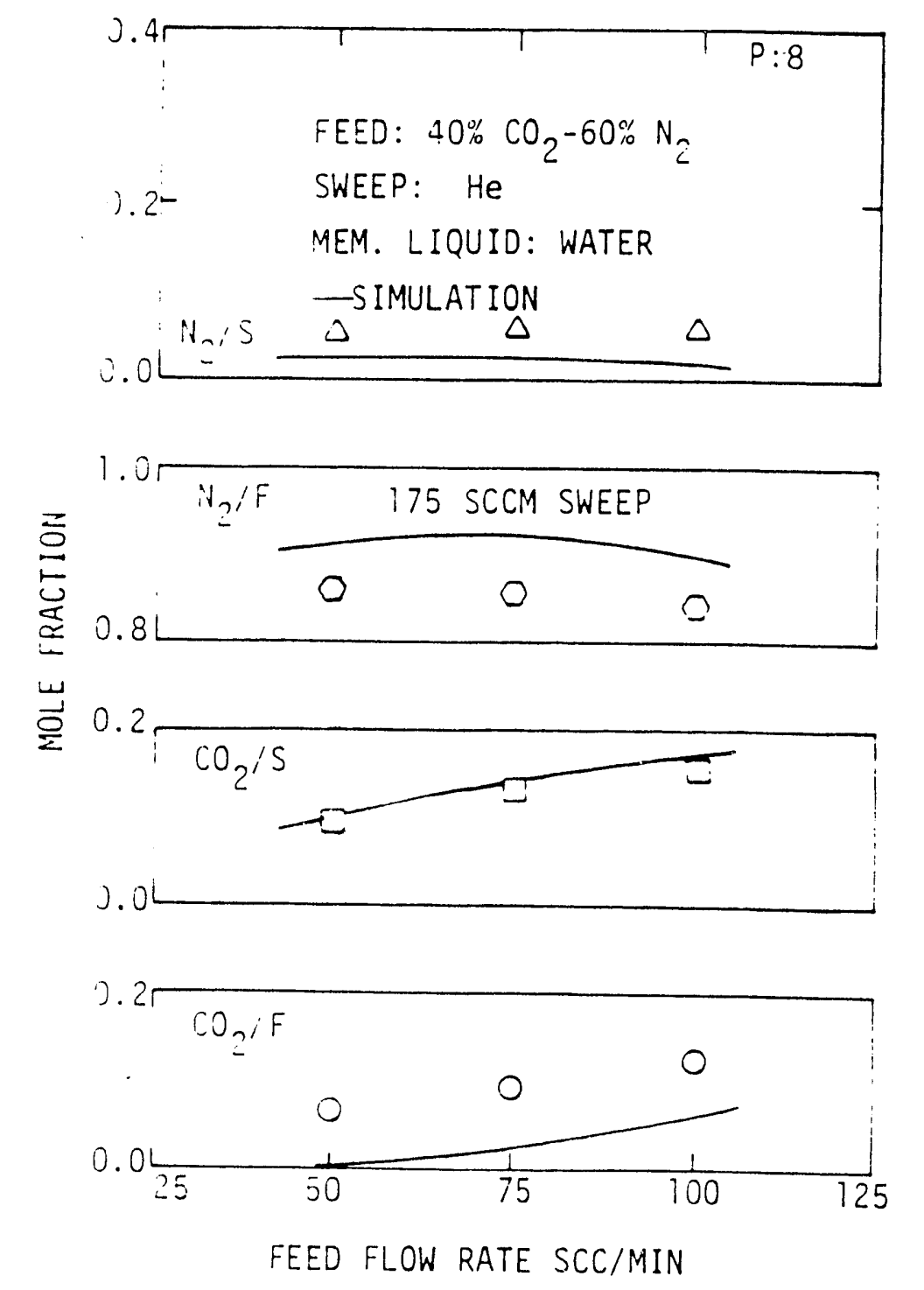

\author{
FIGURE $4-7$ \\ FEED FLOW VARIATION STUDIES: \\ SWEEP GAS MODE OF OPERATION IN PERMEATOR \# 8
}



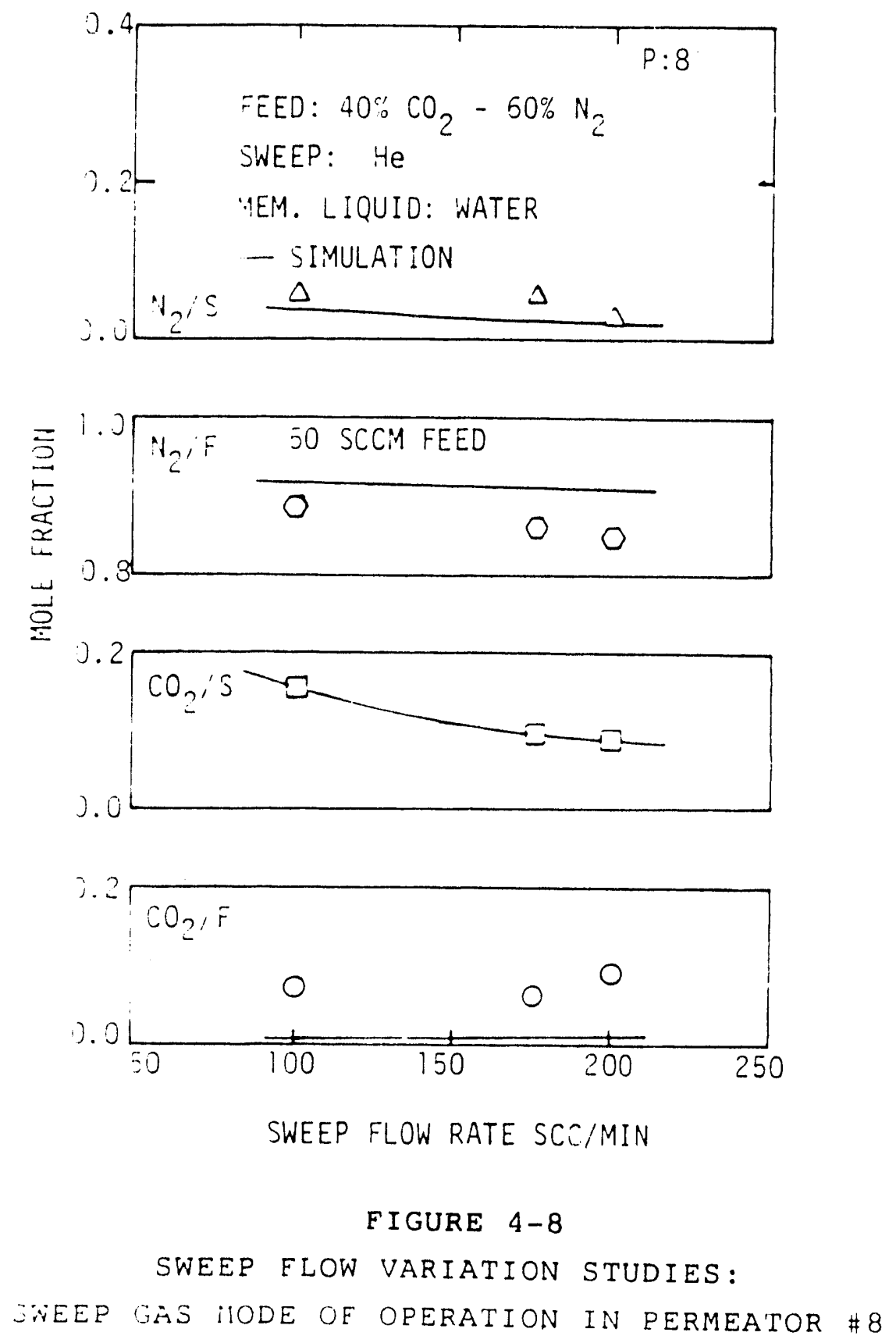


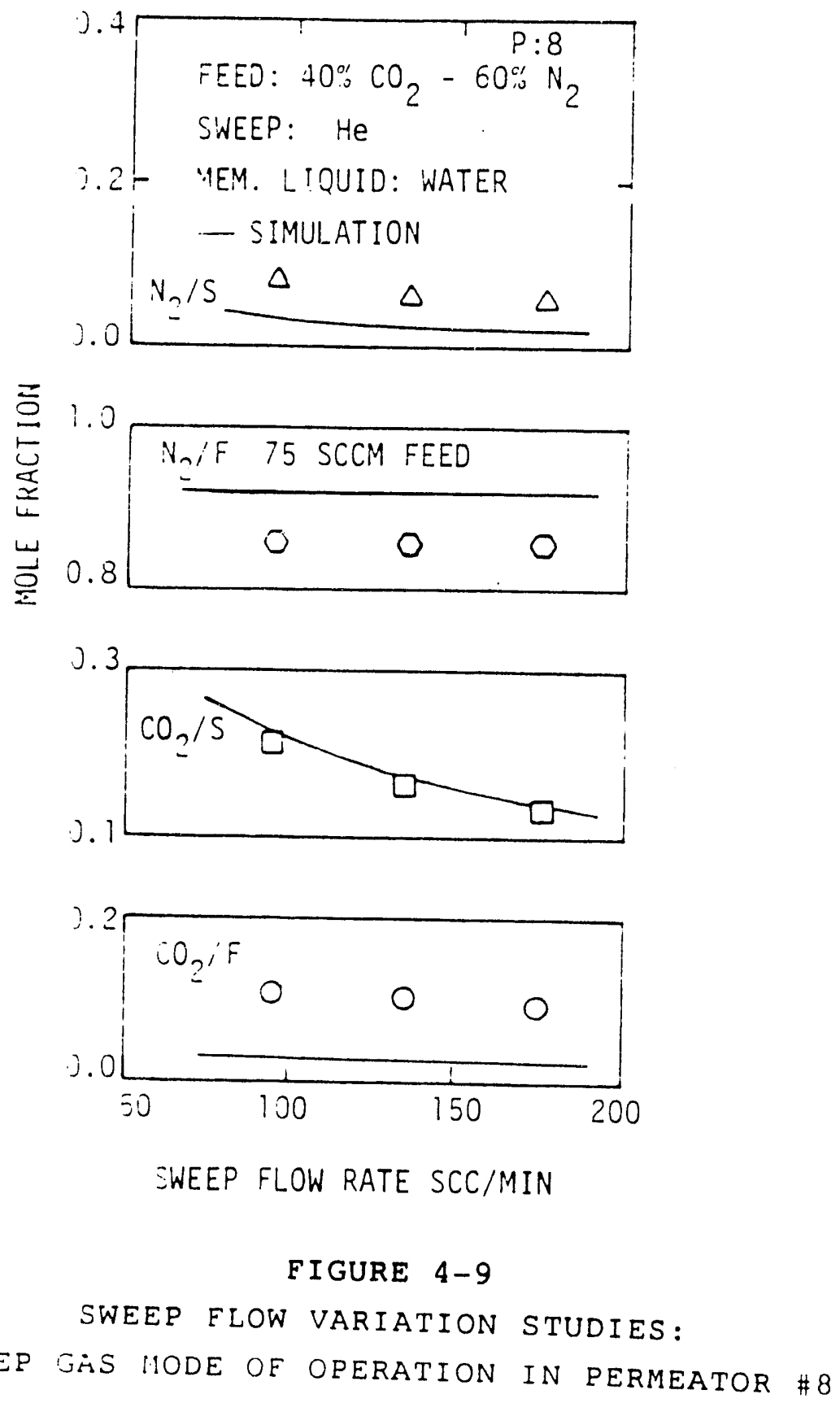


and sweep gas streams were $50 \mathrm{sccm}$ and $350 \mathrm{sccm}$, respectively. The shell side water membrane was pressurized by a helium gas cylinder. The results of this experiment are presented in Table 4-17. Steady performance was achieved with an average $\mathrm{CH}_{4}$ recovery of $87 \%$ in the purified biogas. Note that the purified gas stream contained only $5.5 \% \mathrm{CO}_{2}$. The permeation rate of sweep $\mathrm{N}_{2}$ into the feed gas was very low due to the much lower pressure drop in the large bore sweep fibers. The $\mathrm{N}_{2}$ content in the purified gas stream was only 2.5\%. Figure 4-10 graphically shows the membrane stability in this long-term run.

These results demonstrate that air can be used as sweep gas in actual operation. If air $\left(80 \% \mathrm{~N}_{2}-20 \% \mathrm{O}_{2}\right)$ were used instead of $\mathrm{N}_{2}$ as sweep gas, the $\mathrm{O}_{2}$ composition in the purified gas stream would have been close to $0.5 \%$. Further this run shows that the pressure drop in the sweep gas is only around 5 psi. This has to be compared with the 17 - 30 psi pressure drop encountered earlier with permeators containing smaller diameter fibers (NYSERDA Report 87-10). It is possible to make additional changes in such a mode of operation with separate sweep gas streams entering each permeator. This will reduce the sweep pressure drop to half the level observed here. That will reduce the $\mathrm{N}_{2}$ permeation rate and $\mathrm{N}_{2}$ content of the purified biogas still further. In fact, such a mode of operation may even be feasible with the smaller diameter fibers.

Table 4-18 provides the steady state data taken with feed and sweep flow rates maintained at $50 \mathrm{sccm}$ and $200 \mathrm{sccm}$, respectively using permeator \#8. Nitrogen gas was used as sweep. Simulation results based on the permeabilty of $\mathrm{CO}_{2}, \mathrm{CH}_{4}$ and $\mathrm{N}_{2}$ through water are also presented in the same table. The simulation describes the data satisfactorily. Note that, the result can be compared with the corresponding run for $\mathrm{CO}_{2}-\mathrm{N}_{2}$ feed gas mixture and helium sweep gas (4th row, Table 4-16), provided the pemeability of the gases are taken into consideration. As expected, recovery of $\mathrm{N}_{2}$ is more compared to $\mathrm{CH}_{4}$ as the latter is more permeable through water. On the other hand sweep helium being more permeable than sweep $\mathrm{N}_{2}$ results in a higher composition of $\mathrm{CH}_{4}$ compared to that of $\mathrm{N}_{2}$ in the purified gas.

$30 \mathrm{wt} \% \mathrm{R}_{2} \mathrm{CO}_{3}$ Solution as Liquid Membrane and $40 \% \mathrm{CO}_{2}-60 \% \mathrm{~N}_{2}$ as Peed Gas Separation runs of model biogas mixture $\left(40 \% \mathrm{CO}_{2}-60 \% \mathrm{~N}_{2}\right)$ with $30 \% \mathrm{~K}_{2} \mathrm{CO}_{3}$ 


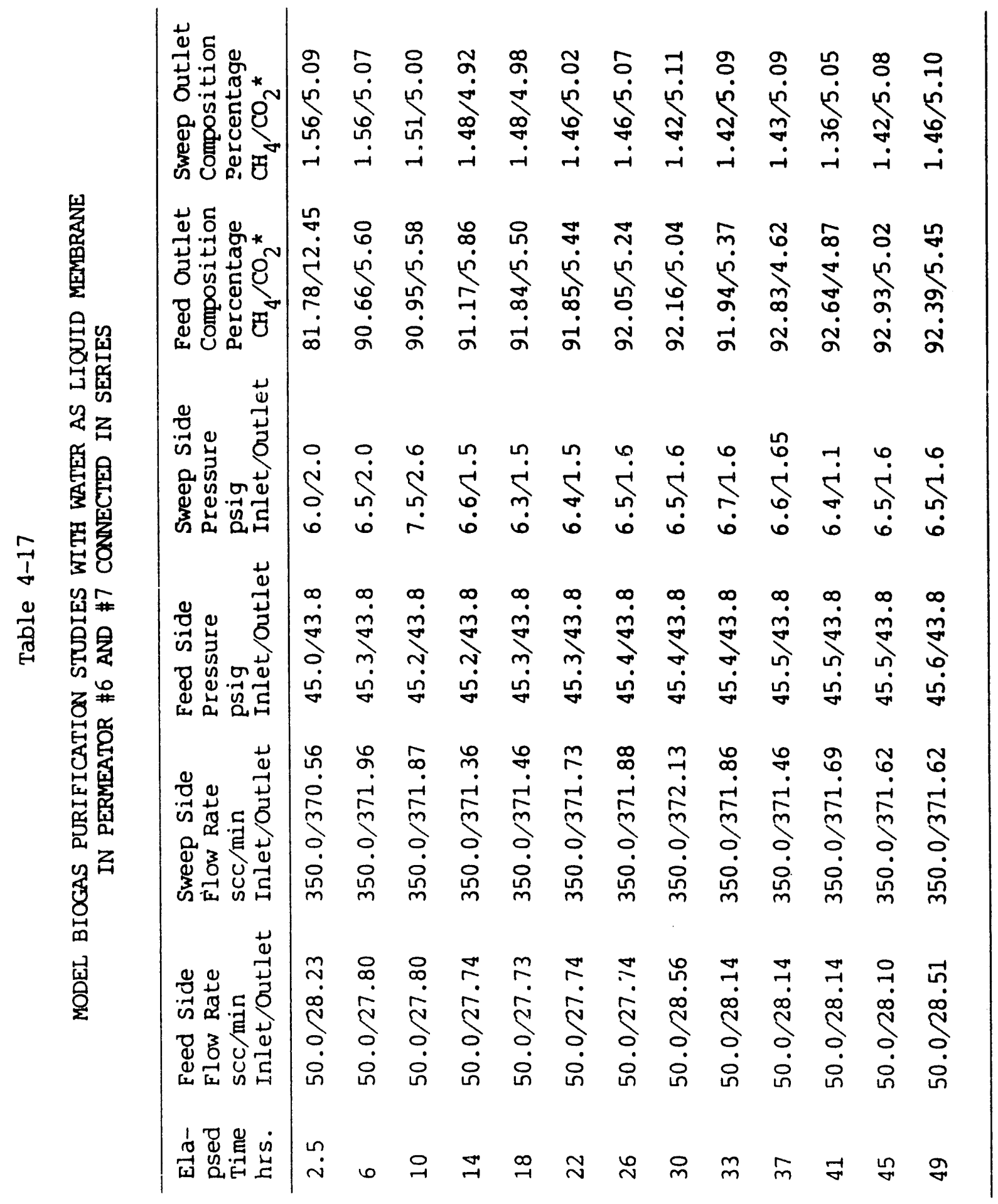




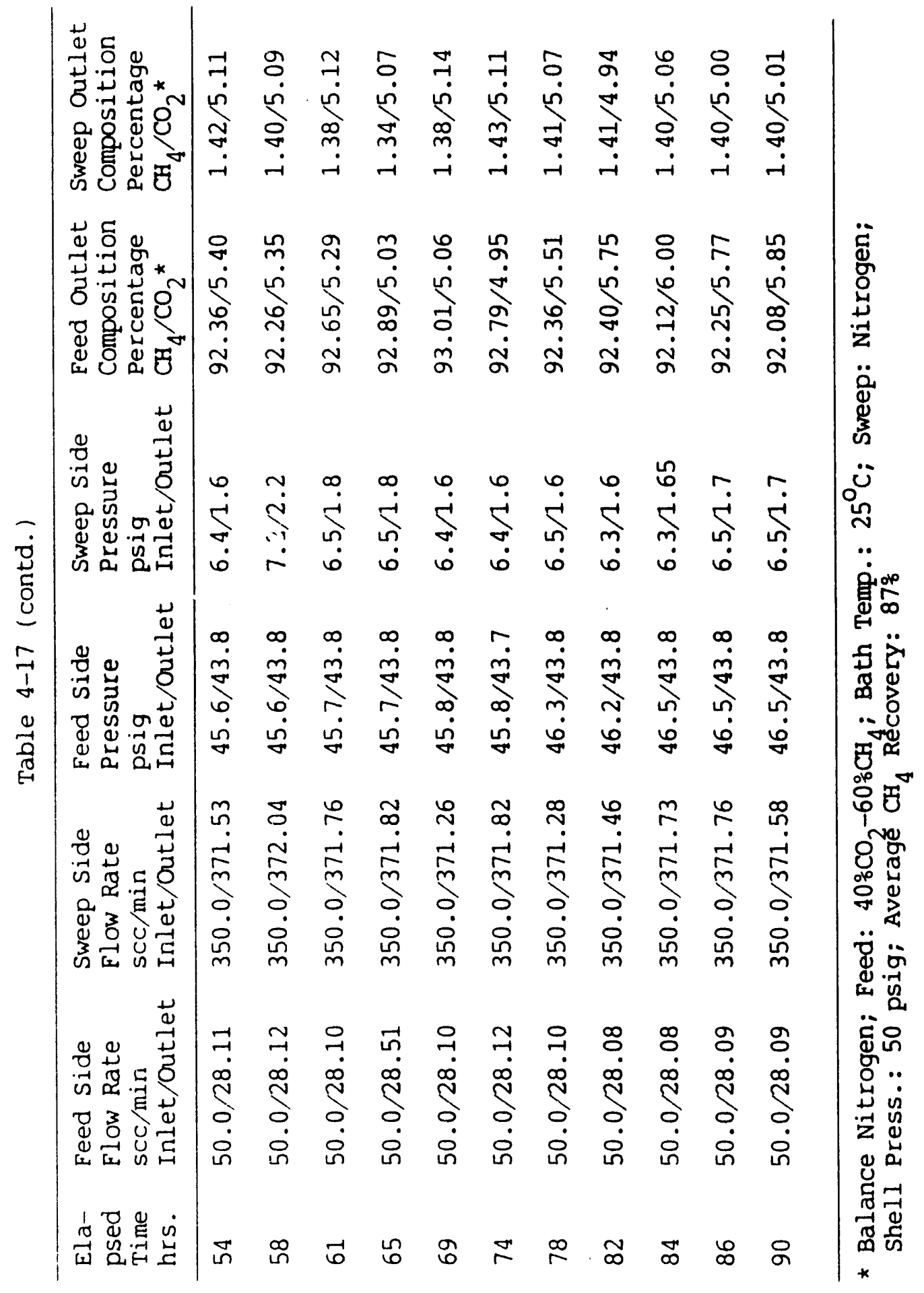


0.4

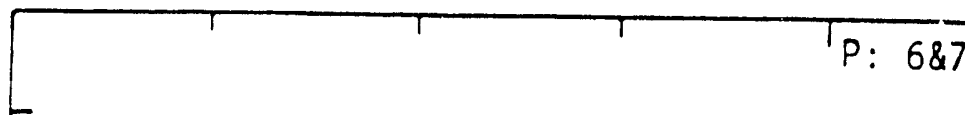

50 SCCM FEED - 350 SCCM SWEEP

$0.2 L$ FEED: $40 \% \mathrm{CO}_{2}-60 \% \mathrm{CH}_{4}$, SWEEP: $\mathrm{N}_{2}$ MEMBRANE LIQUID: WATER

$\mathrm{CH}_{4} / \mathrm{S}$ TEMPERATURE: $25^{\circ} \mathrm{C}$

$0.0-\Delta-\Delta-\hat{\Delta} \Delta \Delta \Delta-\Delta \Delta \Delta \sim \Delta \Delta \sim \Delta \sim \Delta \Delta \Delta \Delta$ i
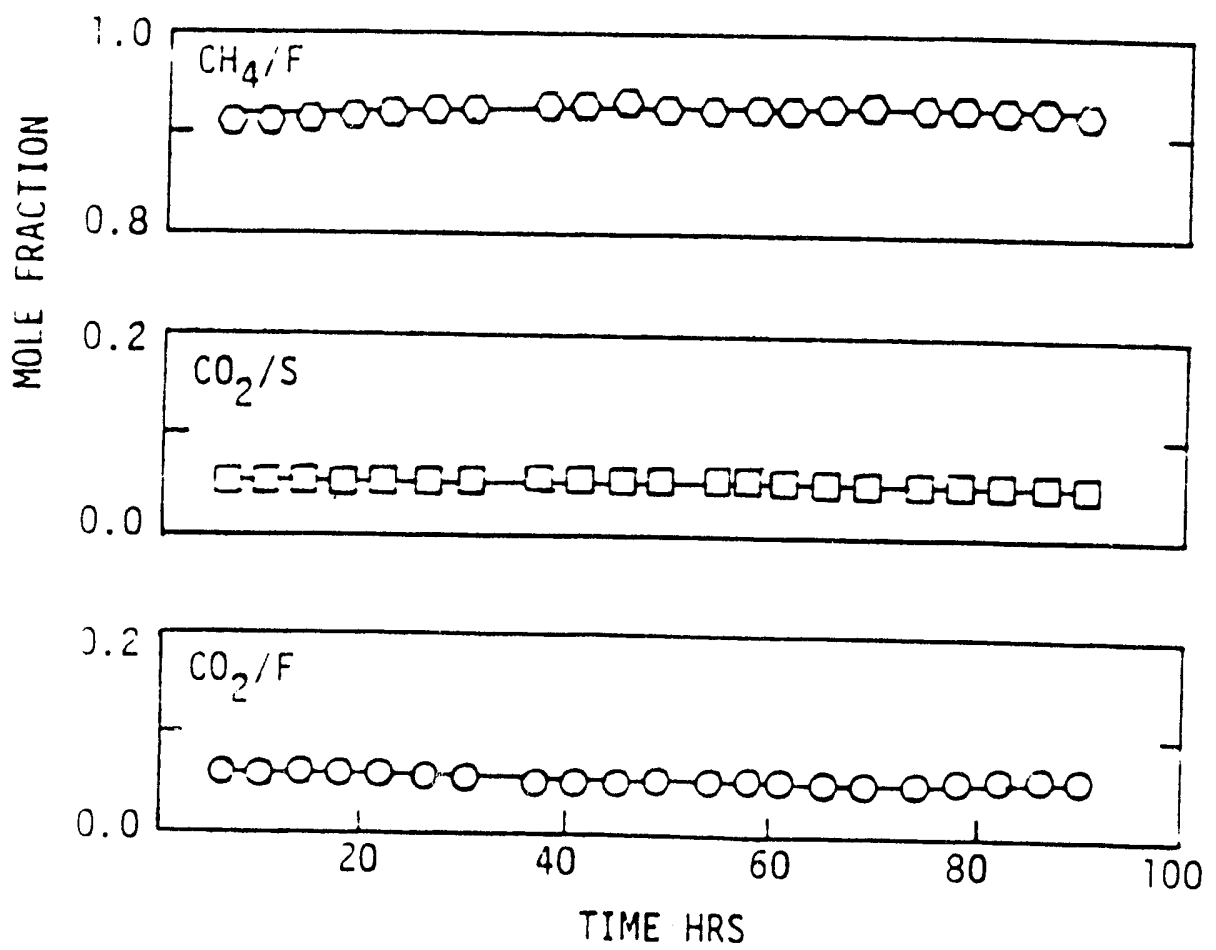

FIGURE $4-10$

CLM STABILITY STUDIES: SWEEP GAS MODE OF OPERATION WITH WATER AS MEMBRANE USING PERMEATORS \#6 AND \#7 CONNECTED IN SERIES 


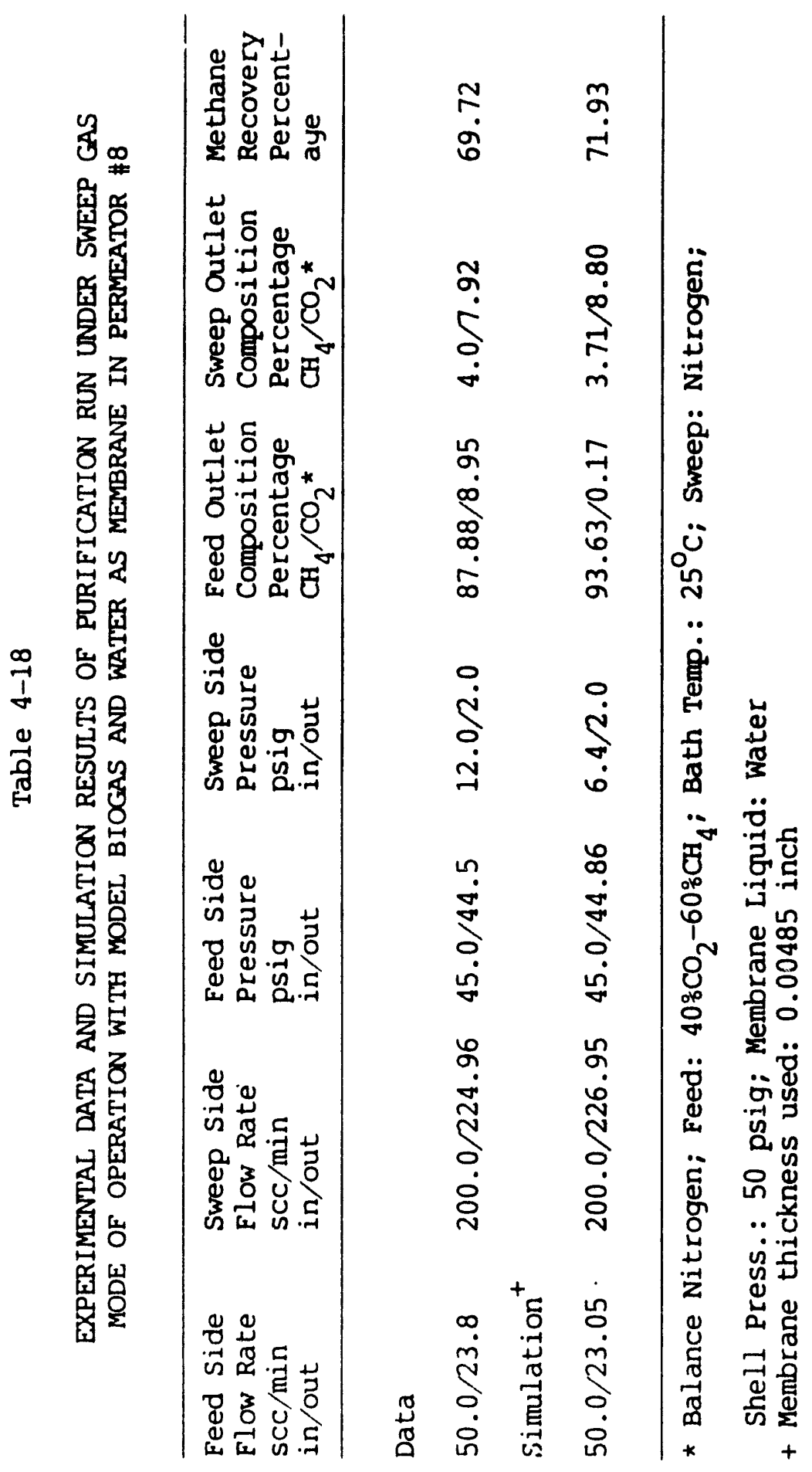


solution as membrane were carried out in permeator \#5. Table 4-19 shows steady state data from four different runs. Feed pressure was maintained at 30 psig for the first two runs; it is expected that, at the lower pressure, facilitation would be higher. The first data set indicated for $50 \mathrm{sccm}$ feed was obtained by running the system for 24 hours. The compositions, flow rates and pressures remained steady and stable. The same run was continued for another 30 hours with the feed flow rate changed to $100 \mathrm{sccm}$. Performance was stable in all respects in both the cases; $\mathrm{N}_{2}$ recoveries were also very high. However, the percentage of $\mathrm{CO}_{2}$ in the feed outlet stream was not that low. This can be due to lower permeability of $\mathrm{CO}_{2}$ in the $30 \% \mathrm{~K}_{2} \mathrm{CO}_{3}$ solution at $25^{\circ} \mathrm{C}$ and also possibly due to the lower feed pressure.

The previous run was then continued by increasing the feed inlet pressure to 45 psig. Feed and sweep gas flow rates were adjusted to $50 \mathrm{sccm}$ and $350 \mathrm{sccm}$, respectively. The run was continued for 24 hours but the $\mathrm{CO}_{2}$ removal efficiency did not change significantly. The same run was continued for another 30 hours by increasing the feed flow rate to $100 \mathrm{sccm}$. Surprisingly enough, the pressure remained steady but the performance deteriorated with respect to $\mathrm{CO}_{2}$ composition in the feed outlet. The steady state data, shown in Table 4-19 suggest that the mass transfer resistance increased with the progress of the run. This may be attributed to condensation of water in the defective pores in the microporous wall of the fibers containing salt solution or some kind of deposition of fine salt particles which might have caused significant increase in the effective membrane thickness. In the traditional absorption process carried out below $40^{\circ} \mathrm{C}$, the solution strength is limited to about $12 \mathrm{wt} \% \mathrm{~K}_{2} \mathrm{CO}_{3}$ to avoid precipitation (Astarita et al., 1983). The numerical simulation results and the steady state values are also compared in the same table. Simulation results based on simple diffusion with no reaction predicts the data quite well. Simulation results based on other approximations ( 0 to and Quinn, 1971) predict a higher permeation rate of $\mathrm{CO}_{2}$.

In order to check whether there is really any fouling of the pores of the microporous hollow fibers, permeator \#5 was thoroughly cleaned after completion of the separation experiments with $\mathrm{K}_{2} \mathrm{CO}_{3}$ solution as the liquid membrane. The following cleaning procedure was adopted. Shell side was flushed with distilled water for a long period. Then shell side was filled with water and pressurized 


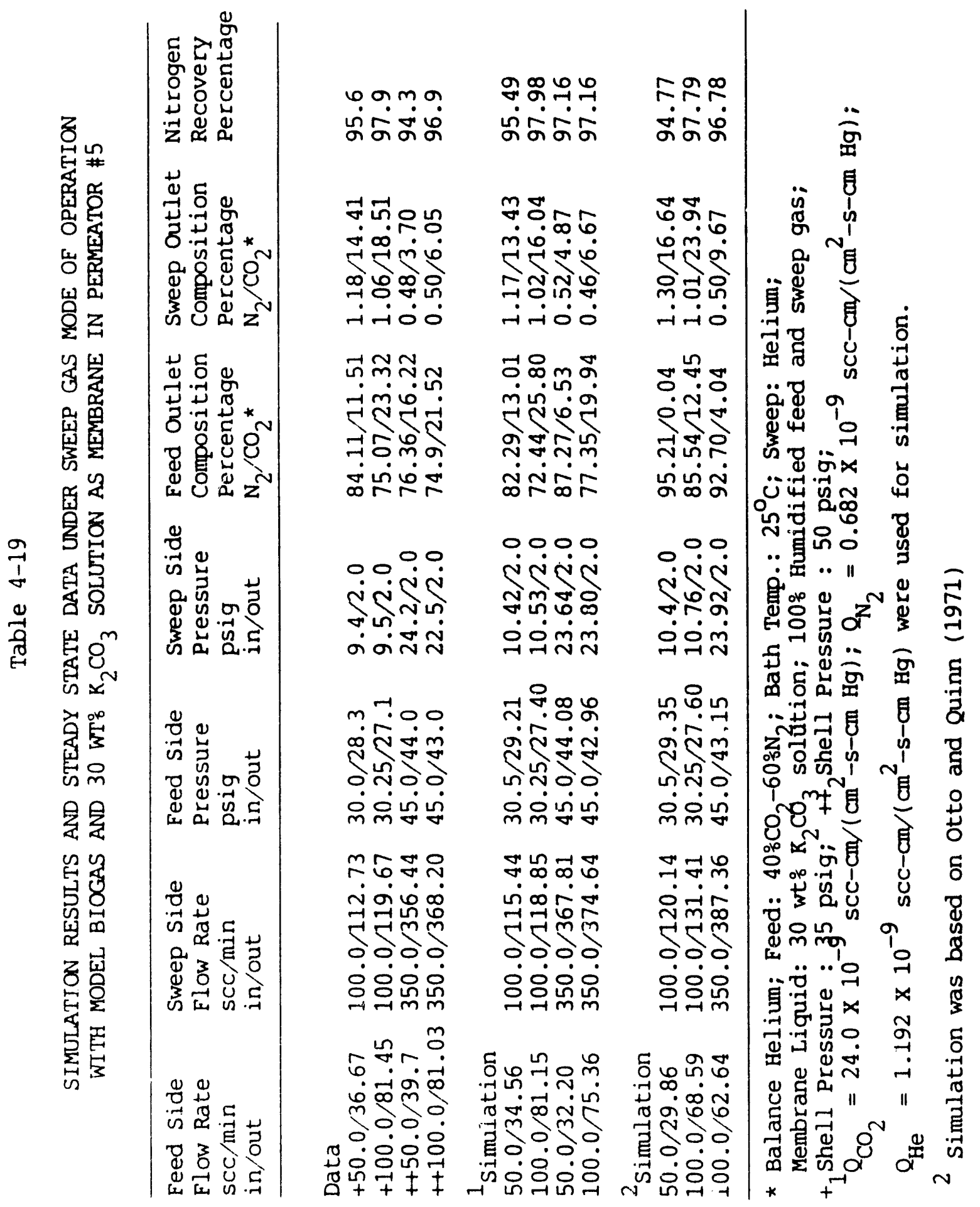


gradually upto 50 psig and water was passed through both the tube sides for 24 hrs. Finally the permeator was driad using $\mathrm{N}_{2}$ gas. Separation experiments were then again carried out in permeator \#5 with $\mathrm{CO}_{2}-\mathrm{N}_{2}$ feed gas and He sweep gas having pure water as a liquid membrane. The results are presented in Table 4-20 and they are quite different from those obtained earlier (see data in Table 4-11). The results suggest that there is some kind of irreversible fouling of the pores in the microporous hollow fibers by the fine crystals of $\mathrm{K}_{2} \mathrm{CO}_{3}$ salt which enhances the condensation in the pores, and thereby increases the membrane thickness.

\section{Aqueous Solution of DEA as Liquid Membrane and $40 \% \mathrm{CO}_{2}-60 \% \mathrm{~N}_{2}$ as Feed Gas}

Purification runs for model biogas mixture were also carried out with aqueous 10, 20 and 30 wt\% solutions of diethanolamine (DEA) as liquid membranes using permeator \#8. The steady state data for both feed and sweep flow variation studies with 10 wt\% DEA as membrane are shown in Table 4-21. Similarly the results for 20 and 30 wt\% DEA are shown in Tables 4-22 and 4-23, respectively. In all these studies, a gas mixture containing $40 \% \mathrm{CO}_{2}-60 \% \mathrm{~N}_{2}$ was used as feed and pure helium was introduced as sweep. Both the feed and sweep gases were completely humidified. Feed gas inlet pressure was maintained at 45 psig in all experiments except one run with $30 \mathrm{wt} \%$ DEA where it was maintained at 25 psig.

As expected, the $\mathrm{CO}_{2}$ percentage in the purified model biogas increased with an increase in feed gas flow rate for all experiments. However, for $10 \mathrm{wt} \%$ DEA as liquid membrane, an increase in the sweep flow rate for a certain feed flow rate of $100 \mathrm{sccm}$ did not improve the level of feed gas purifiction (Table 4-21), suggesting saturation of the carrier in the membrane: the downstream partial pressure of $\mathrm{CO}_{2}$ has very little effect.

Simulation results are also presented in respective tables. An average permeability of $\mathrm{CO}_{2}$ was determined corresponding to each run; a particular value, that predicted the compositions of feed and sweep outlet as well as nitrogen recovery well, was chosen. Highest separation factor $\left(Q_{\mathrm{CO}_{2}} / Q_{\mathrm{N}_{2}}\right)$ was obtained in the case of $30 \mathrm{wt} \%$ DEA; however, $\mathrm{CO}_{2}$ composition at feeci outlet was relatively high compared to the results obtained with $10 \mathrm{wt} \%$ and 20 wt\% DEA 


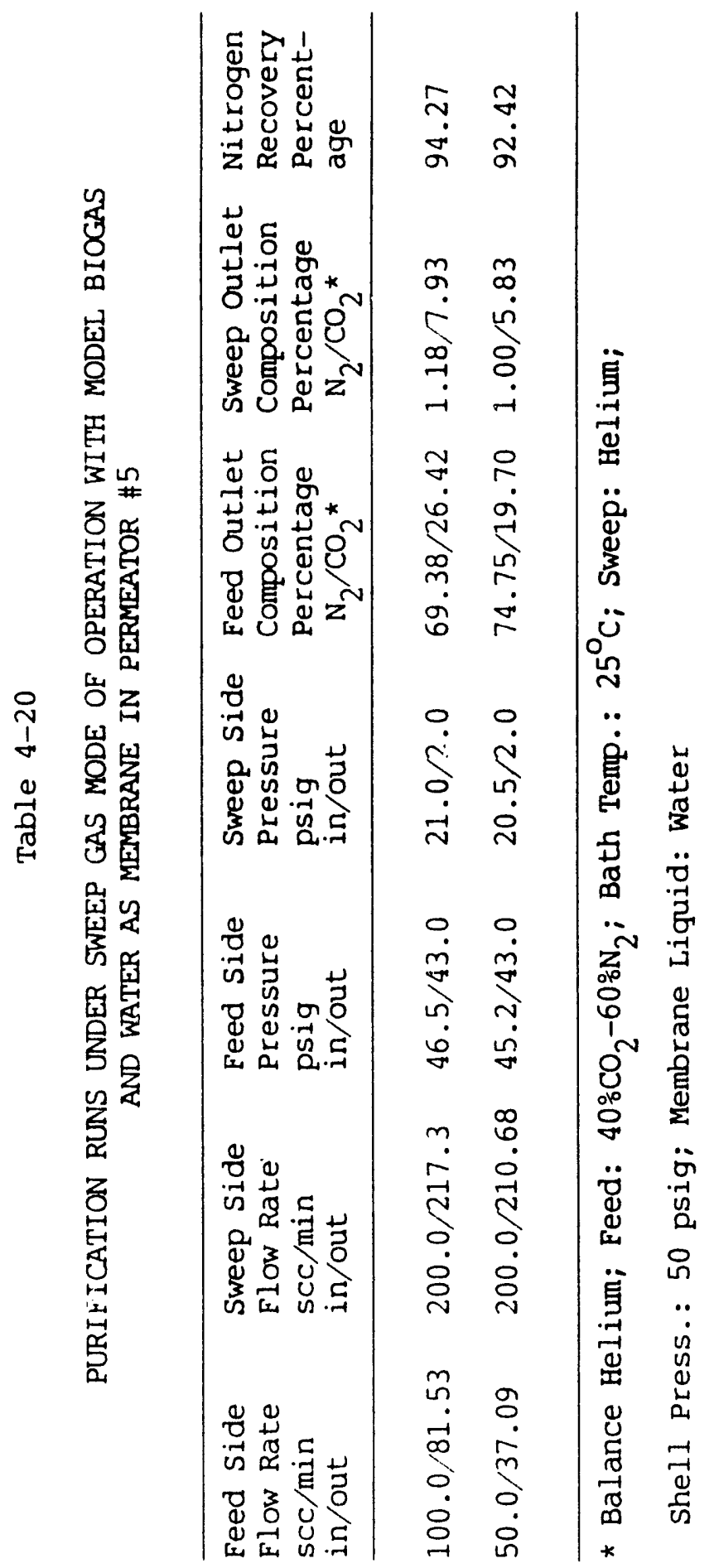




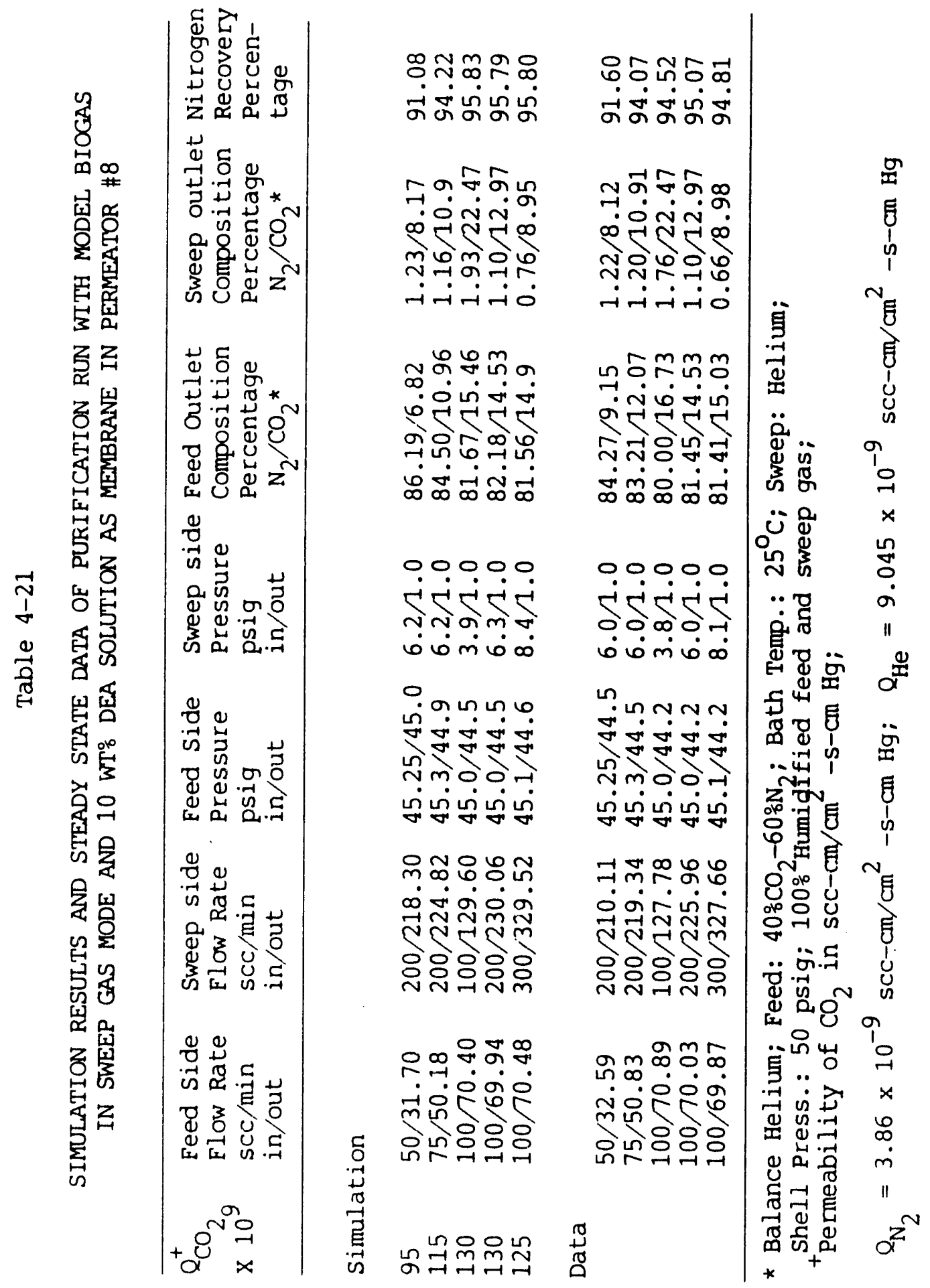




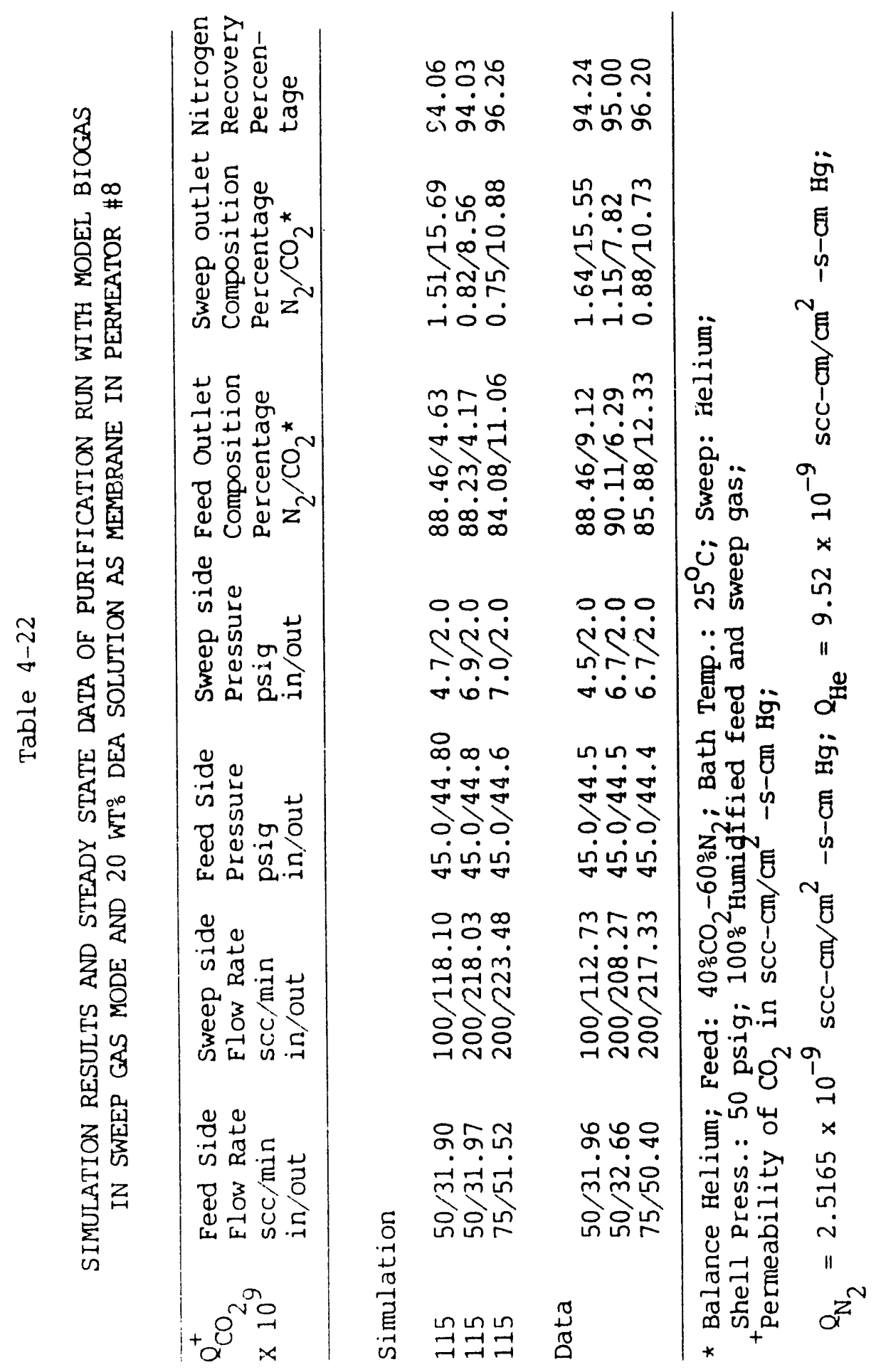




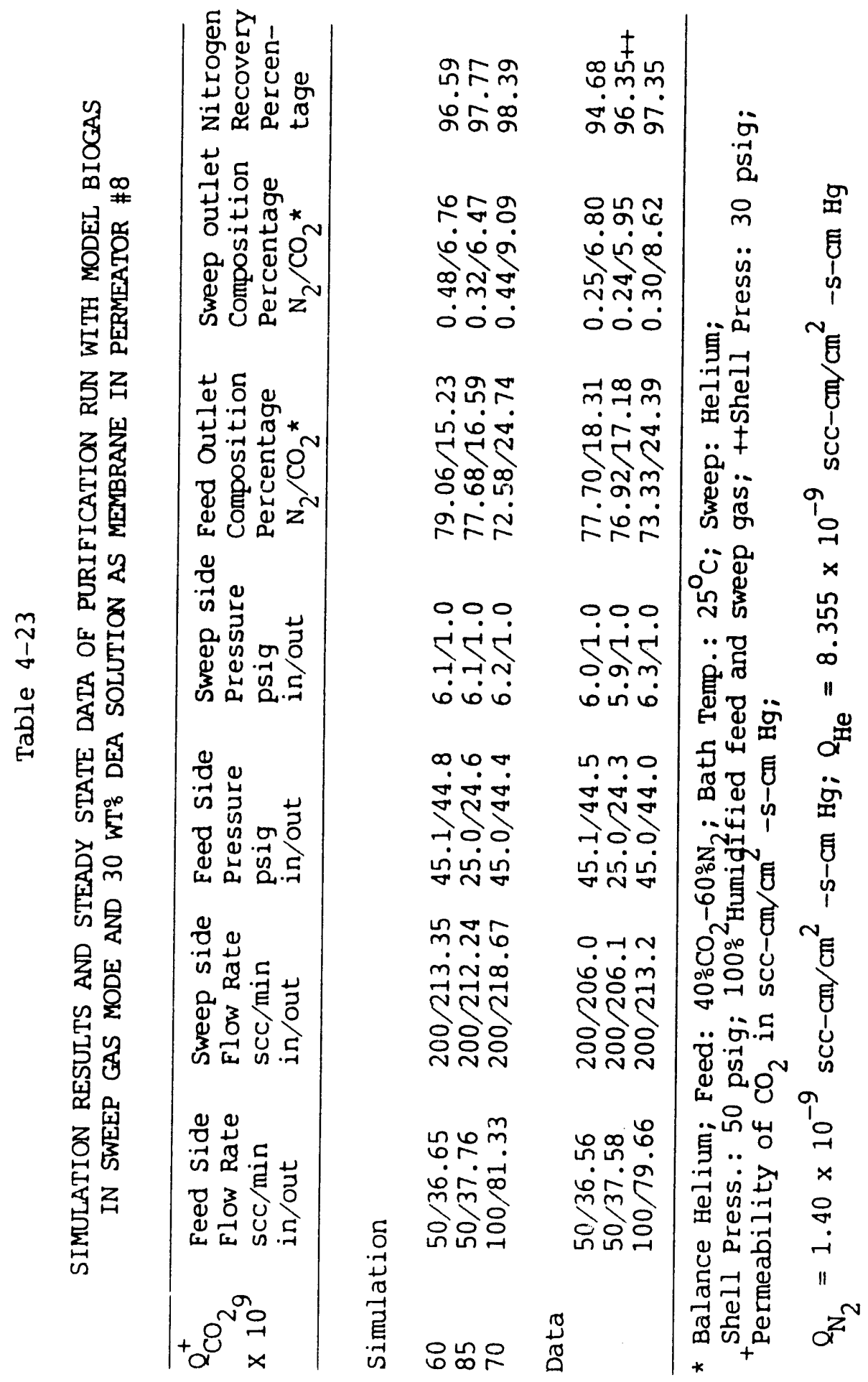


under identical conditions. Note that, with $30 \mathrm{wt} \%$ DEA as liquid membrane, the performance at low feed pressure was more or less similar to that obtained with the high pressure run (Table 4-23) even though the partial pressure driving force for $\mathrm{CO}_{2}$ was much less in the first case. This is due to higher facilitation of $\mathrm{CO}_{2}$, normally achieved at lower partial pressure of $\mathrm{CO}_{2}$. As expected nitrogen recovery was also slightly higher.

The solution having a composition of $20 \mathrm{wt} \%$ DEA was found to be the best liquid membrane both from the point of purity of the feed gas as well as recovery of nitrogen. Figure 4-11 shows the DEA concentration variation studies at a paricular feed and sweep gas flowrate. Quality of the purified gas as well as recovery of $\mathrm{N}_{2}$ improved as the concentration of DEA increased up to $20 \mathrm{wt}$. However, in spite of high recovery, the quality of the purified feed gas deteriorated drastically when the concentration of DEA was increased to $30 \mathrm{wt} \%$. This is most likely due to a change in the reaction kinetics at the higher amine concentrations (Blauwhoff et al., 1983). Smith and Quinn (1979) observed similar phenomena for facilitated transport of $\mathrm{CO}_{2}$ through MEA and DEA solutions. Much slower tranport rates at higher concentration as well as an apparent decrease in the reaction rate as all species concentration increases were also observed by Donaldson and Nguyen (1980).

Long-term stability runs for 10,20 and $30 \mathrm{wt} \%$ DEA solutions are shown in Tables 4-24, 4-25 and 4-26, respectively. Steady performance was obtained in all runs unless there was a change in the surrounding temperature. Variation in room temperature as well as bath temperature affected the performance which was expected as the reaction rates were temperature dependent. Figures 4-12, 4-13 and 4-14 show the plots of the above three stability runs.

To study the effect of temperature on model biogas separation, a long-term experiment was carried out at a higher temperature using aqueous $20 \mathrm{wt} \%$ DEA solution as the liquid membrane in permeator \#8. Generally, the rate of a reaction increases with an increase in temperature. It was speculated that better purification would be achieved as more $\mathrm{CO}_{2}$ would permeate from the feed to the sweep side due to an increase in the rate of reaction between $\mathrm{CO}_{2}$ and DEA. 


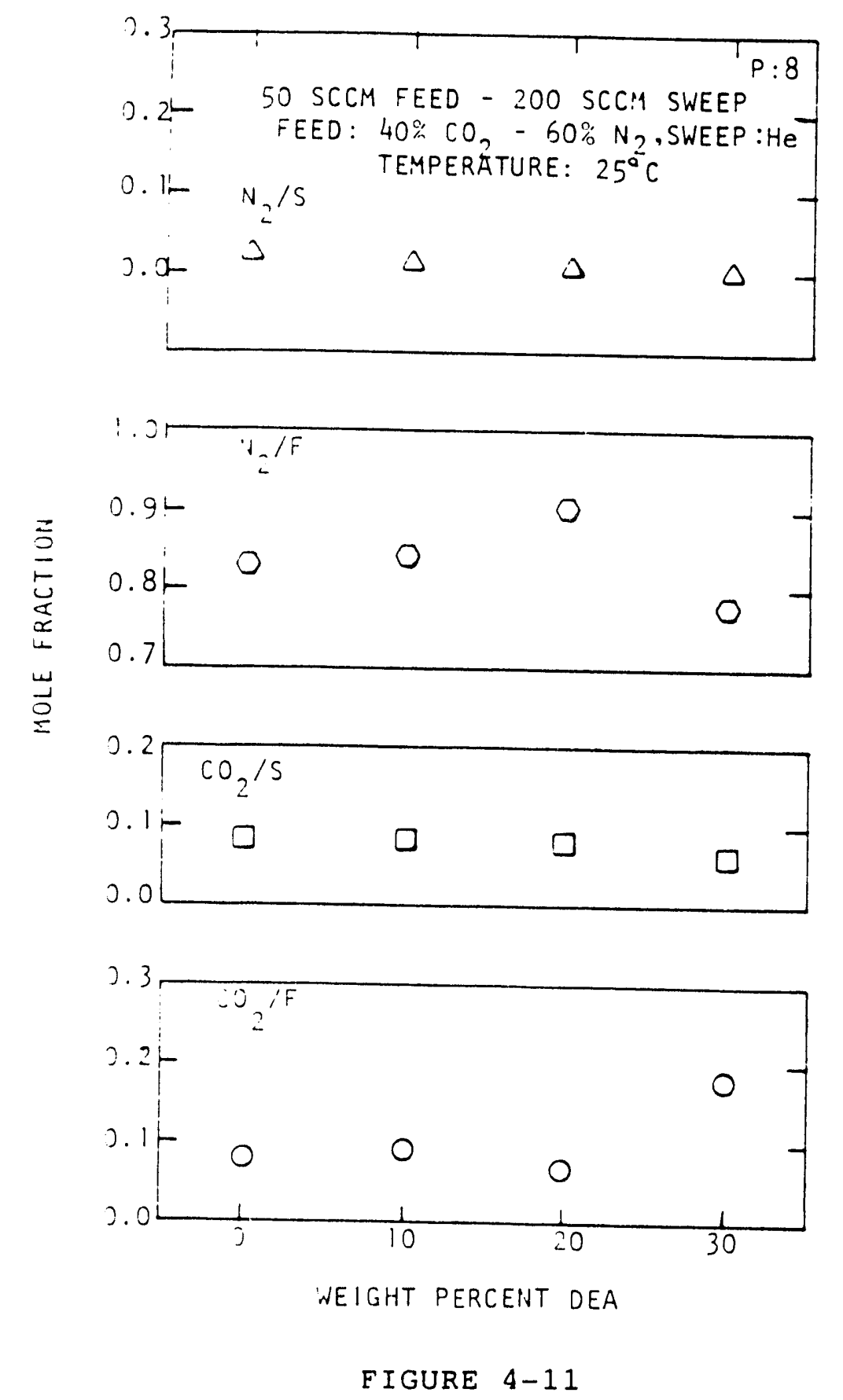

DEA CONCENTRATION VARIATION STUDIES:

SWEEP GAS MODE OF OPERATION IN PERMEATOR \# 8 


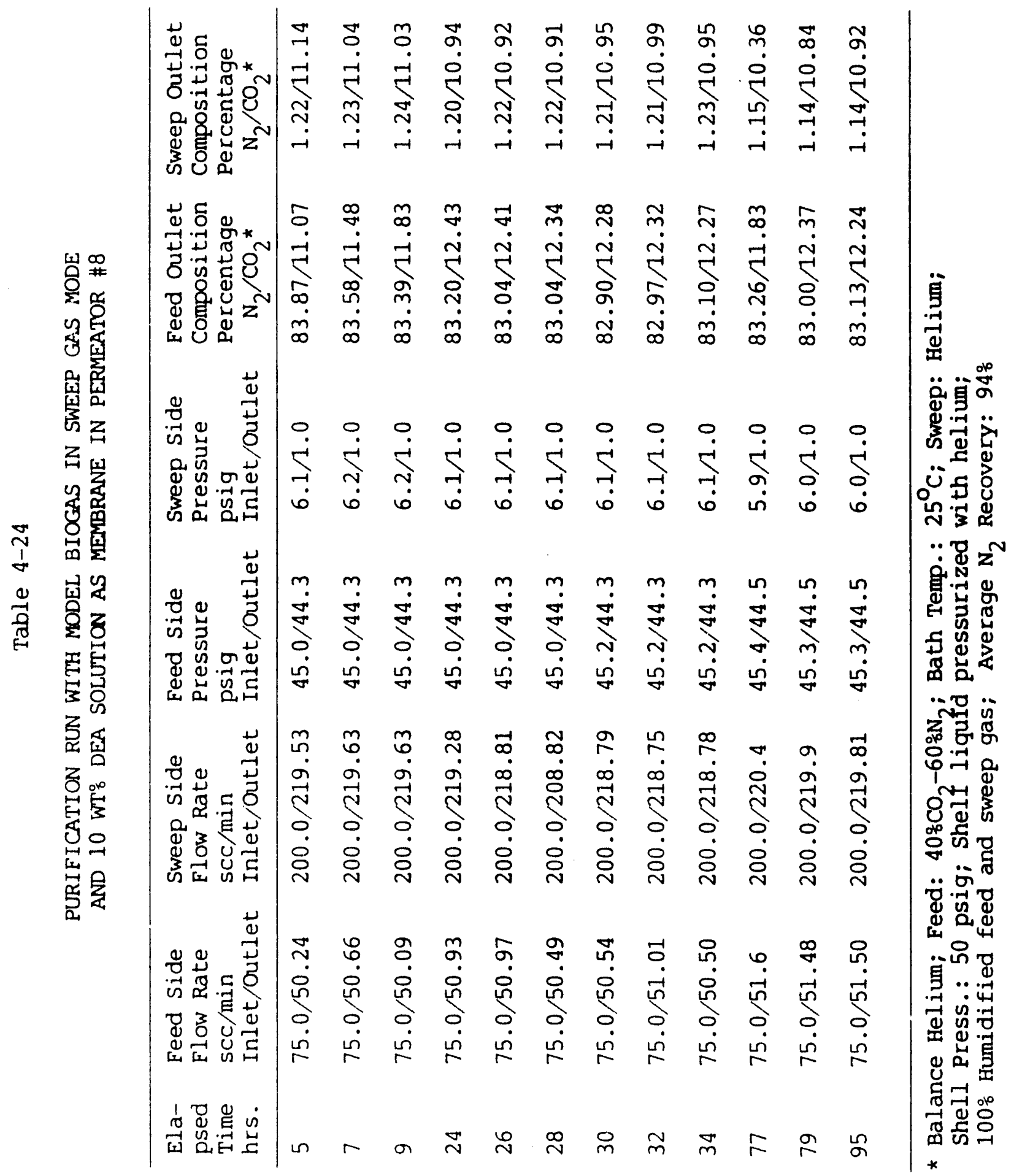




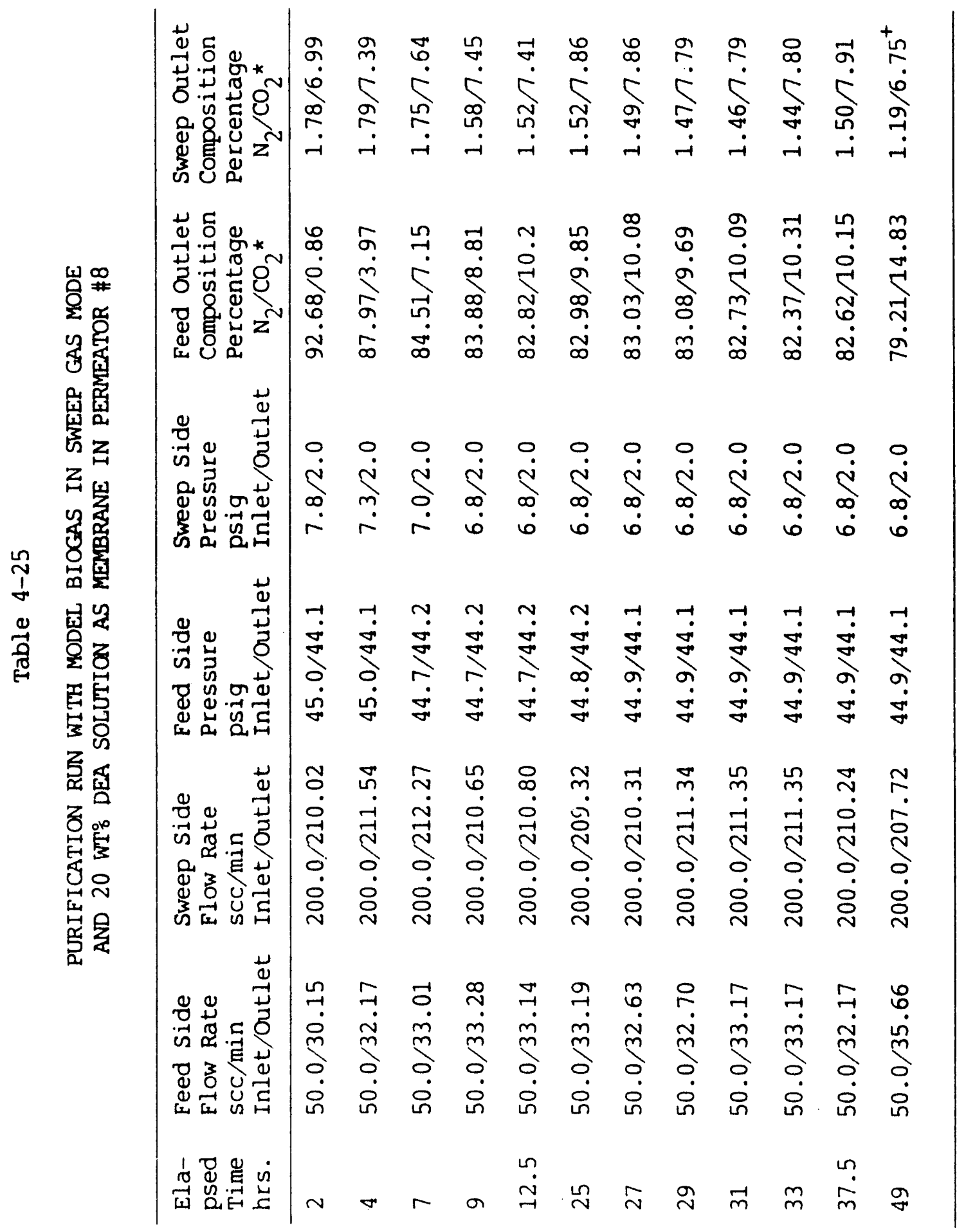




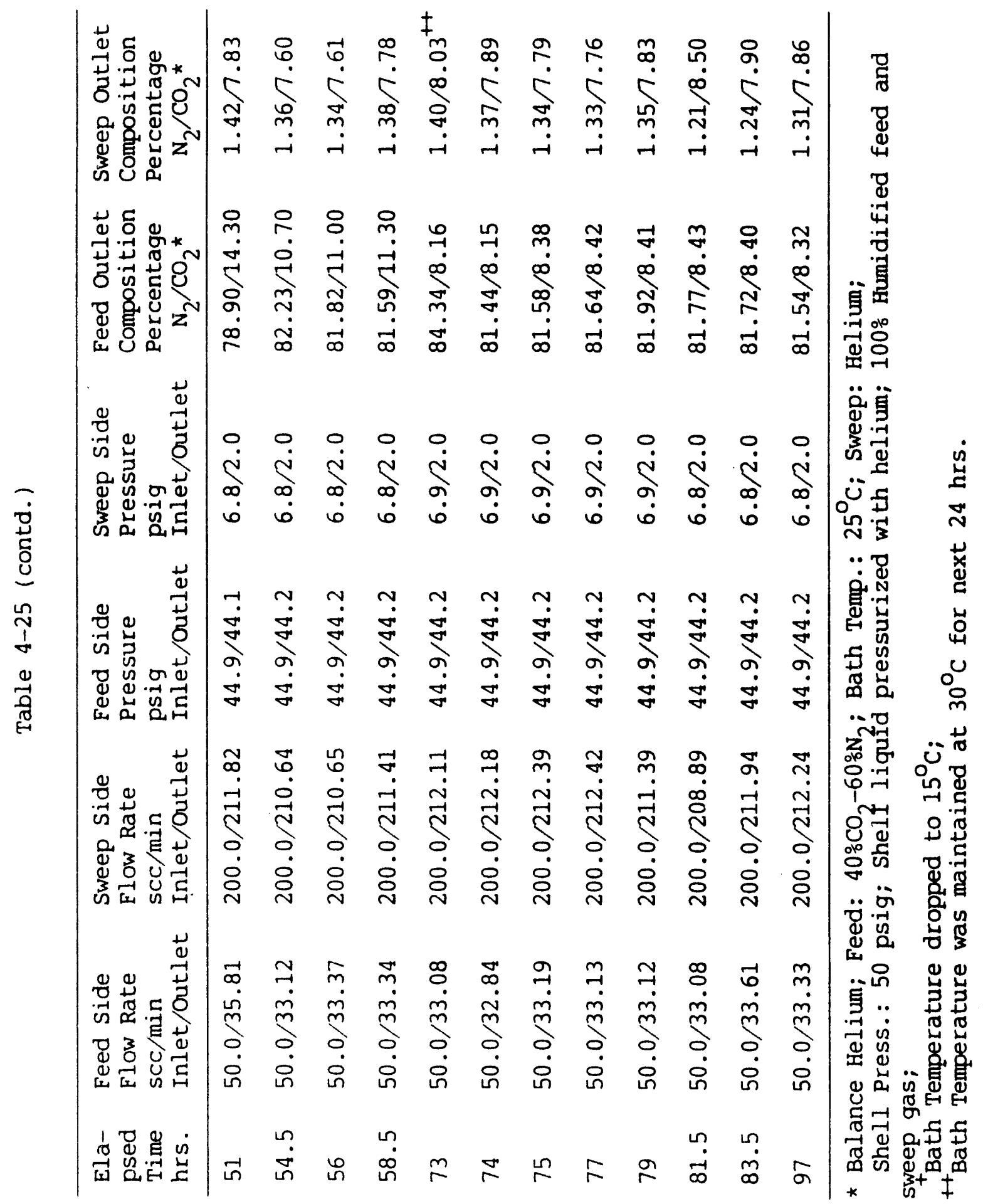




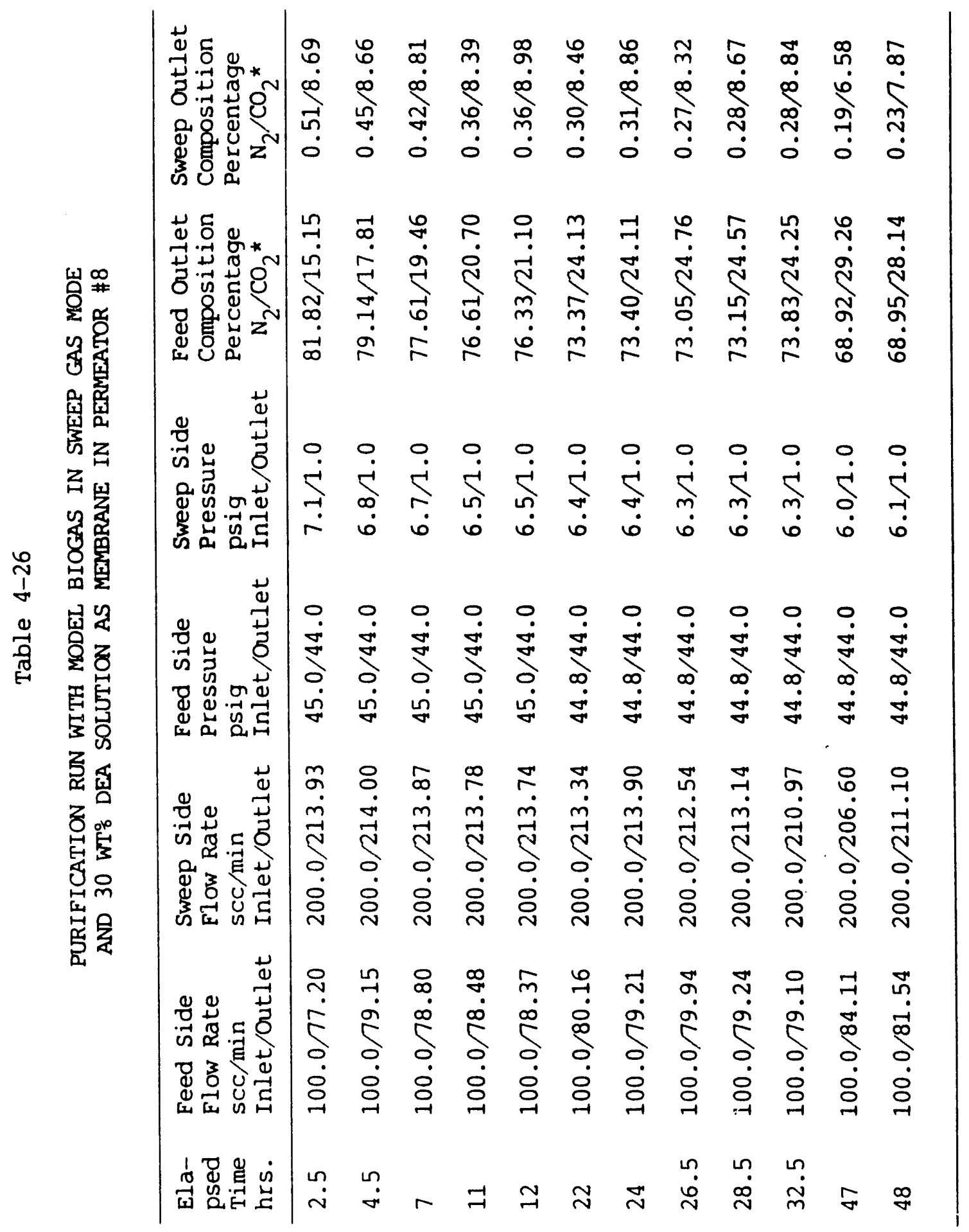




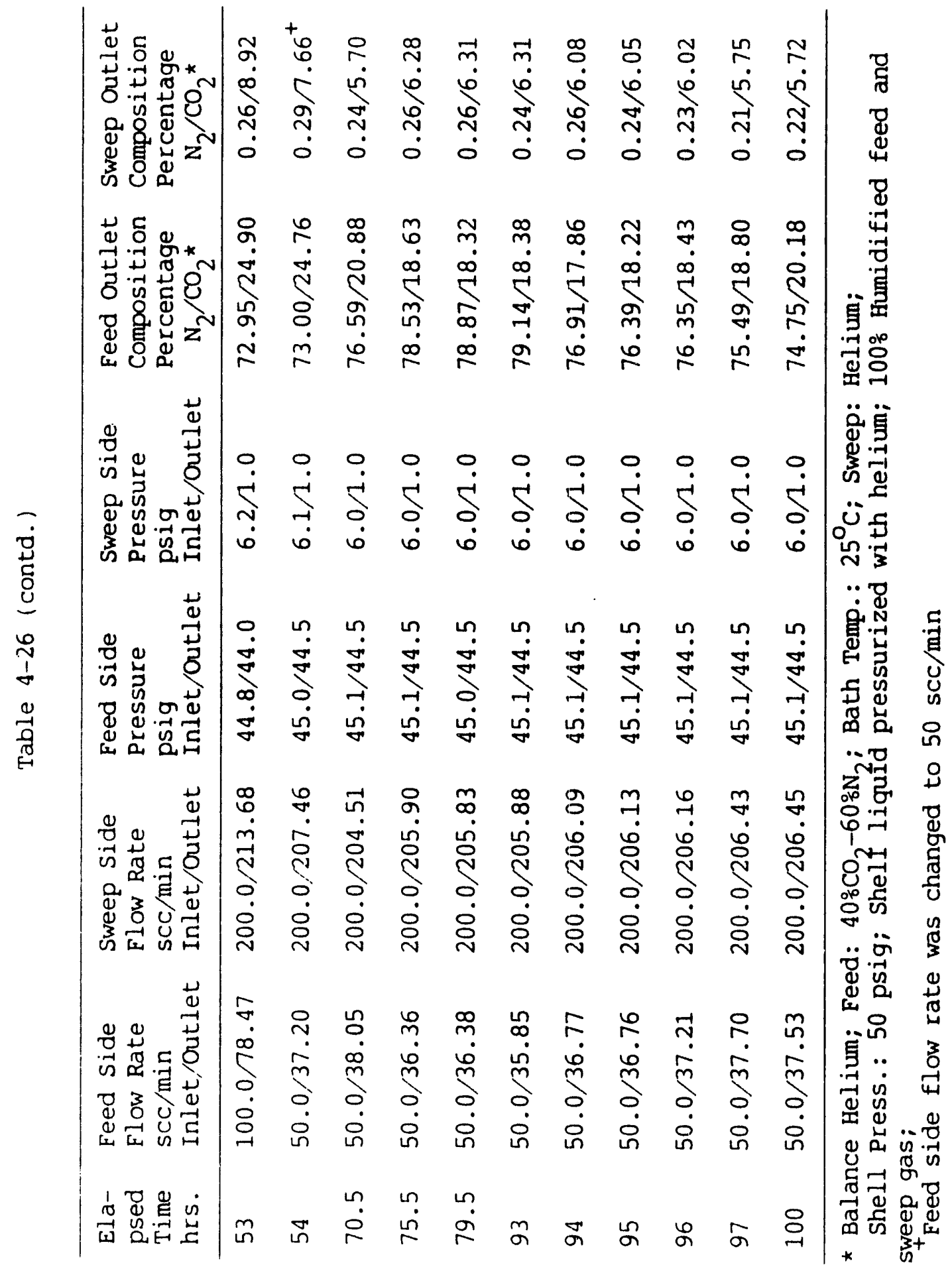



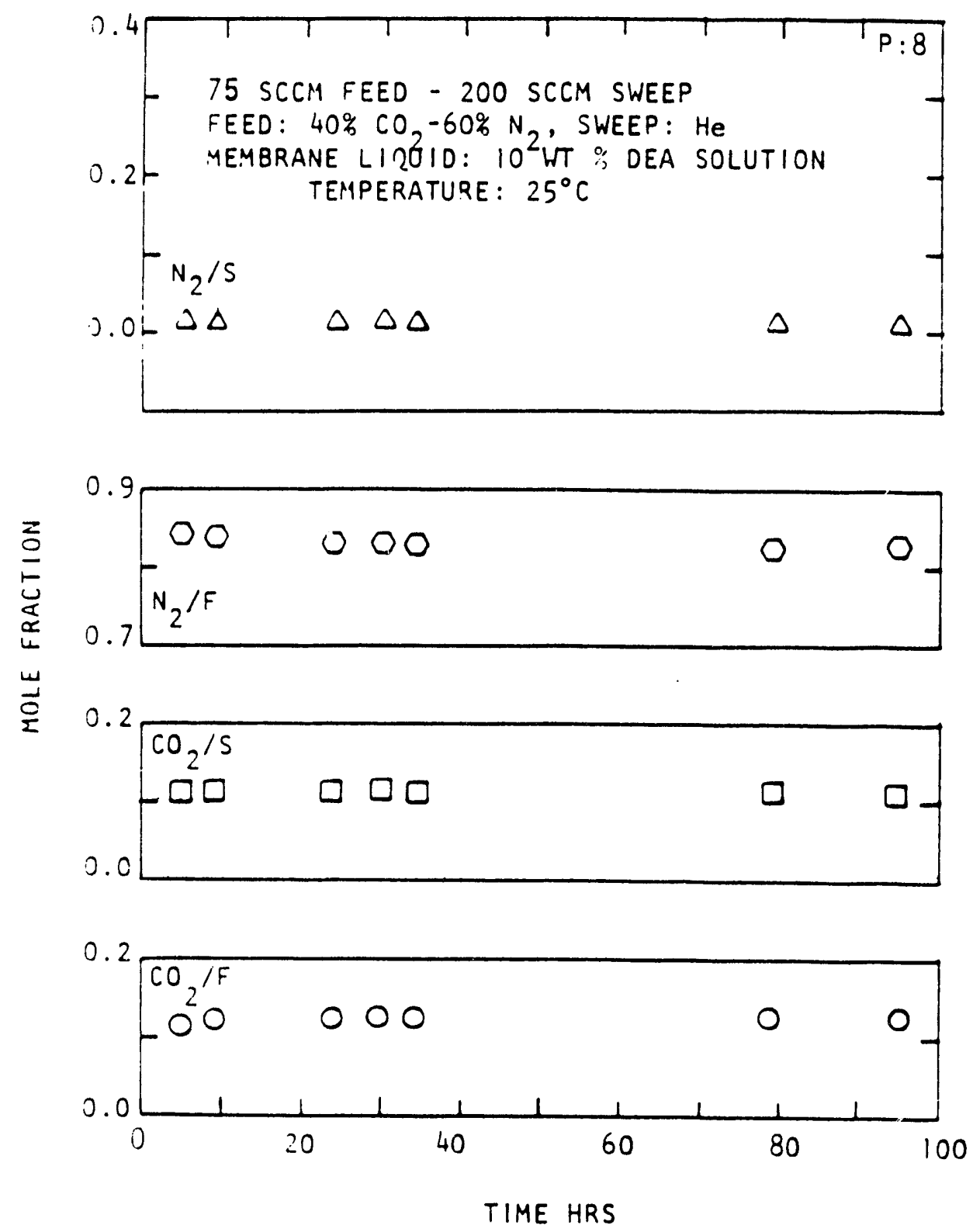

FIGURE $\quad 4-12$

CLM STABILITY STUDIES: SWEEP GAS MODE OF OPERATION WITH AQUEOUS 10 WT DEA SOLUTION AS MEMBRANE IN PERMEATOR \#8 

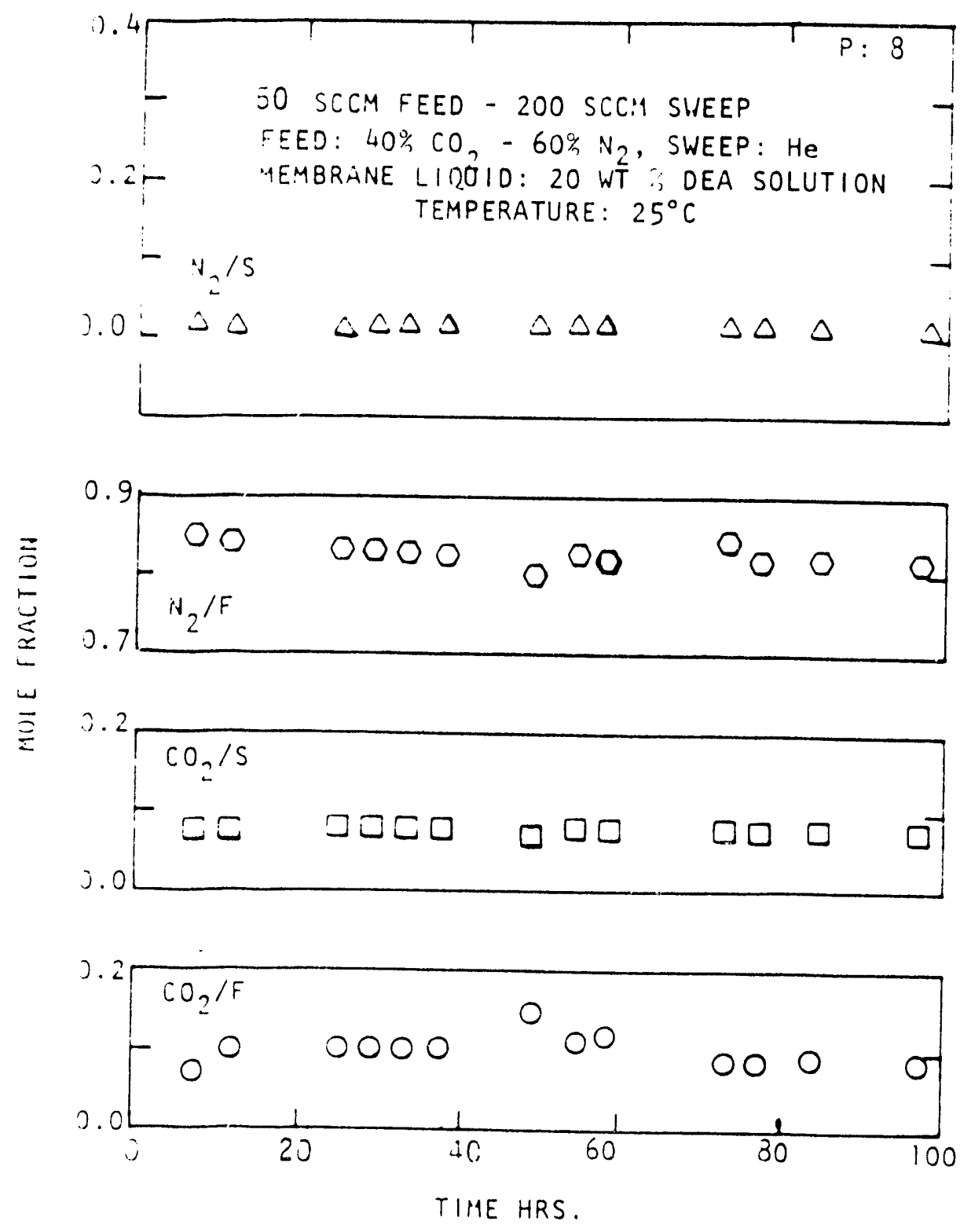

FIGURE $4-13$

CLM STABILITY STUDIES: SWEEP GAS MODE OF OPERATION WITH AQUEOUS 20 WT\% DEA SOLUTION AS MEMBRANE IN PERMEATOR \#8 $4-54$ 

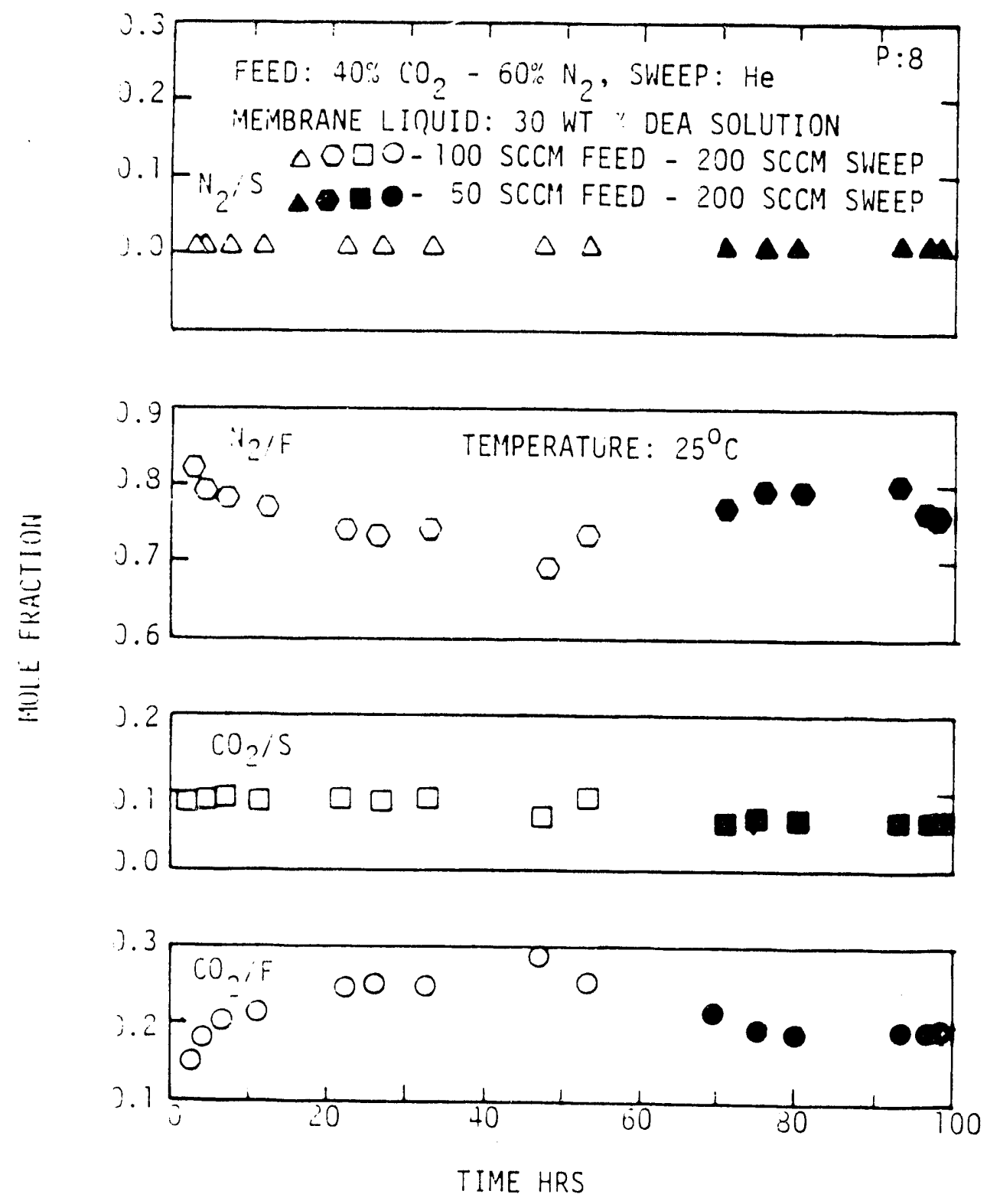

FIGURE 4-14

CLM STABILITY STUDIES: SWEEP GAS MODE OF OPERATION NITH AQUEOUS 30 WTO DEA SOLUTION AS MEMBRANE

IN PERMEATOR HO

4-55 
In order to carry out the separation study at an elevated temperature, a few modifications of the small-scale purification loop were made. Two membrane humidifiers were added to the existing feed and sweep gas humidifier vessels. These membrane humidifiers were kept in a separate constant temperature bath. The temperature of this bath was maintained around $\sim 60^{\circ} \mathrm{C}$ by a temperature regulator. The bath containing the permeator was maintained at $40^{\circ} \mathrm{C}$ by another temperature regulator. The connecting lines between the humidifiers and the permeator as well as the constant temperature baths were properly insulated to reduce the heat loss. Even with these modifications, however, precise control of the system temperature was difficult.

The purification performance is reported in Table 4-27. The system operation was stable. The $\mathrm{CO}_{2}$ composition in the purified gas stream varied from 3 to $7 \%$. Such a variation can be explained by the temperature fluctuations in the system over the long period. Comparing the data of Table 4-27 with that of low temperature $\left(25^{\circ} \mathrm{C}\right)$ run in Table 4-25, we see that a better quality of the purified gas stream was achieved. The gas permeation rate was certainly higher at higher temperature. However, the average $\mathrm{N}_{2}$ recovery was lower. As temperature increases, the $\mathrm{N}_{2}$ permeation rate also increases. Throughout the experiment, a slow increase in feed outlet flow rate was seen. This was probably due to a slow drying of the membrane liquid. To counteract this, membrane liquid recirculation with water addition from outside can be instituted. A better temperature and humidity control can be implemented for higher temperature runs.

Aqueous Solution of 20 w $\%$ DEA as Liquid Membrane and $40 \mathrm{x} \mathrm{CO}_{2}-60 \mathrm{CH}_{4}$ as Feed Gas

The simulated biogas mixture containing $40 \% \mathrm{CO}_{2}-60 \% \mathrm{CH}_{4}$ was introduced at a pressure of 45 psig. Pure $\mathrm{N}_{2}$ gas was used as sweep gas. Both gas streams were humidified. The flow rates of feed and sweep gas were $50 \mathrm{sccm}$ and $200 \mathrm{sccm}$, respectively. Pure helium was used to pressurize the membrane liquid storage tank. Permeator \#8 was used.

The experimental results of 12 hours' purification are shown in Figure 4-15. A steady performance was obtained in 6 hours. Simulation results and the steady 


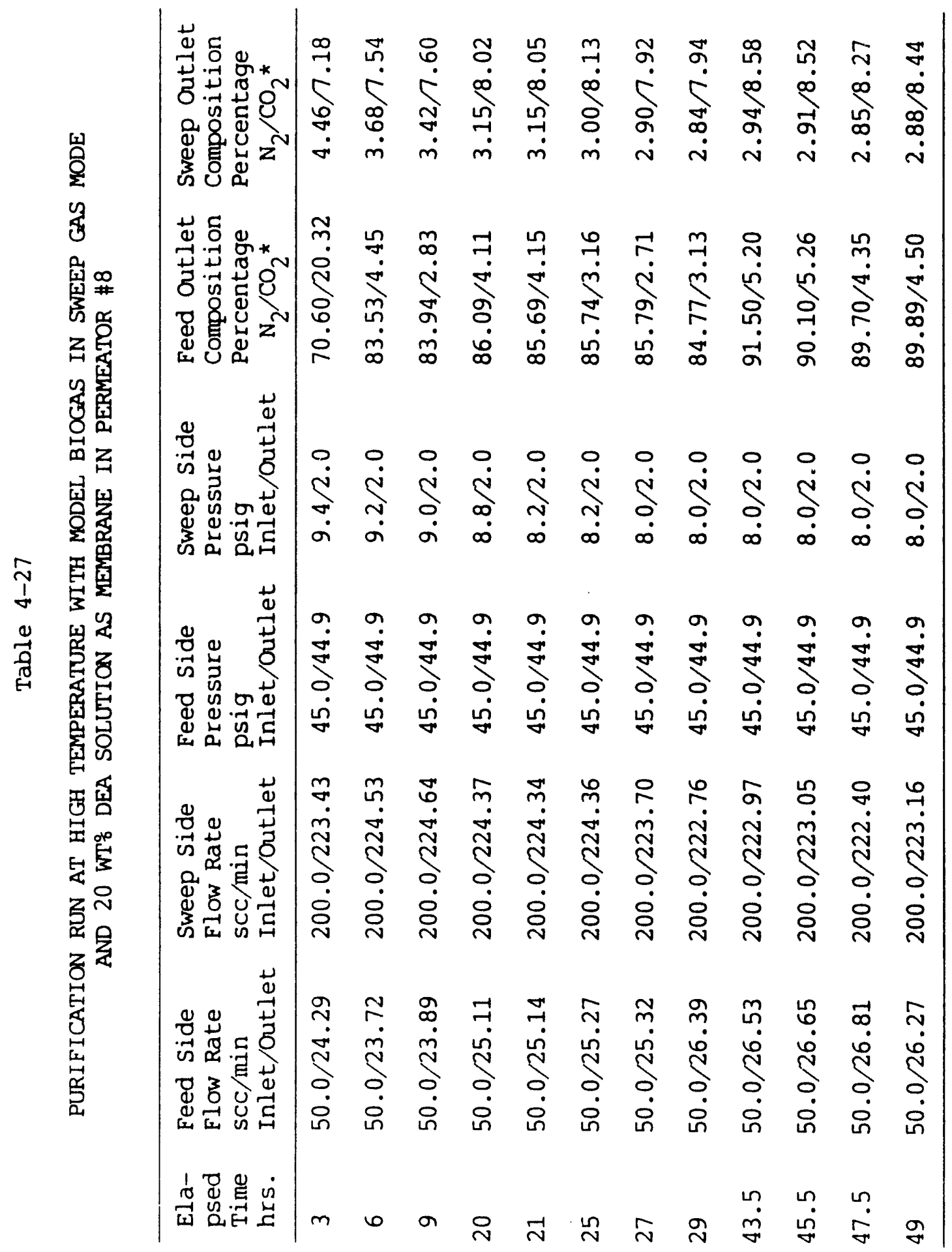




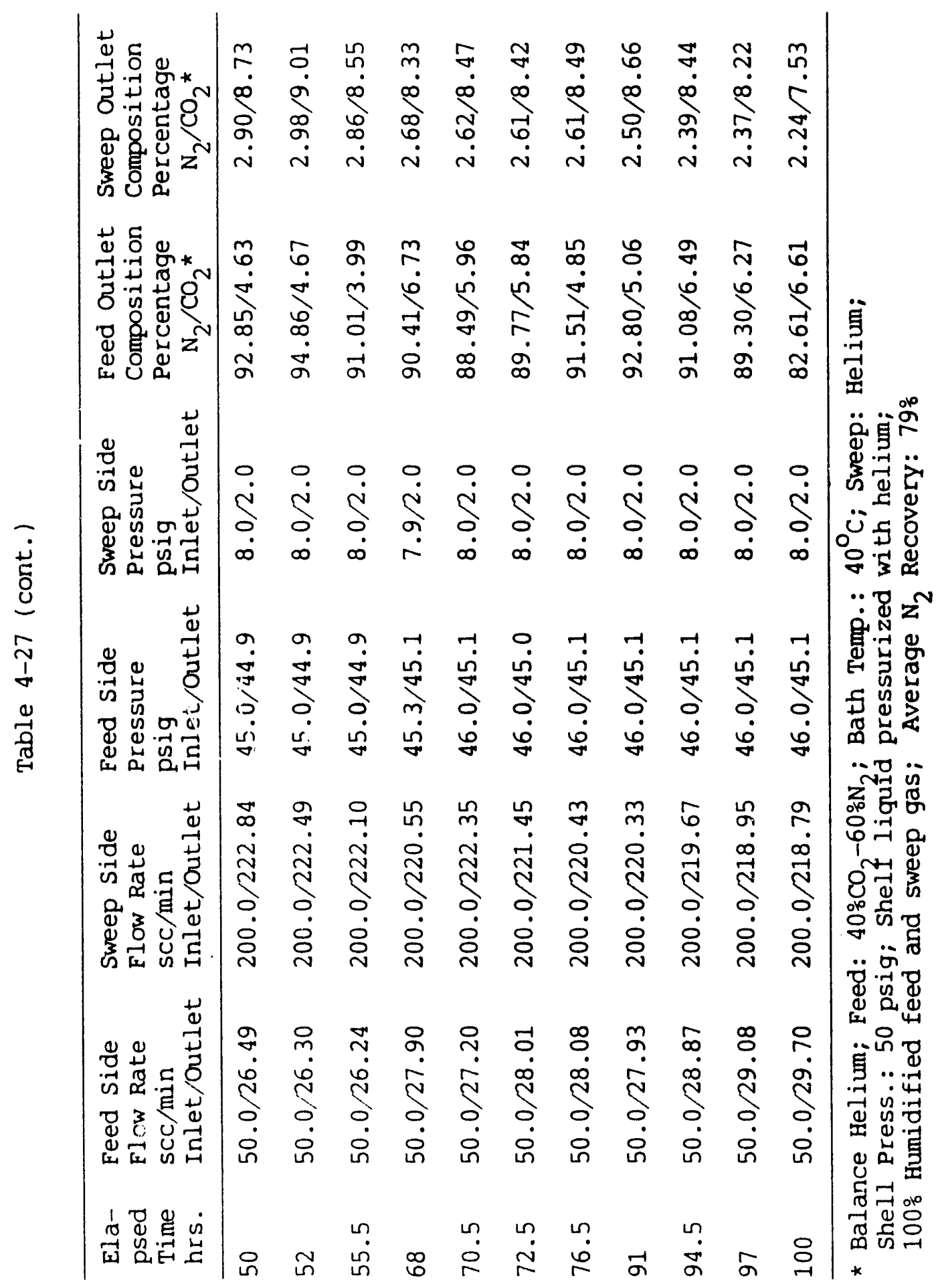



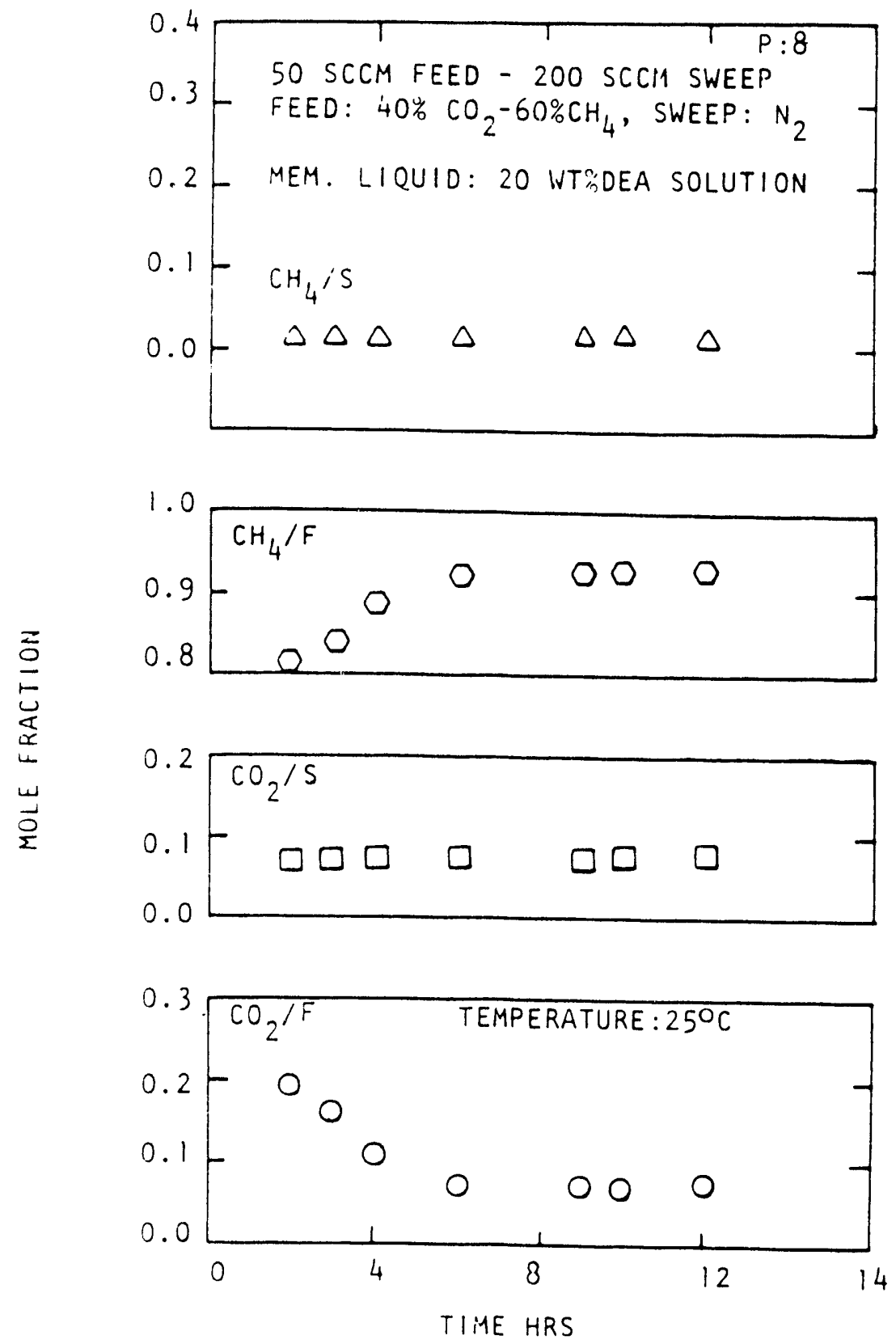

FIGURE 4-15

PERFORMANCE OF MOUEL BIOGAS PURIFICATION WITH AQUEOUS 20 WT: 
state data are also compared in Table 4-28. The average $\mathrm{CH}_{4}$ recovery was around $93 \%$. The purified feed gas stream contained only $7 \% \mathrm{CO}_{2}$. Note that, the effective permeation area in permeator $\# 8$ is around $30 \%$ of that of a 5 -ft-long permeator (e.g., permeator \#3 or \#5). The permeation rate of sweep $\mathrm{N}_{2}$ into feed stream was very low. The average $\mathrm{N}_{2}$ concentration is around $2 \%$.

These experiments demonstrate that both high purification and recovery can be simultaneously achieved in a small permeator when $20 \mathrm{wt} \%$ DEA in water is used as the liquid membrane. This is very encouraging because, unlike $30 \mathrm{wt} \% \mathrm{~K}_{2} \mathrm{CO}_{3}$ solution liquid membrane, the increased selectivity has been achieved without sacrificing the permeability to any extent.

\section{Aqueous Solution of 10 wt\% DRA as Liquid Membrane and} $5 \% \mathrm{CO}_{2}-95 \% \mathrm{~N}_{2}$ as Feed Gas

Effect of partial pressure on $\mathrm{CO}_{2}$ permeability was studied in HFCLM permeator $\# 8$ by using feed gas containing a lower percentage of $\mathrm{CO}_{2}$. Experiments were carried out with humidified feed and sweep gas streams. Feed gas was maintained at 45 psig in all the runs. Steady state data with 10 wt\% DEA as liquid membrane are shown in Table 4-29. The data suggest much higher permeability of $\mathrm{CO}_{2}$ compared to high pressure data (Table 4-21).

After completion of the separation run with DEA as liquid membrane, another experiment with pure water as liquid membrane has been carried using permeator \#8. At first the permeator was cleaned with water and then dried with nitrogen gas. This was done in order to determine the effect, if any, of long-time DEA exposure of the permeator. Dry feed and sweep gas flow rates of $50 \mathrm{sccm}$ and 200 sccm were used in this particular run. The data presented in Table 4-30 compare very well with the data taken earlier under identical conditions (4th row; Table 4-16). This implies that long-term exposure to the DEA solutions did not affect the microporous polypropylene hollow fibers at all.

Aqueous Solution of NMP as Liquid Membrane and $40 \% \mathrm{CO}_{2}-60 \% \mathrm{~N}_{2}$ as Feed Gas

Permeator \#9 had been used for gas purification studies with 1-methyl2-pyrrolidinone (NMP) solution as contained liquid membrane. First a solution 


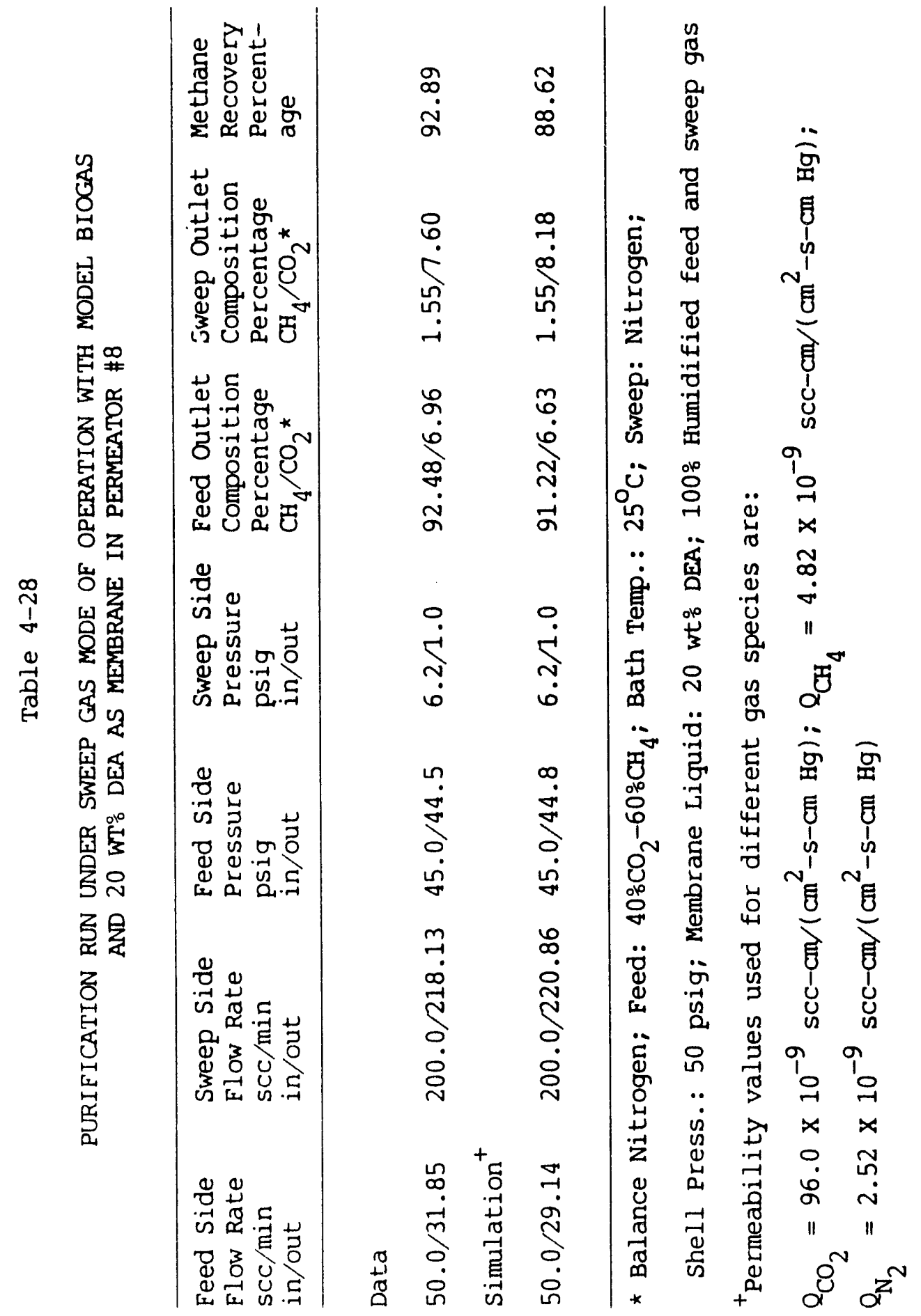




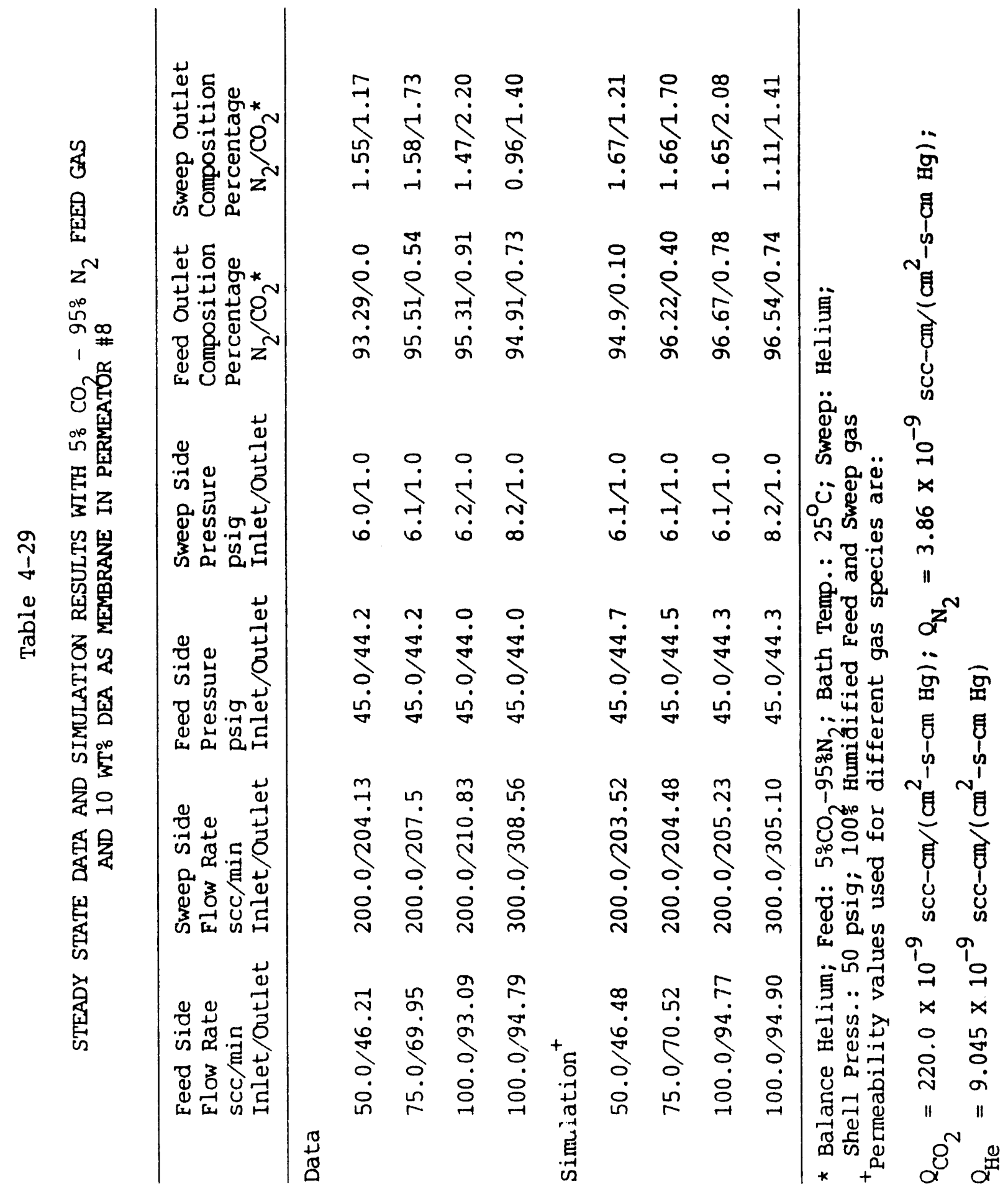




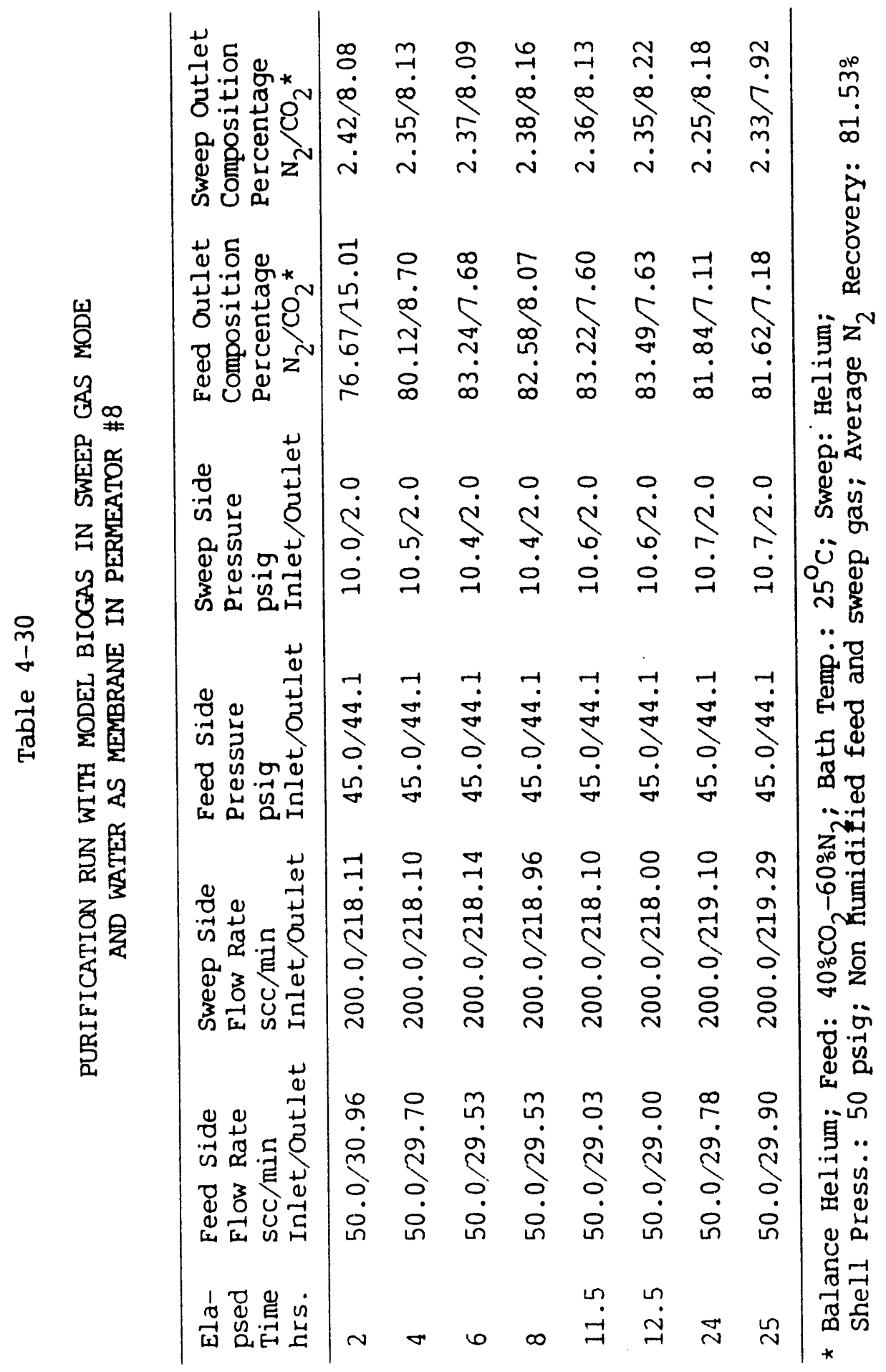


containing 80 vol\% NMP and 20 vol\% water was tried as a liquid membrane. As Table 4-31 indicates, the level of purification was low (about $23 \% \mathrm{CO}_{2}$ in the purified gas stream). A slow and steady increase in sweep inlet pressure led us to believe that the membrane liquid was penetrating the pores of the microporous sweep fibers due to high pressure differential ( $\Delta P$ ) between the liquid membrane and the sweep gas stream. Therefore, after 5 hours, the sweep outlet pressure was raised from 2 psig to a level of 10 psig in order to reduce this pressure differential. The feed flow rate was also lowered. The reduction in feed flow rate resulted in a slightly better performance. However, the sweep gas pressure continued to increase slowly and after a period of time membrane liquid was clearly visible in the connecting lines of the sweep side and the experiment was discontinued.

After thorough washing and drying, the permeator was again used for gas purification studies. This time a liquid mixture having a lower NMP concentration was selected as a membrane liquid. The contained liquid membrane was made of 70 vol\% NMP and 30 vol\% water. The experiment was carried out at a lower pressure to begin with. A steady and stable performance was observed under these conditions, as shown in Table 4-32. The permeation rate of $\mathrm{N}_{2}$ into the sweep stream was very low. The nitrogen recovery was about $92 \%$. The $\mathrm{CO}_{2}$ composition in the feed outlet was, however, relatively high (27\%).

After 25 hours of experimental run, the feed as well as membrane liquid storage tank pressures were increased, keeping the feed and sweep flow rates the same. The run was continued for another 50 hours. A stable performance was again observed. The experimental results are reported in Table 4-33. The overall performance improved slightly due to a higher pressure of the feed stream. The $\mathrm{CO}_{2}$ composition in the feed outlet dropped to about $24 \%$. The nitrogen loss in the sweep stream was also very low.

These experiments suggest that stable performance is achievable with a membrane liquid having a composition of 70 vol\% NMP and 30 vol\% water. Very high nitrogen recovery was obtained when NMP solution was used as a liquid membrane, but the level of purification was low compared to the other membrane liquids we have studied. 


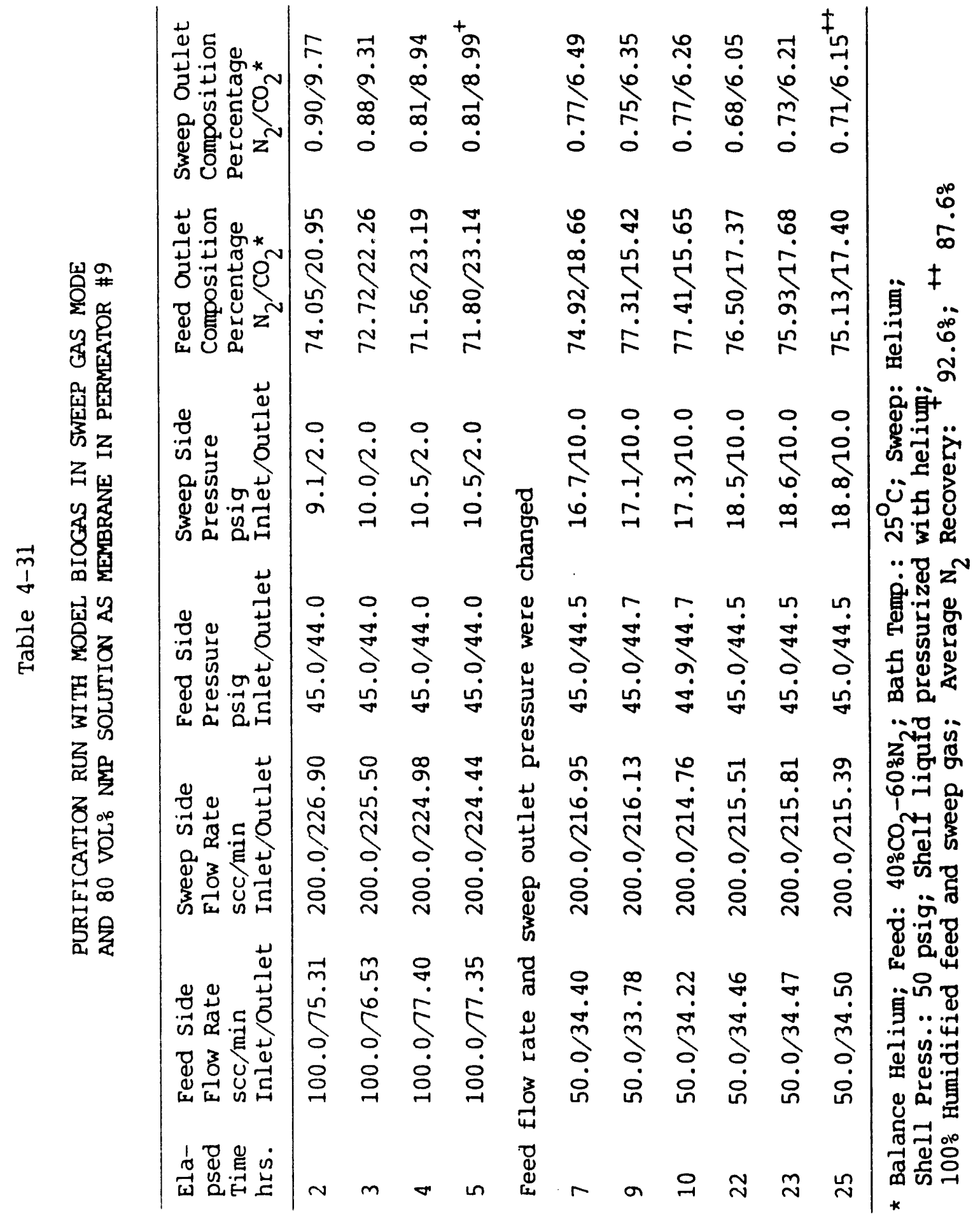




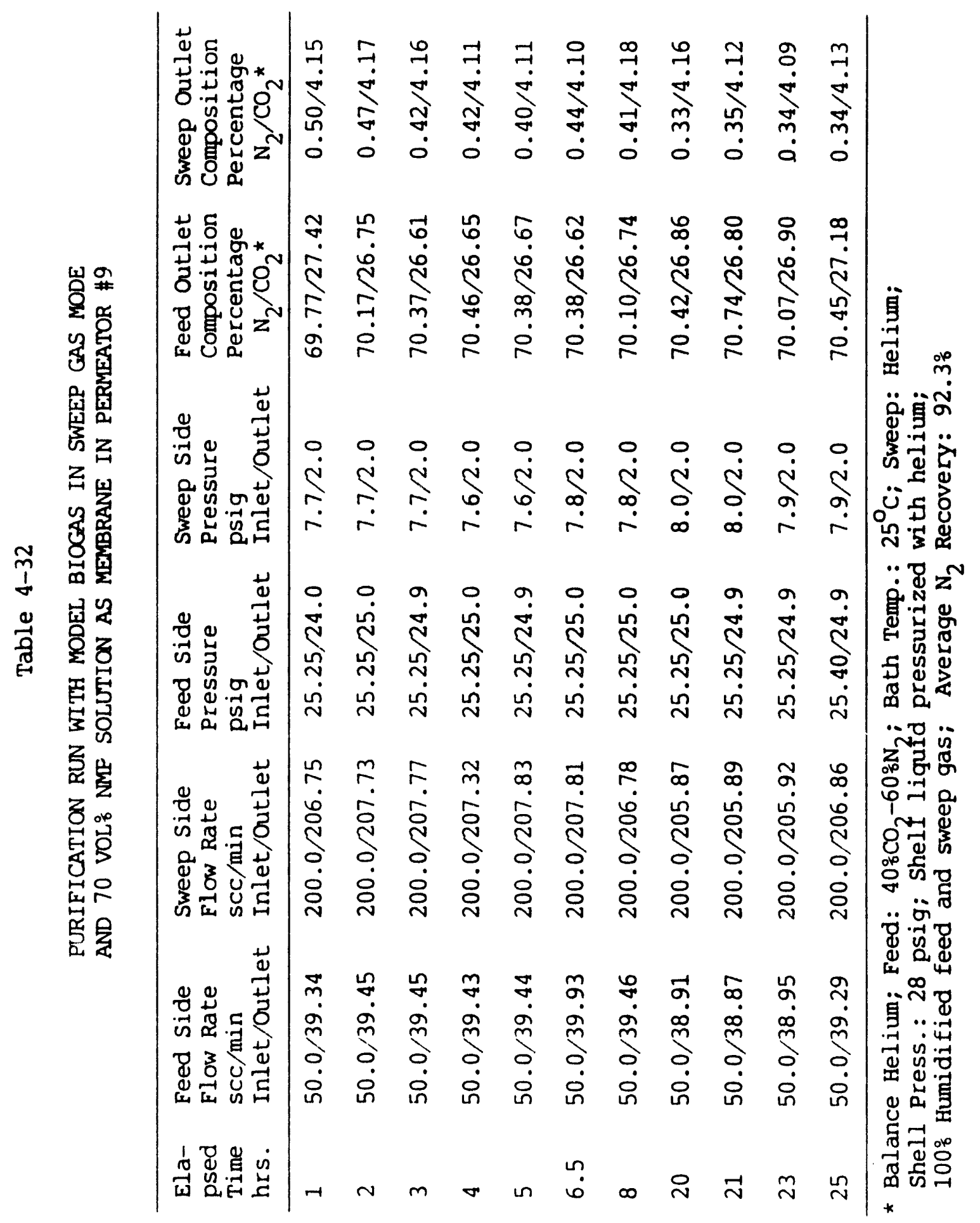




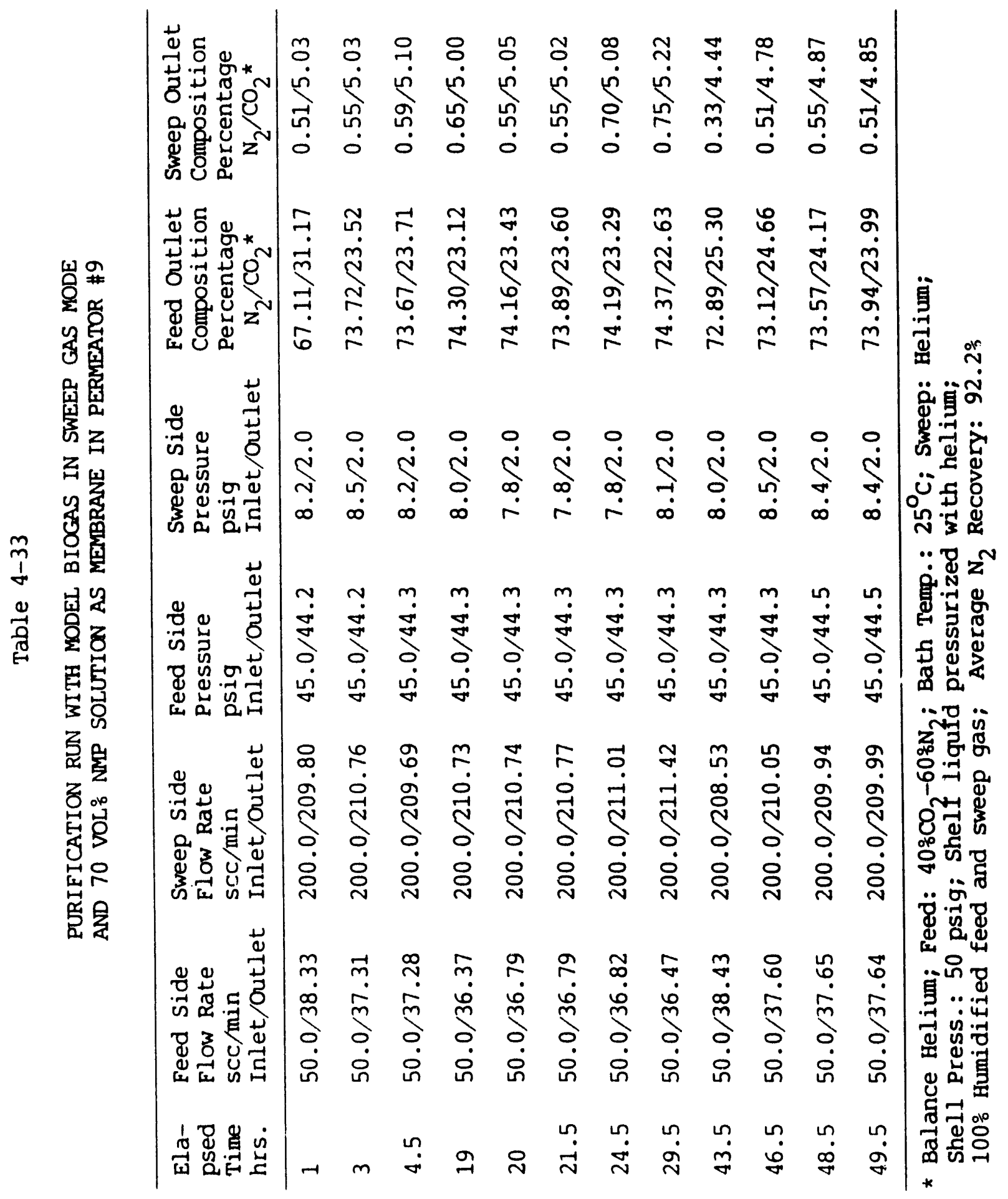


PURIFICATION STUDIES OF MODEL BIOGAS MIXTURES IN CONVENIIONAL POLTMRRIC MEMBRANE MODE WITH DIFFERENT LIQUID MEMBRANES

Model biogas purification in a HFCLM permeator, operated in the conventional polymeric membrane mode, was carried out as follows. Model feed biogas mixture consisting of $40 \% \mathrm{CO}_{2}-60 \% \mathrm{~N}_{2}$ was introduced through one set of fibers at an inlet pressure of $45 \mathrm{psig}$. No sweep gas or vacuum was applied through the other set. The necessary permeate pressure was built up by the permeated gas itself. The particular permeate end, which was earlier used for sweep gas introduction, was kept closed to maintain a countercurrent flow of the permeate stream.

Water as Liquid Membrane and $40 \% \mathrm{CO}_{2}-60 \% \mathrm{~N}_{2}$ as Feed Gas

Results obtained at five different flow rates in permeator \#3 are reported in Table 4-34. Note that, at the lowest feed flow rate of $50 \mathrm{sccm}$, the purified feed gas stream contained only about $5 \% \mathrm{CO}_{2}$. However, the nitrogen recovery in this case was around $70 \%$. The data and the similation results are plotted in Figure 4-16. The simulation results are in very good agreement with the experimental data.

The same data are presented in a different way in Figure 4-17, where a plot of stage cut (ratio of the permeate flow rate to the feed flow rate) against mole fractions of $\mathrm{N}_{2}$ and $\mathrm{CO}_{2}$ in feed outlet stream (commonly known as reject stream) is show?. As usual an increase in stage cut increases $\mathrm{N}_{2}$ in the reject stream and improves the quality of the purified gas.

Similar type of experiments were carried out using permeator \#6. Here, the permeate gas was withdrawn through the larger bore fibers. Steady state results are reported in Table 4-35. Even though a very high percentage of nitrogen was recovered in these experiments, the extent of feed purification was low. For example, at the lowest feed flow rate of $50 \mathrm{sccm}$, the purified gas stream contains about $20 \% \mathrm{CO}_{2}$. This performance is reasonably good when we consider that permeator \#6 is only 2 feet long and the membrane area in permeator \#6 is only $40 \%$ that of permeator \#3. The data and the numerical simulation results are shown in Figure 4-18. The model predicts the performance quite well. 


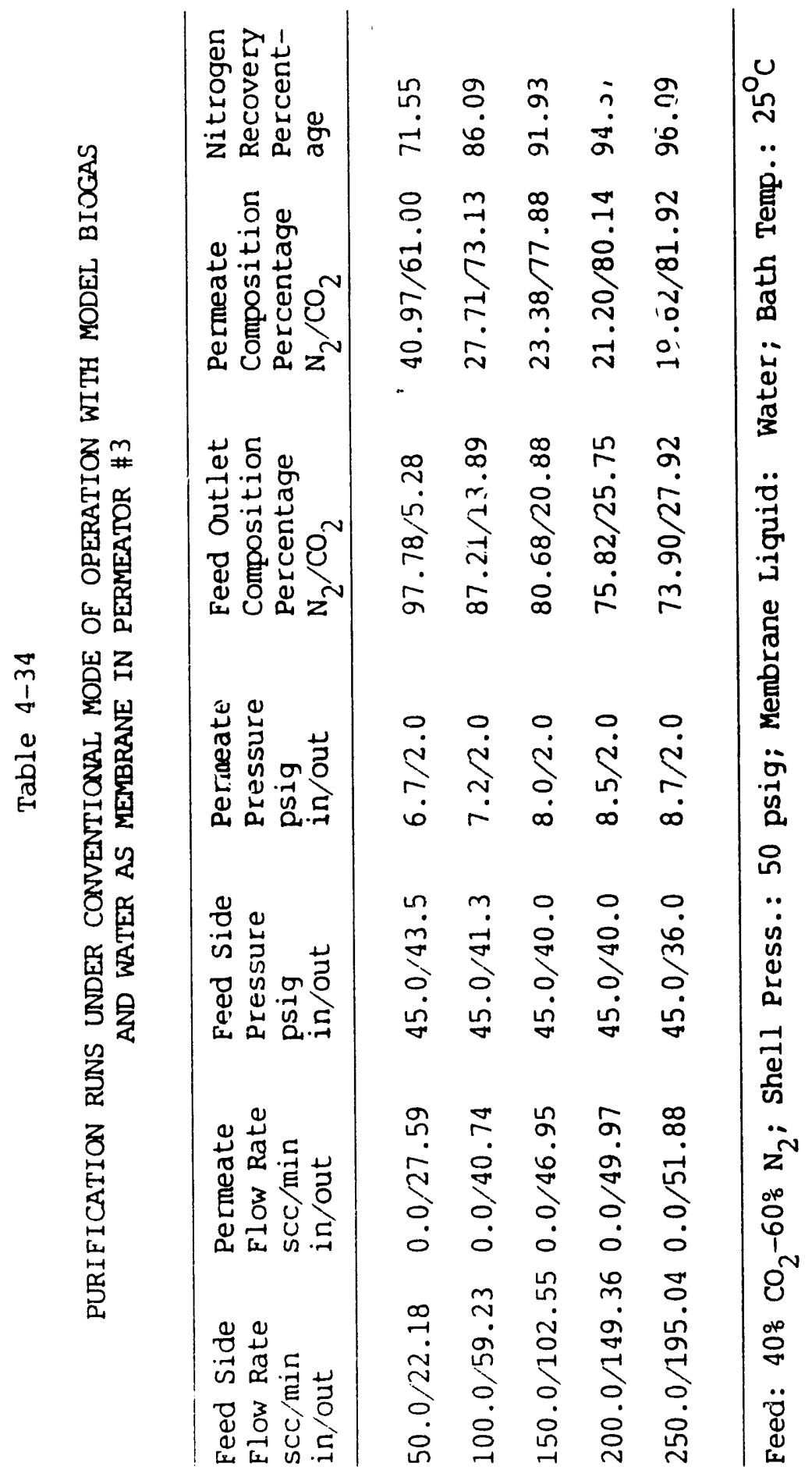




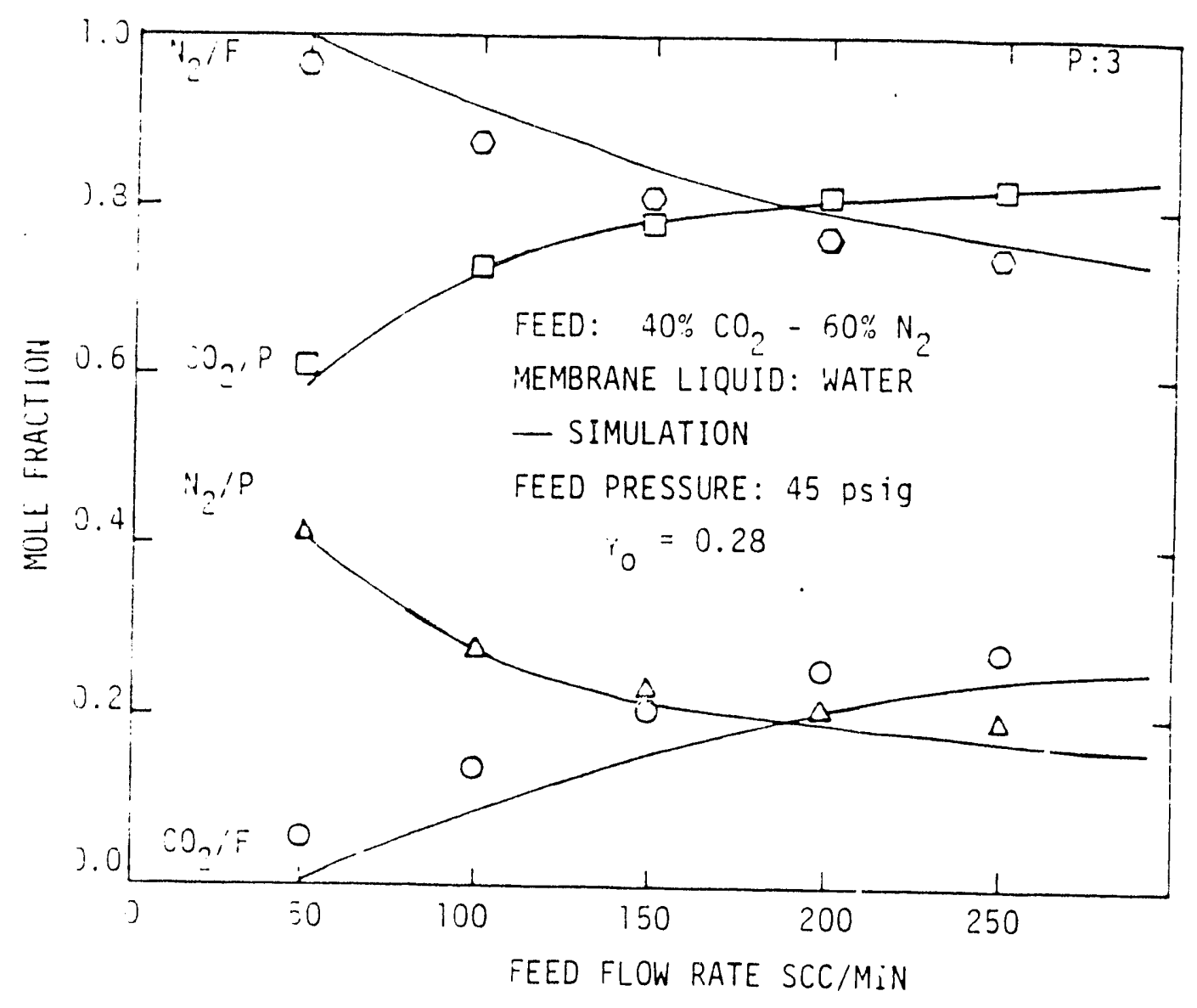

FIGURE $4-16$

FEED FLOW VARIATION STUDIES: CONVENTIONAL POLYMERIC MEMBRANE MODE OF OPERATION IN PERMEATOR \# 3 


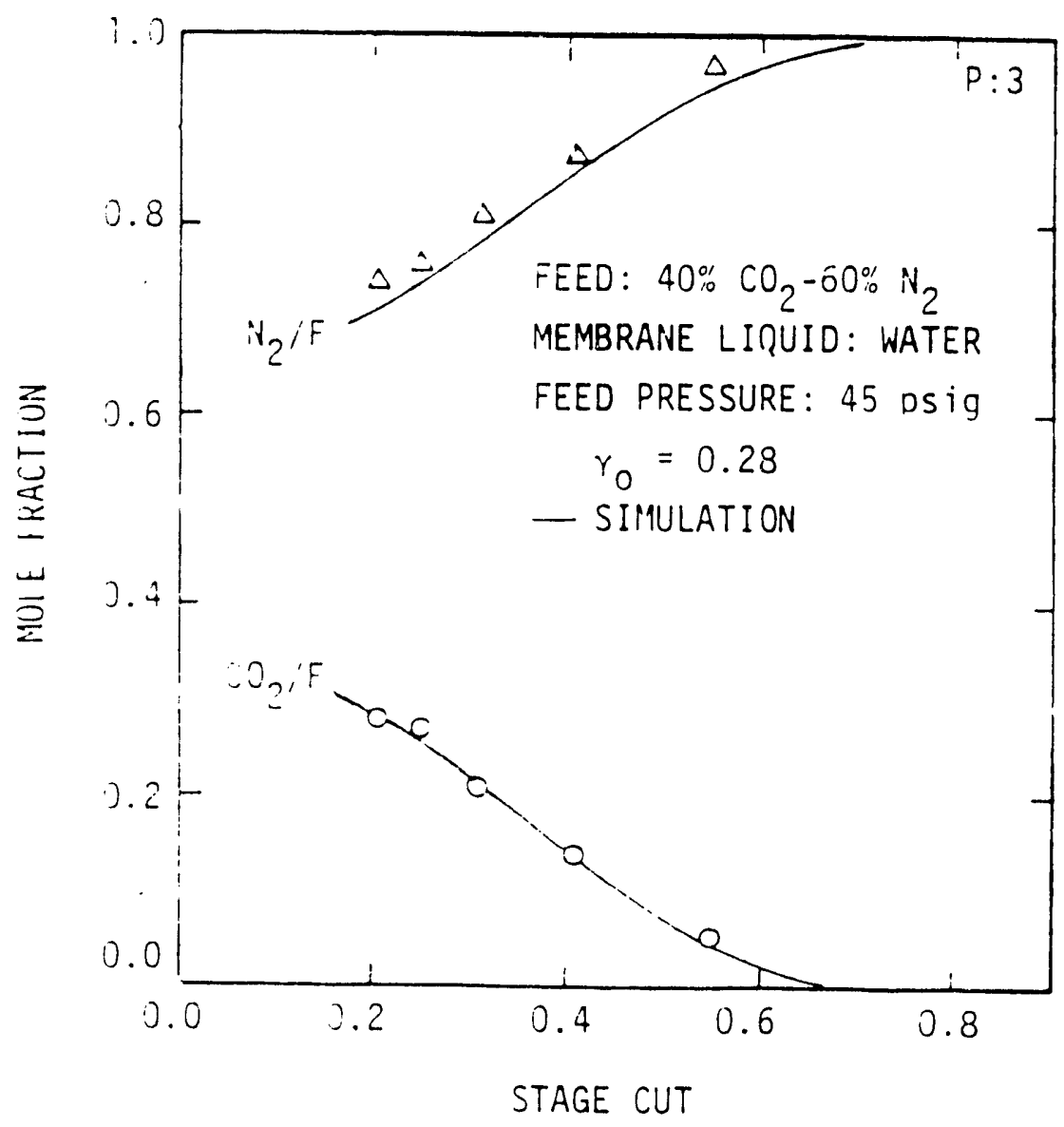

FIGURE $4-17$

SEPARATION UNDER CONVENTIONAL POLYMERIC MEMBRANE MODE: STAGE CUT VS. COMPOSITION IN PERMEATOR \# 3 


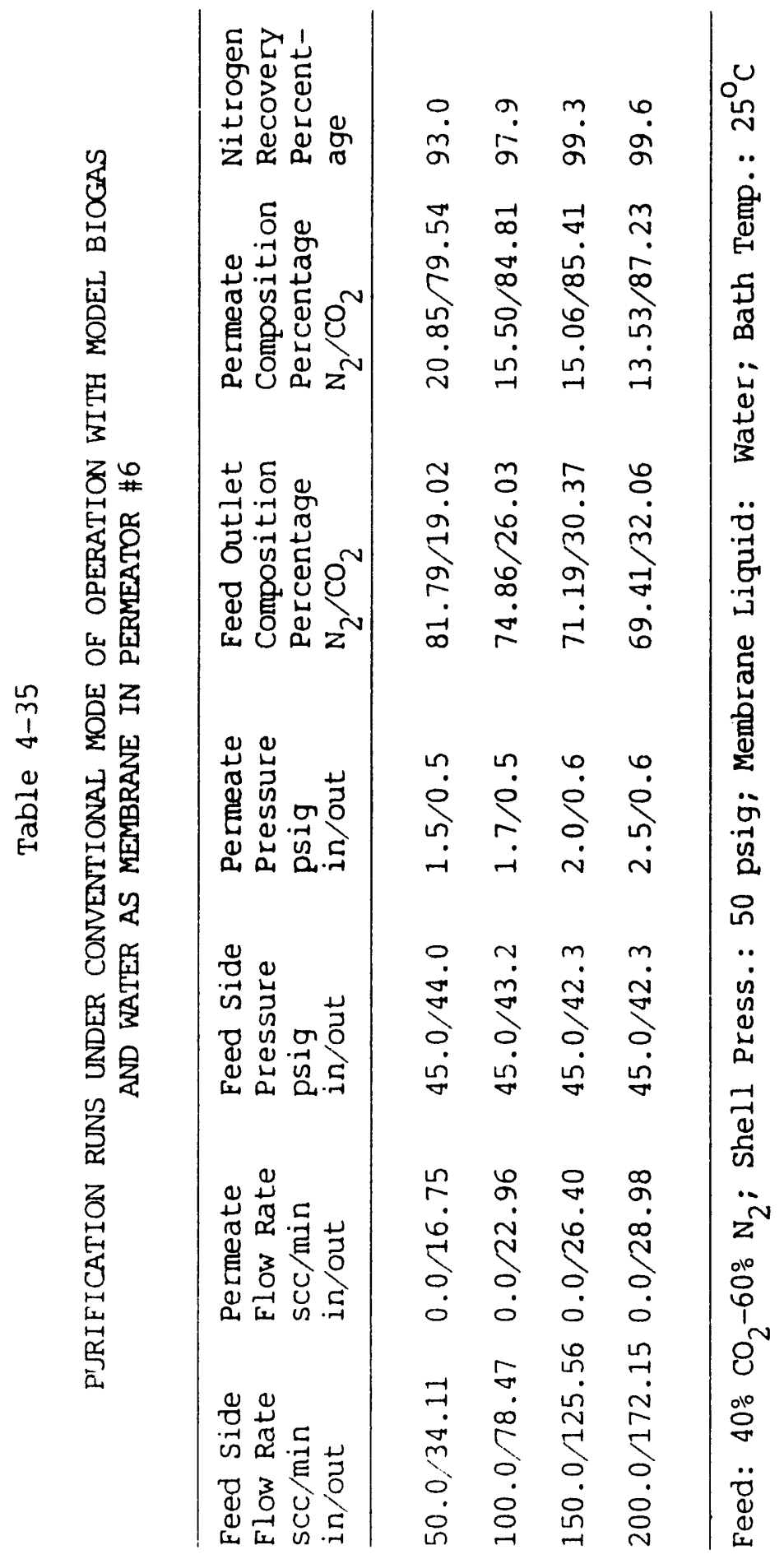




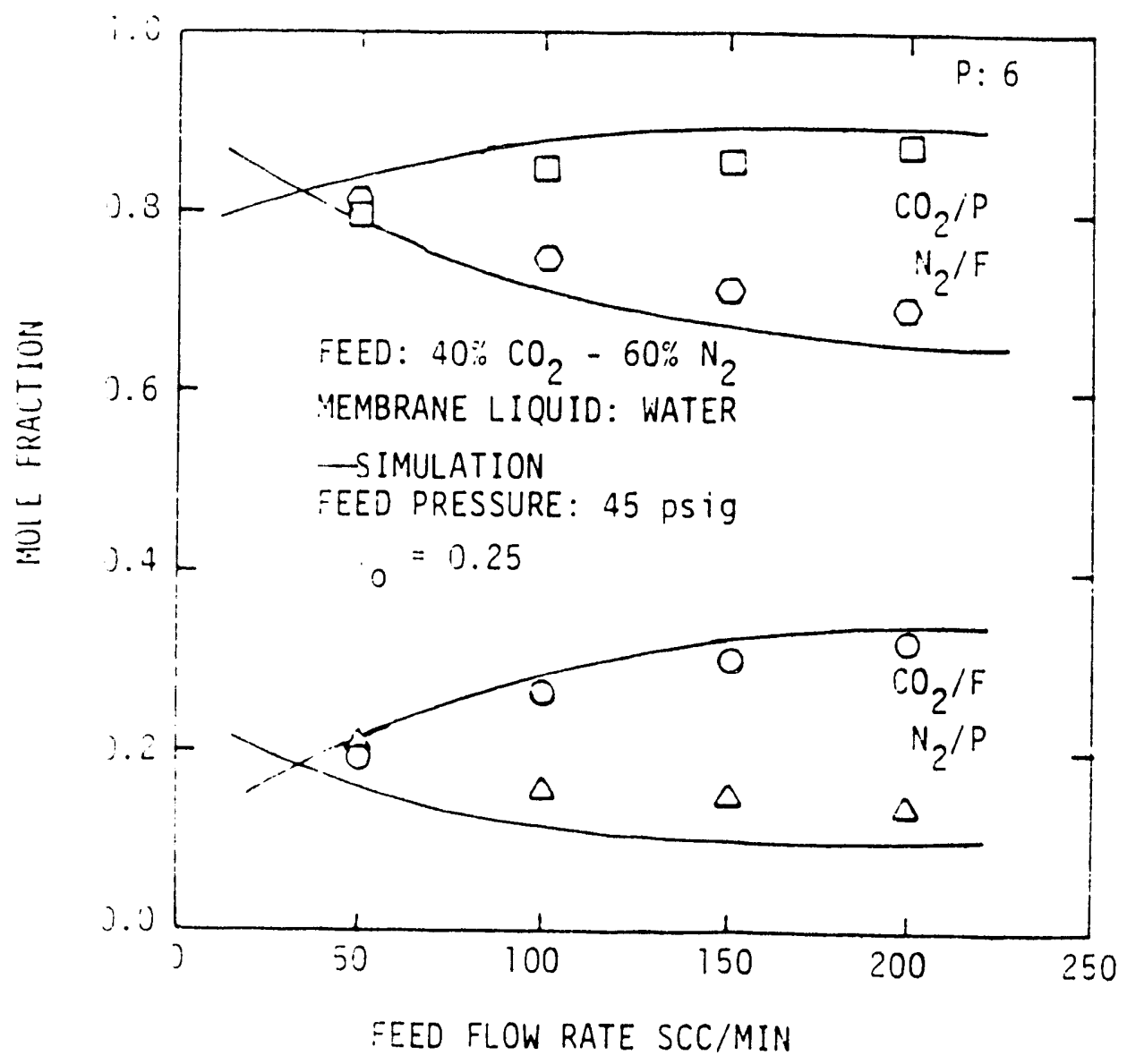

FIGURE $4-18$

FEED FLOW VARIATION STUDIES: CONVENTIONAL POLYMERIC MEMBRANE MODE OF OPERATION IN PERMEATOR \# 6 
The data for the permeator \#8 is shown in Table 4-36. The purified gas stream at $50 \mathrm{sccm}$ flow rate contains about $20 \% \mathrm{CO}_{2}$. Note that, the effective length of permeator \#8 is around $30 \%$ that of permeator \#3. Experimental data and numerical simulation results are shown in Figure 4-19. Although, permeator \#8 has smaller membrane area than permeator \#6, a similar quality in the purified gas was obtained due to a lower effective liquid membrane thickness in permeator $\# 8$. The deviation in the permeate composition $\left(\mathrm{CO}_{2} / \mathrm{P}\right.$ and $\left.\mathrm{N}_{2} / \mathrm{P}\right)$ was due to leakage of air into the permeate stream which was diluted with respect to $\mathrm{CO}_{2}$ content. Since it occurred beyond the flow meter it did not affect the permeation process which is evident from the very good prediction of the performance of the feed side compared to permeate side.

Separation runs with permeator \#3 were also carried out in a fashion somewhat different from the conventional mode of operation; both ends of the permeate fibers were kept open. The permeate flow was cocurrent-countercurrent in nature. Pure water was used as membrane liquid. This mode was identified as the split permeation mode (Majumdar, 1982). The steady state results for five different flow rates are shown in Table 4-37. Figure 4-20 shows the corresponding plot. Comparing the data with those obtained with conventional mode of operation (Table 4-34), it is clear that the overall permeation rate is considerably higher in split permeation mode of operation. The higher permeation rate is a result of an increase in driving force across the liquid membrane, which arises due to a reduction in permeate fiber lumen pressure drop. However, the quality of the purified stream is not necessarily better in this case. At lower feed flow rates, the nitrogen content in the purified gas stream is slightly lower in the present mode of operation and the same is marginally higher at high flow rates. At higher flow rates, the effect of a reduction in the permeate fiber lumen pressure drop resulted in an improved performance.

30 wt $\% \mathrm{~K}_{2} \mathrm{CO}_{3}$ Solution as Liquid Membrane and $40 \% \mathrm{CO}_{2}-60 \% \mathrm{~N}_{2}$ as Feed Gas

Separation of model biogas mixture was also carried out under conventional mode of operation with $30 \mathrm{wt} \% \mathrm{~K}_{2} \mathrm{CO}_{3}$ solution as liquid membrane in permeator \#5. The experiments were conducted at four different flow rates e.g., 50, 75, 100 and $150 \mathrm{sccm}$. The steady state data are presented in Table 4-38. Feed inlet 


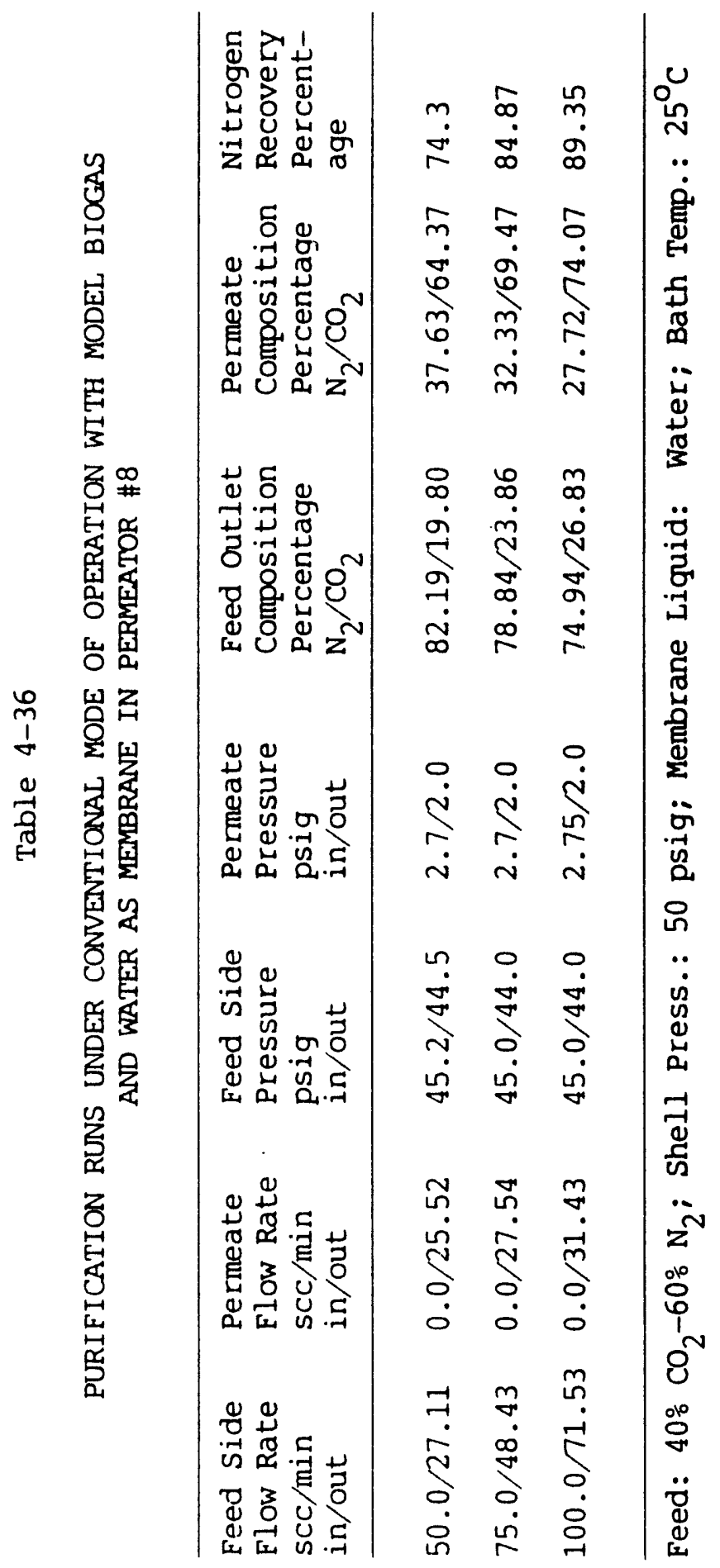




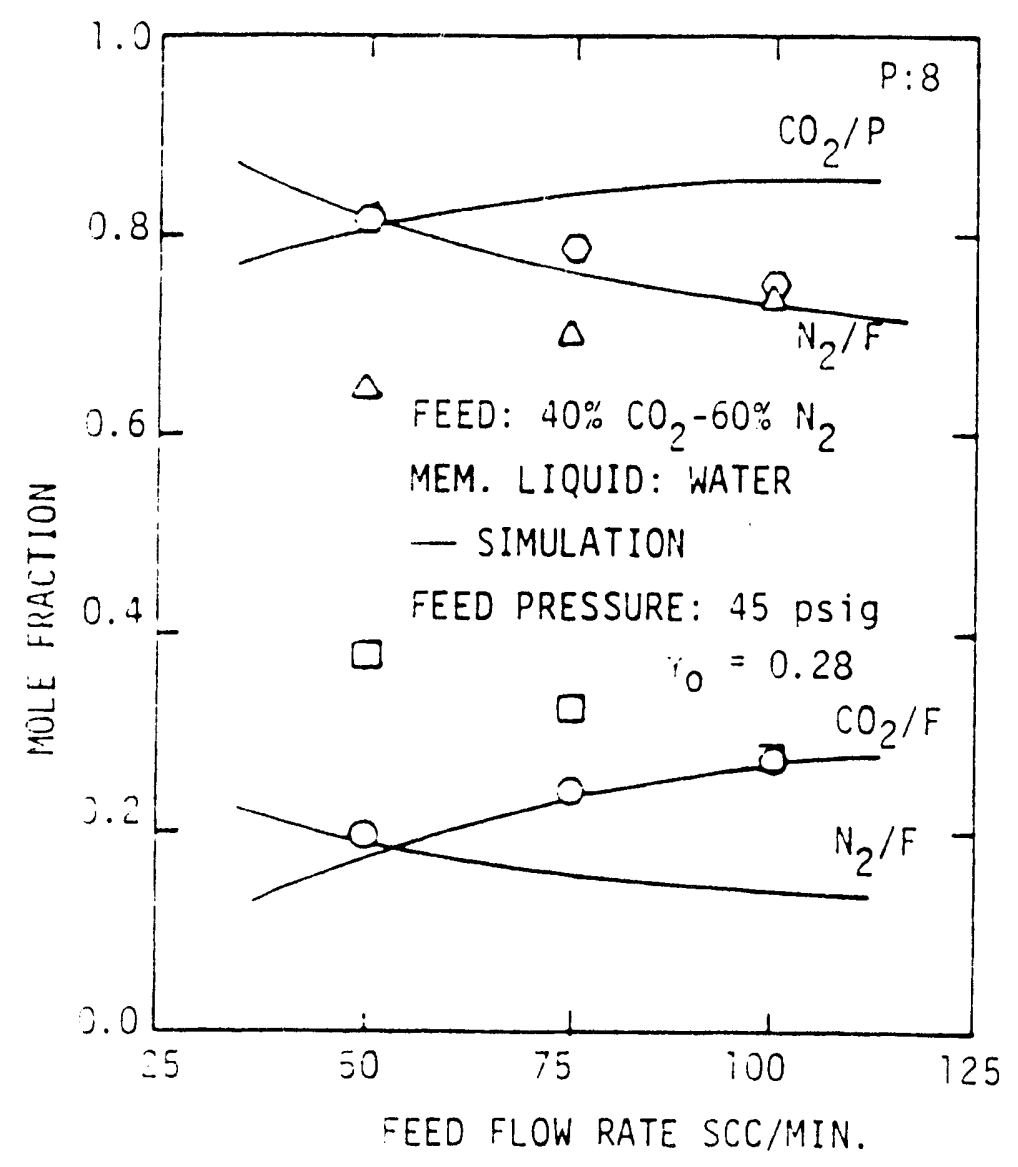

FIGURE $4-19$

EEED FLOW VARIATION STUDIES: CONVENTIONAL POLYMERIC IIEMBRANE MODE OF OPERATION IN PERMEATOR \#8 


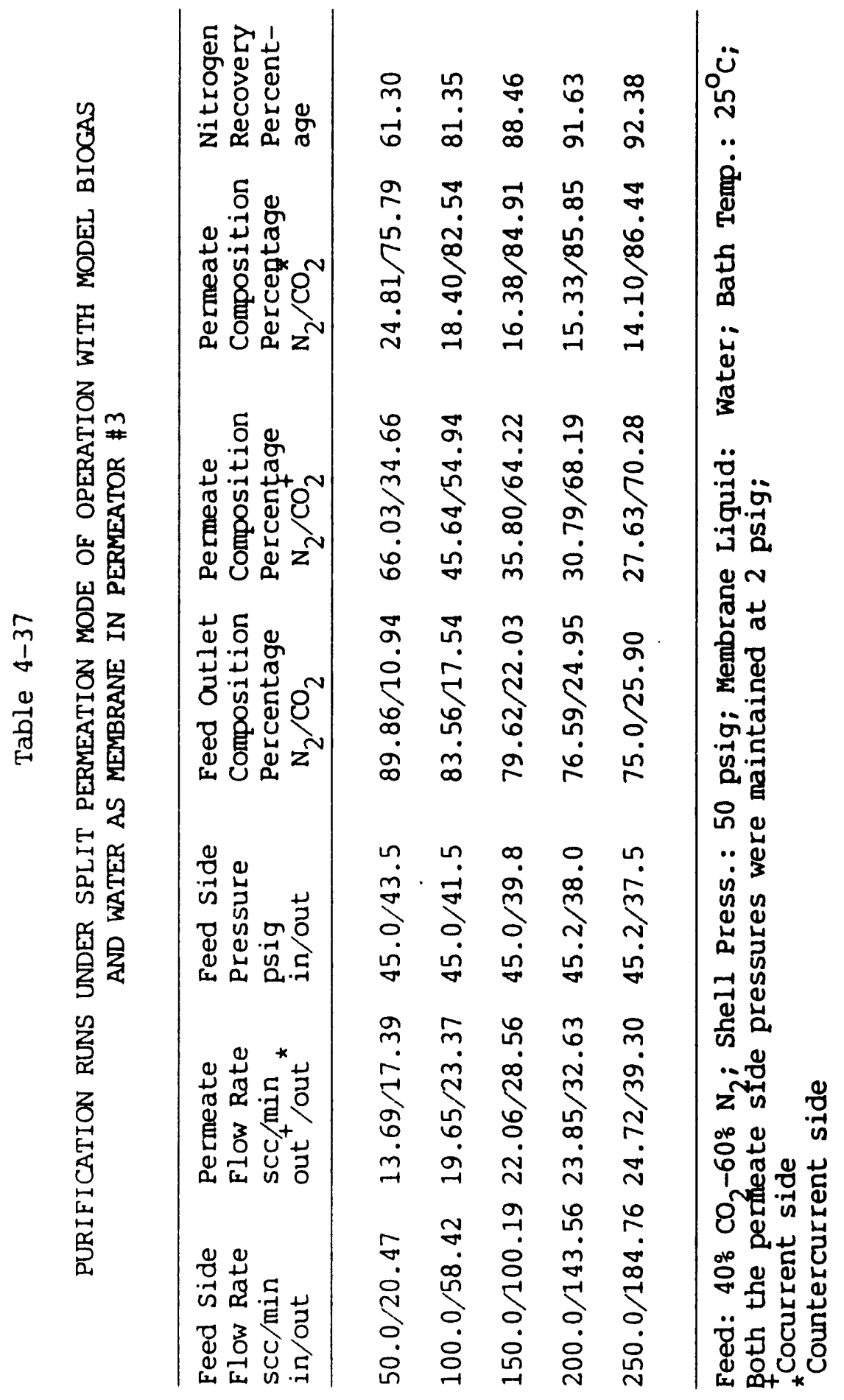




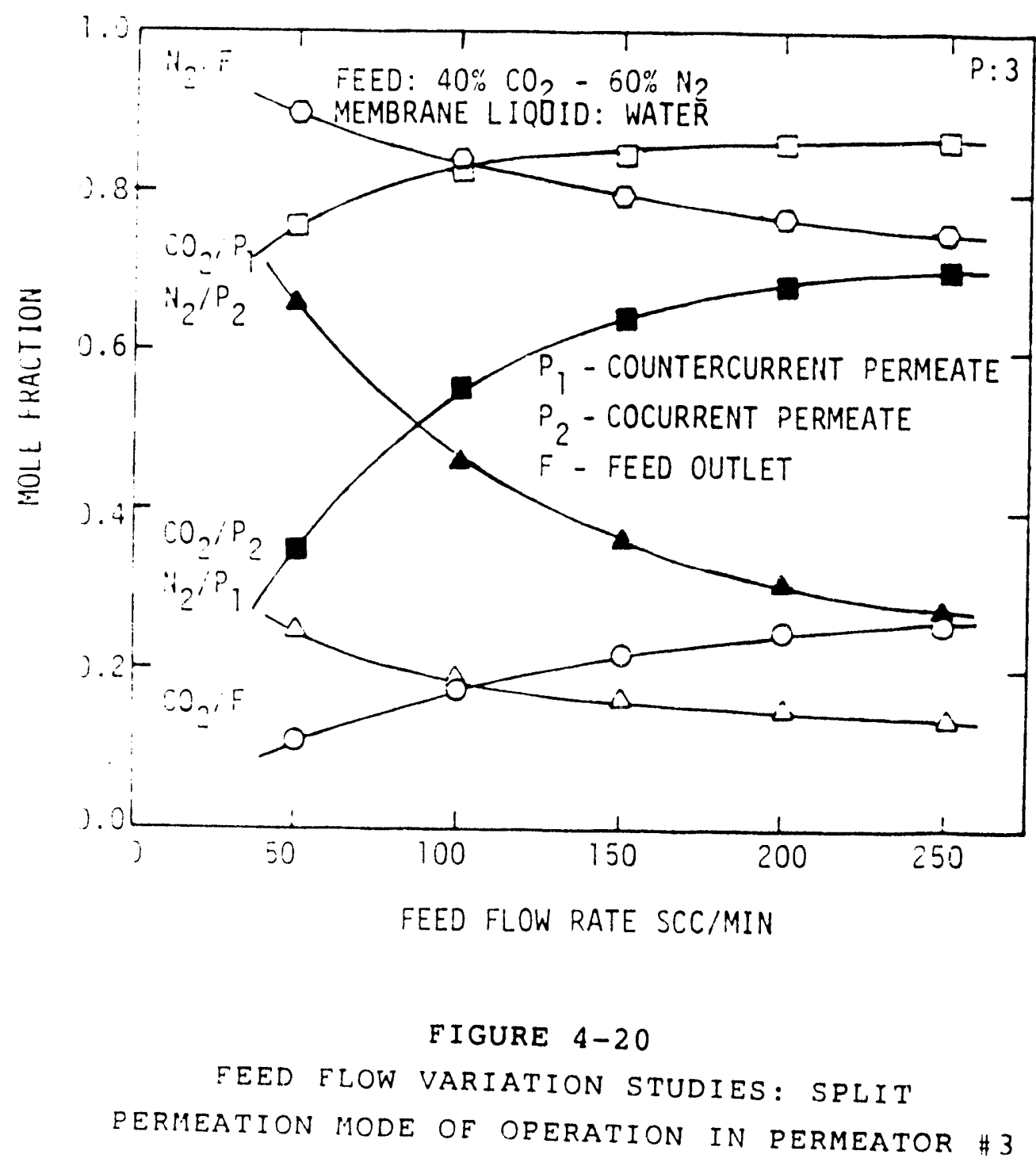




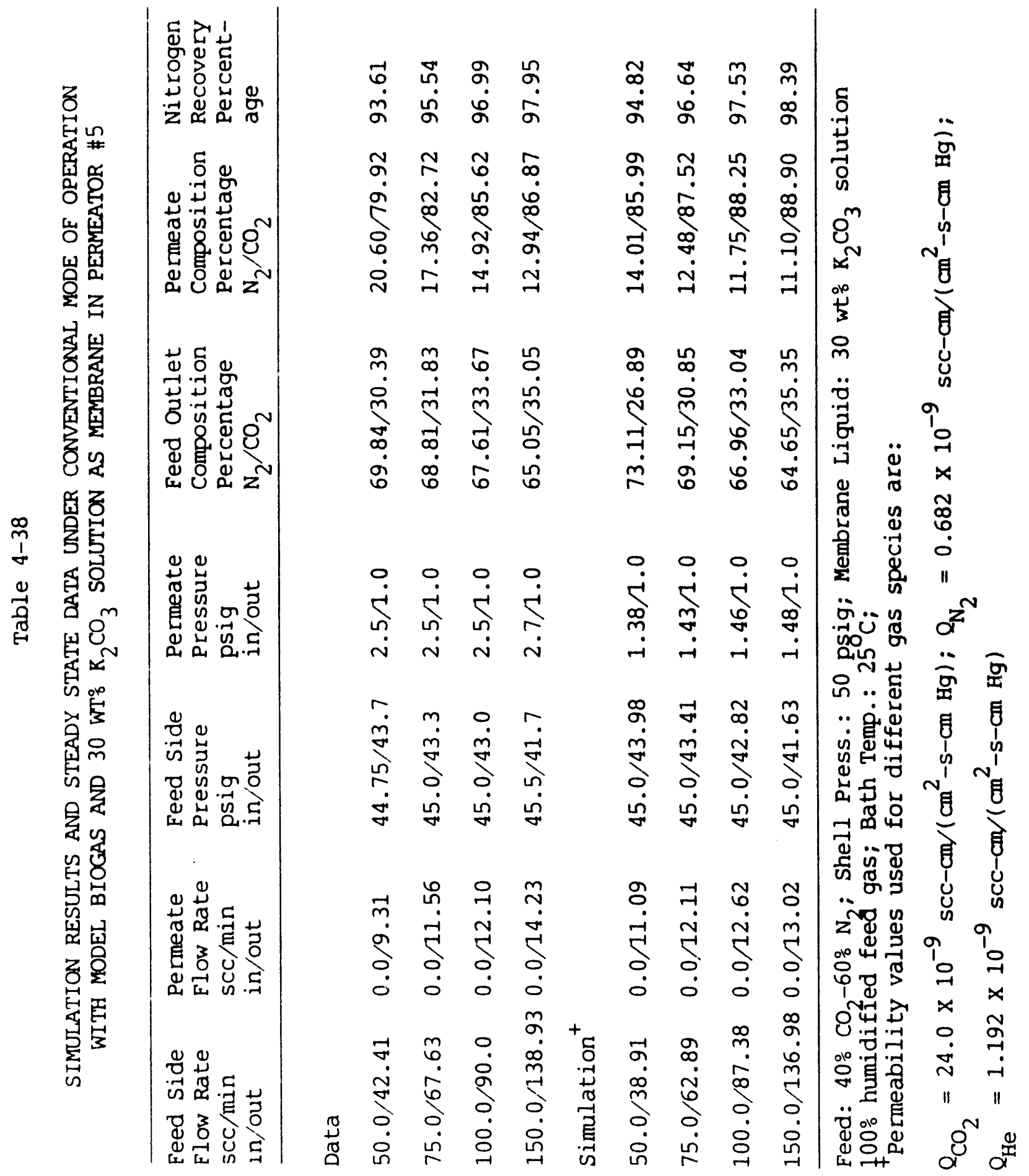


pressure of 45 psig was mainlained in all the cases. Nitrogen recovery was very high, but the extent of feed gas pulfication was quite low in such mode. The $\mathrm{CO}_{2}$ composition in the feed oullet was very high and it increased with an increase in feed flow rate. This is due to the significantly lower permeability values of the gases through che salt solution at $25^{\circ} \mathrm{C}$. The steady state data and numerical results for the same conditions are compared in Table 4-38 and graphically demonstrated in Figure 4-21. A plot of stage cut vs composition is shown in Figure 4-22. Note that the simulation was based on permeability of $\mathrm{CO}_{2}$ with no facilitation. This is expected as the partial pressure driving force is drastically reduced in this mode and the carrier in the membrane liquid gets saturated. Moreover, there may be slow fouling of the microporous hollow fibers by microcrystals of salt.

\section{PURIPICATION STUDIES OF MODEL BIOGAS MIXTURES UNDER VACUUM MODE OF OPERATION}

Model biogas purification studies under vacuum mode of operation were carried out mainly with pure water as a liquid membrane. Either $40 \% \mathrm{CO}_{2}-60 \% \mathrm{~N}_{2}$ or $40 \%$ $\mathrm{CO}_{2}-60 \% \mathrm{CH}_{4}$ was used as a feed gas. In all experiments with water membrane, the feed gas stream was introduced without any humidification. Two separation experiments were carried out with $7 \mathrm{wt} \% \mathrm{~K}_{2} \mathrm{CO}_{3}$ solution as a contained liquid membrane.

Water as Liquid Membrane and $40 \% \mathrm{CO}_{2}-60 \% \mathrm{~N}_{2}$ as Feed Gas

Experimental data were taken at three different feed pressures using permeator \#3. The steady state data at feed pressures 30,35 and 40 psig are reported in Table 4-39. Three different feed gas flow rates (e.g., 100, 150 and $200 \mathrm{sccm}$ ) were used in each case. The above experimental data as well as results from numerical simulation of the model are plotted in Figures 4-23, 4-24 and 4-25, respectively.

As expected, the $\mathrm{CO}_{2}$ content in the purified gas increased with an increase in feed flow rate. At a low feed flow rate of $100 \mathrm{sccm}$, the $\mathrm{CO}_{2}$ percentage in the purified gas was found to be $3-4 \%$. A comparison of the data in these three tables reveals that, at any fixed ralue of tlow rate, the stage cut (ratio of the permeate flow rate to the feed flow rate) increased with an increase in 


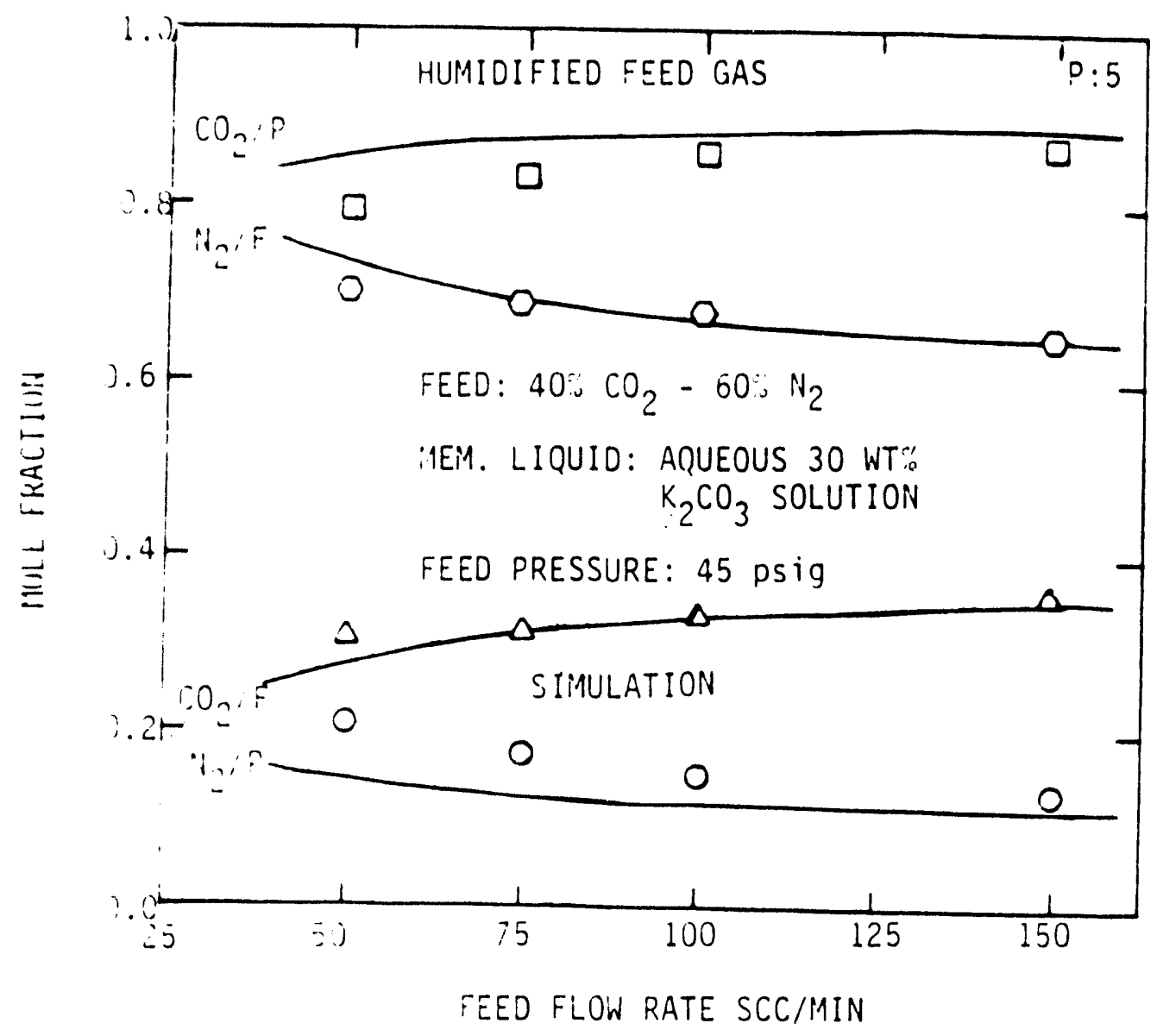

FIGURE $4-21$

FEED FLOW VARIATION STUDIES: CONVENTIONAL POLYMERIC MEMBRANE MODE OF OPERATION IN PERMEATOR \# 5 


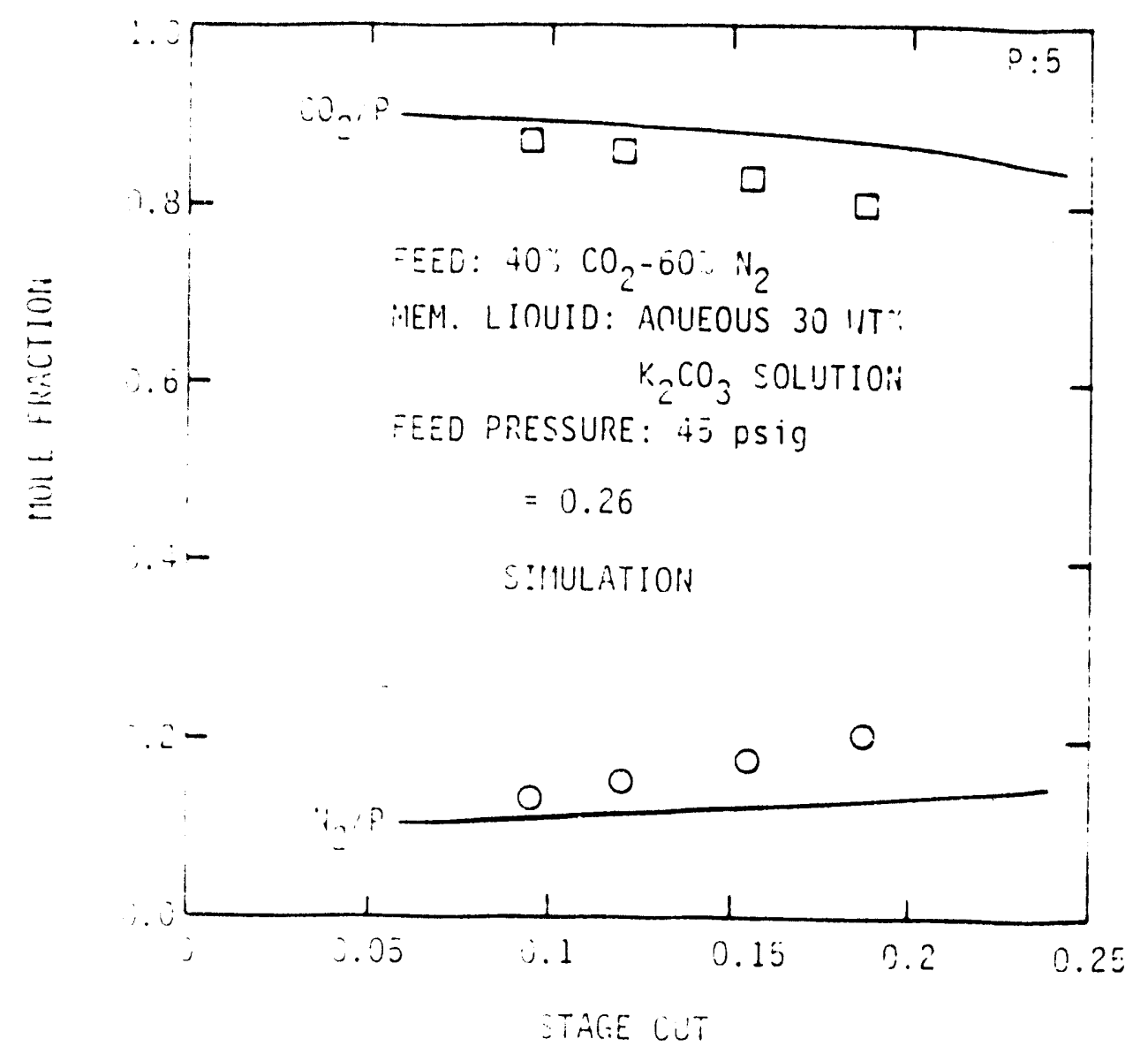

FIGURE 4-22

SEPARATION UNDER CONVENTIONAL POLYMERIC

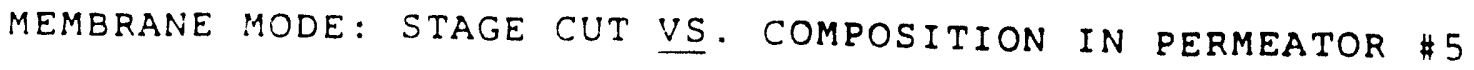




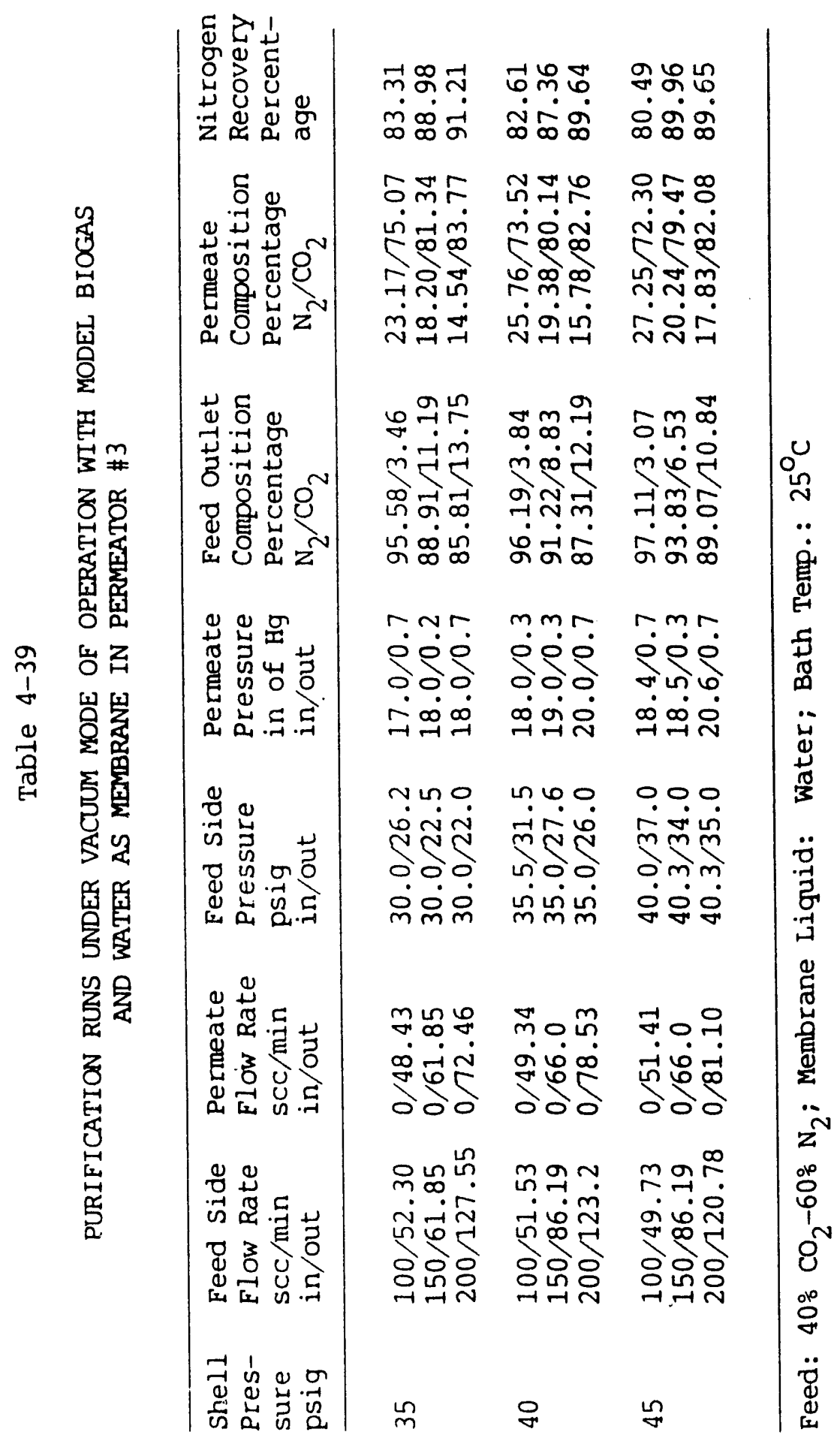



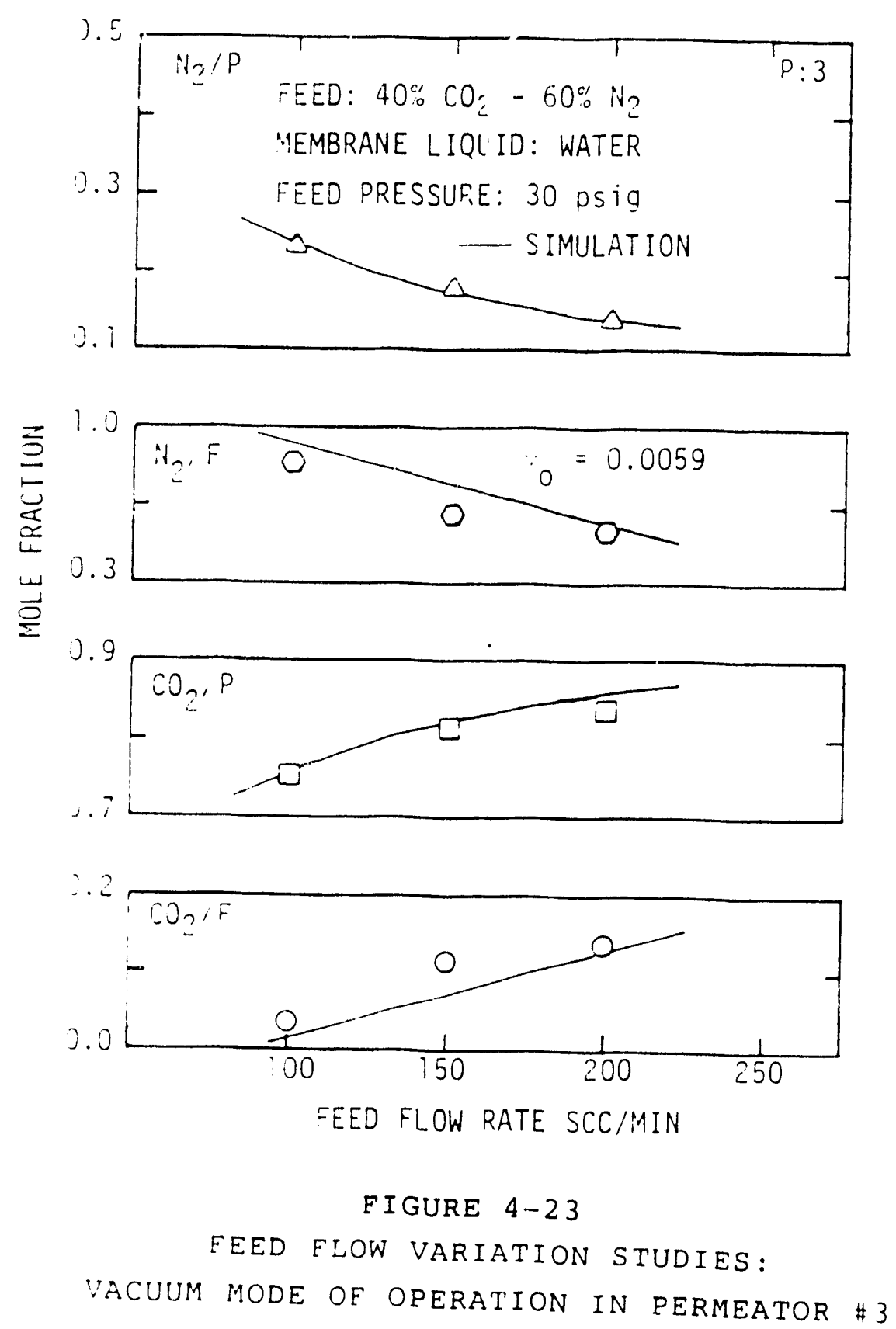


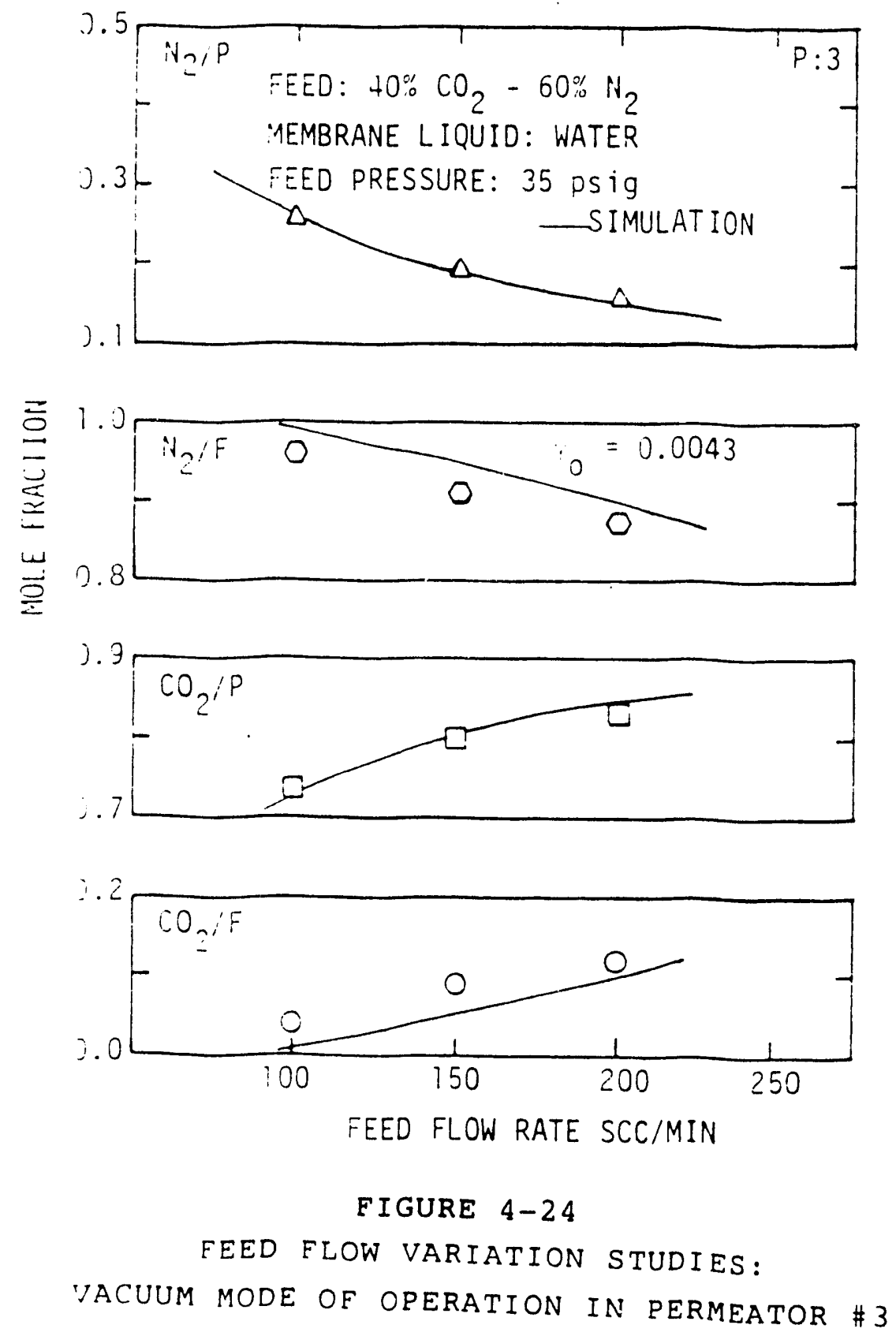



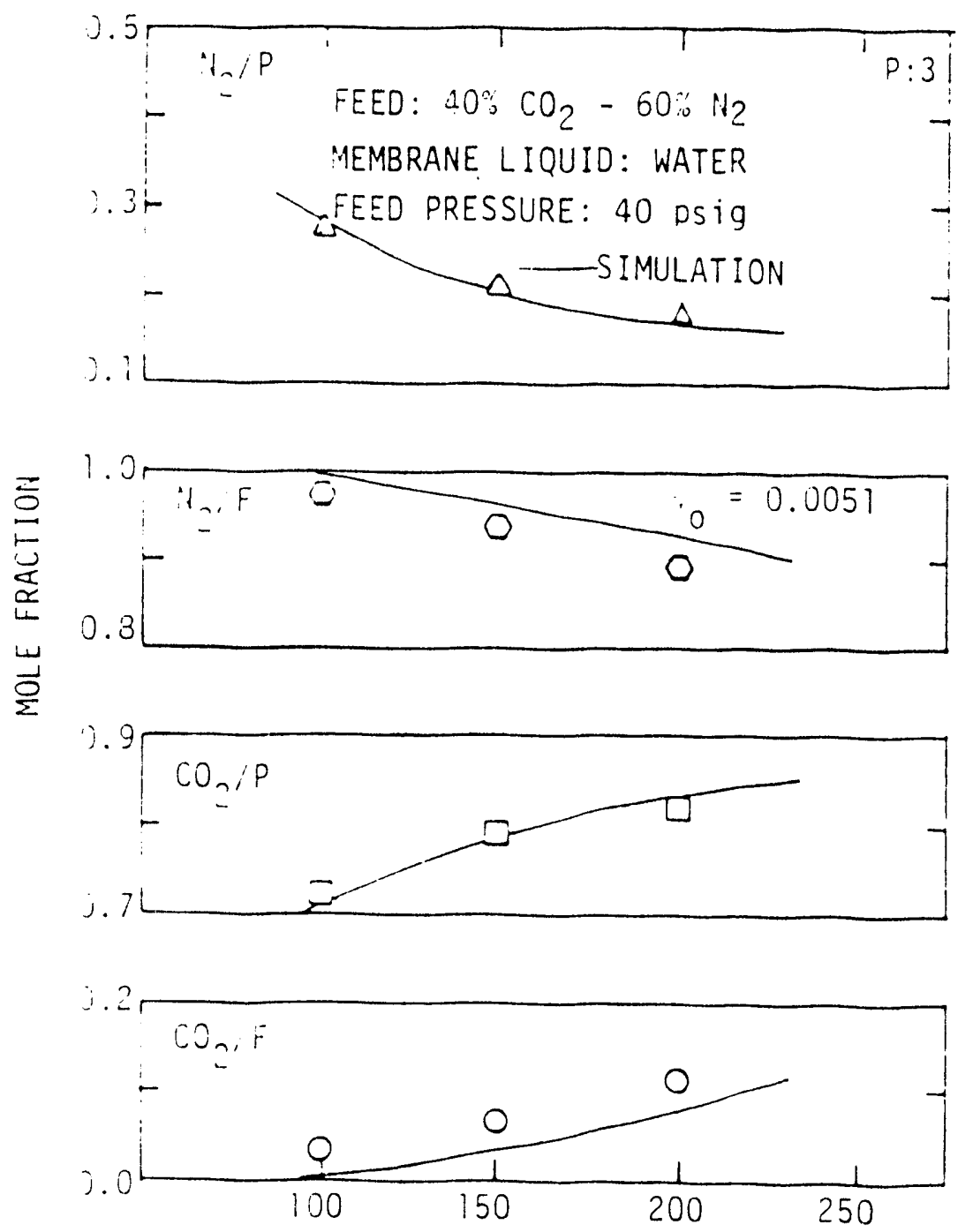

FEED FLOW RATE SCC/MIN

FIGURE $4-25$

FEED FLOW VARIATION STUDIES:

YACUUM MODE OF OPERATION IN PERMEATOR \#3 
feed pressure.

To illustrate the feed pressure effect on separation more clearly, the above three figures are combined in the Figure 4-26. It is evident that higher feed pressure improves the quality of the purified biogas. The simulation results appear to describe the behavior quite well. The $\mathrm{CO}_{2}$ content in the feed outlet is somewhat underpredicted. That is especially true for 40 psig feed pressure.

Similar experiments under vacuum mode of operation were also carried out with permeator \#5. Permeators \#3 and \#5 contain equal number of hollow fibers of identical size and length. However, there is no extra shell space around the fiber bundle in permeator \#5. Feed inlet pressures of 30 and 35 psig were maintained during the experimental runs. The steady state data, obtained by varying the feed flow rates, are reported in Table 4-40.

The experimental data and numerical simulations for feed pressures of 30 psig and 35 psig are shown in Figures 4-27 and 4-28, respectively. The conclusions drawn earlier for permeator \#3 are also valid in this case. The simulation results, in general, agree with the data. The simulation results for permeator \#3 are also valid for permeator \#5 because the effective liquid membrane thicknesses in these two permeators are considered equal.

The performance data for the mixed fiber permeator \#6 are reported in Table 4-41. Vacuum was pulled through the larger fiber set and the permeate gas flow was countercurrent to the feed gas flow. The data for these three different feed pressures are plotted in Figures 4-29, 4-30 and 4-31, respectively. The numerical simulations were carried out with a membrane thickness of 0.01 inch. Here again, we notice that the stage cut increased with a decrease in pressure ratio (ratio of permeate pressure to the feed pressure). At a low feed flow rate (e.g., $50 \mathrm{sccm}$; feed pressure 35 psig) the purified gas stream contained about $11 \% \mathrm{CO}_{2}$. This performance is reasonably good when we consider that permeator \#6 is only $2 \mathrm{ft}$ long and the membrane area in permeator "' is only $40 \%$ of that of permeators \#3 or \#5.

The vacuum purification data for permeator \#8 with pure water as liquid membrane is given in Table 4-42. The data and the numerical results are plotted 

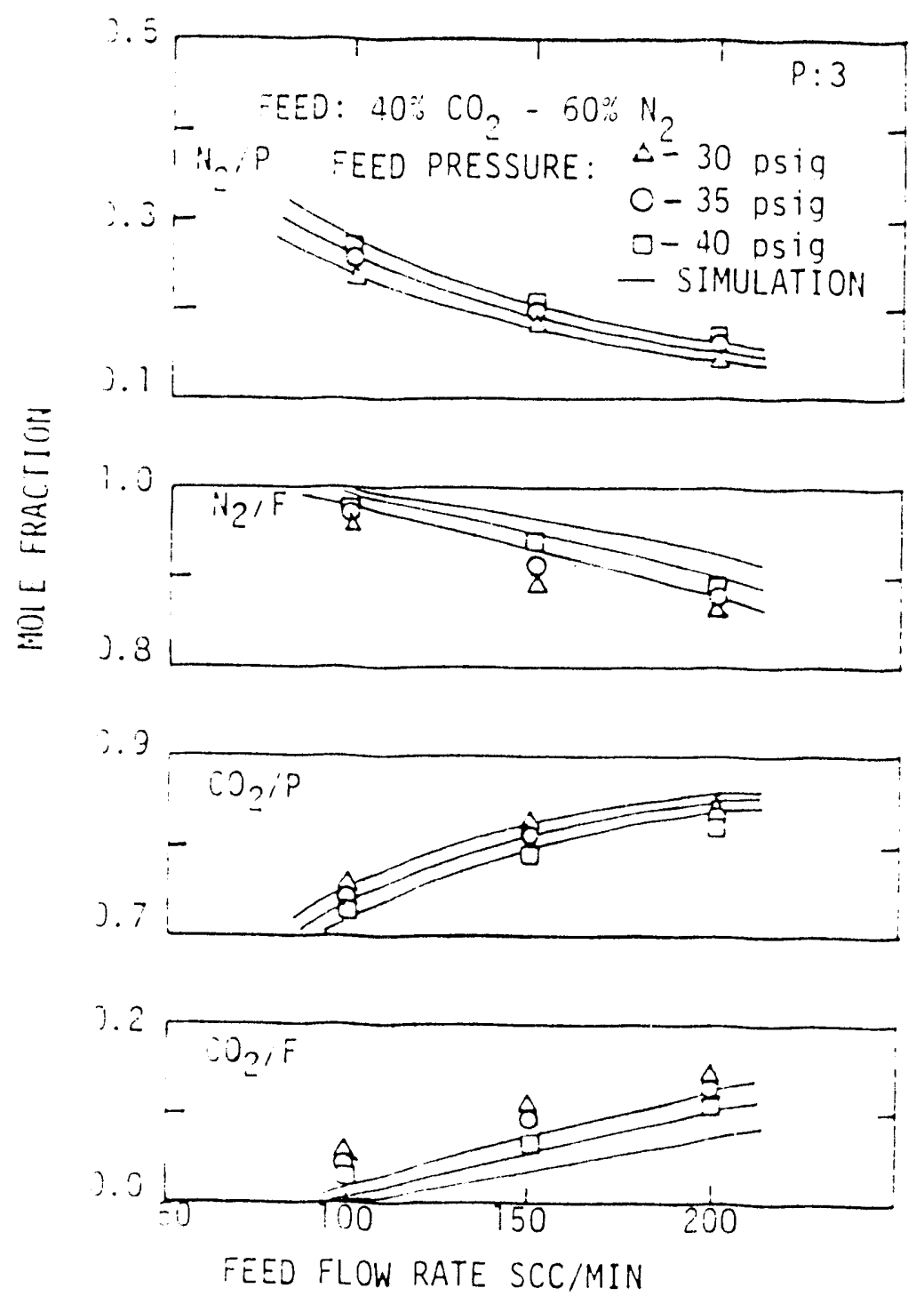

FIGURE $4-26$
FEED FLOW AND PRESSURE VARIATION STUDIES:
VACUUM MODE OF OPERATION IN PERMEATOR $\# 3$ 


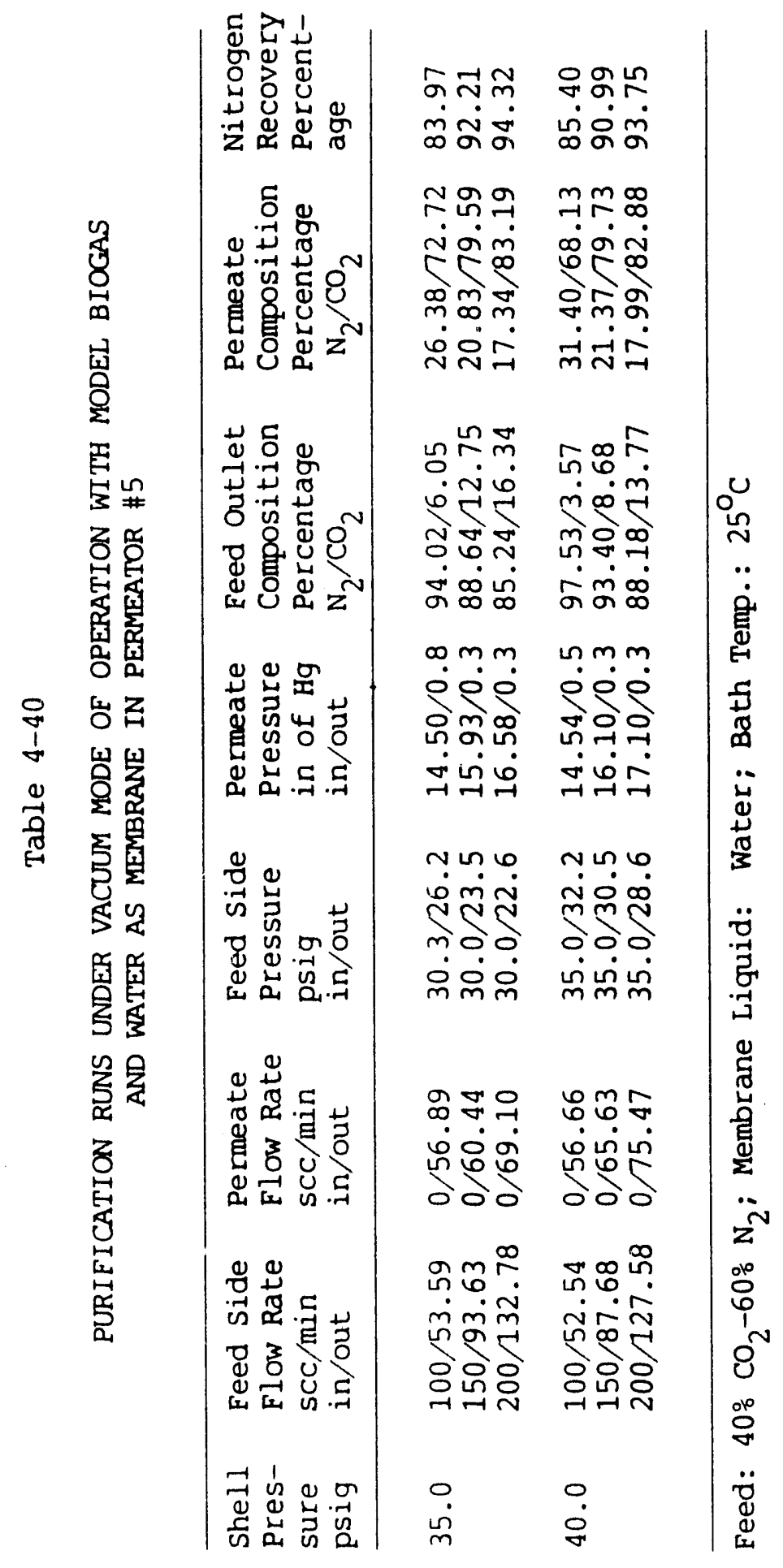




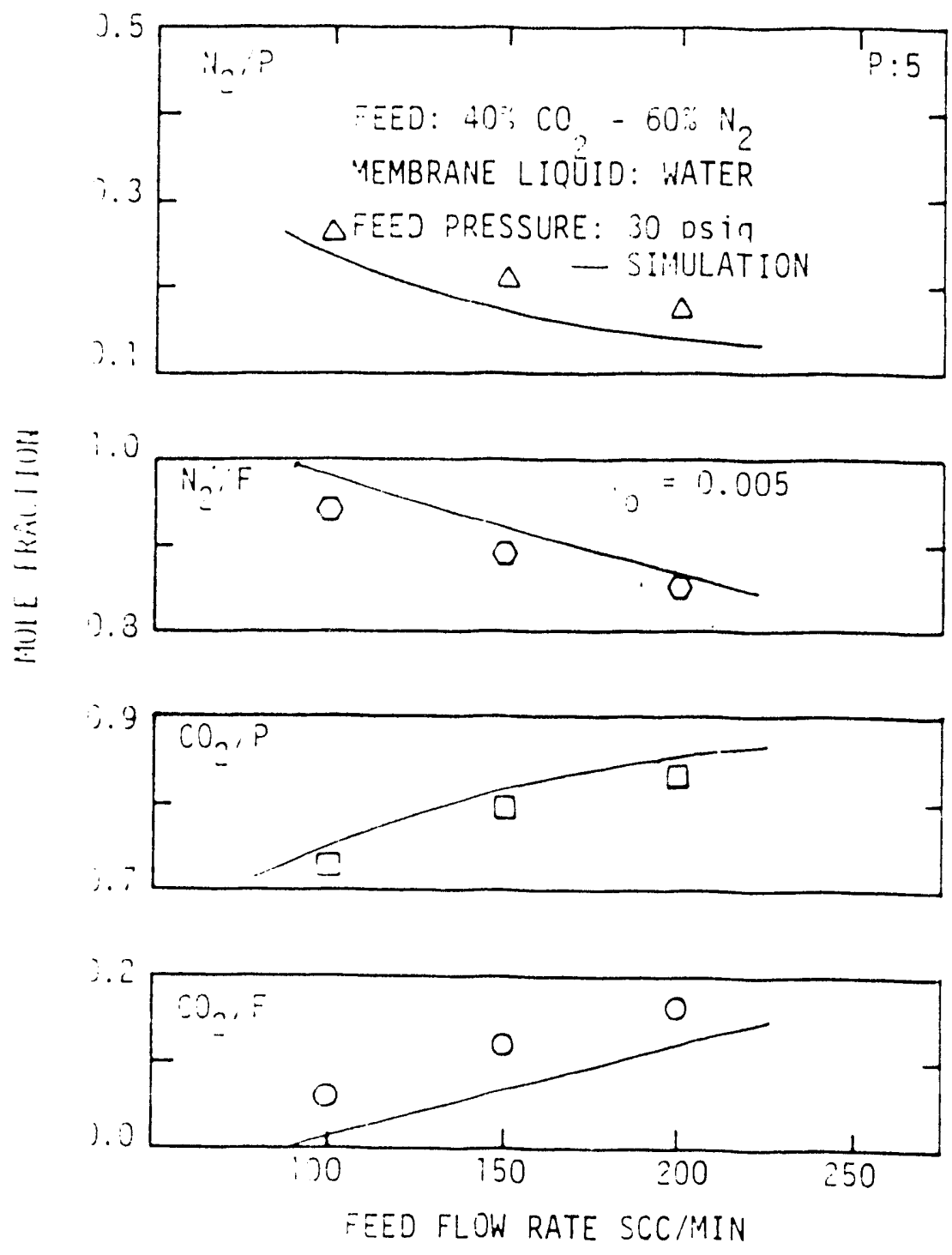

FIGURE $\quad 4-27$

PEED FLOW VARIATION STUDIES:

AIIIU MODE OF OPERATION IN PERMEATUR \#5 

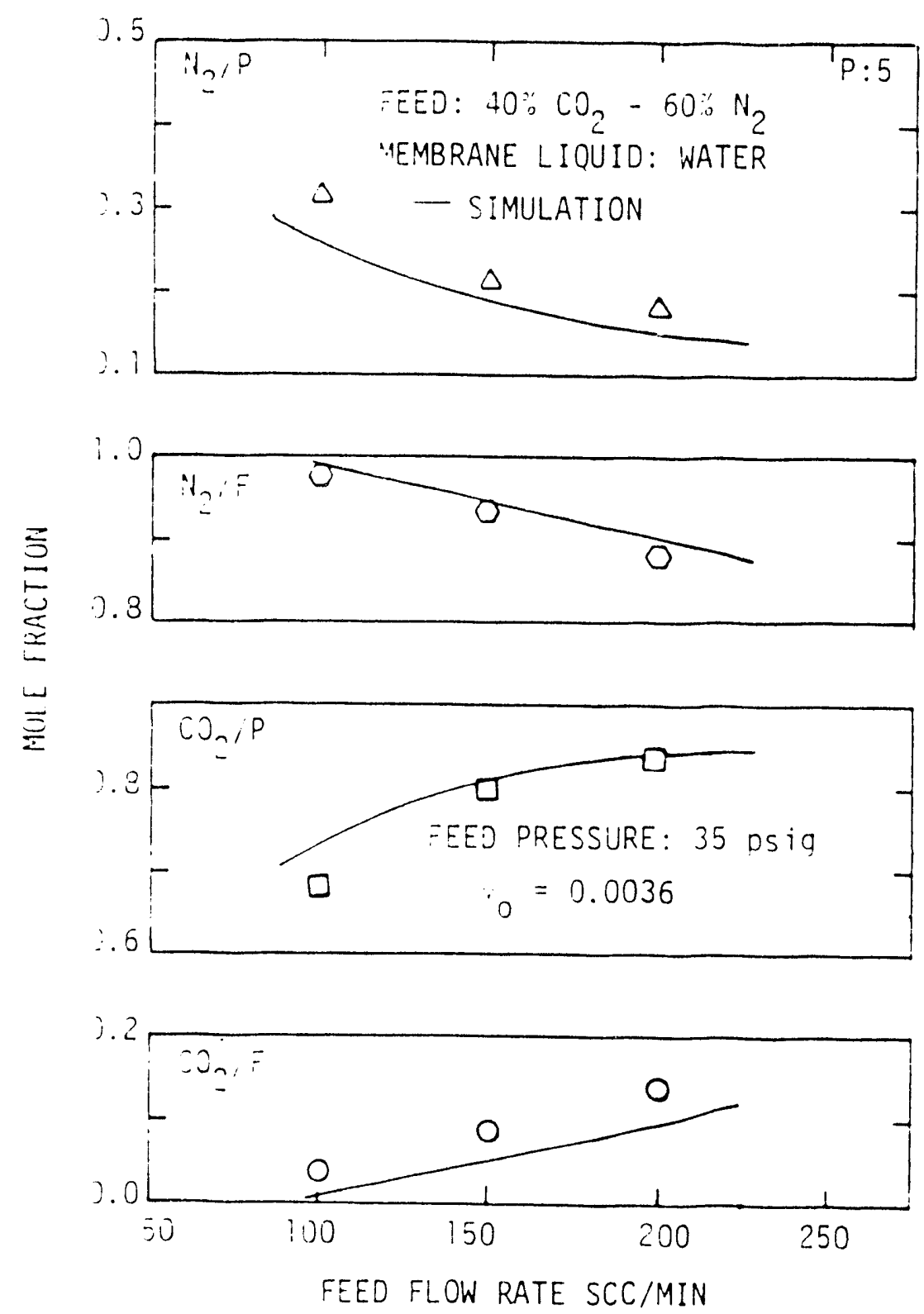

FIGURE $4-28$

FEED FLOW VARIATION STUDIES:

VACUUM MODE OF OPERATION IN PERMEATOR \#5 


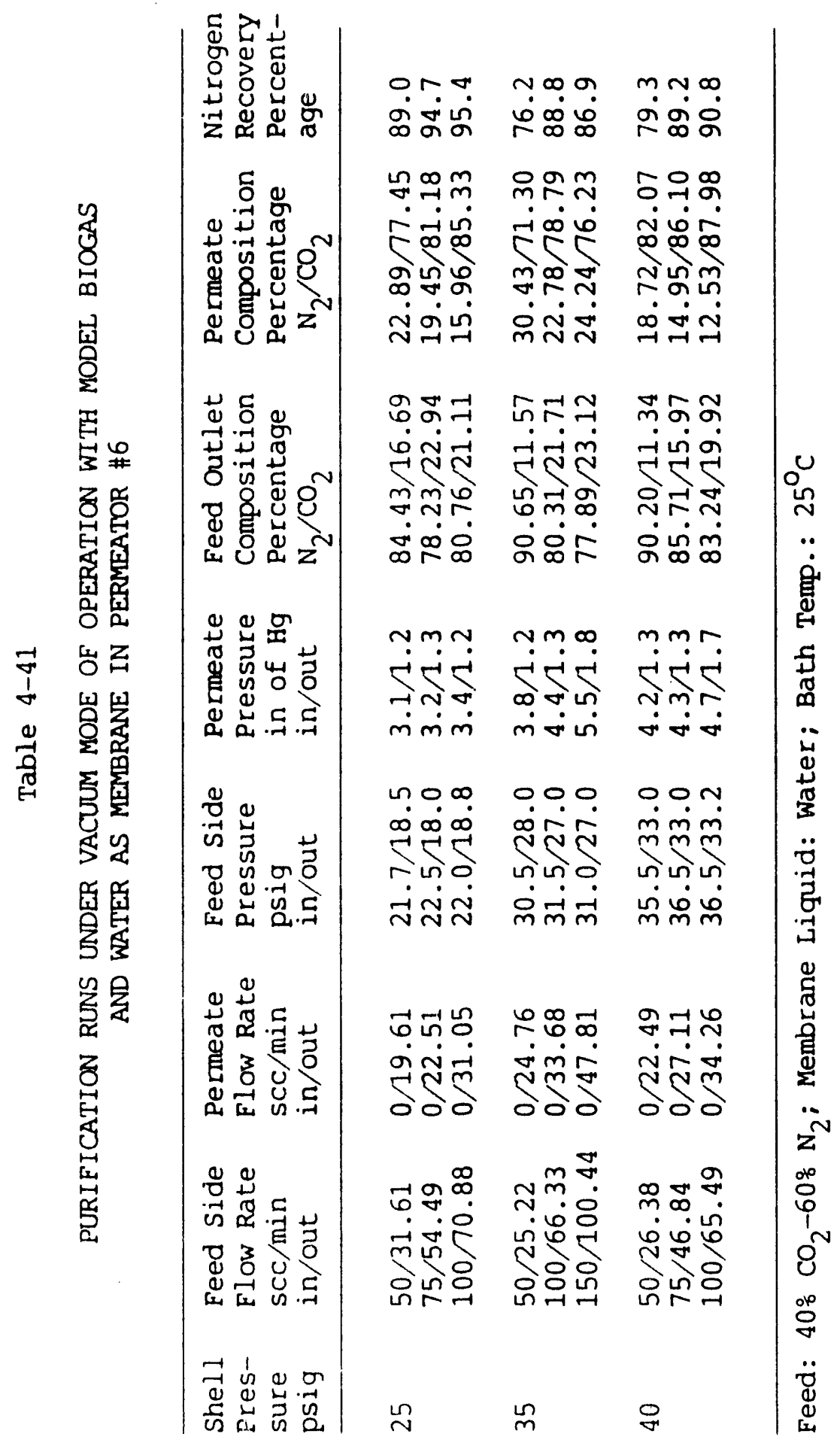



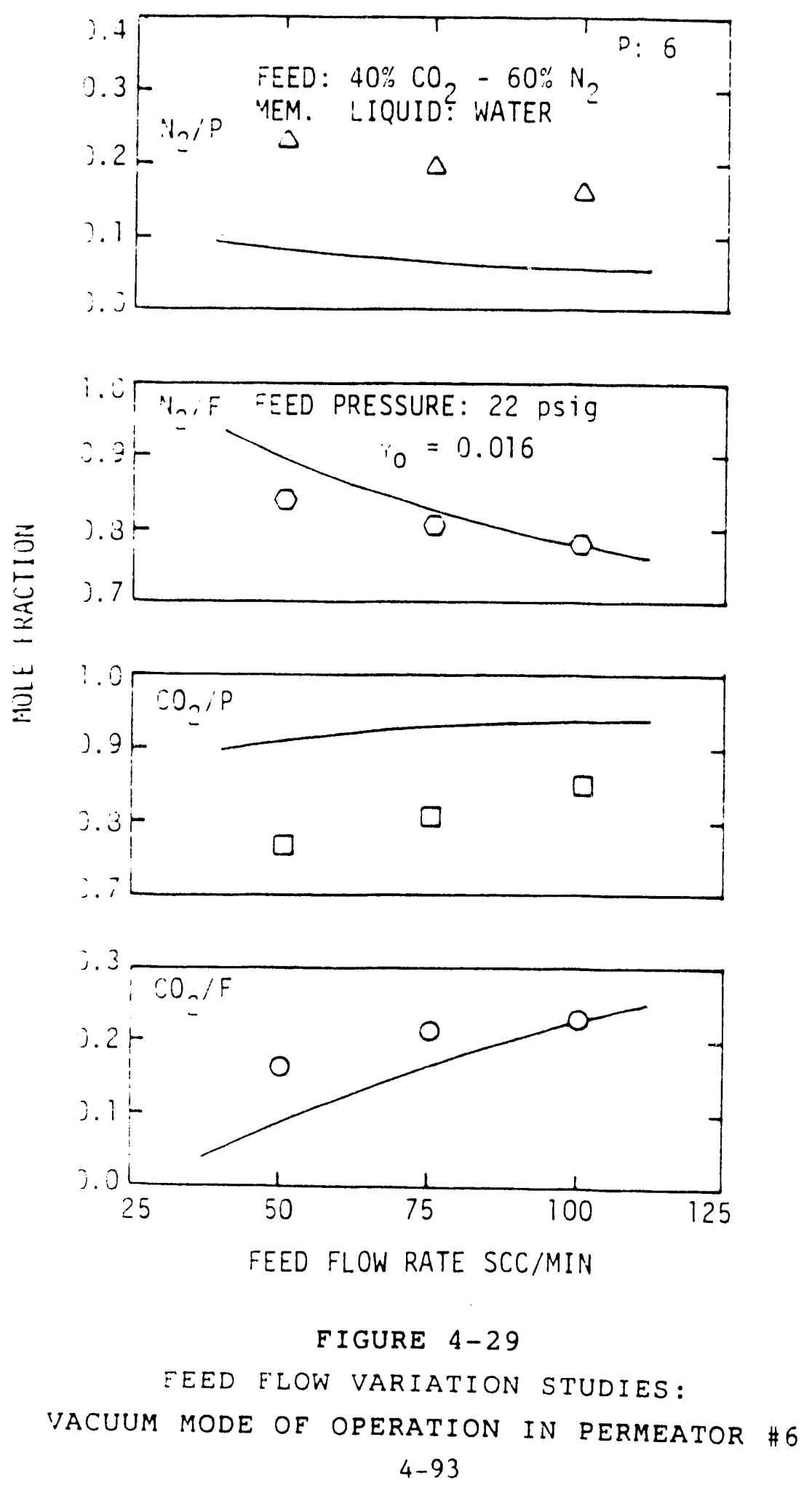


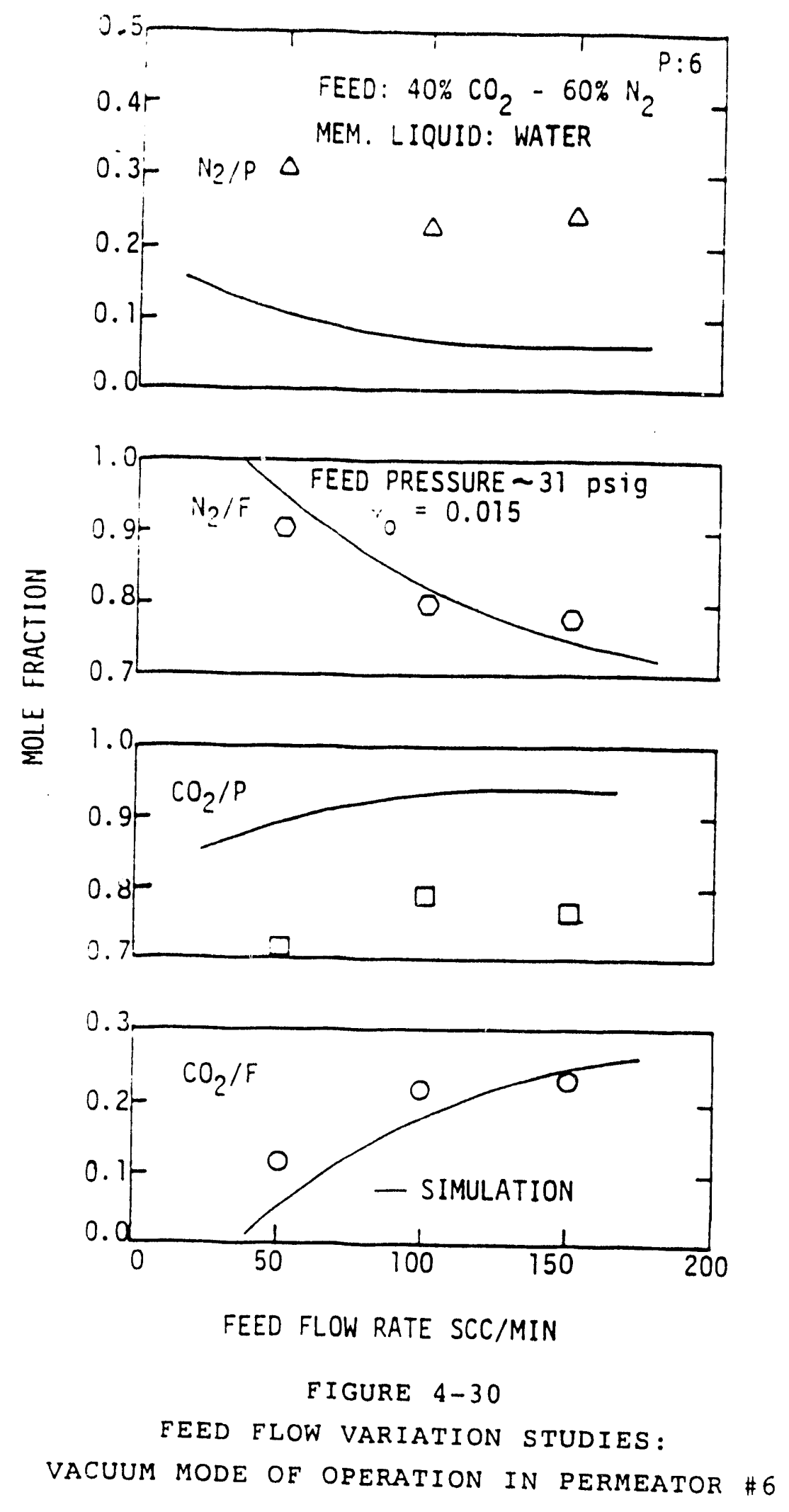



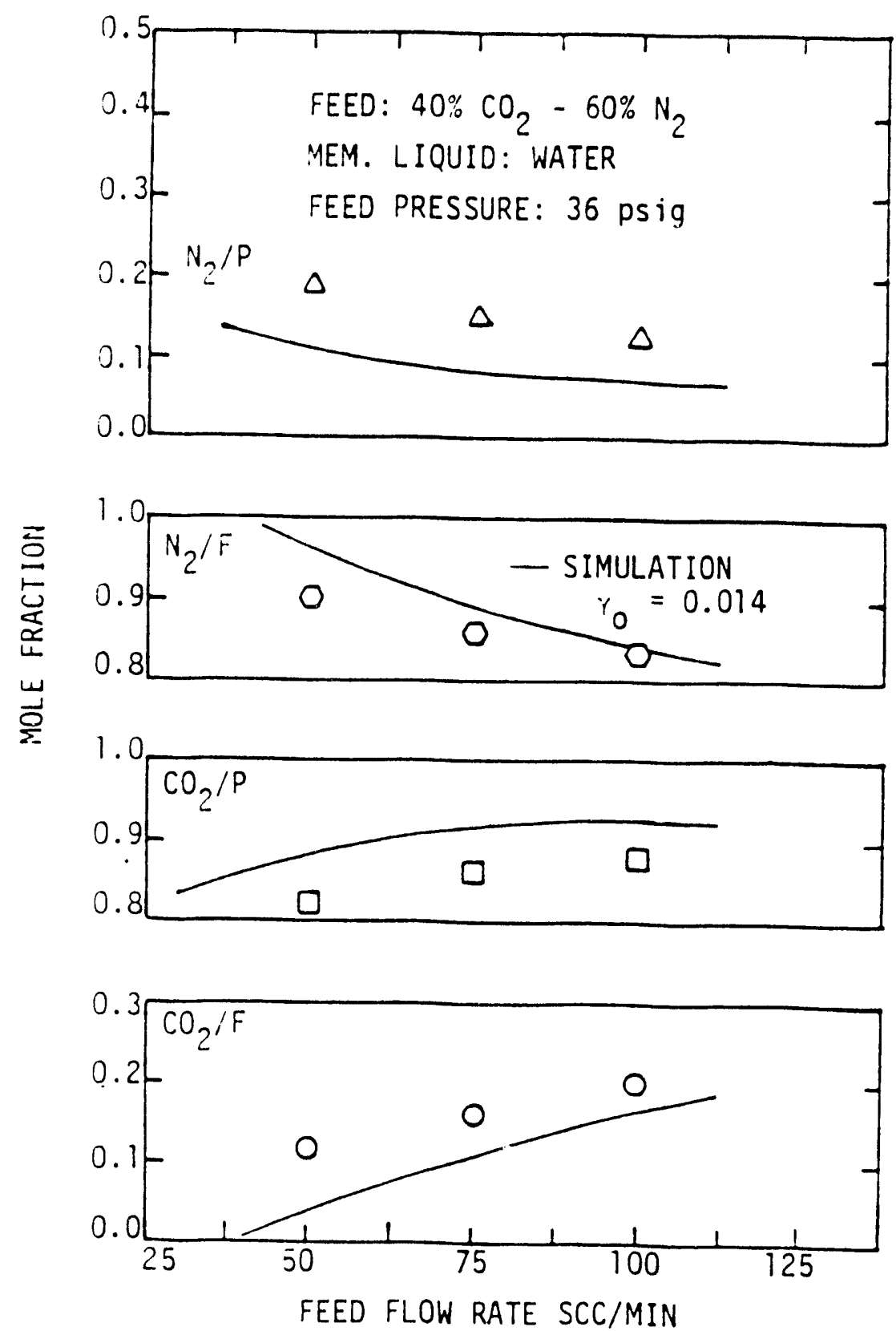

FIGURE 4-31

FEED FLOW VARIATION STUDIES:

VACUUM MODE OF OPERATION IN PERMEATOR \#6 


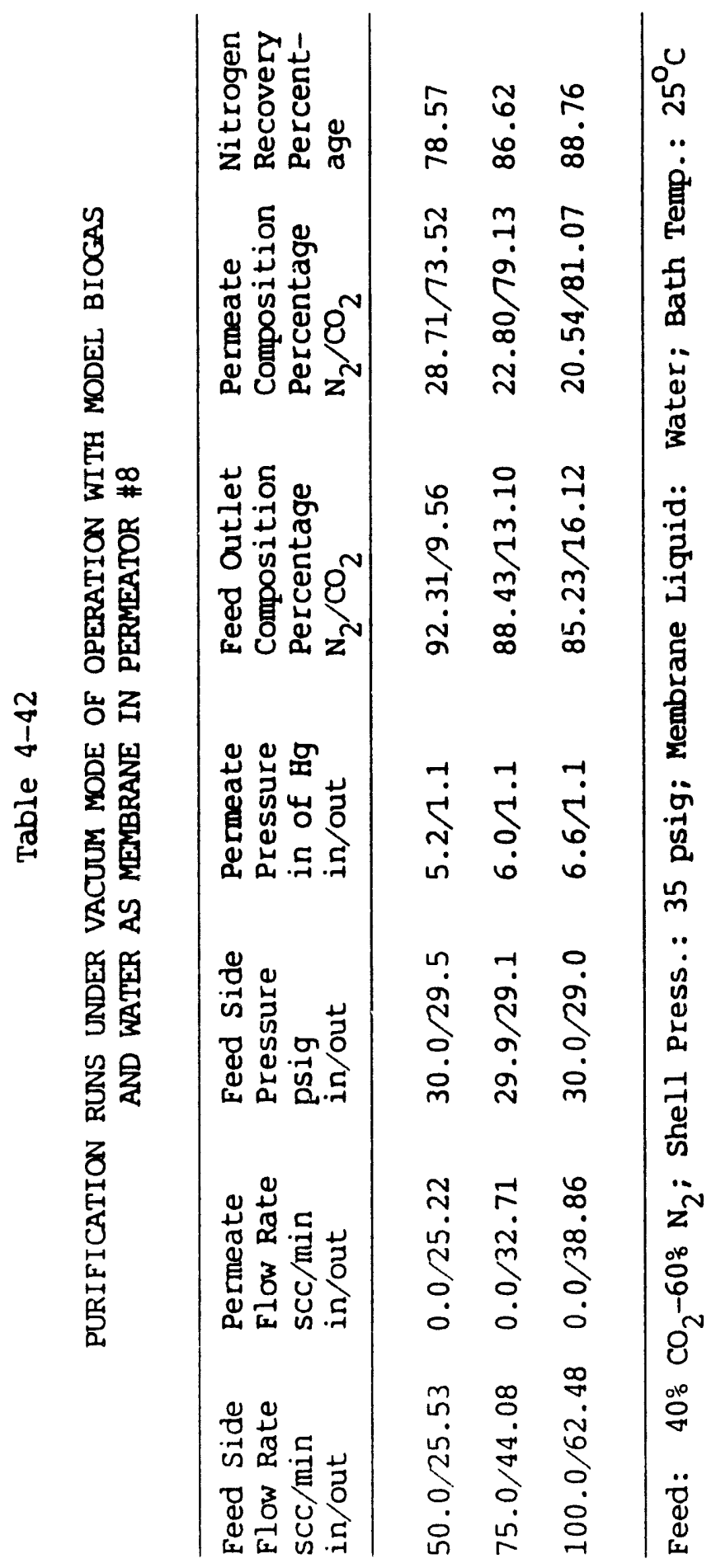


in Figure 4-32. The numerical results were obtained with a membrane thickness value of 0.00485 inch. The performance of permeator \#8 was better than that of the mixed fiber permeator \#6. For example, in case of permeator \#8 with 100 $\mathrm{sccm}$ feed flow rate, we found about $16 \% \mathrm{CO}_{2}$ in the purified biogas stream whereas, under identical conditions, the $\mathrm{CO}_{2}$ composition in the purified gas stream was about $22 \%$ when permeator \#6 was used. Note further that, permeator \#8 r.as smaller membrane area than permeator \#6. The bettur performance of permeator \#8 was most likely due to a lower effective liquid membrane thickness in permeator \#8. The reduction in pressure drop in the large bore permeate fihers apparently has very limited effect for high feed gas pressure situations in the vacuum mode.

In order to explore liquid membrane stability under the vacuum mode of operation, a few long-term experiments were also carried out. Table 4-43 shows such a run carried out with permeator \#3. Steady state was achieved within a period of 3-4 hrs, and all the parameters were maintained constant over the 26hr. period after which the feed flow rate was changed to $150 \mathrm{sccm}$. Results of a similar experiment, carried out with permeator \#5, are reported in Table 4-44 and the performance is shown in Figure 4-33. The purified gas stream showed only $3.5 \% \mathrm{CO}_{2}$ and remained constant for the entire period. The nitrogen recovery was about $85 \%$.

In all such experiments, vacuum was pulled through one end of the permeate fibers while the other end was closed. In a variation of this simple vacuum mode, vacuum was pulled through the both ends of the permeate fibers of permeator \#5. This mode was identified as split vacuum mode (Majumdar, 1982; Sengupta and Sirkar, 1986). The stab]? behavior is shown in Table 4-45. The data also show a slight improvement is permeation rate and qualit; of the purified gas compared to the simple vacuum mode operation (3rd row; Table $4-40)$.

Water as Liquid Membrane and $40 \% \mathrm{CO}_{2}-60 \% \mathrm{CH}_{4}$ as Feed Gas

Long-term purification study of a $\mathrm{CO}_{2}-\mathrm{CH}_{4}$ feed gas mixture was made under vacuum mode of operation using permeators $\# 6$ and \#7, connected in series. The feed inlet flow rate was initially maintained at $75 \mathrm{sccm}$. The results are 


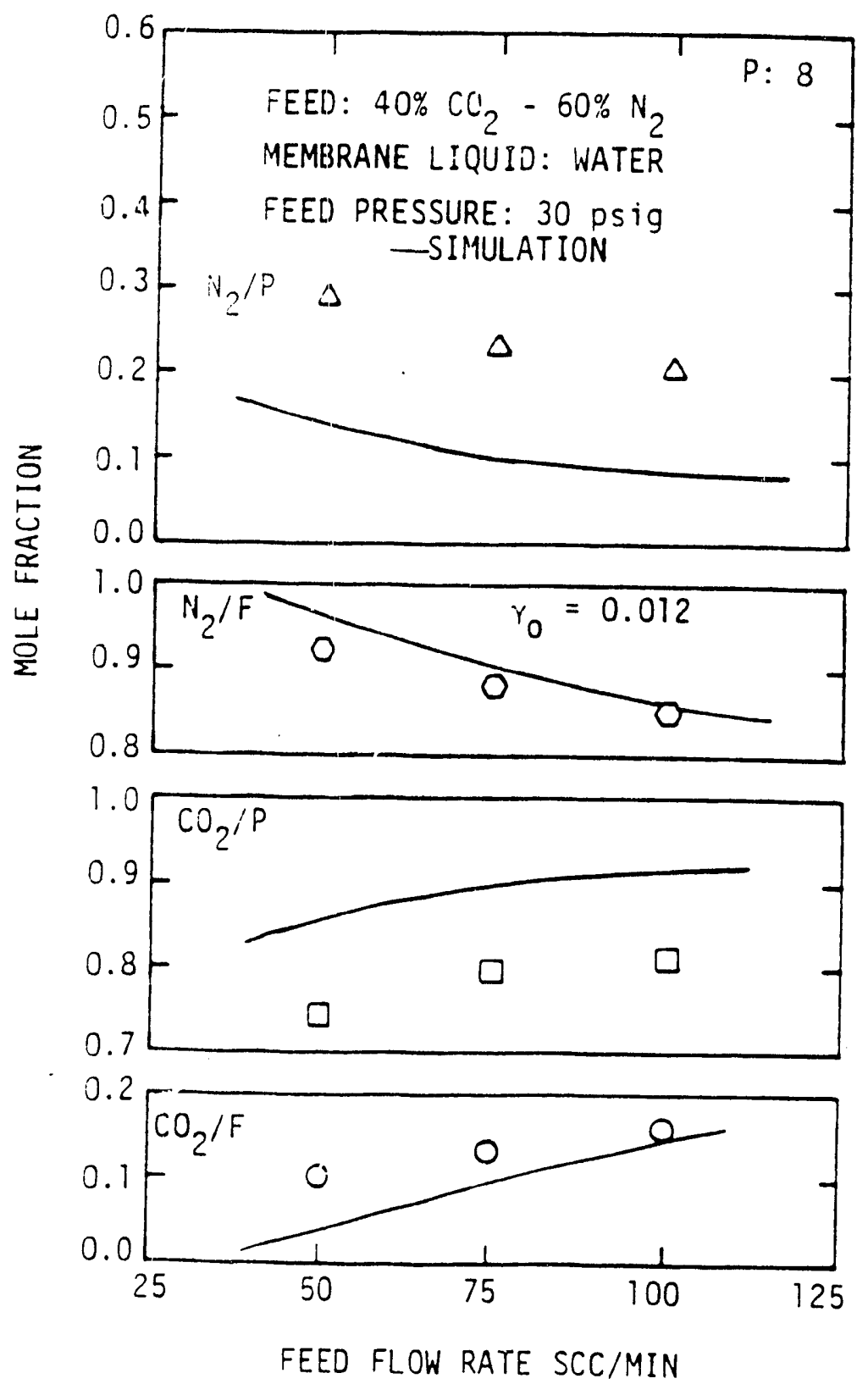

FIGURE $4-32$

FEED FLOW VARIATION STUDIES:

VACUUM MODE OF OPERATION IN PERMEATOR \# 8 4-98 


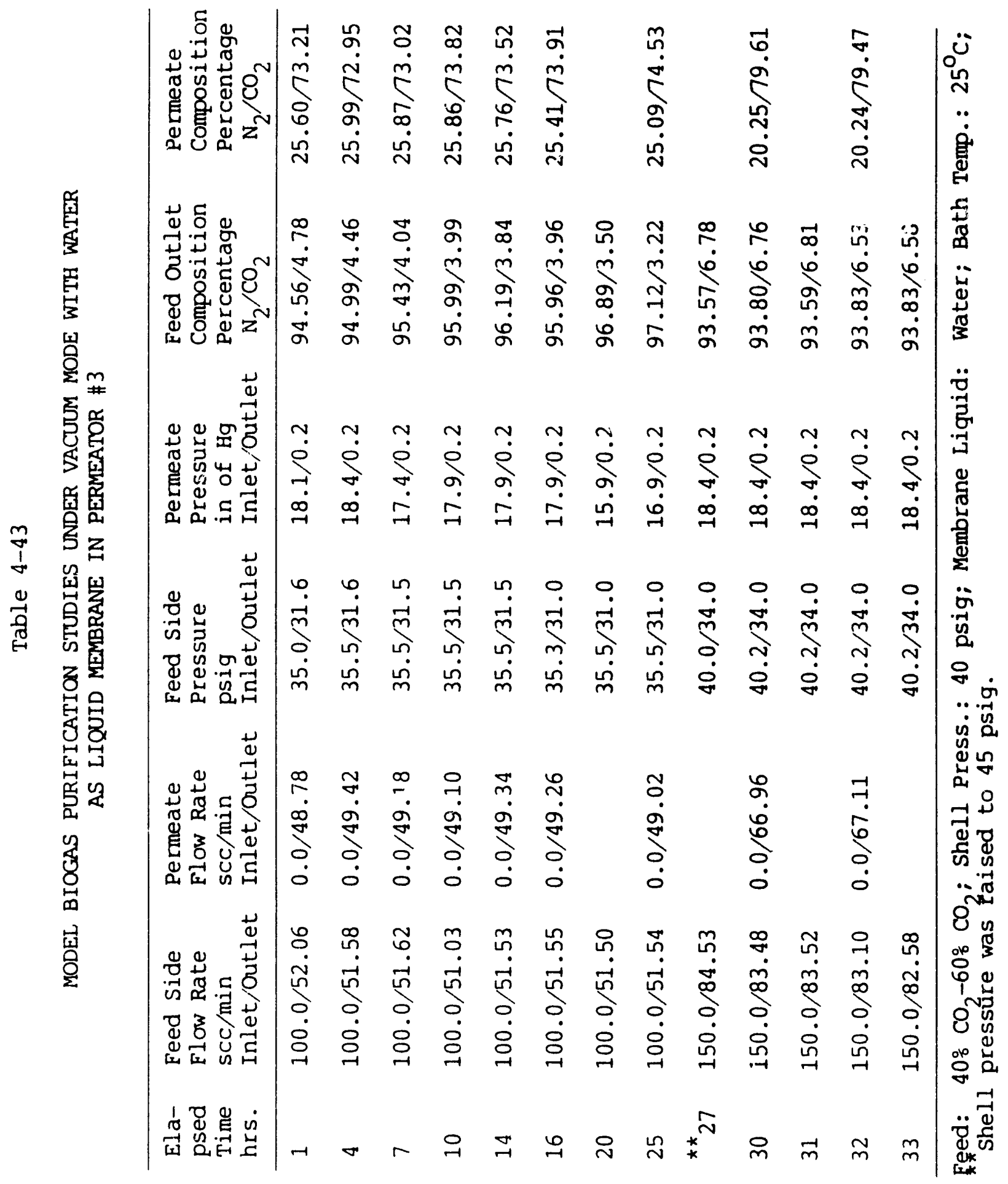




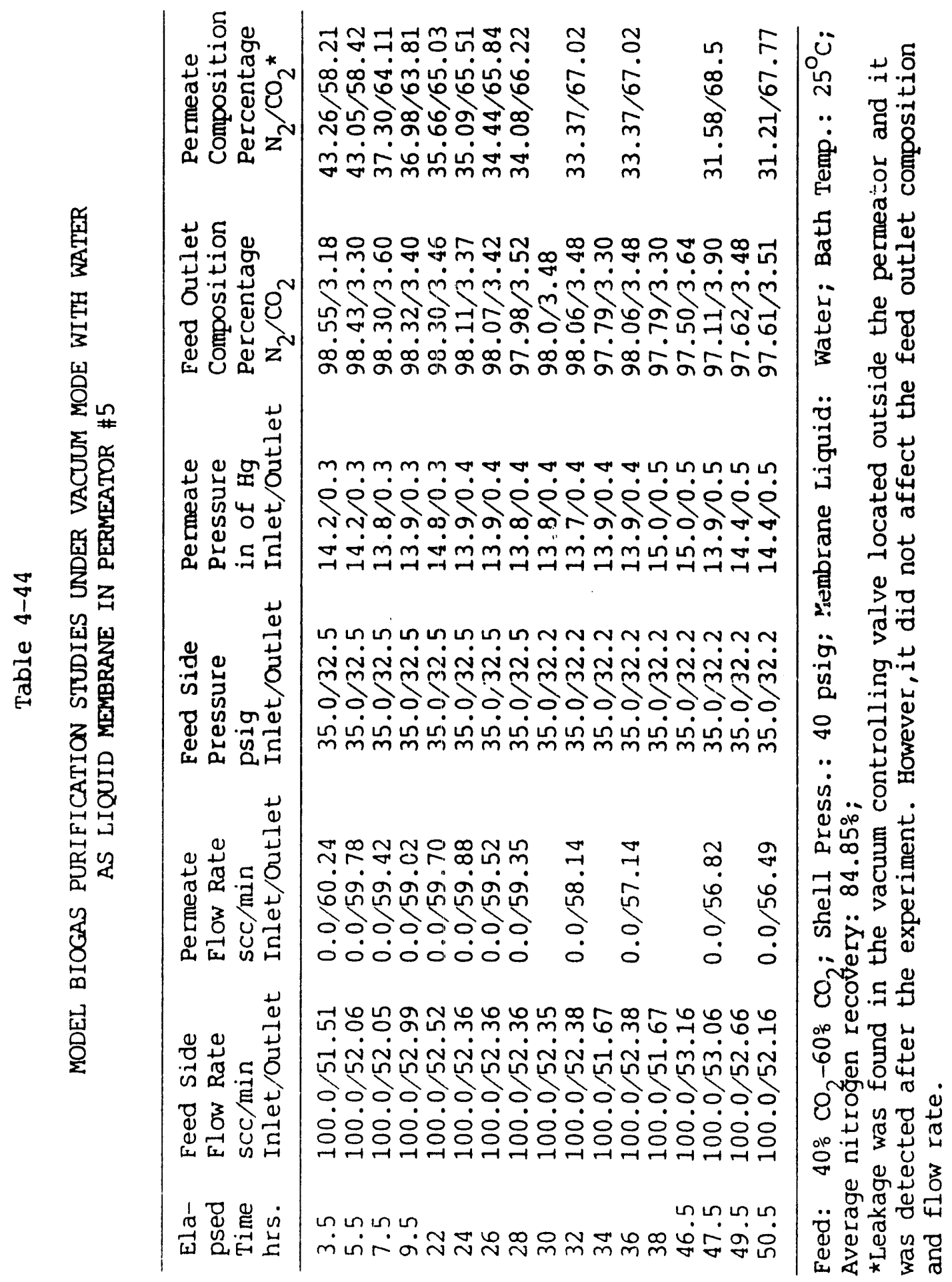




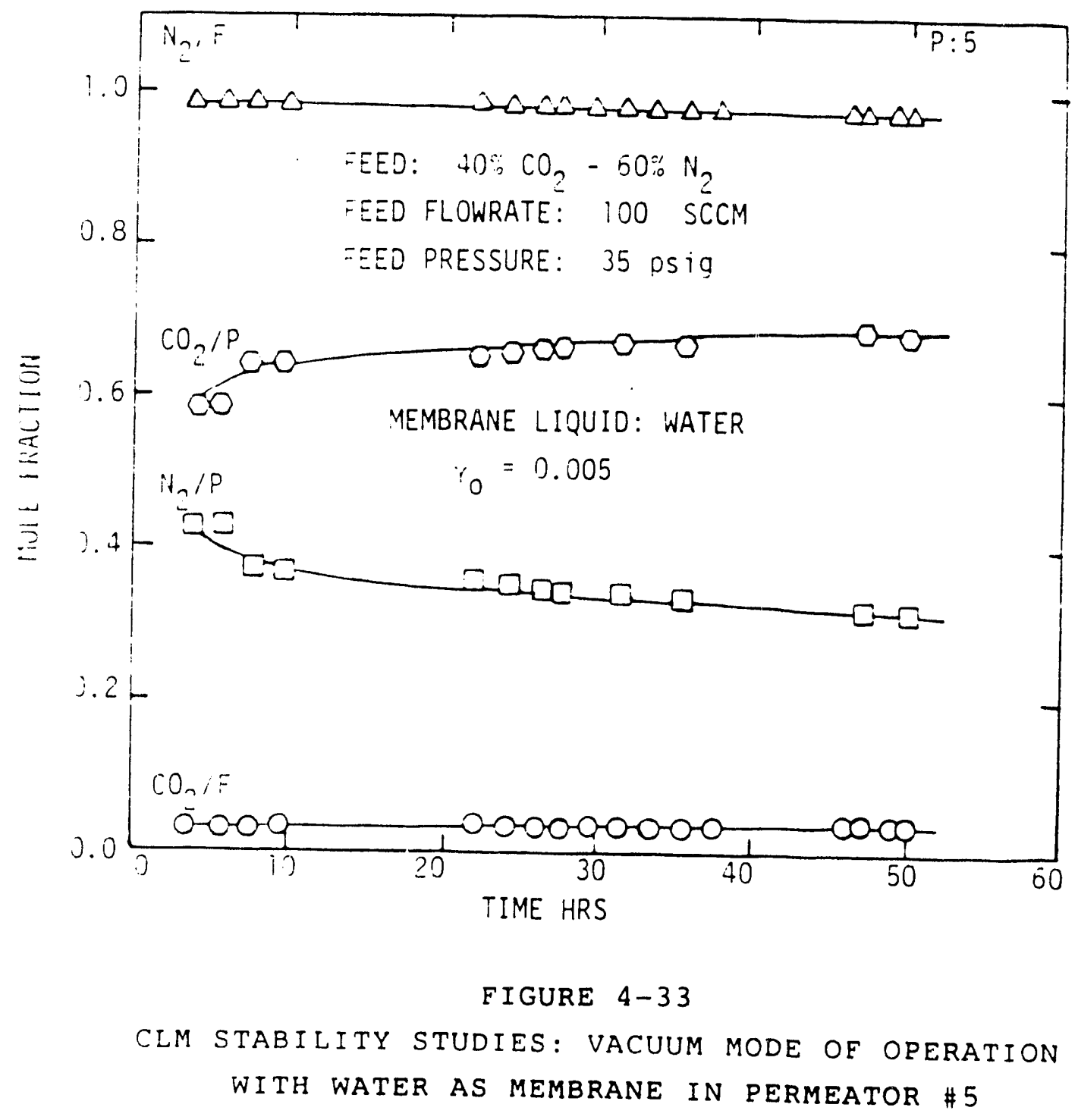




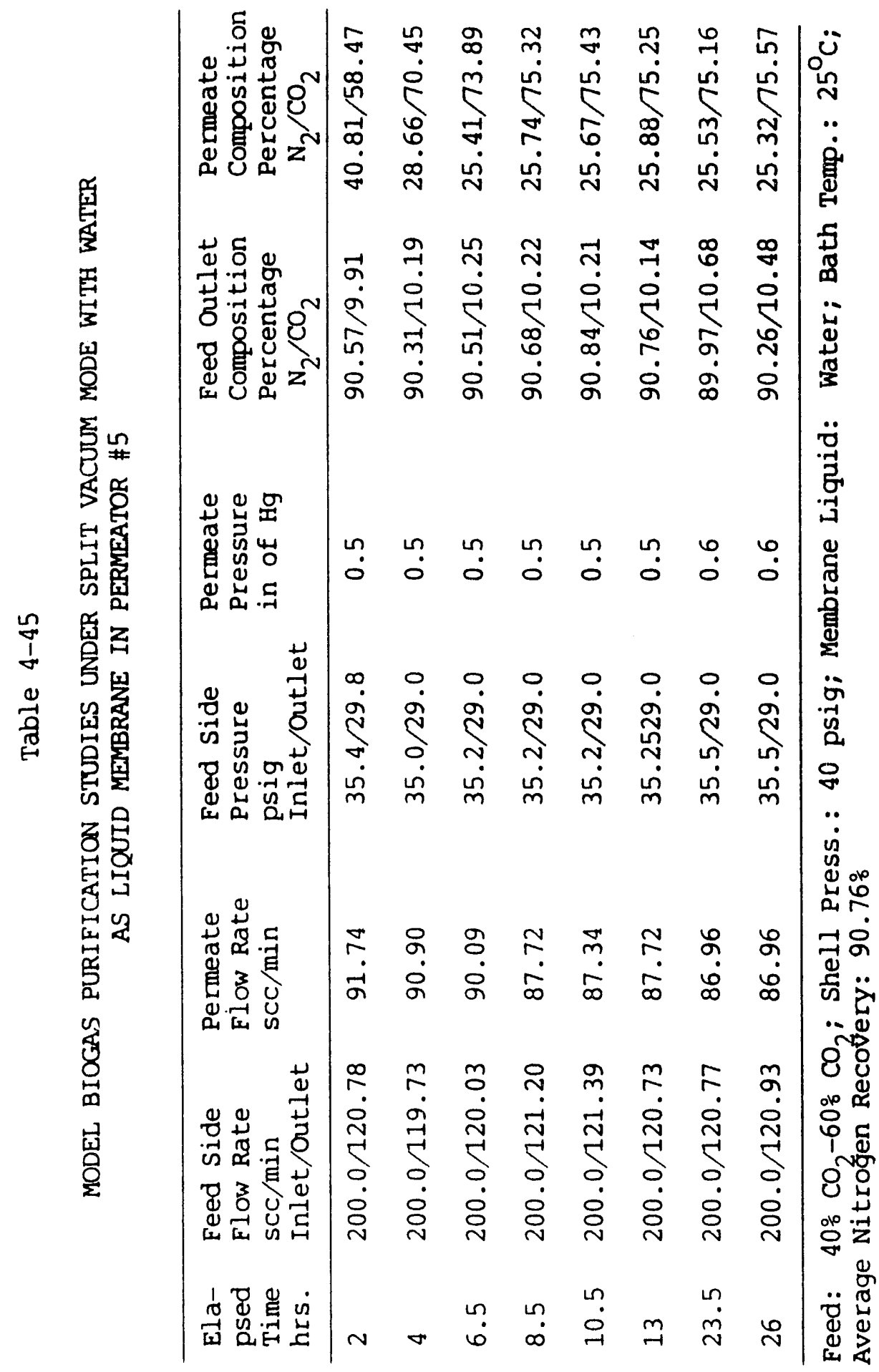


presented in Table 4-46 and in Figure 4-34. The average $\mathrm{CH}_{4}$ recovery for this feed flow rate $(75 \mathrm{sccm})$ was $85.3 \%$. The $\mathrm{CO}_{2}$ composition in the purified gas stream was about $13 \%$. The results also show very little pressure drop in the permeate side. Note that, vacuum was applied through the set of fibers with a larger diameter. After 24 hours of experimental run, the feed gas flow rate was changed to $60 \mathrm{sccm}$. This produced a slightly higher $\mathrm{CH}_{4}$ composition ( $90 \%$ ) at the purified gas end.

The above set of experiments suggest unequivocally that a trouble-free operation is possible with vacuum mode operation when pure water is utilized as a liquid membrane.

7 wt $\% \mathrm{~K}_{2} \mathrm{CO}_{3}$ Solution as Liquid Membrane and $40 \% \mathrm{CO}_{2}-60 \% \mathrm{~N}_{2}$ as Feed Gas

In the next two Tables 4-47 and 4-48, the vacuum purification performances with $7 \% \mathrm{~K}_{2} \mathrm{CO}_{3}$ solution are illustrated. The first set of data were taken with permeator \#3 which has a large shell space around the fiber bundle. Here, the feed outlet behavior with respect to $\mathrm{CO}_{2}$ showed some unsteadiness. This unsteadiness was somewhat eliminated when permeator \#4 was used (Table 4-48). However, the quality of the purified gas was poor even when the feed flow rate was reduced to a lower value of $50 \mathrm{sccm}$ (Table 4-48).

\section{PURIFICATION STUDIES OF MODEL BIOGAS MIXTURES IN SWEEP WATER MODE} THROUGB DIFFERENT LIQUID MEMBRANES

Model biogas purification studies were carried out in sweep water mode with different liquid membranes such as pure water, aqueous $30 \mathrm{wt} \% \mathrm{~K}_{2} \mathrm{CO}_{3}$ solution and aqueous 20 wt\% DEA solution. Pure water was used as sweep liquid for purification of model biogas since $\mathrm{CO}_{2}$ solubility in water is considerably higher than that of $\mathrm{CH}_{4}$ or $\mathrm{N}_{2}$. Further, it may significantly reduce the cost of pumping the sweep stream. Nonhumidified feed gas was used in all experiments.

Water as Liquid Membrane and $40 \% \mathrm{CO}_{2}-60 \% \mathrm{~N}_{2}$ as Feed Gas

The steady state experimental data as well as simulation results using permeator \#6 are presented in Table 4-49. Water was introduced through the 


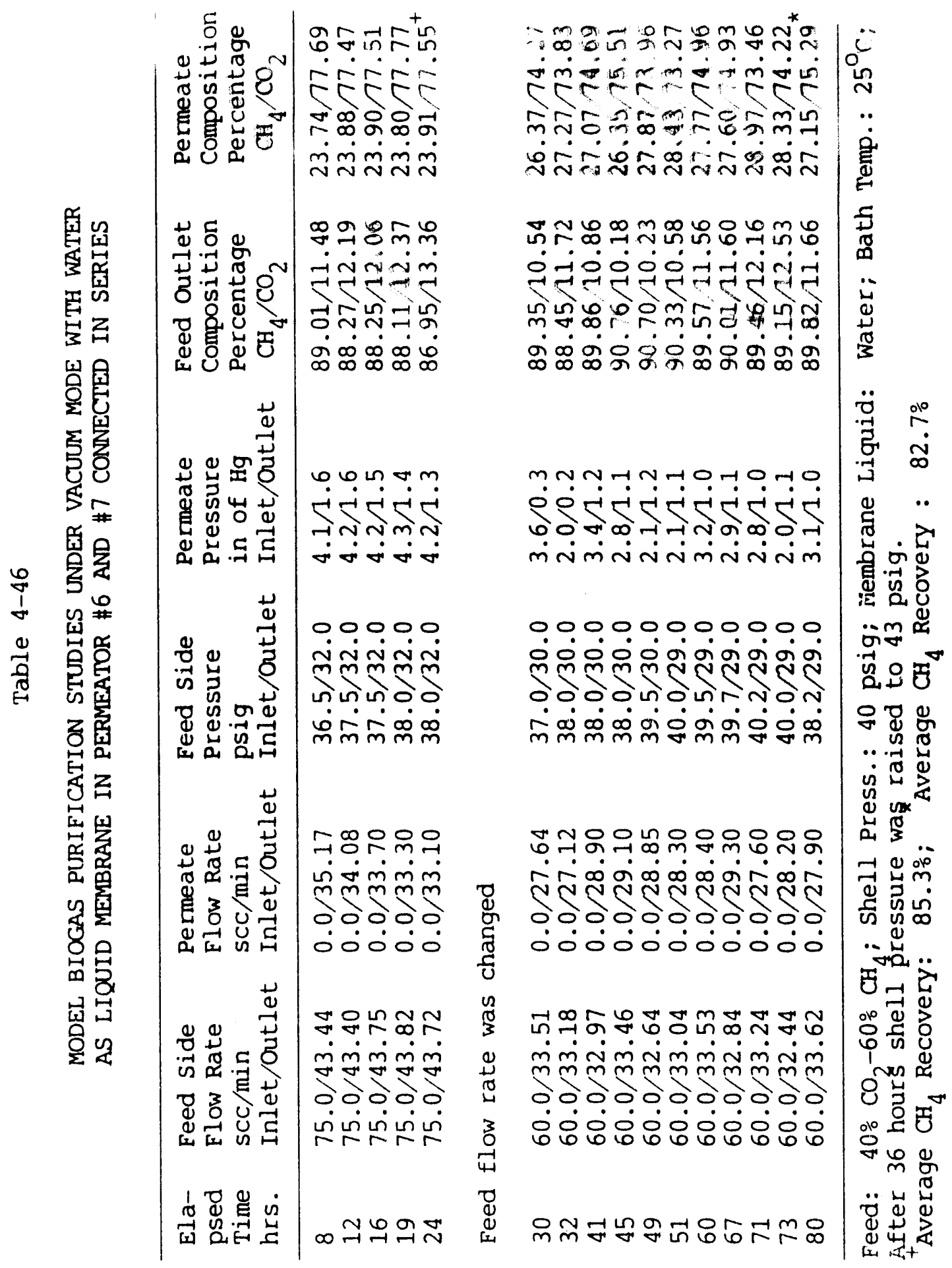




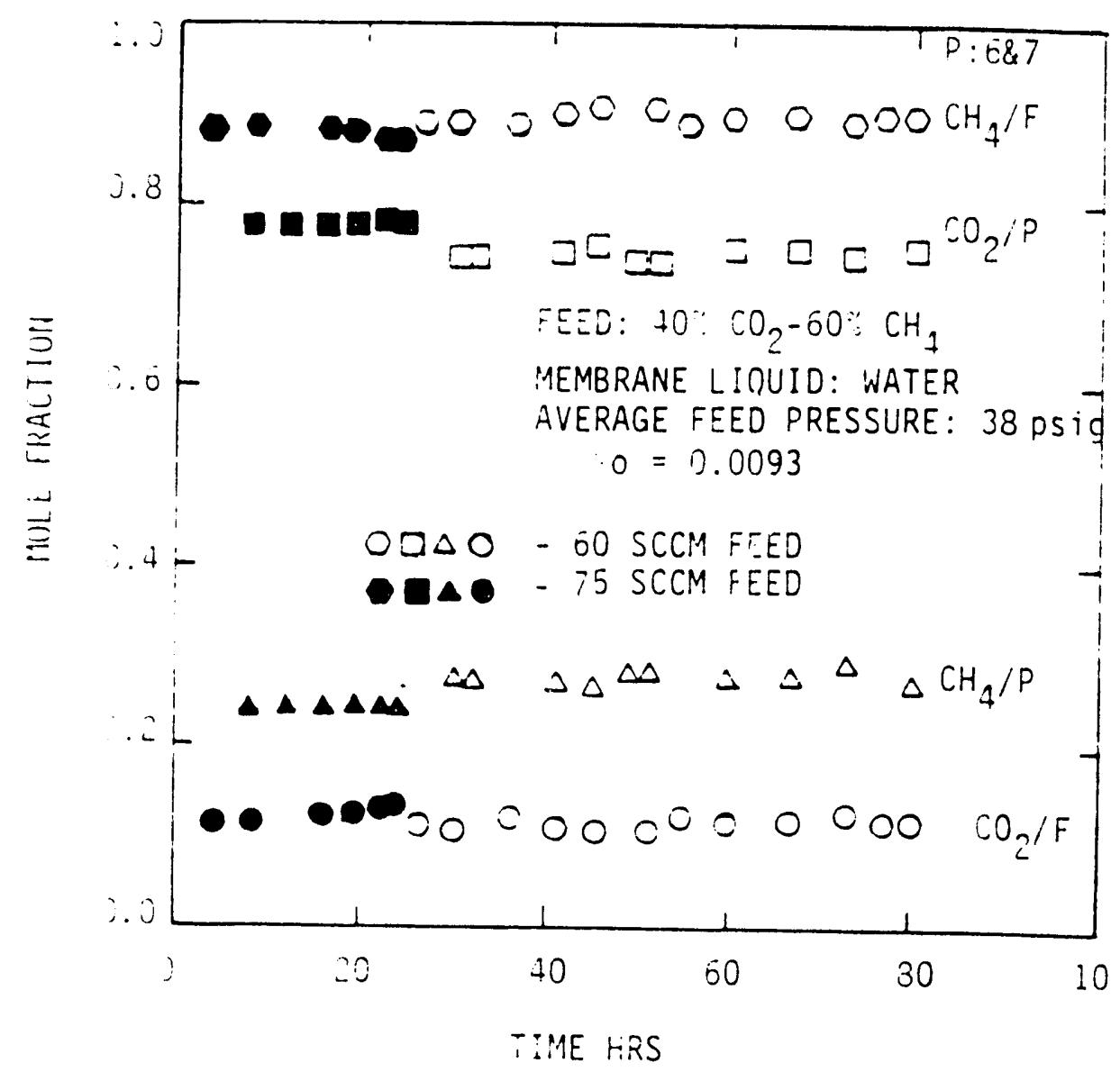

FIGURE $4-34$

CLM STABILITY STUDIES: VACUUM MODE OF ORERATION WITH WATER AS MEMBRANE USING PERMEATORS \#6 AND \#7 CONNECTED IN SERIES 


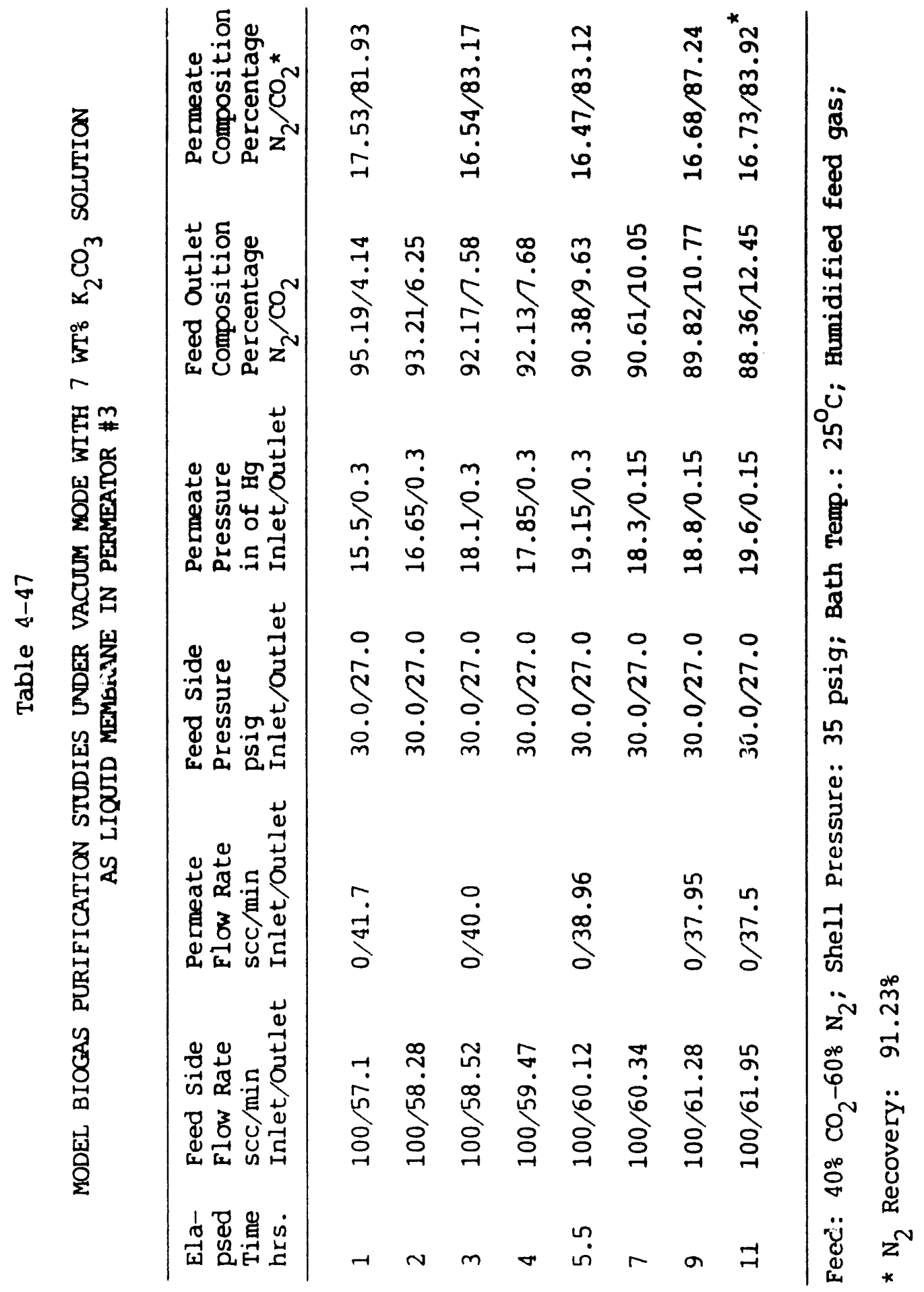




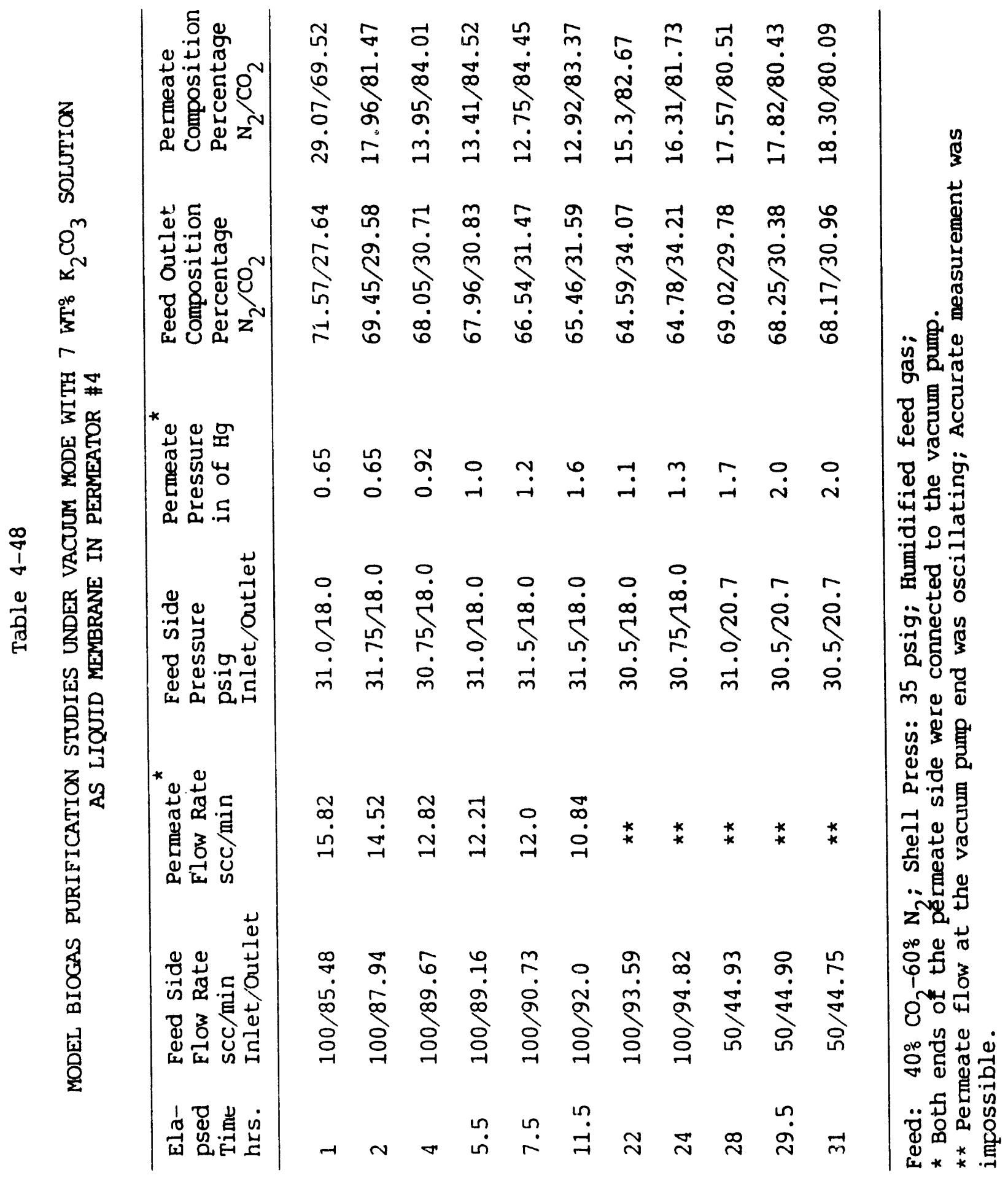




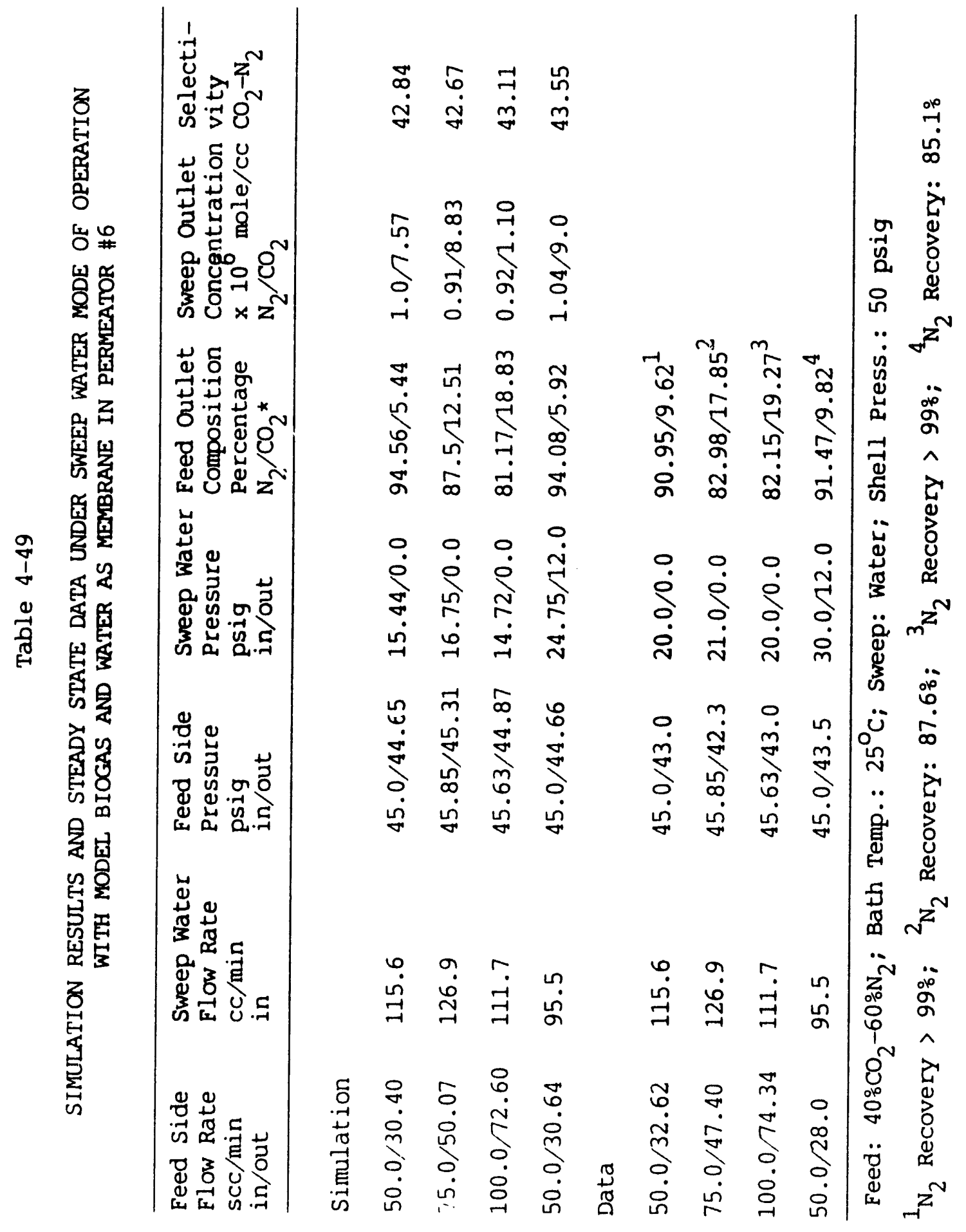


larger diameter fibers of permeator \#6 to absorb the $\mathrm{CO}_{2}$ enriched permeate gas as it is expected that pressure drop would be much less compared to the smaller diameter fibets. The sweep water outlet pressure was maintained at one atmosphere for the first three runs. The purification results are highly encouraging.

At $50 \mathrm{sccm}$ feed flow rate, the purified gas streams contain about $10 \% \mathrm{CO}_{2}$. The $\mathrm{N}_{2}$ recovery was very high. The pressure drop in the sweep line was not very high. For a sweep flow rate of about $115 \mathrm{cc} / \mathrm{min}$, the pressure drop was approximately $20 \mathrm{psi}$. The data show that the $\mathrm{CO}_{2}$ composition in the purified biogas increased with an increase in feed inlet gas flow rate.

In the fourth experiment, sweep water side pressure was raised by using a back pressure regulator to study its effect on separation. The sweep inlet and outlet pressures were maintained at $30 \mathrm{psig}$ and $12 \mathrm{psig}$, respectively. The feed gas flow rate was kept at $50 \mathrm{sccm}$. Comparing this set of data with that of first one it is obvious that the magnitude of sweep pressure did not affect the purified gas composition. However, the rate of permeation was slightly higher in this case. Correspondingly, the $\mathrm{N}_{2}$ recovery was lower. Note that, the flow rate of water is lower in this case. The numerical simulation results are in reasonable agreement with the data.

Purification of model biogas was also carried out in this mode using permeator \#8 at two different feed flow rates. The steady state data and simulation results are shown in Table 4-50. Sweep water side pressure was higher compared to permeator \#6 because of the smaller fiber diameter.

The higher $\mathrm{N}_{2}$ recovery in the sweep water mode can be explained in the following way. The mass transfer of a solute in this configuration can be shown schematically in Figure 4-35. Any permeable species has to face three resistances: resistance due to the stationary liquid membrane, the boundary layer resistance in the sweep water side and the gas film resistance from the pore which separates the two liquids. However, for the gas system and the sweep liquid being studied in the present work, the gas phase resistance in the pores is negligible compared to the other two liquid phase resistances. The mass transfer coefficient for each gas species corresponding to the sweep water flow 


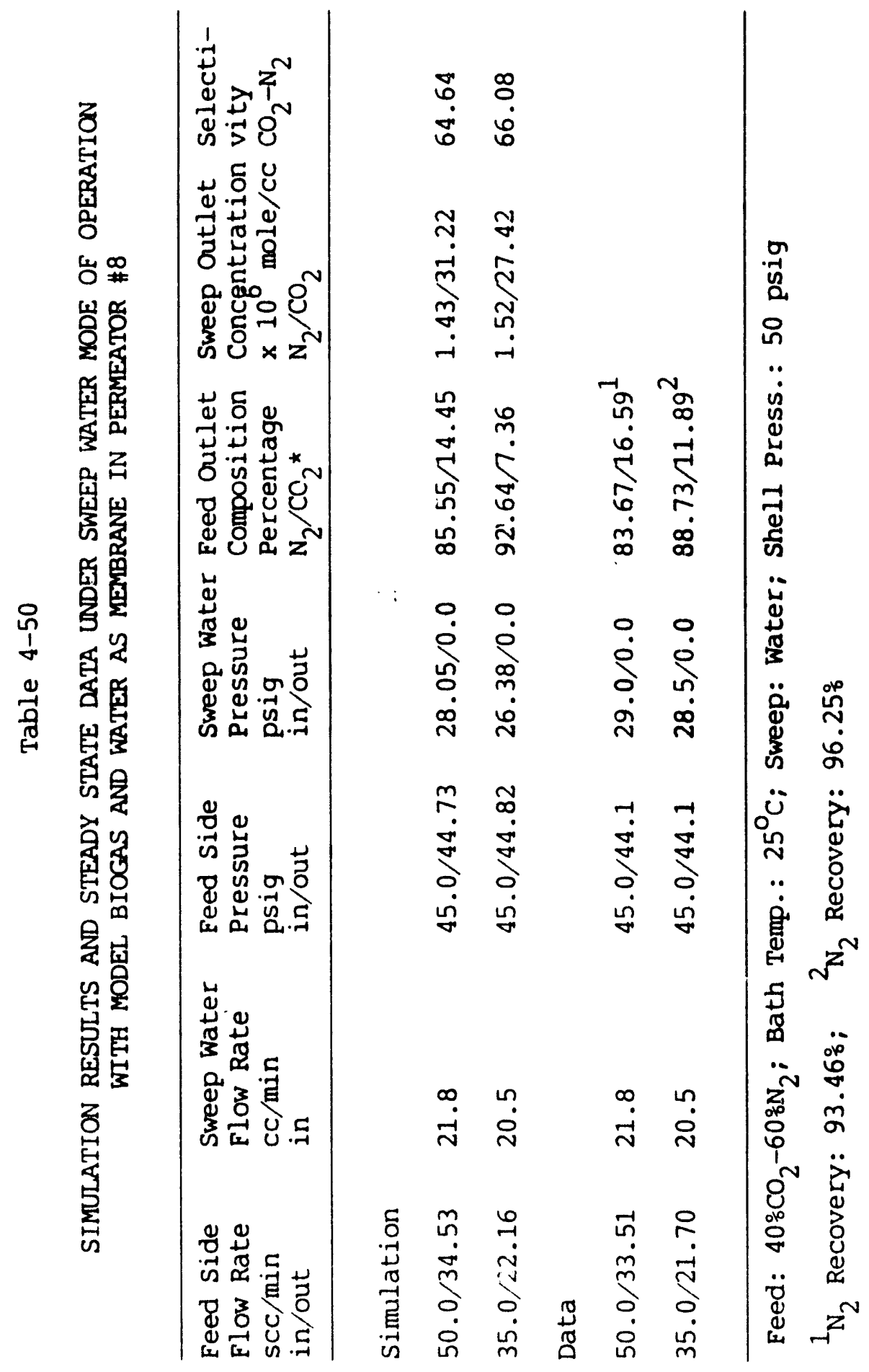




\section{FEED FIBER}

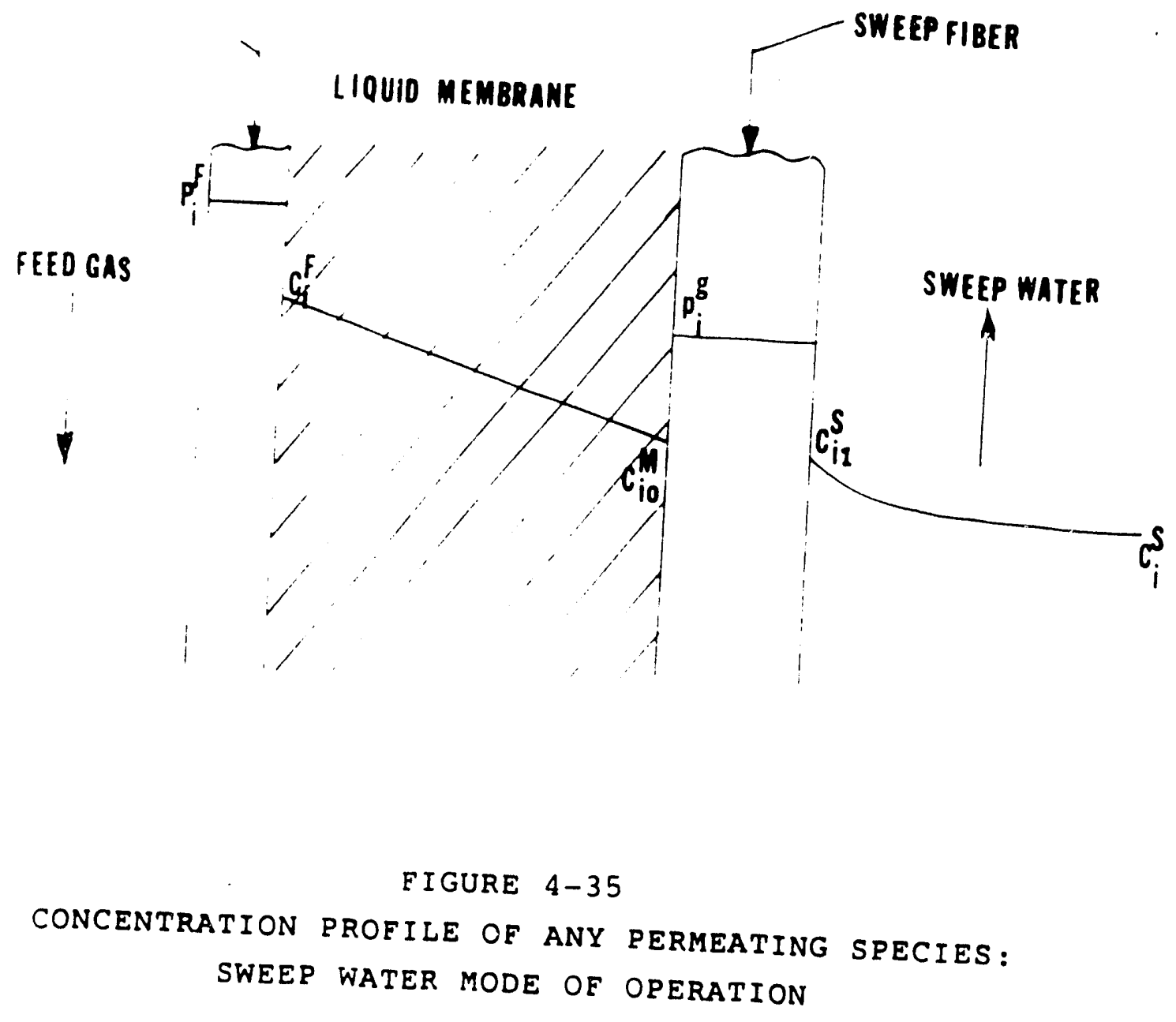


rate was calculated based on arithmetic concentration difference. The values are presented in Tables $4-51$ and $4-52$, respectively for permeators \#6 and \#8.

The overall selectivity was calculated in the following way. Total permeation rate of a species can be written as

$$
\begin{aligned}
R_{T i} & =\left(K_{O L}\right)_{i} 0^{\int^{l} t}\left(\pi D_{F O} N_{F} S_{i} P_{i}^{F}-\pi D_{S I} N_{S} C_{i}^{S}\right) d l \\
& =\left(K_{O L}\right)_{i} A_{F}\left[\Delta C_{a v g}\right]_{i}
\end{aligned}
$$

where $\left.\left[\Delta C_{a v g}\right]_{i}=l{ }_{0}^{1} t\left(\pi D_{F O} N_{F} S_{i} P_{i}^{F}-\pi D_{S I} N_{S} C_{i}^{S}\right) d l\right] / A_{F}$

and

$$
A_{F}=\pi D_{F O} N_{F} I_{t}
$$

The selectivity between species $i$ and $j$ is

$$
\alpha_{i j}=\frac{\left[R_{T} / \Delta C_{\text {avg }}\right\rfloor_{i} S_{i}}{\left[R_{T} / \Delta C_{a v g}\right\rfloor_{j} S_{j}}
$$

The $k_{m}$ values for $\mathrm{N}_{2}$ were higher than those for $\mathrm{CO}_{2}$ in all the cases. However, due to lower solubility of $\mathrm{N}_{2}$ in water, the overall selectivity is higher in the sweep water mode compared to sweep gas mode when water is used as a contained liquid membrane in both modes of operation. Note that the additional resistance in the sweep water side lowers the permeability of both the permeating species.

Water as Liquid Membrane and $40 \% \mathrm{CO}_{2}-60 \% \mathrm{CH}_{4}$ as Peed Gas

A purification run was carried out for an extended period of time using permeators \#6 and \#7 connected in series. Fresh sweep water was introduced separately into permeators \#6 and \#7. A regulating valve was provided at the uutlet end of one permeator to maintain equal flow rate of water through each permeator. The total sweep water flow rate was $250 \mathrm{cc} / \mathrm{min}$. The experimental results are presented in Table 4-53 and plotted in Figure 4-36. The methane recovery was greater than $95 \%$. However, the $\mathrm{CO}_{2}$ composition in the purified gas 


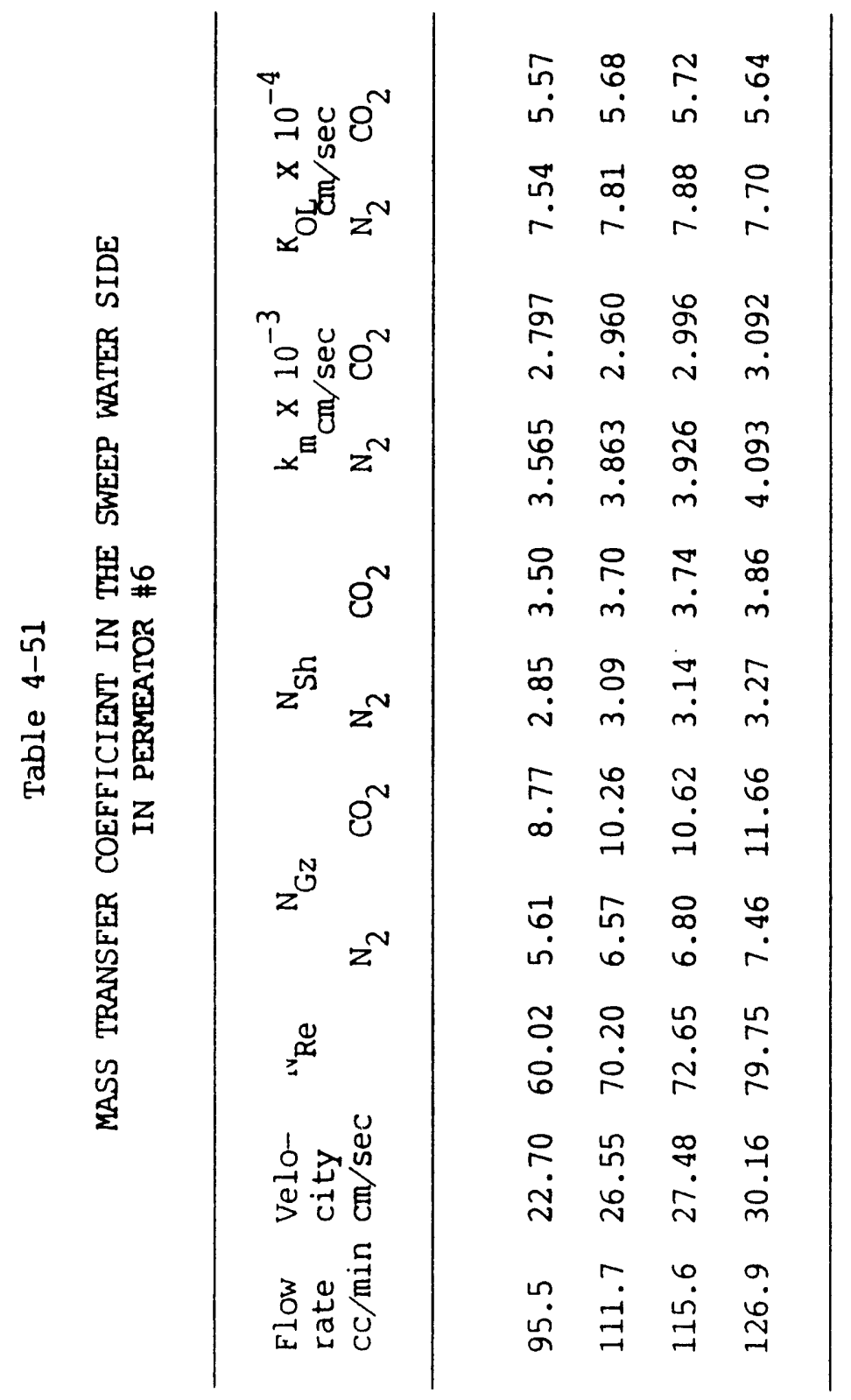




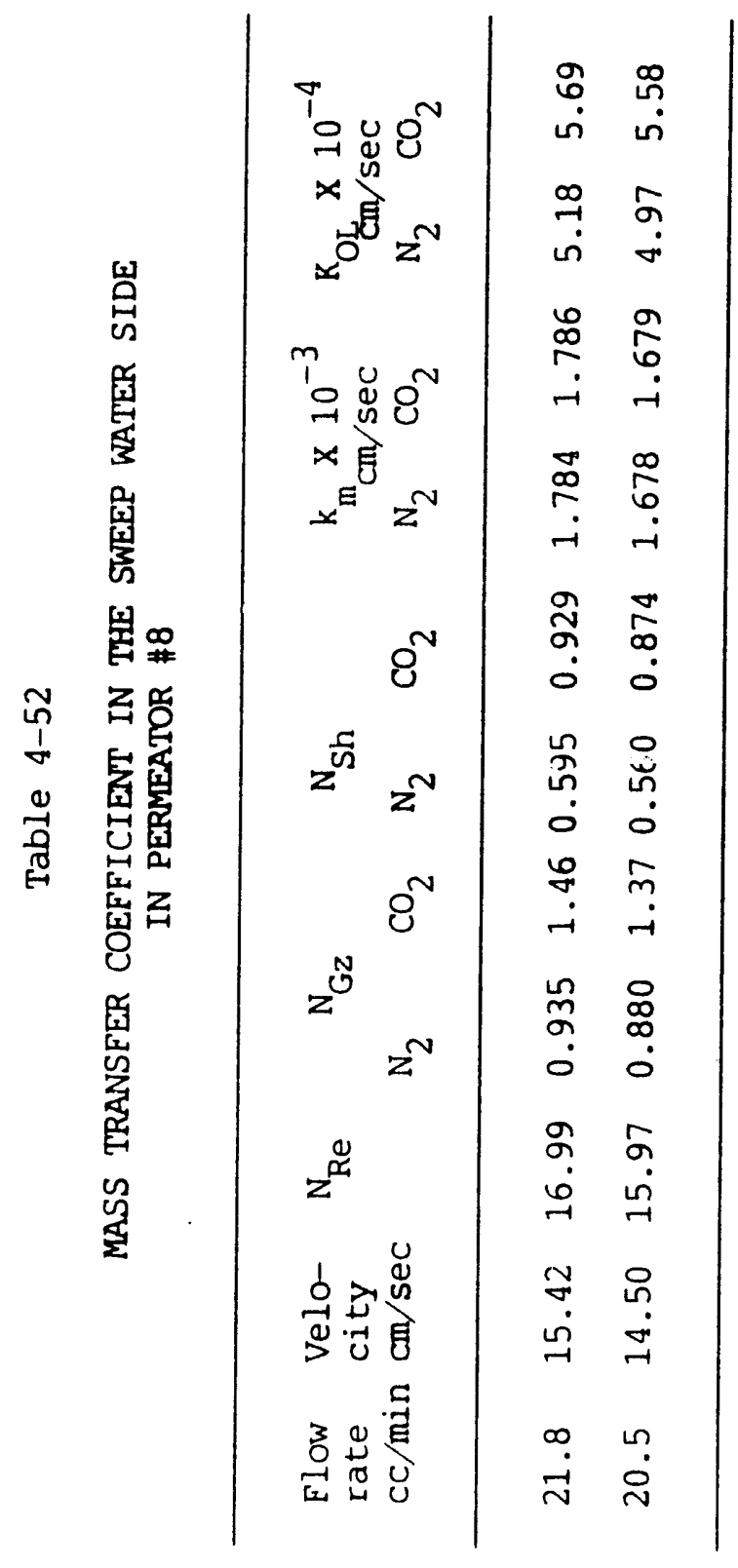




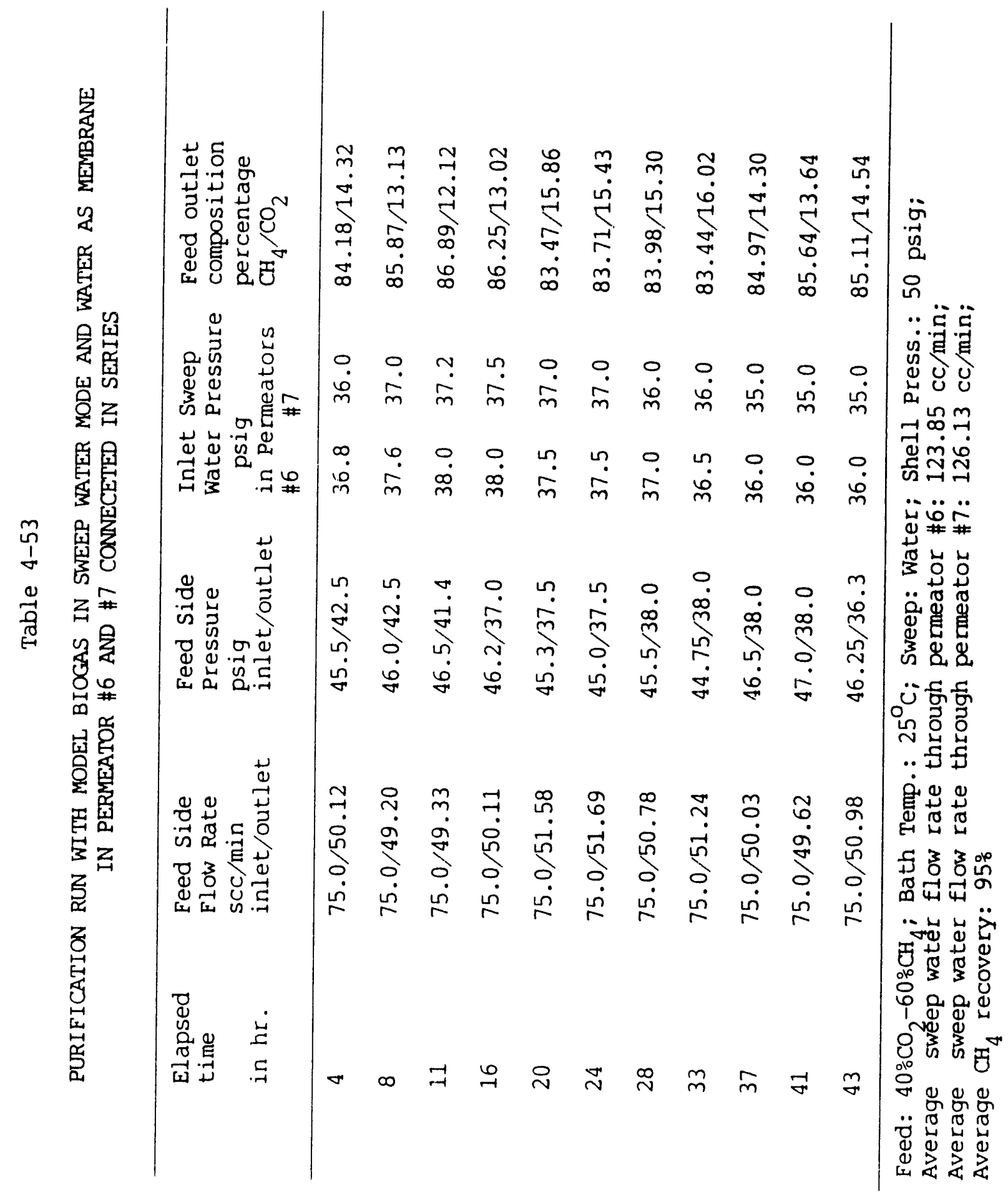




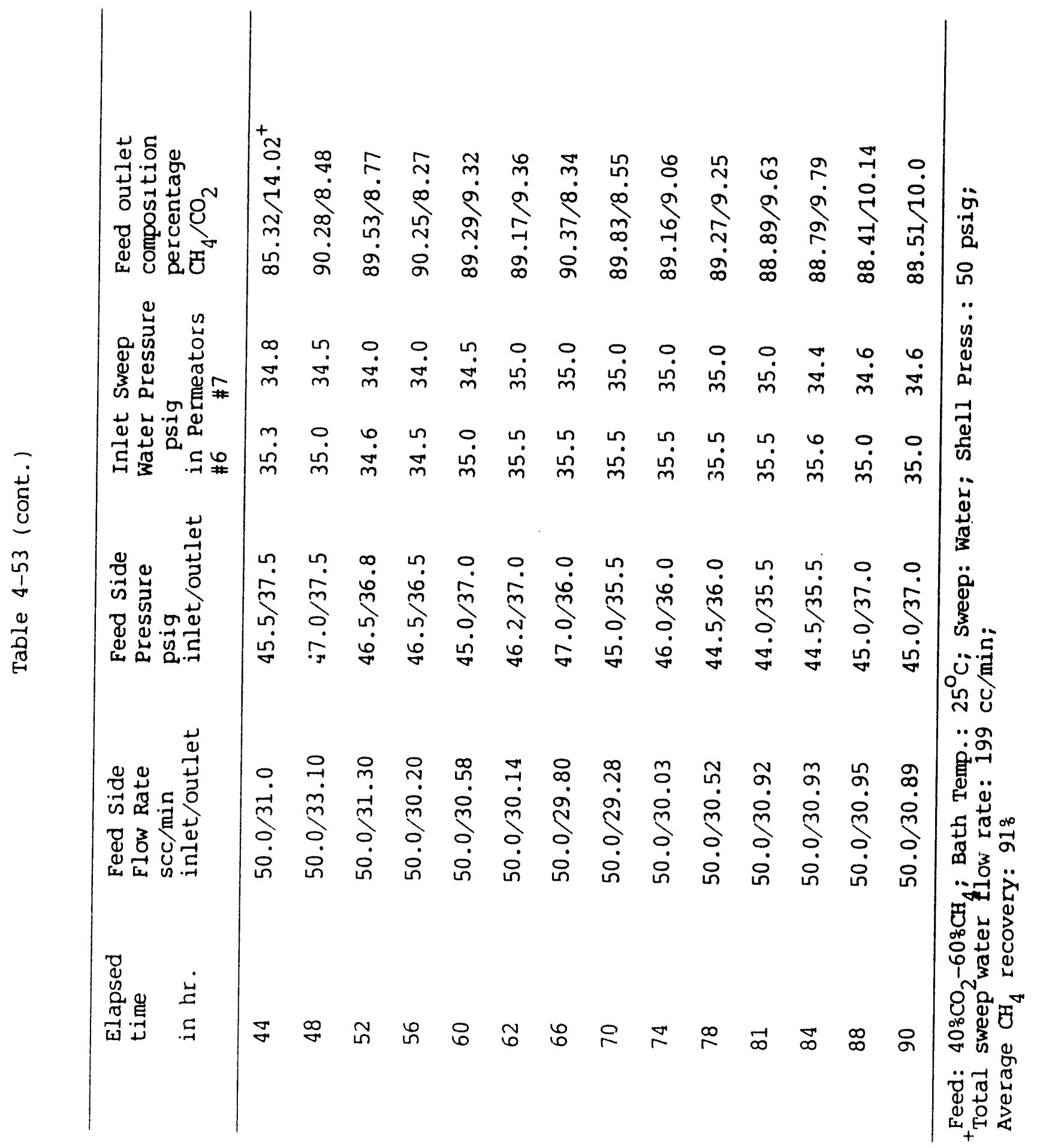




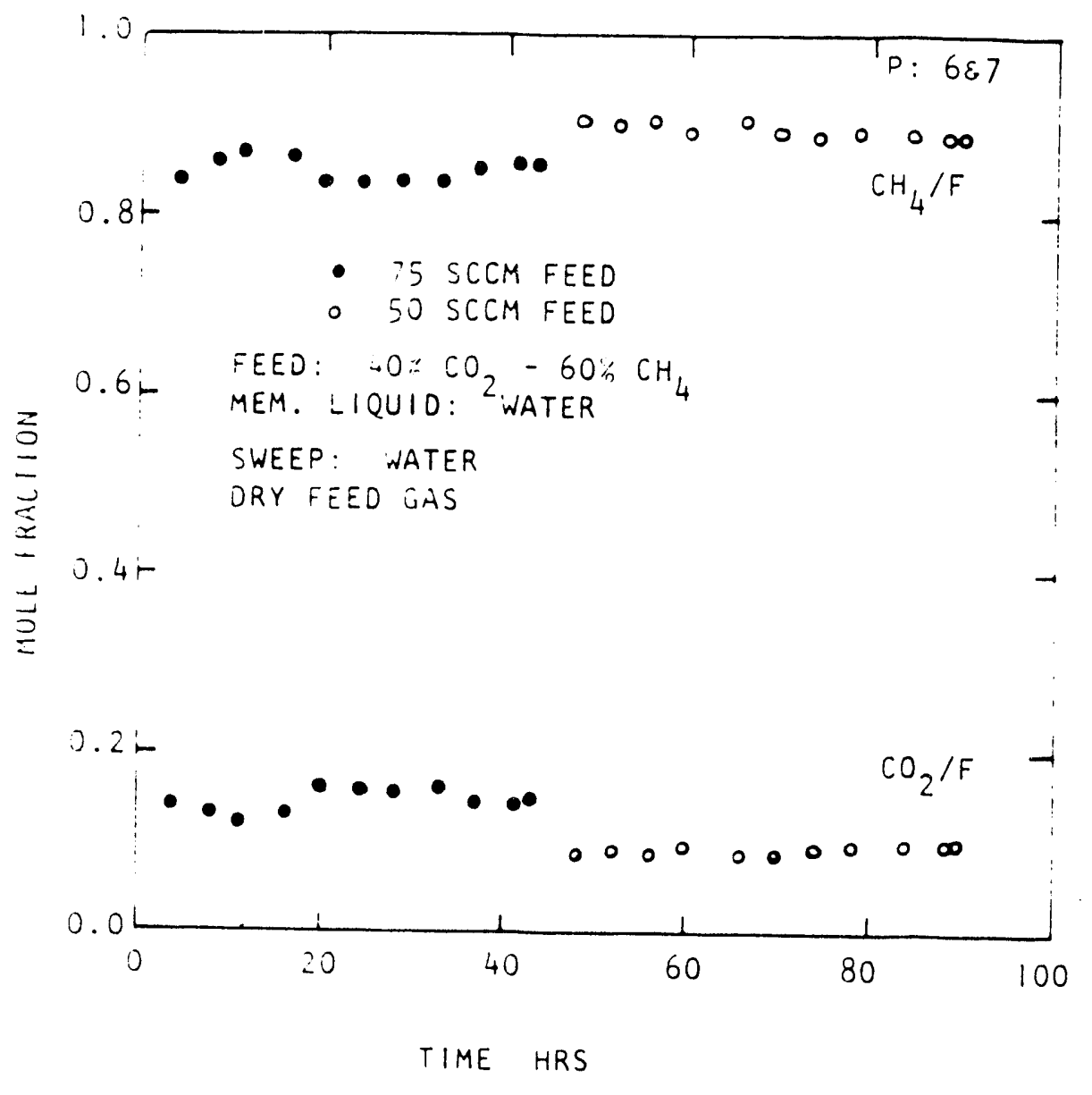

FIGURE $4-36$

CLM STABILITY STUDIES: SWEEP WATER MODE OF OPERATION WITH WATER AS MEMBRANE USING PERMEATORS \#6 AND $\# 7$ CONNECTED IN SERIES 
stream was relatively high.

After 43 hours of steady run, the feed gas flow rate was reduced, keeping the feed gas inlet pressure constant. This led to a lower $\mathrm{CO}_{2}$ composition at the feed gas outlet (about $10 \%$ ). The average methane recovery was $91 \%$.

30 wtz $\mathrm{R}_{2} \mathrm{CO}_{3}$ Solution as Liquid Membrane and $40 \% \mathrm{CO}_{2}-60 \% \mathrm{~N}_{2}$ as Peed Gas

Model biogas purification run with sweep water and $30 \mathrm{wt} \% \mathrm{~K}_{2} \mathrm{CO}_{3}$ solution as membrane was carried out in permeator \#6. This run was continued for about 50 hours. The results are shown in Table 4-54. The major difference from all the experiments carried out so far with $30 \mathrm{wt} \% \mathrm{k}_{2} \mathrm{CO}_{3}$ solution was that the feed gas in this case was completely dry. The run was carried out without any stability problem. However, the quality of the purified gas is lower in the present case compared to pure water membrane experiments which can be attributed to the lower permeability of gases through the $30 \mathrm{wt} \% \mathrm{~K}_{2} \mathrm{CO}_{3}$ solution and to a much lower water flow rate. Average nitrogen recovery was about $95 \%$.

30 wtX $\mathrm{K}_{2} \mathrm{CO}_{3}$ Solution as Liquid Membrane and $40 \pi \mathrm{CO}_{2}-60 x \mathrm{CB}_{4}$ as Peed Gas

In this study, permeators \#6 and \#7 were operated in series. Sweep water was introduced separately through permeators \#6 and \#7. As in the previous case, the feed gas was introduced without any humidification.

The results are presented in Table 4-55 and plotted in Figure 4-37. The $\mathrm{CO}_{2}$ composition in the purified feed gas varied between 14 to $16 \%$. The methane recovery was around $93 \%$. The above set of experiments strongly suggest that a trouble-free operation with sweep water mode of operation is obviously feasible. Further high methane recovery is easily achieved.

20 wtz DEA Solution as Liquid Membrane and $40 \% \mathrm{Co}_{2}-60 \% \mathrm{~N}_{2}$ as Peed Gas

A long-term experiment using 20 wt\% DEA solution as a contained liquid membrane was carried out in permeator \#8. Experimental data are reported in Table 4-56. The operation was very stable even though the feed gas mixture was totally dry. For a feed gas flow rate of $50 \mathrm{sccm}$ and sweep water flow rate of $25 \mathrm{cc} / \mathrm{min}$, 


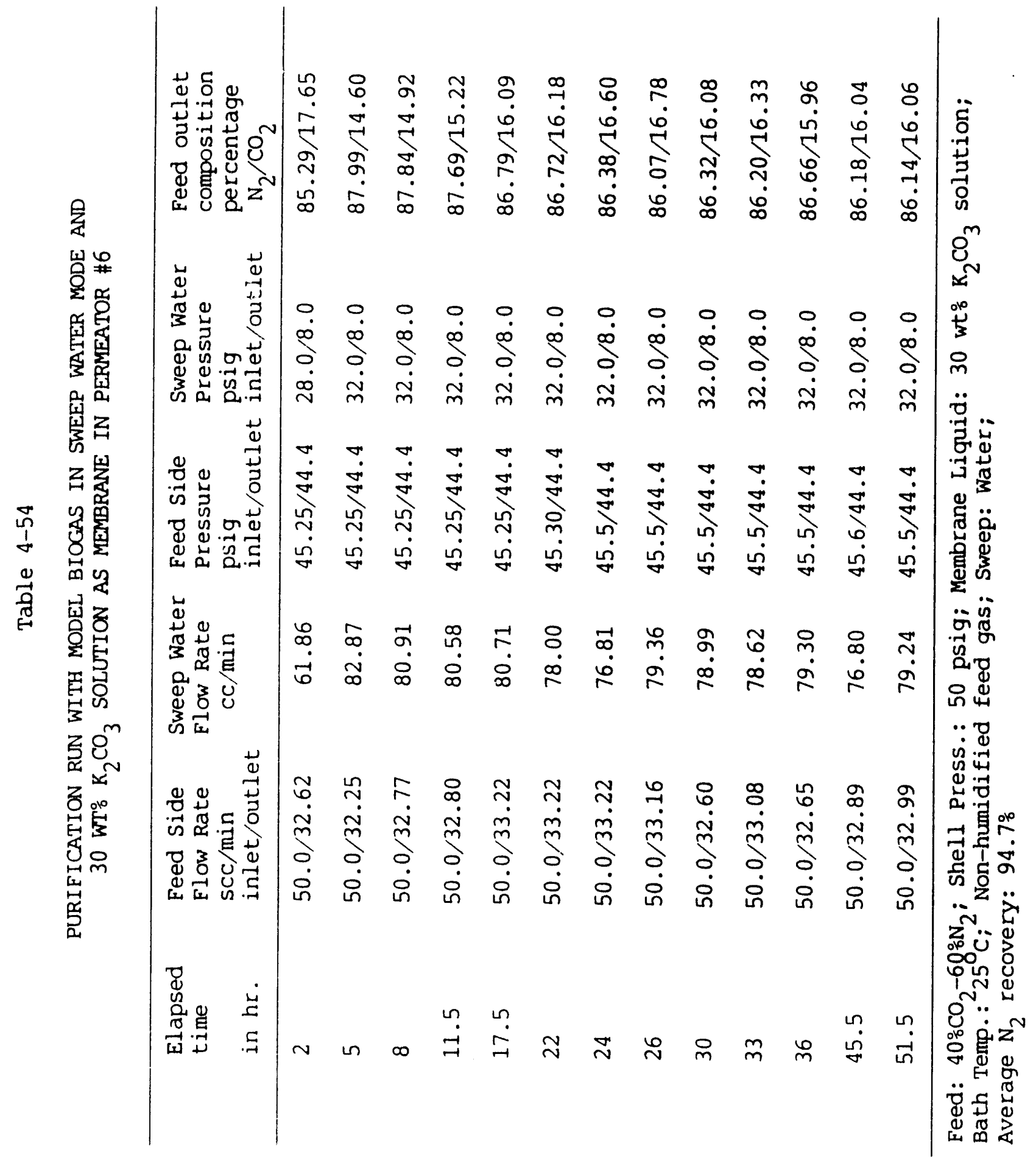




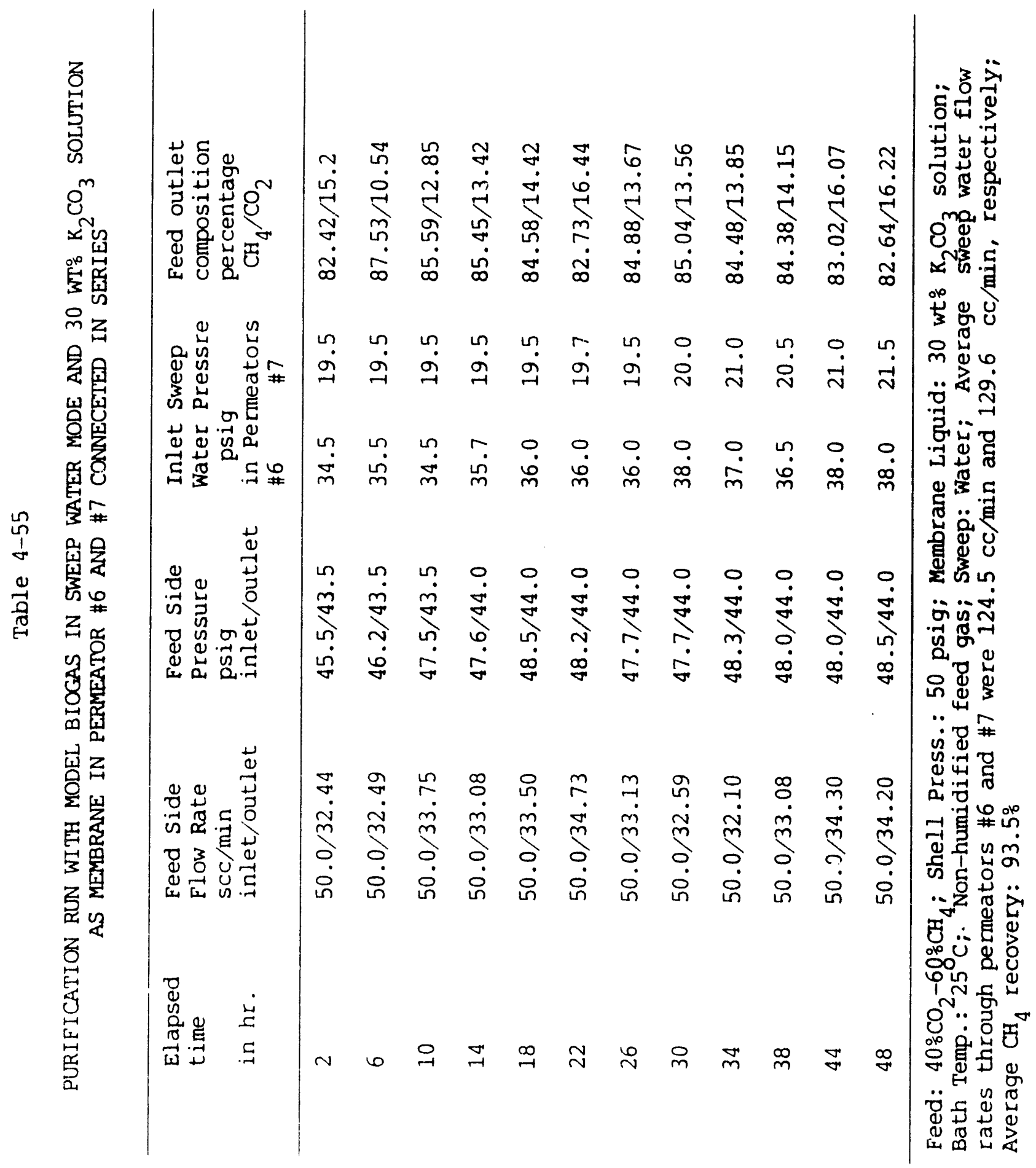




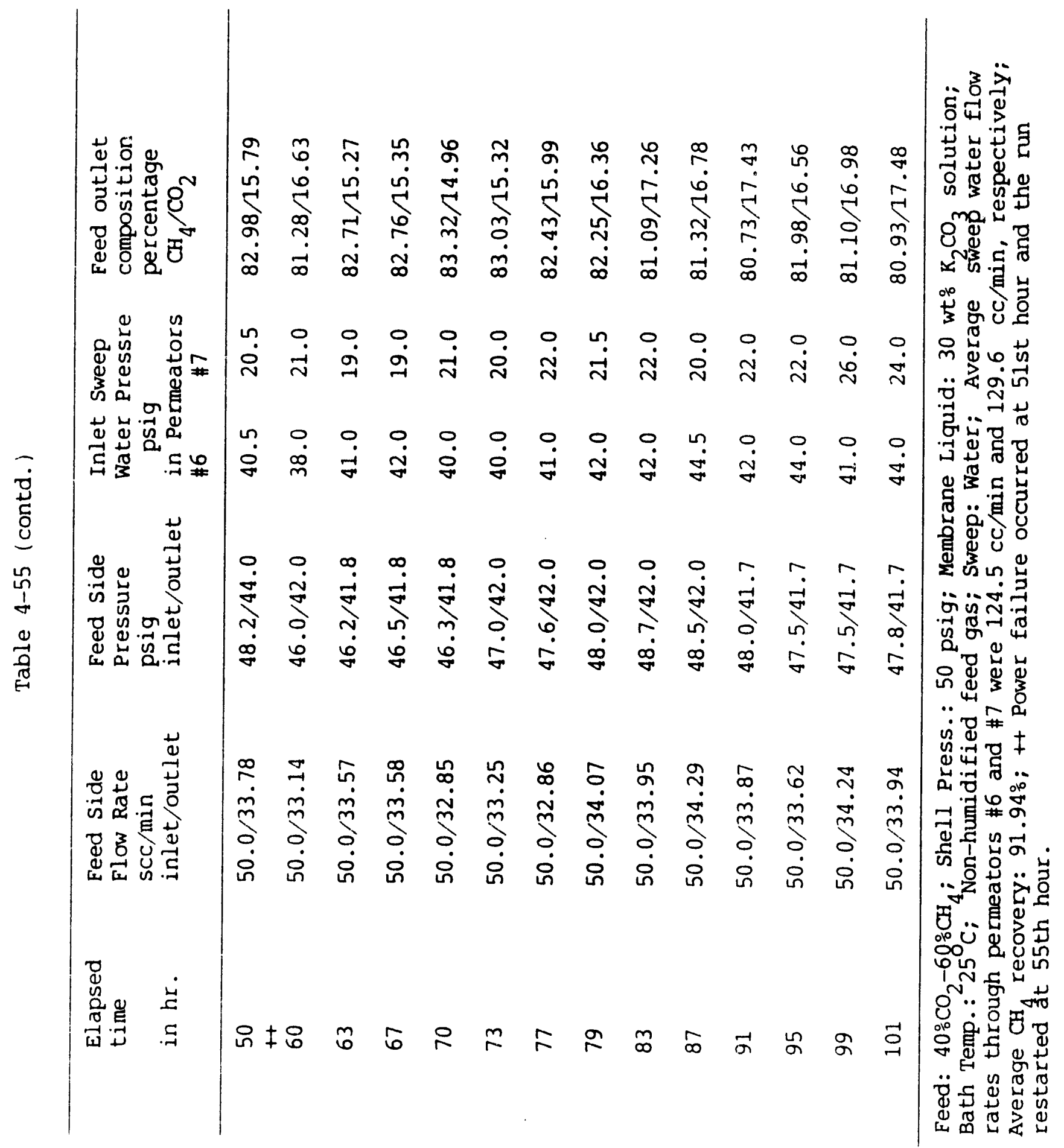




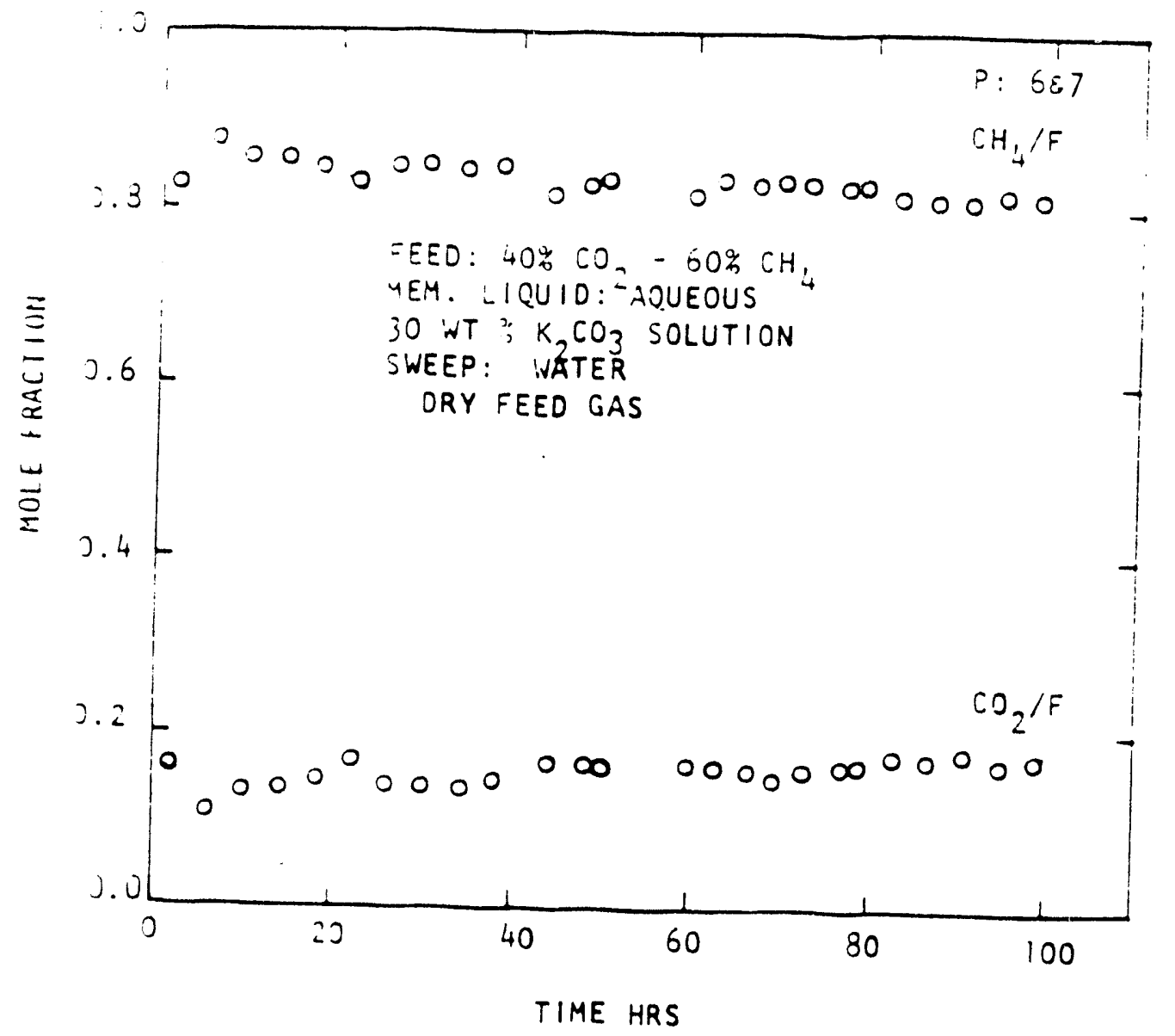

FIGURE $4-37$

CLM STABILITY STUDIES: SWEEP WATER MODE OF OPERATION WITH 30 WT $\mathrm{K}_{2} \mathrm{CO}_{3}$ SOLUTION AS MEMBRANE USING PERMEATORS \#6 AND \# 7 CONNECTED IN SERIES 


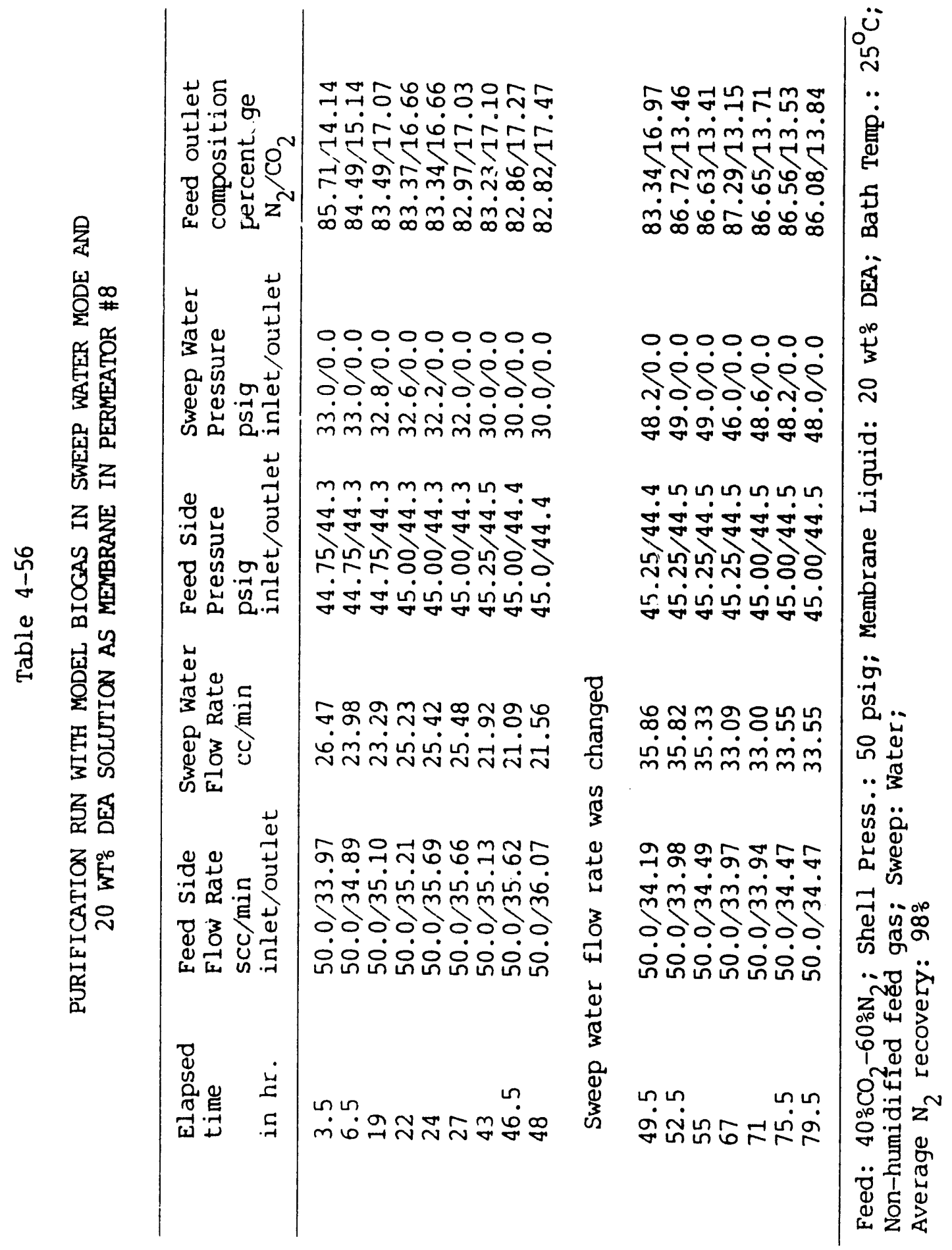




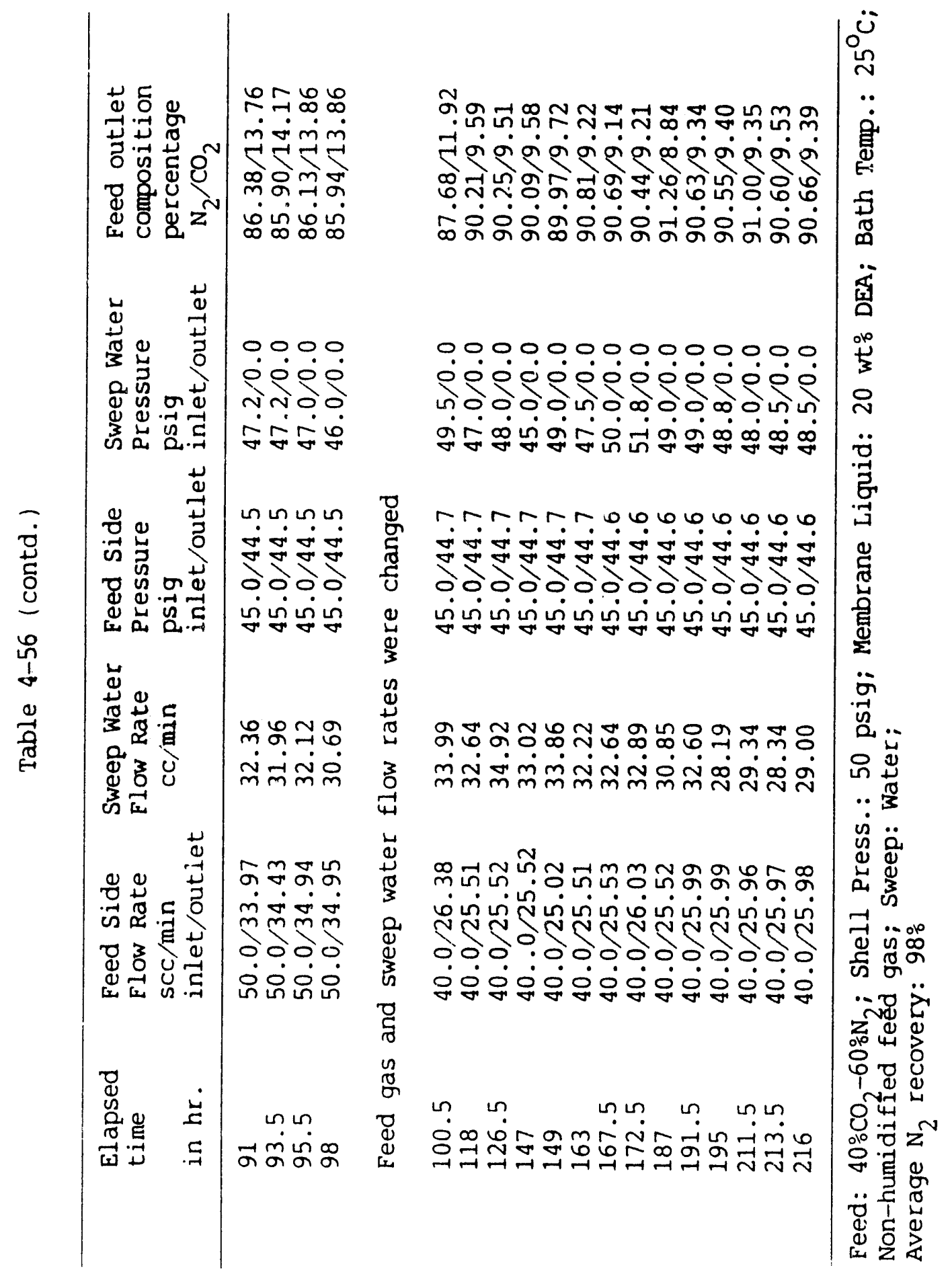


feed oulet composition stabilized at around $17 \% \quad \mathrm{CO}_{2}$. After 48 hours of operation, the sweep water flow rate was increased. A better performance was obtained at higher water flow rate. The $\mathrm{CO}_{2}$ composition dropped to around $13-14 \%$. After another 50 hours of stable operation, the feed flow rate was lowered to a value of $40 \mathrm{sccm}$. The $\mathrm{CO}_{2}$ composition in the feed outlet dropped to around $9 \%$. The performance was remarkably stable throughout the rest of the experiment. Nitrogen recovery was around $98 \%$. This shows the robust and trouble-free operation with DEA as liquid mebrane in sweep water mode is possible with high recovery and reasonably high purity with only a 17 -inch-long permeator. Note that the sweep water flow rates used were $1 / 2$ to $1 / 3$ of the sweep water flow rates used with permeators \#6 and \#7.

\section{ESTIMATION OP EFFECTIVE MEMBRANE THICRNESS IN LARGE PERMEATORS}

The experimental procedure for determination of the effective membrane thickness in a large permeator was similar to that for a small permeator. Pure gas permeation studies under conventional mode of operation with water as a liquid membrane have been carried out in all large permeators. The permeated gas was allowed to flow countercurrent to the feed gas stream and it exited the permeator essentiaily at atmospheric pressure. The permeate flow rate was measured accurately by a bubble flow meter. The governing differential equations for pure gas permeation were then solved for different values of effective membrane thickness. The correct value should match the gas permeation rate obtained experimentally. The pure $\mathrm{CO}_{2}$ permeation data for large permeators $\# 10$, \#11 and \#12 at different feed flow rates are compared with the simulation results in Tables 4-57, 4-58 and 4-59, and the effective membrane thicknesses are estimated to be in the range of 0.085 irch, 0.0125 inch and 0.011 inch, respectively.

Permeator \#10 was the first large-scale permeator fabricated. A totally new fiber matting and bundle preparation procedure was adopted as the number of fibers were very large. In this permeator the feed and the sweep fibers did not mix properly during the bundle preparation procedure as the cotton threads were inserted in between the two fiber layers after the mat was prepared (see Section 3). This caused the two layers of fibers to stay more or less as two separate bunches which resulted in a higher membrane thickness. However, this 


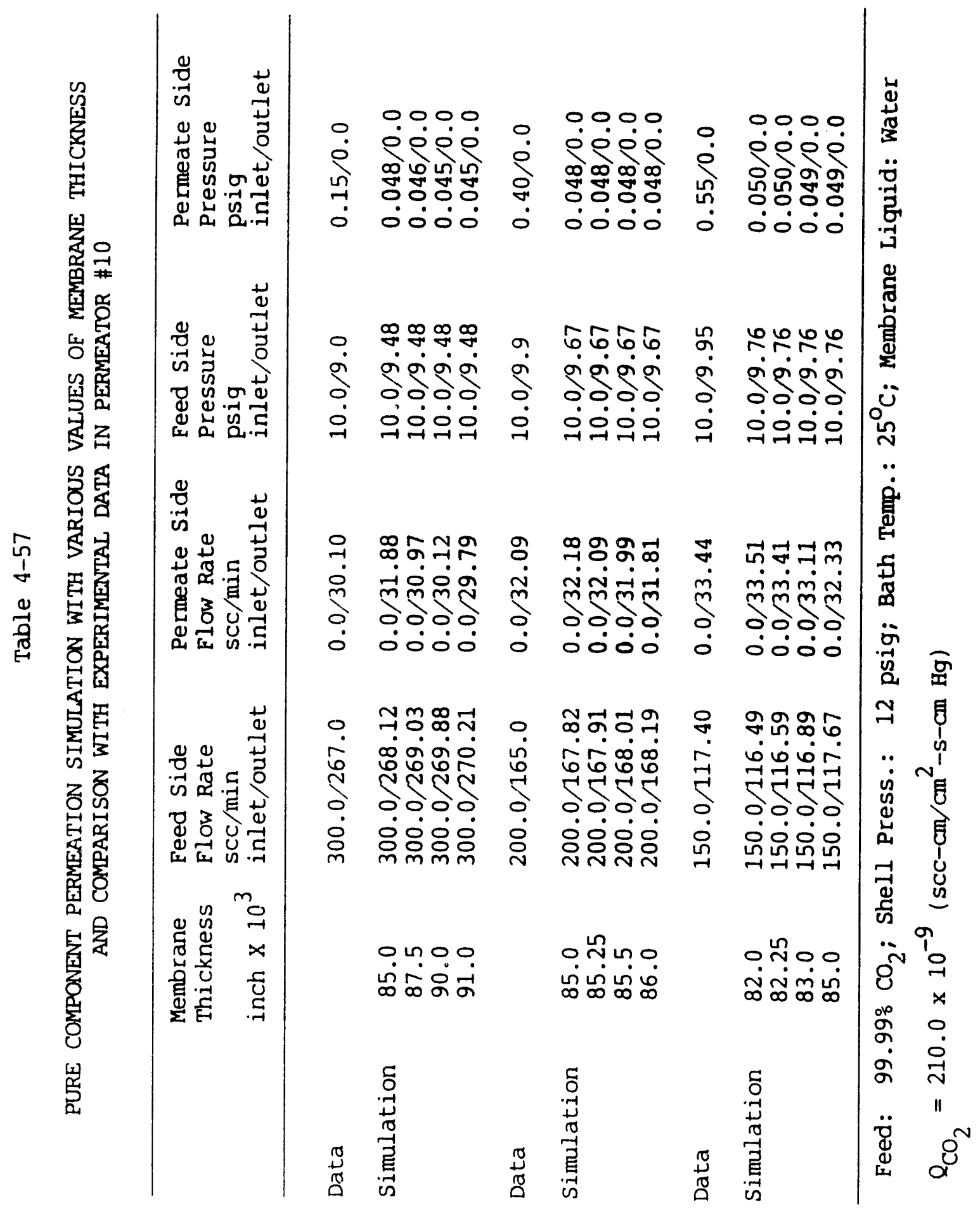




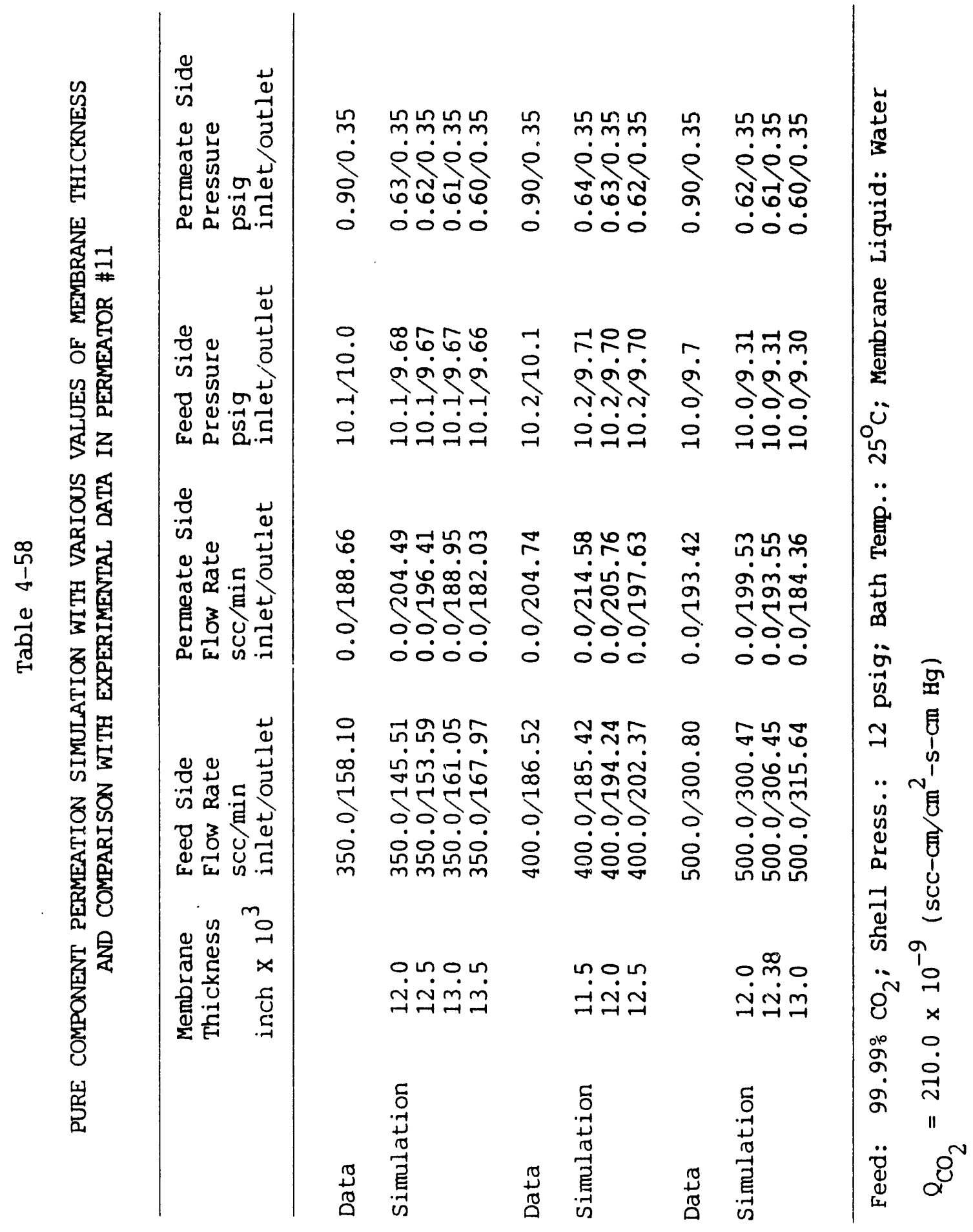




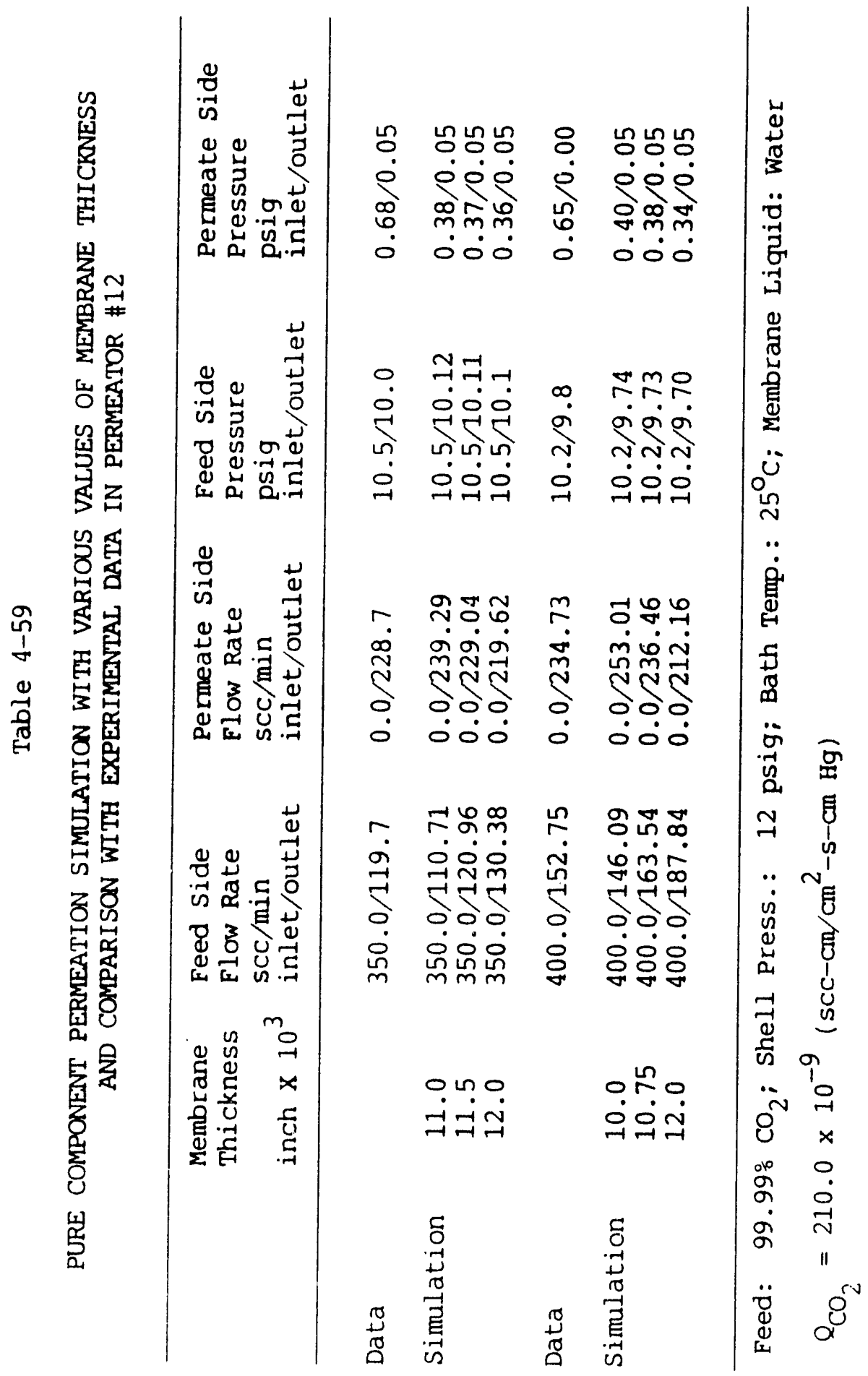


problem was eliminated in the making of the large permeators \#11 and \#12.

A similar gas permeation experiment was also carried out in Permeator \#11 with $\mathrm{N}_{2}$ as a feed gas. The steady state experimental data and the simulation results are shown in Table 4-60. This experiment indicated a liquid membrane thickness of 0.006 inch which was much lower than that obtained with pure $\mathrm{CO}_{2}$ permeation studies. Steady state separation runs with $\mathrm{CO}_{2}-\mathrm{N}_{2}$ gas mixture, described later, were carried out in order to explain this anomalous behavior.

\section{PURIFICATION STUDIES OF MODEL BIOGAS MIXTURES IN LARGE SCALE OPERATION}

Model biogas purification studies in large-scale operation were carried out with Permeators \#11 and \#12 only under sweep gas mode of operation. Pure water and aqueous solution of $20 \mathrm{wt} \%$ DEA were utilized as liquid membranes. For pure water membrane, the gas streams were not humidified. For long-term experiment, the feed gas mixture was created by blending commercial grade $\mathrm{CO}_{2}$ and $\mathrm{N}_{2}$ (or $\mathrm{CH}_{4}$ ) gases. The aciual gas composition was determined at the end of the experiment by injecting the feed mixture into the gas chromatograph column. All experiments were carried out at $25^{\circ} \mathrm{C}$. Pure helium gas was used to pressurize the shell liquid storage tank. For $\mathrm{CO}_{2}-\mathrm{N}_{2}$ feed gas mixture, helium was used as a sweep gas whereas $\mathrm{N}_{2}$ sweep gas was used for $\mathrm{CO}_{2}-\mathrm{CH}_{4}$ feed gas mixture.

Water as Liquid Membrane and $\mathrm{CO}_{2}-\mathrm{N}_{2}$ Gas Mixture as Feed

Two different values of membrane thickness were obtained for permeator \#11. These two different values were determined from pure $\mathrm{CO}_{2}$ and pure $\mathrm{N}_{2}$ permeation studies through pure water as a liquid membrane. A hollow-fiber-contained liquid membrane permeator has four potted ends. Any one of them can be used to introduce the feed gas. For pure $\mathrm{N}_{2}$ permeation run, the feed nitrogen was introduced through the potted end \#2. The same end was not utilized for pure $\mathrm{CO}_{2}$ permeation studies. It was anticipated that there was some contamination of the feed gas into the sweep gas when a particular potted end was used as the feed inlet end. In order to resolve the problem and to identify the potted end, four steady state purification runs were carried out under identical conditions. In each case, the feed inlet end was changed. The feed and sweep gas flow rates were maintained at 750 and $1000 \mathrm{sccm}$, respectively. In all runs 


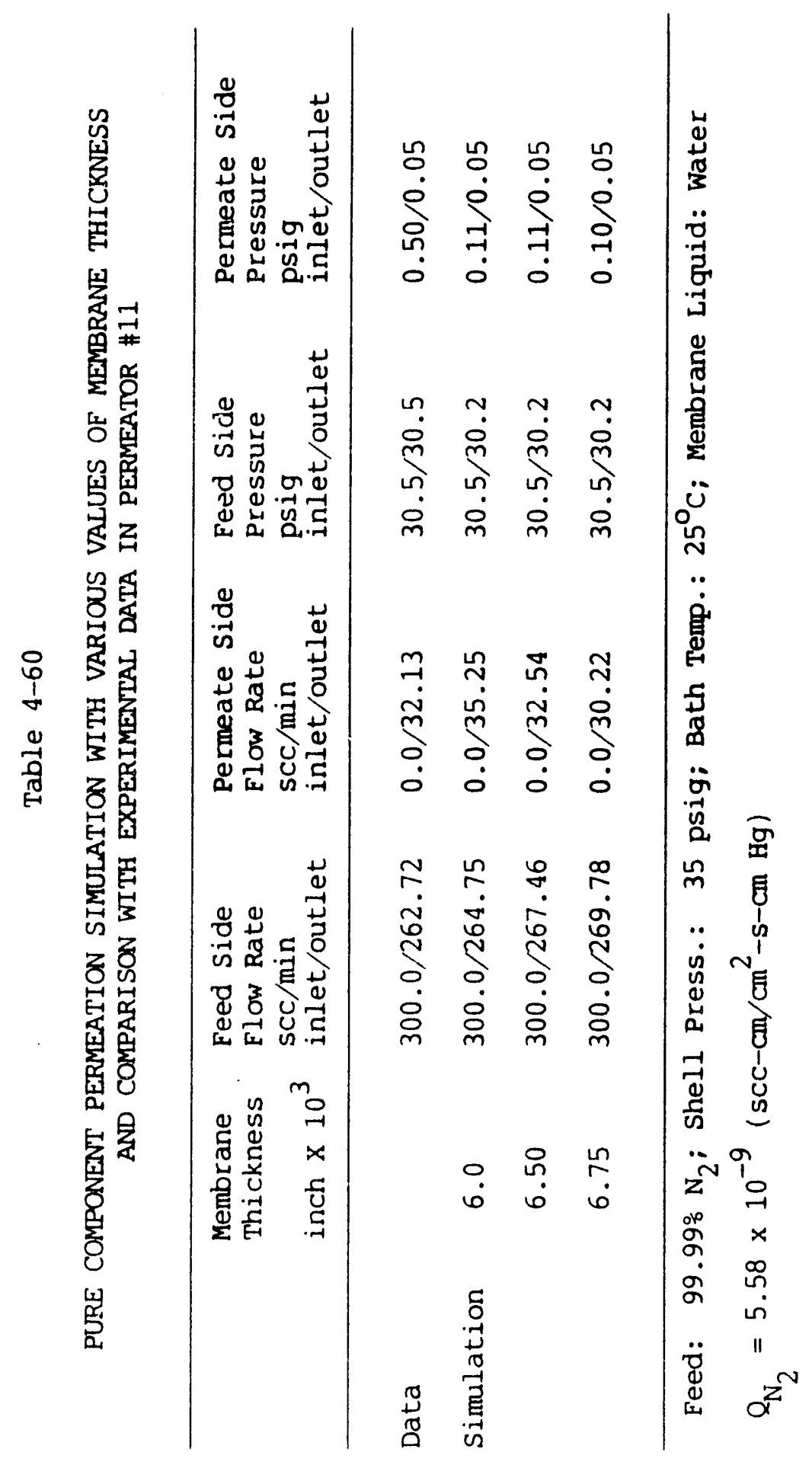


dry feed gas was introduced into the permeator at an inlet pressure of 45 psig. The sweep helium gas was introduced countercurrent to the feed gas mixture and the liquid membrane pressure was kept at 50 psig.

The steady state results of the separation runs are presented in Table 4-61. The permeation rates in the first two cases are considerably higher than the other two suggesting some kind of contamination. The nitrogen recovery value is also considerably low. Note that, for the first two data sets the feed gas was introduced through the bent fiber set. It was clear that high-pressure feed gas could not be used in the bent fiber set. In subsequent experiments, the feed was therefore introduced through the straight fiber set.

To determine the stability of a large-scale hollow-fiber-contained liquid membrane system, separation studies were carried out over a period of 245 hrs. ( 10 days) using $41 \% \mathrm{CO}_{2}-58 \% \mathrm{~N}_{2}$ ( $1 \%$ impurities) model biogas mixture as feed in permeator \#11. As Table 4-62 indicates, the flow rates and pressures of various streams were stable. The performance was essentially steady with an average $\mathrm{N}_{2}$ recovery of $-87 \%$. The $\mathrm{CO}_{2}$ composition in the purified gas stream was in the range of 12 to $14 \%$. The pressure drop in the feed stream was negligible and that of the sweep stream was only about 5 psi for the flow rates used in this study. The compositions of the feed and sweep outlet streams for the entire period are shown in Figure 4-38.

The results demonstrate that long-term separation of $\mathrm{CO}_{2}$ can be carried out efficiently and stably in a large-scale hollow-fiber-contained liquid membrane permeator with pure water as a liquid membrane without any operational difficulty.

20 t $\%$ DEA Solution as Liquid Membrane and $\mathrm{CO}_{2}-\mathrm{N}_{2}$ Gas Mixture as Peed

One month-long life test of a hollow-fiber-contained liquid membrane permeator system was made with a feed mixture containing $41 \% \mathrm{CO}_{2}$ and $58 \% \mathrm{~N}_{2}$. Two large permeators (\#11 and \#12) were connected in series for this study i.e., the purified gas stream from the first permeator was introduced as the feed in the second permeator. However, no such series connection was made in the sweep line. Pure helium sweep gas was introduced in each permeator. (The connections 


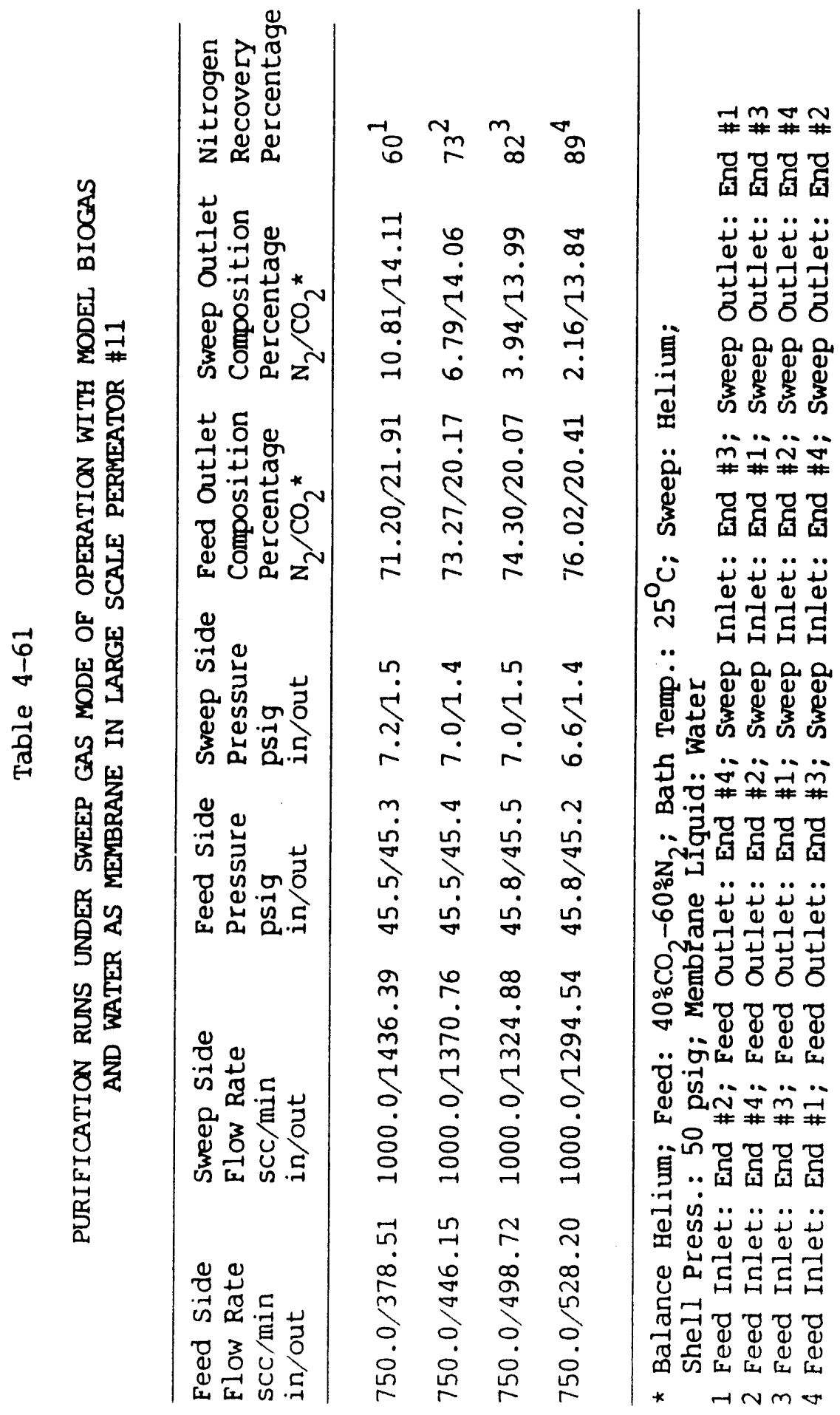




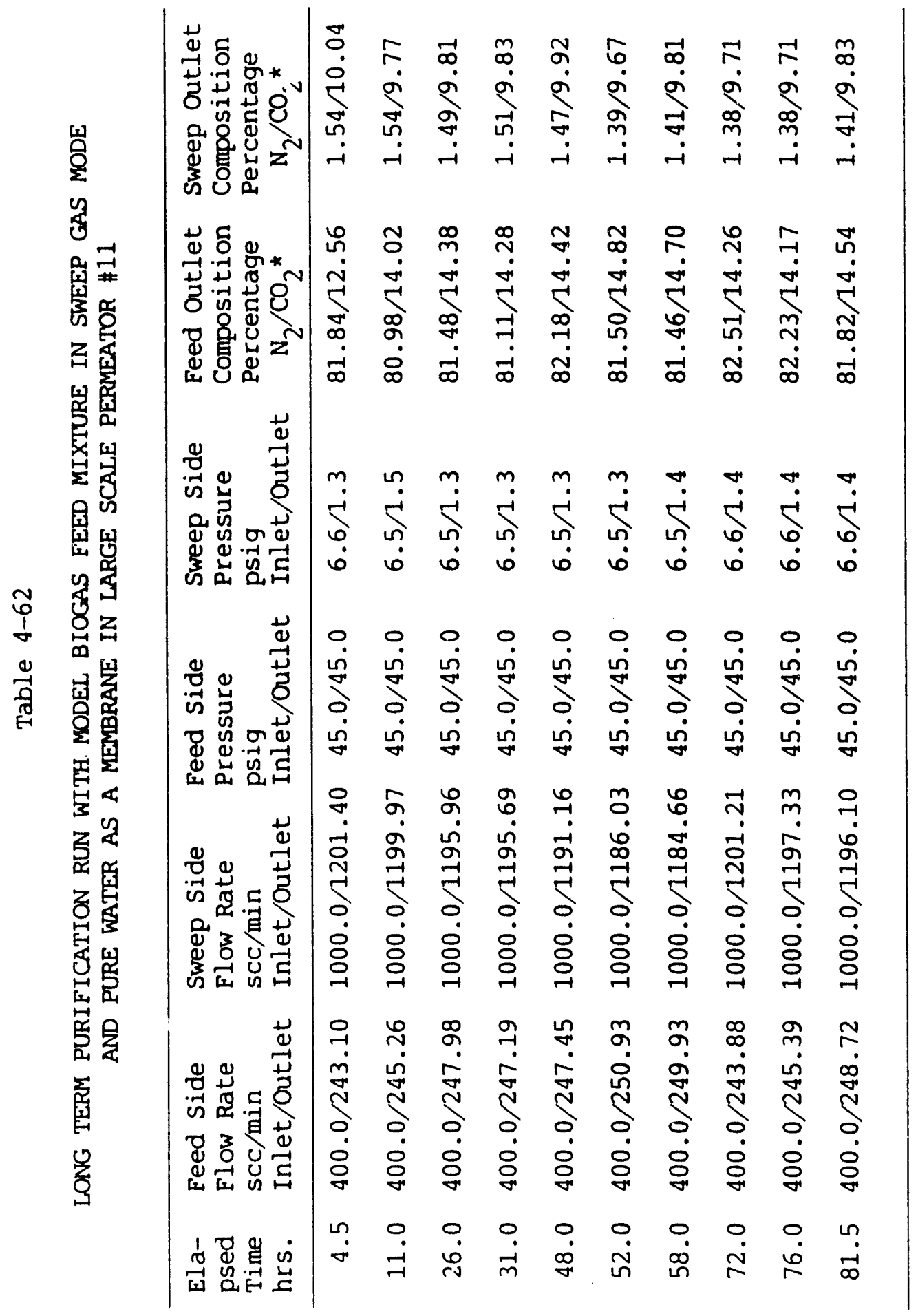




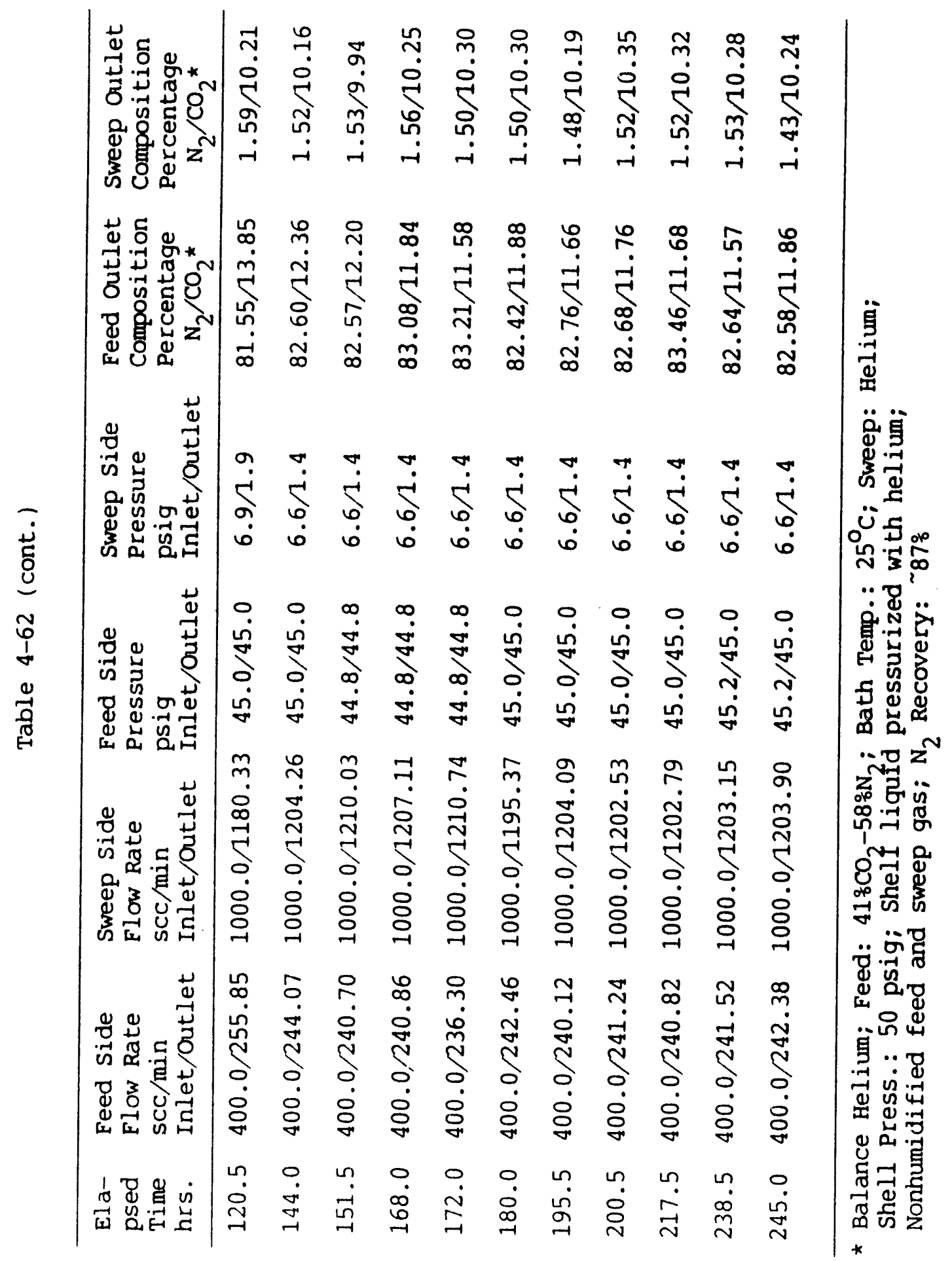




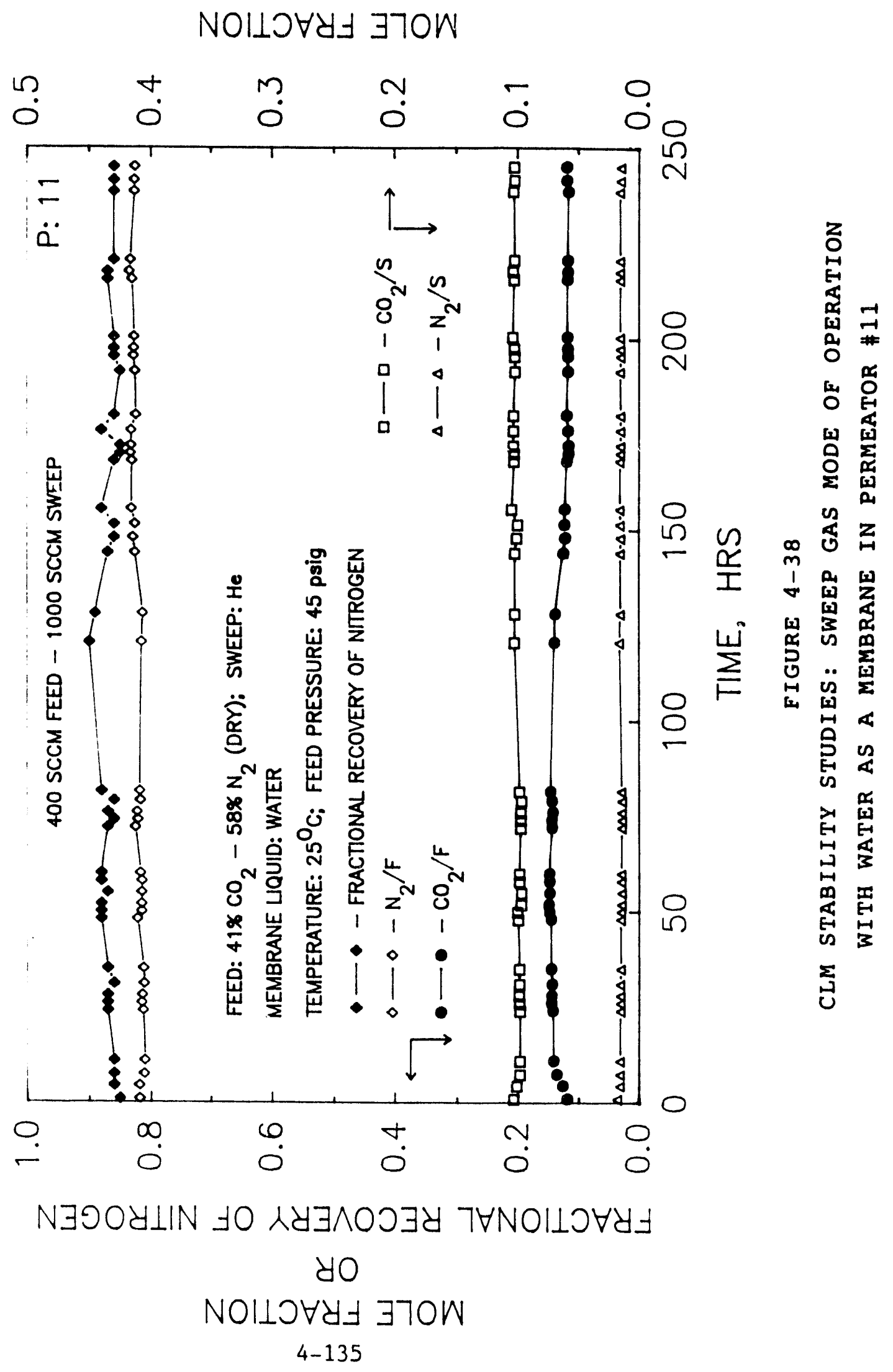


were made as follows. After measurement of the total sweep inlet flow rate, the stream was divided in two by a tee and was connected to the sweep inlet end of each permeator. Similarly, the sweep outlet streams from two permeators were also joined by another tee before it was sent to the GC for analysis.)

Initially, a feed flow rate of $350 \mathrm{scc} / \mathrm{min}$ was maintained at a pressure of 45 psig for about 2 days. The total sweep flow rate was $1200 \mathrm{scc} / \mathrm{min}$. There was virtually no pressure drop in the feed line and the purified gas contained only 3 to $4 \%$ of $\mathrm{CO}_{2}$. The average $\mathrm{N}_{2}$ recovery was $79 \%$. This observation led us to believe that the available membrane area in two permeators would be able to process a higher feed flow rate. Therefore, after two days of operation, the feed flow rate was increased to $475 \mathrm{scc} / \mathrm{min}$ without altering the total sweep flow rate. The performance for the entire period is shown in Table 4-63. The outlet gas compositions and the $\mathrm{N}_{2}$ recovery are plotted against the elapsed time in Figure 4-39. An excellent and steady performance was observed. At higher feed flow rate of $475 \mathrm{scc} / \mathrm{min}$, the $\mathrm{CO}_{2}$ content in the purified gas was generally less than $10 \%$ with an average $\mathrm{N}_{2}$ recovery of $87 \%$. The pressure drop in both streams was very low. To avoid local drying of the membrane liquid over such a long period of time, about $50 \mathrm{ml}$. of membrane liquid was purged out from each permeator after every 5 days of continuous operation without disturbing the experiment.

The results show that the hollow-fiber-contained liquid membrane system can be used efficiently to separate gas mixtures with minimum maintenance over a prolonged time period. In an actual operation a slow and steady recirculation of the membrane liquid (if the membrane liquid is a solution instead of a pure liquid) would completely eliminate the problem of local drying, if any, within the permeator.

THO more short-term experiments were carried out with $\mathrm{CO}_{2}-\mathrm{N}_{2}$ feed mixture and 20 w $\%$ DEA solution as a liquid membrane. Both experiments used Permeators \#11 and \#12 in series as mentioned earlier. These two experiments were made in Lesponse to the erquiries by two companies e.g., Permapure Products, Inc. and Ecolotrol (see section 6). The first experiment utilized a feed gas mixture containing $41 \% \quad \mathrm{CO}_{2}$ and $58 \% \quad \mathrm{~N}_{2}$ but the feed inlet pressure was close to atmospheric (problen posed by Ecolotrol). The steady state data is shown in 


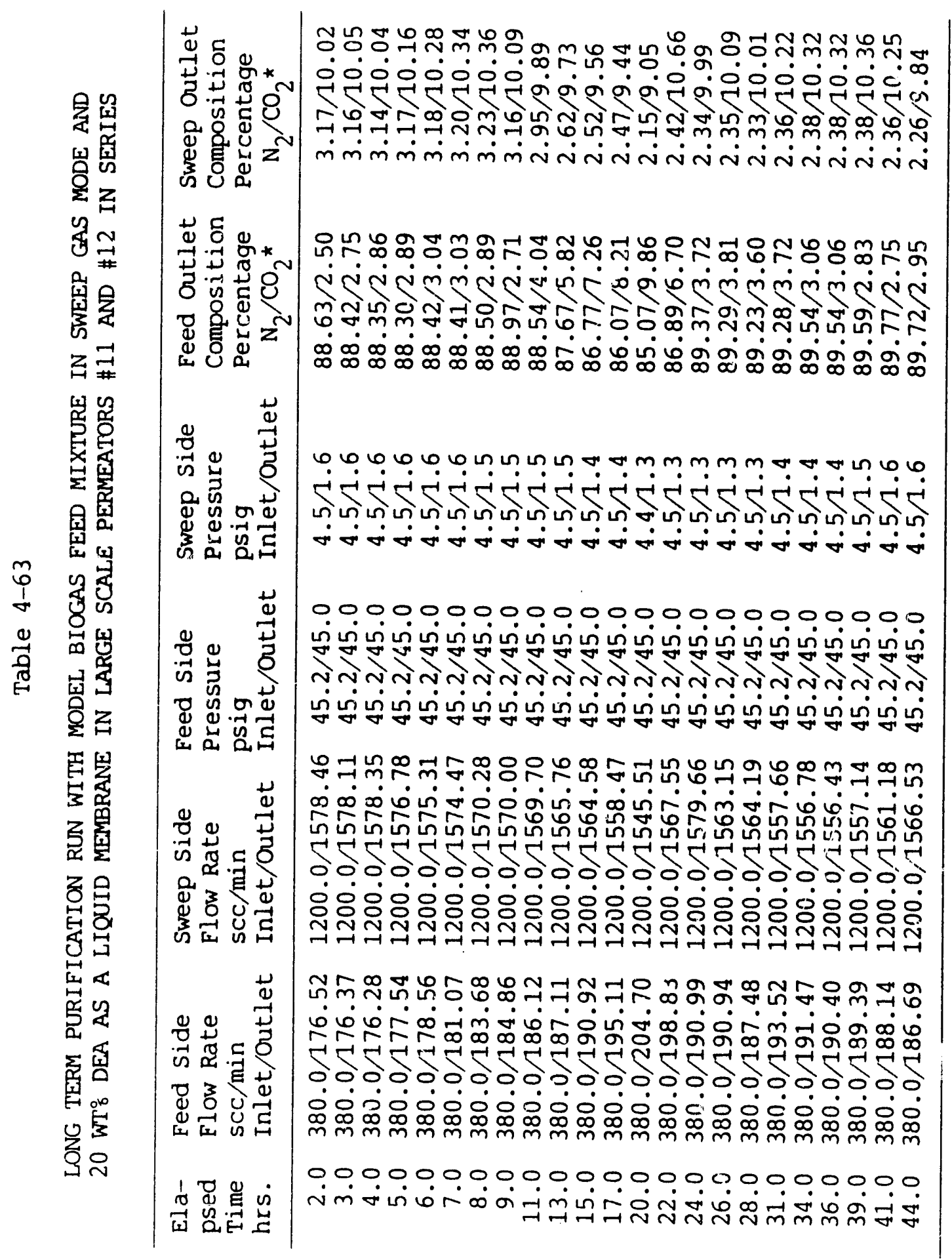




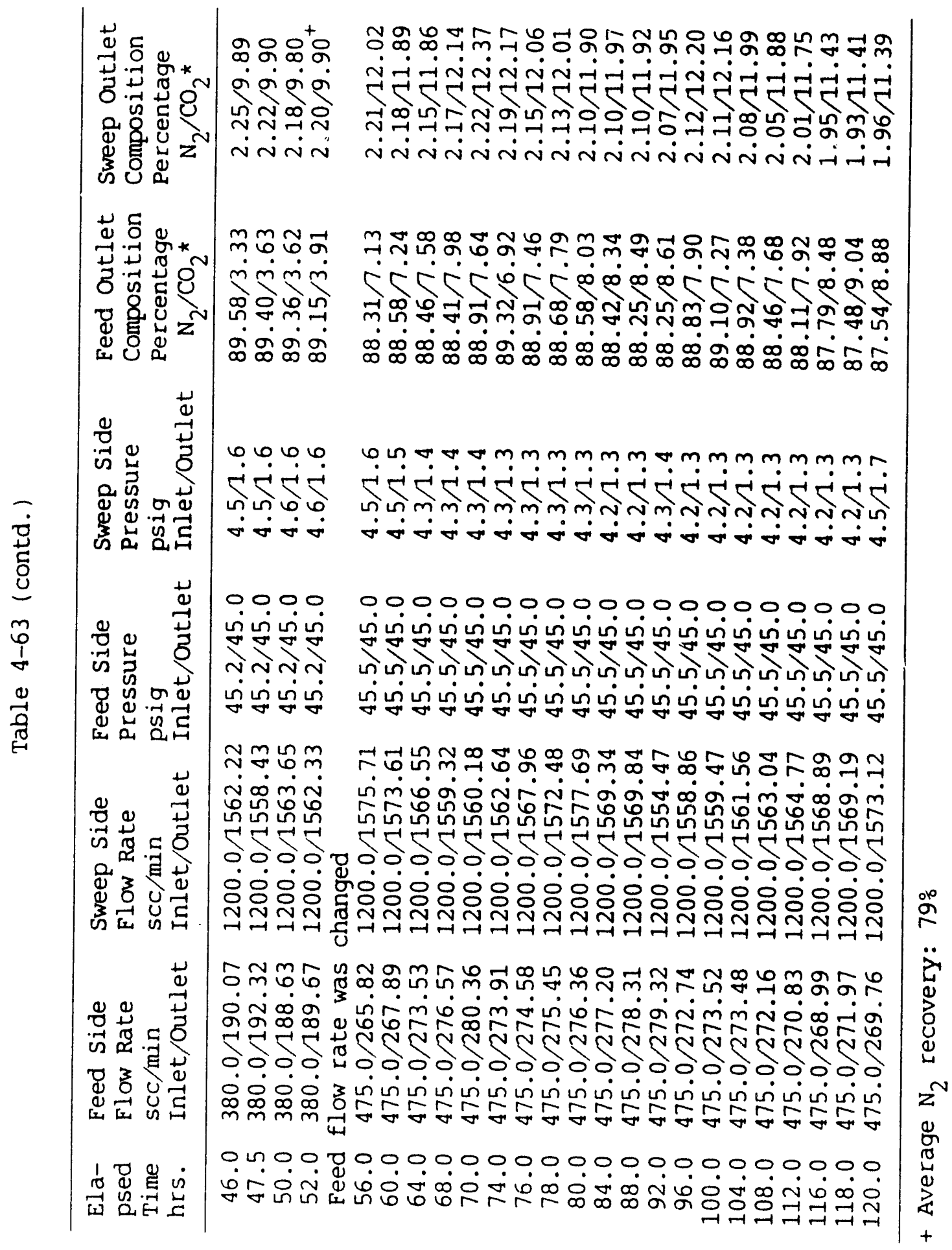




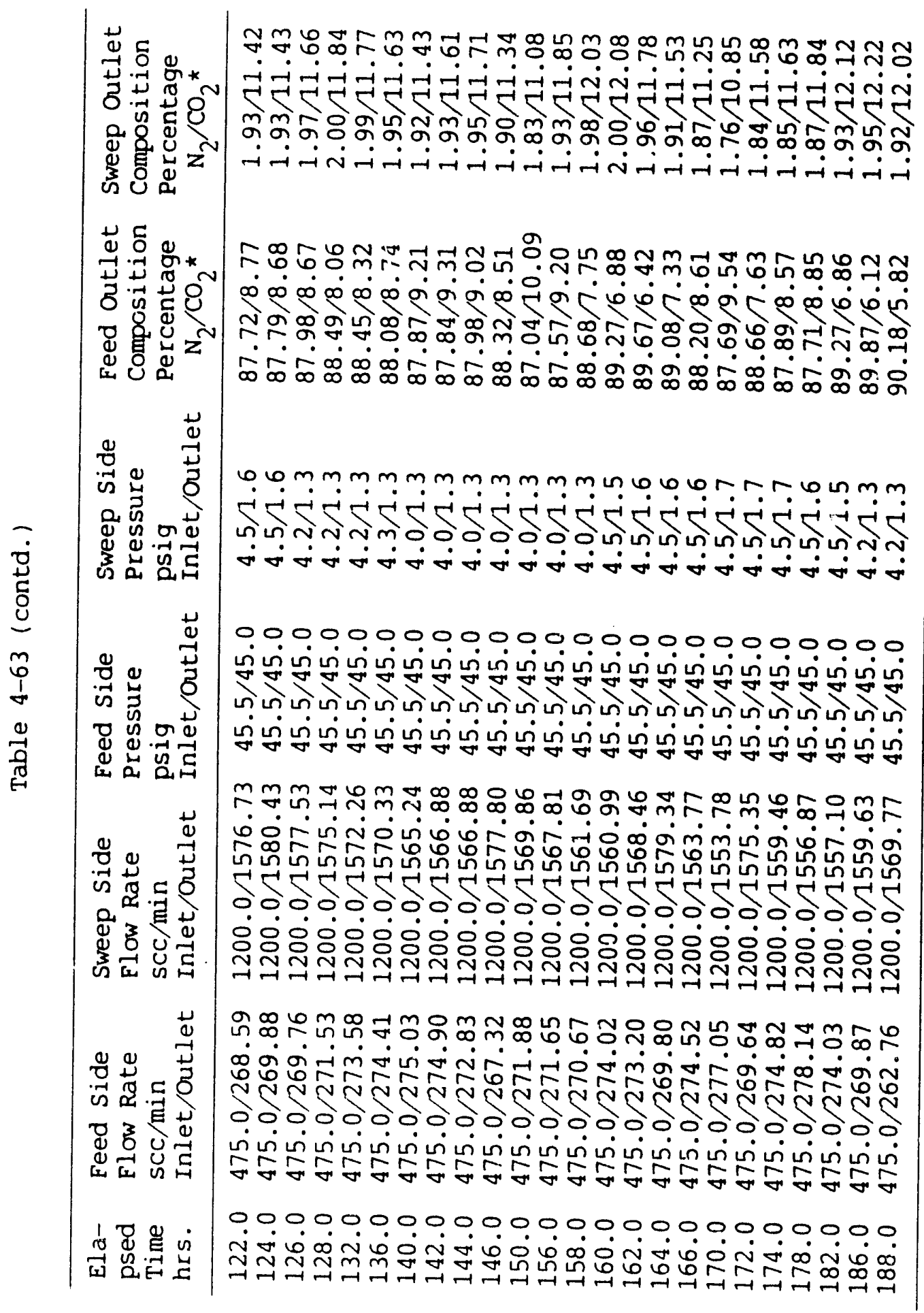




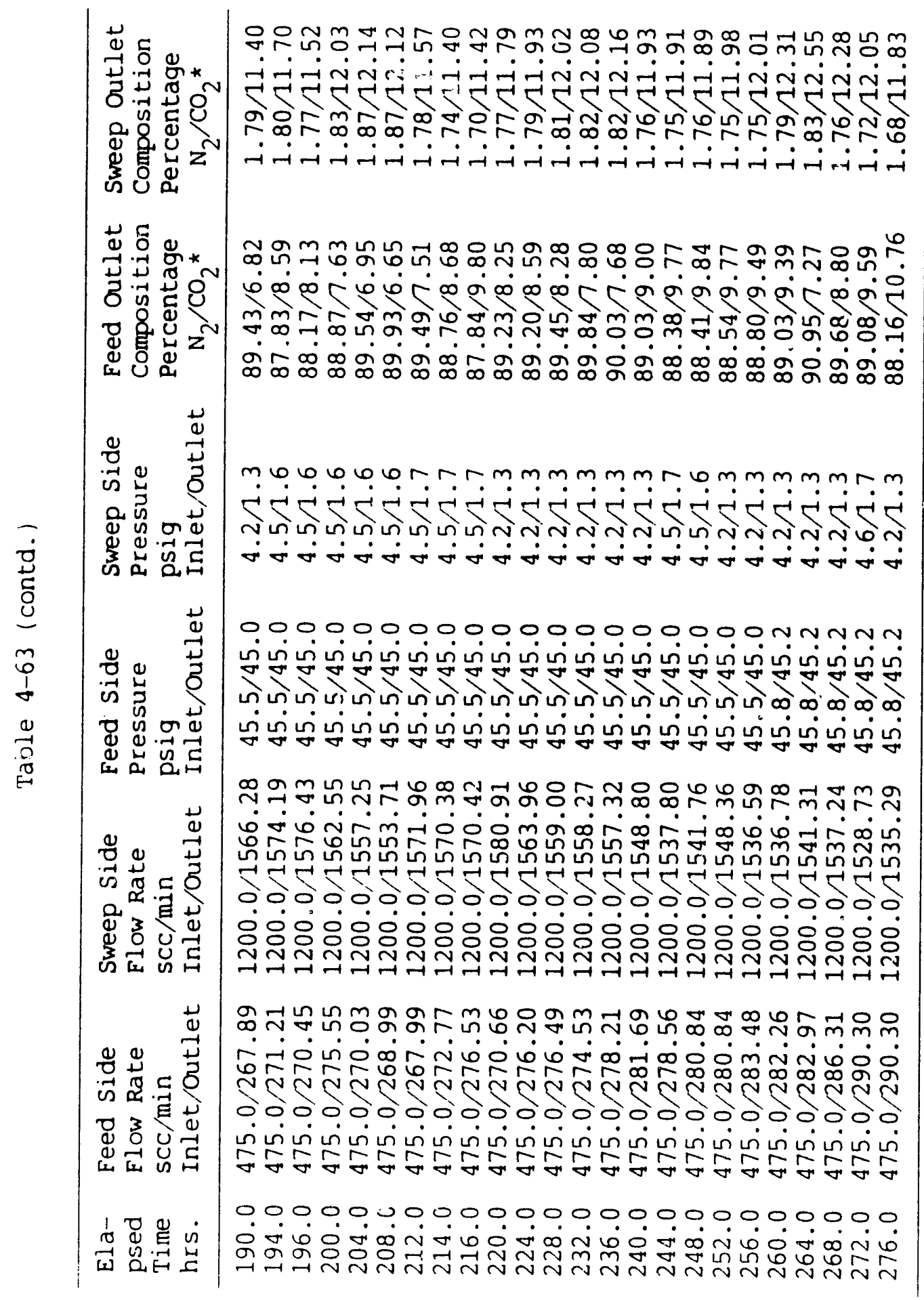




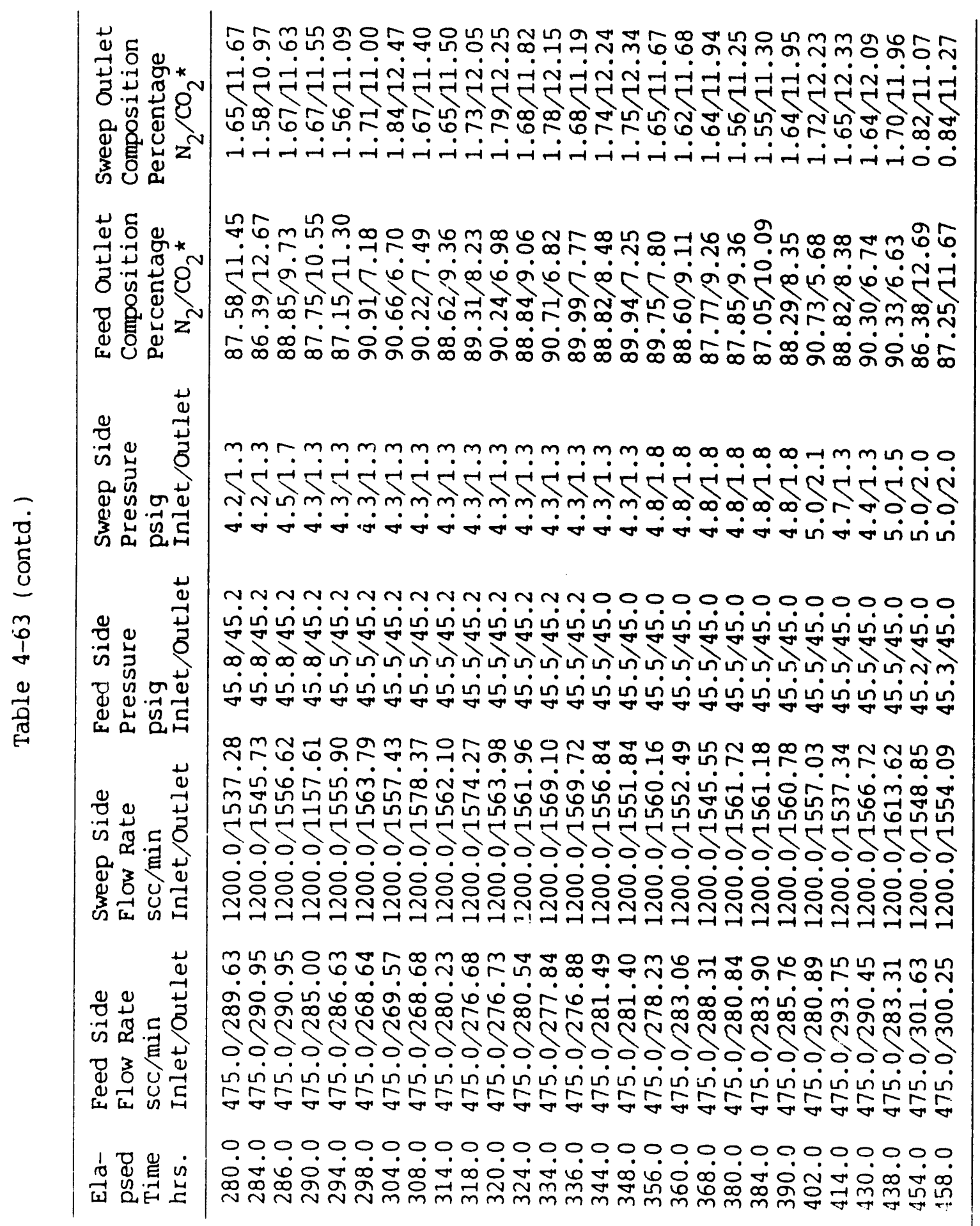




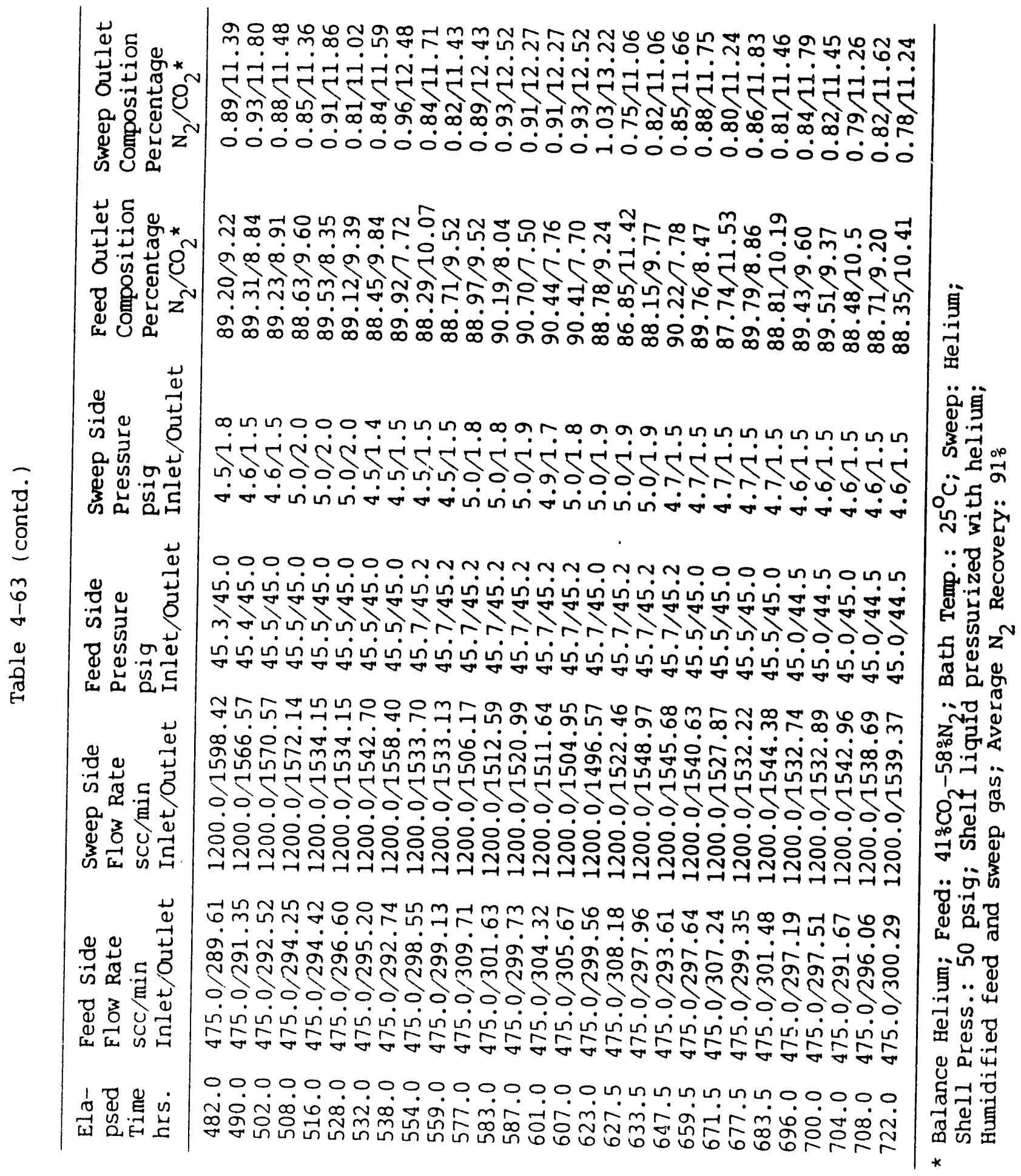




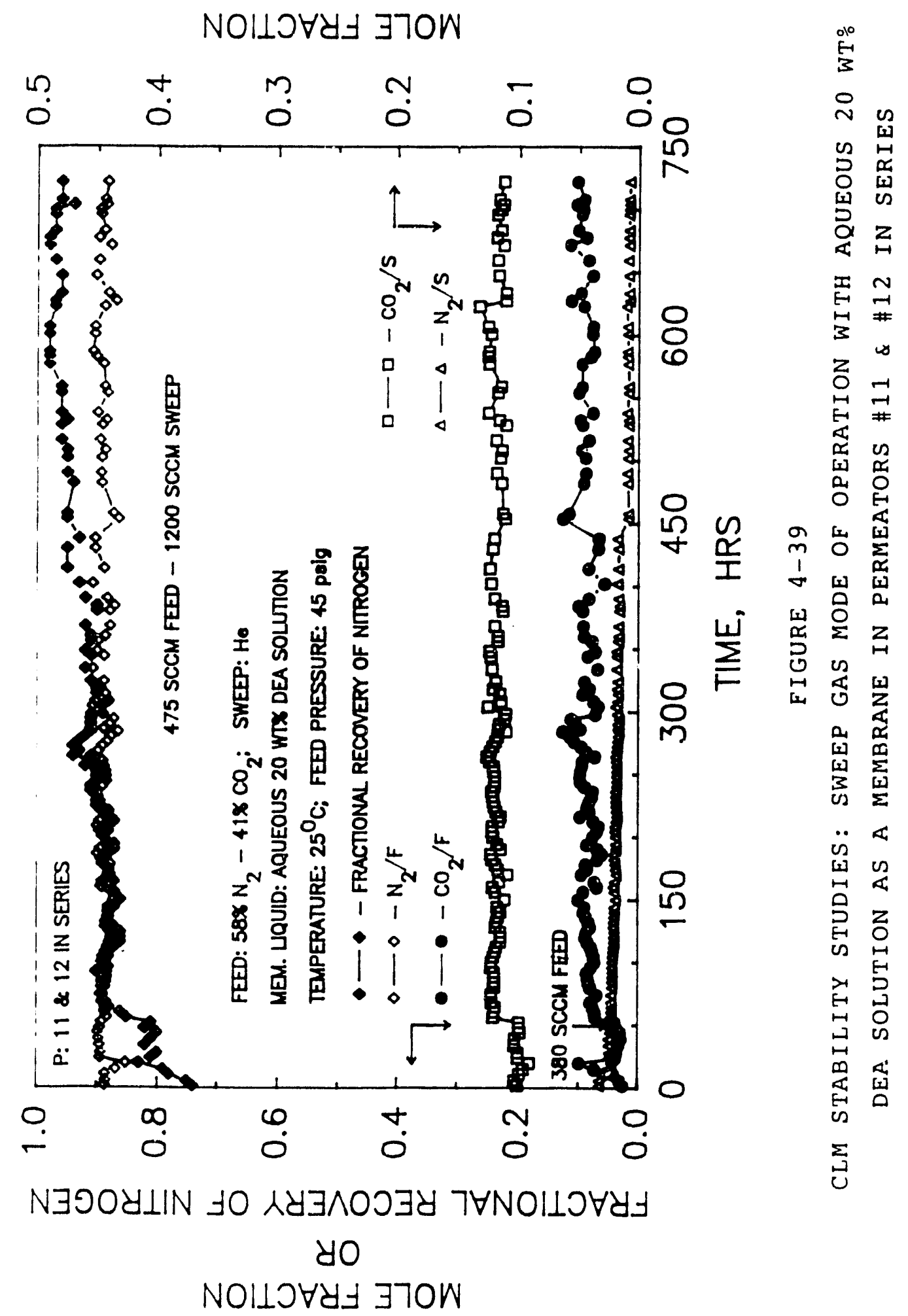


Table 4-64. Here the purified feed gas contained about $24 \% \mathrm{CO}_{2}$ and a $\mathrm{N}_{2}$ recovery of $99 \%$ was obtained. In the second experiment, reported in Table 4-65, a feed gas mixture of $4 \% \mathrm{CO}_{2}$ and $96 \% \mathrm{~N}_{2}$ was used at about atmospheric pressure (problem posed by Permapure Products. Inc.). Here the purified gas contained only about $1 \% \mathrm{CO}_{2}$.

20 wt DEA Solution as Liquid Membrane and $\mathrm{CO}_{2}-\mathrm{CH}_{4}$ Gas Mixture as Feed

Long-term separation studies were carried out over a period of 10 days in Permeator \#11 using a gas mixture containing methane and carbon dioxide. The feed gas composition, as determined by $\mathrm{GC}$ analysis was $44 \% \mathrm{CO}_{2}-56 \% \mathrm{CH}_{4} \cdot$ Pure $\mathrm{N}_{2}$ gas was used as a sweep gas.

The feed gas mixture as well as the sweep gas stream were thoroughly humidified as an aqueous solution of $20 \mathrm{wt} \%$ DEA was utilized as the liquid membrane. The gas stream humidities, as indicated by the humidity probes, were very close to $100 \%$. Two membrane humidifiers were needed in the sweep line whereas one humidifier in the feed line was sufficient to raise the gas stream humidities to be level of $100 \%$. The bath temperature was kept at $25^{\circ} \mathrm{C}$. However, the bath temperature varied considerably during the ten-day run due to the fluctuations in the surrounding temperature. During the evenings and weekends, the air conditioning system was essentially off.

Table 4-66 indicates the flow rates and pressures of various streams. The reaction between $\mathrm{CO}_{2}$ and $\mathrm{DEA}$ is strongly dependent on the temperature. The $\mathrm{CO}_{2}$ composition in the purified gas stream varied from 12 to $24 \%$. This variation is mostly due to the variation in temperature of the system. The pressure drop in the feed stream was negligible and that of the sweep stream was only about 5 psi for the flow rates used in this study. The average methane recovery was close to $94 \%$. The composition of the feed and sweep outlet streams and the recovery of methane gas for the entire period is shown in Figure $4-40$.

The results very strongly support the contention that long-term separation of $\mathrm{CO}_{2}$ can be carried out efficiently with a high methane recovery in a largescale hollow-fiber-contained liquid membrane permeator having a $20 \mathrm{wt} \%$ DEA in water as a liquid membrane. Higher purification can be achieved with higher 


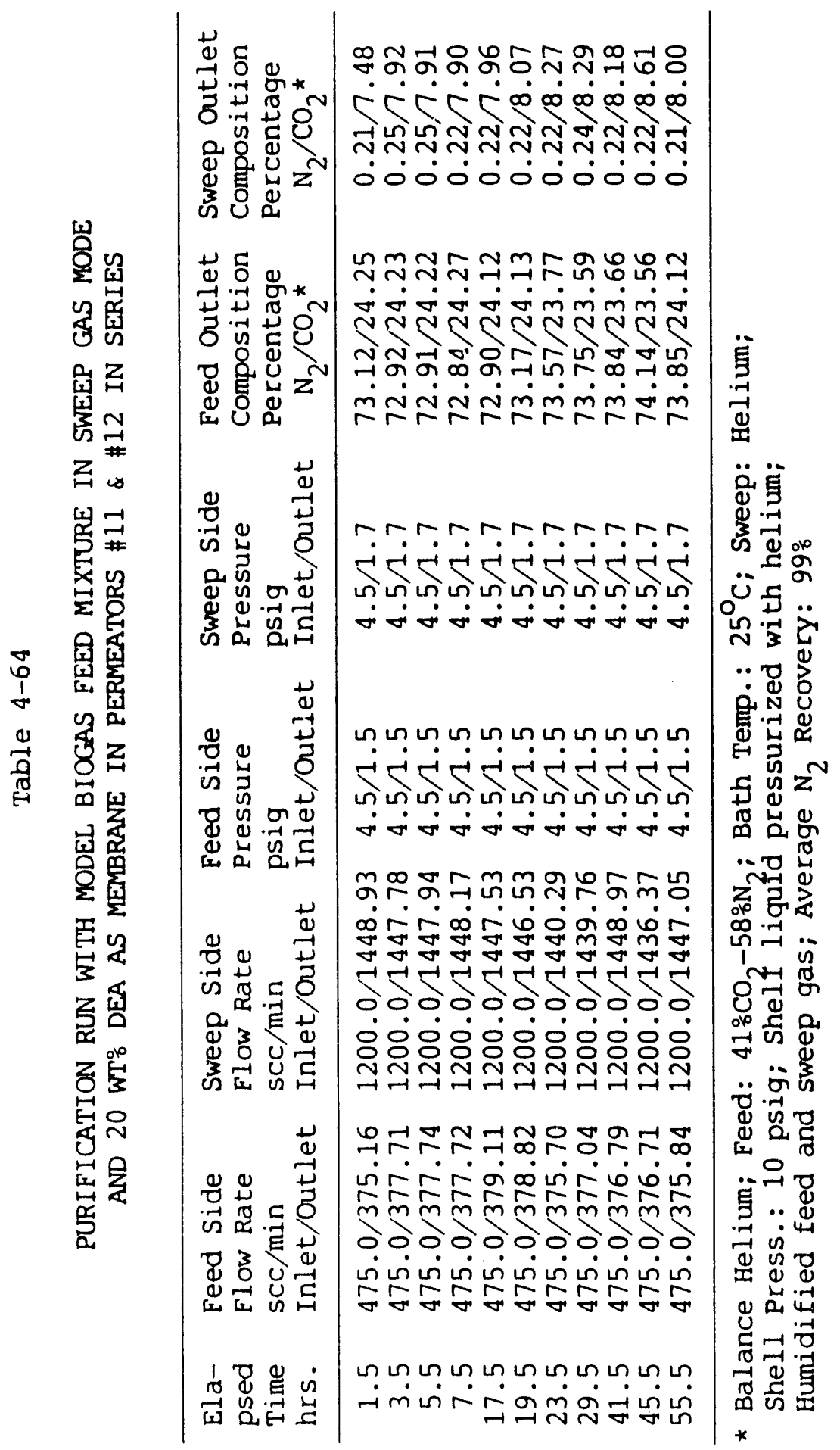




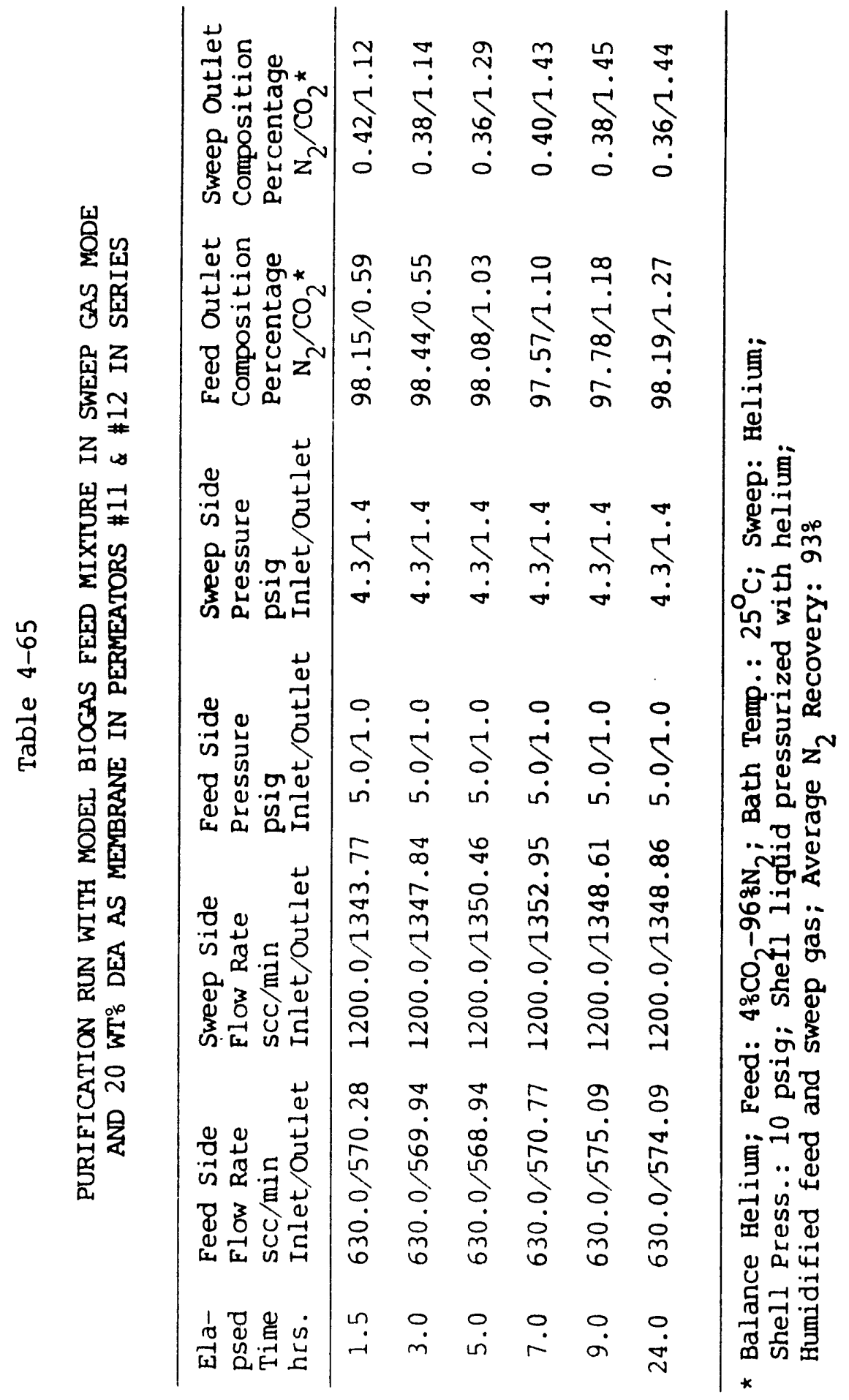




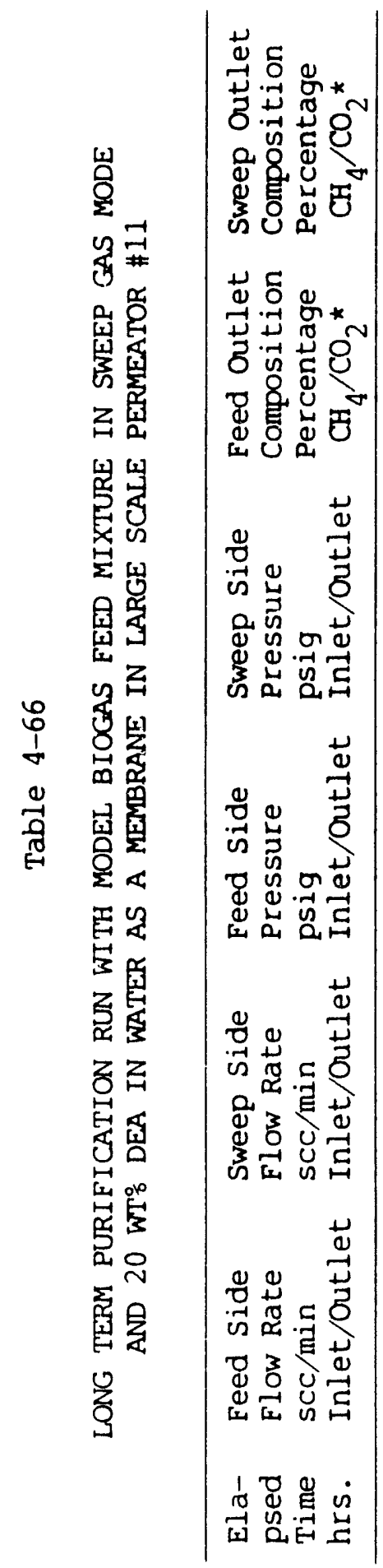

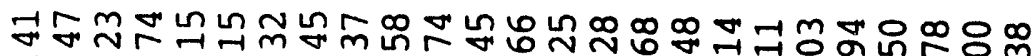

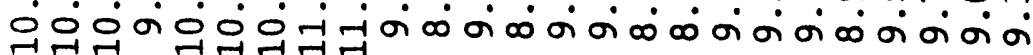
एरत सरतस

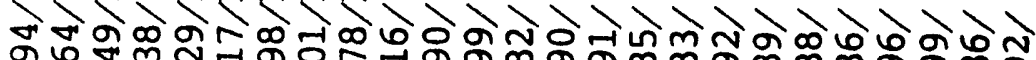

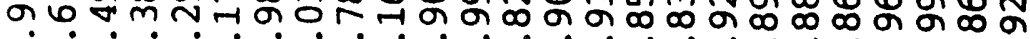

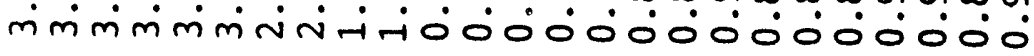

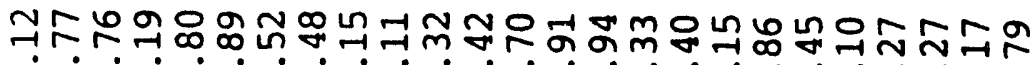

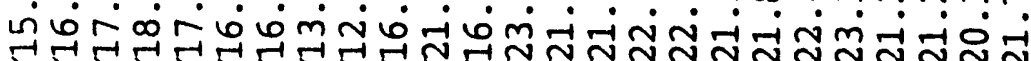

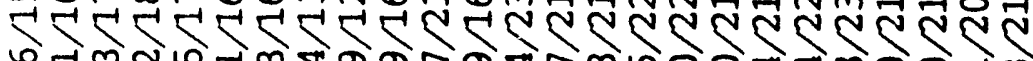

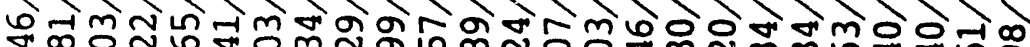
œ ․ అ

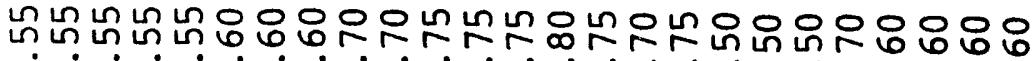

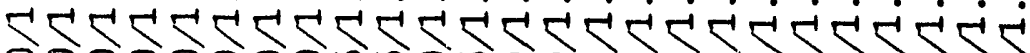

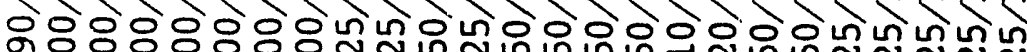
政

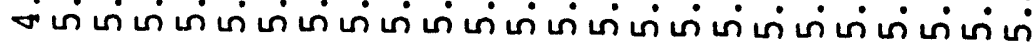

0000000000000000000000000

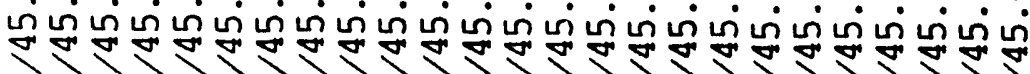

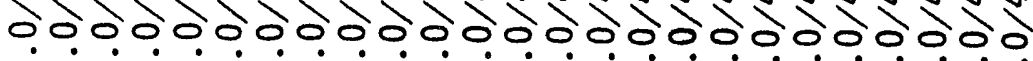

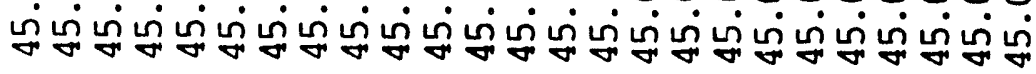

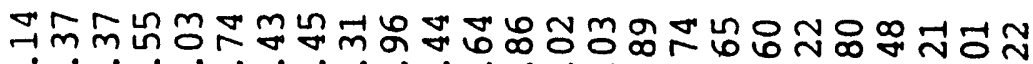
ம்

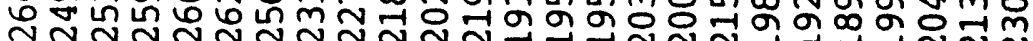

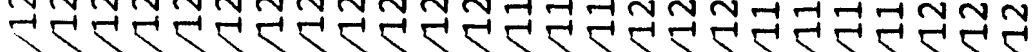

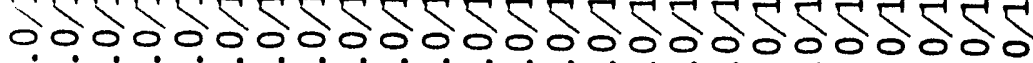

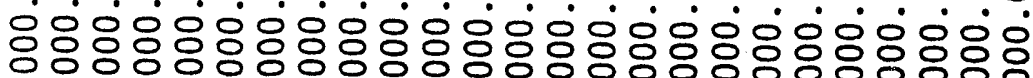

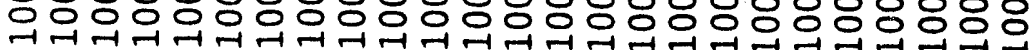

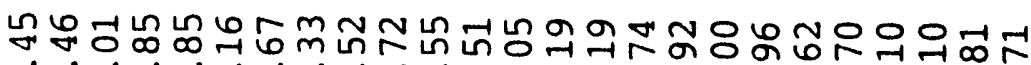

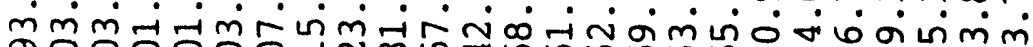

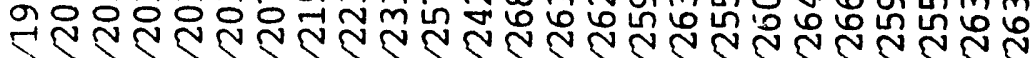

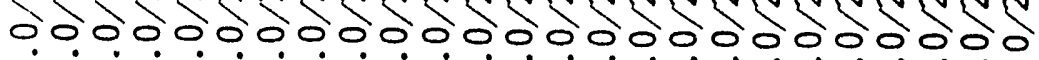
í $\dot{0} \dot{0} \dot{0} \dot{0} \dot{0} \dot{0} \dot{0} \dot{0} \dot{0} \dot{0} \dot{0} \dot{0} \dot{0}$

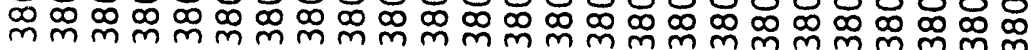
o o o un un o o o un un o o in o o ino o un un un un un un un

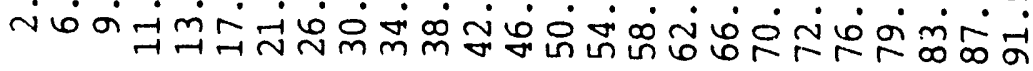




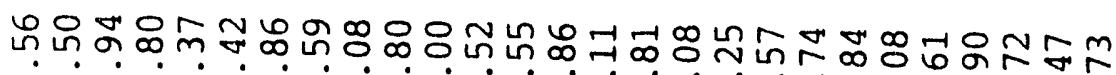
б

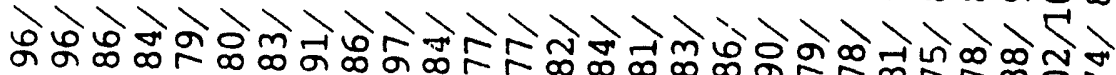
の. 000000000000000000000000.

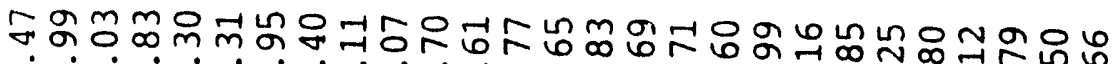

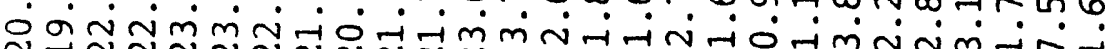

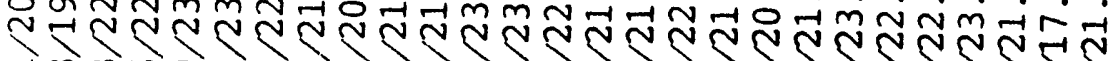
ले

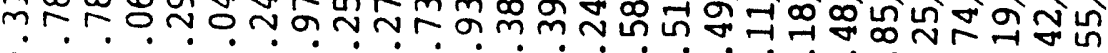
ón大

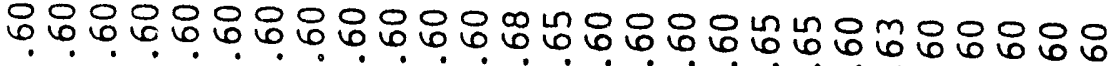

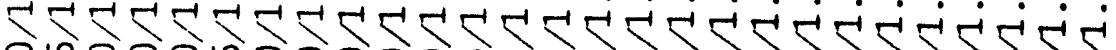

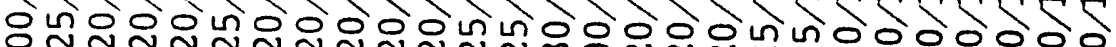

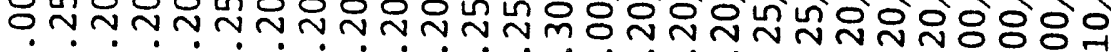
v

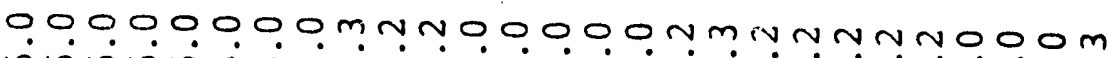

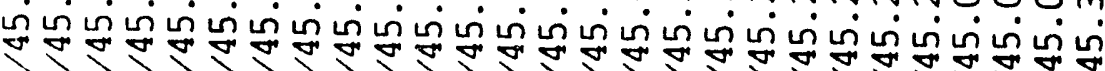

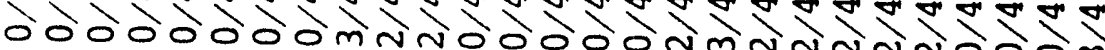
.

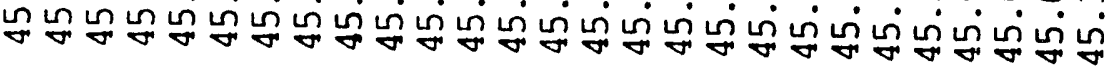

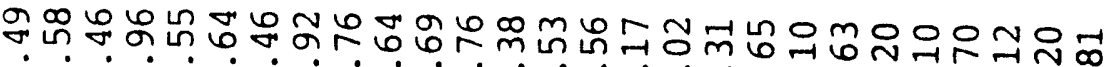

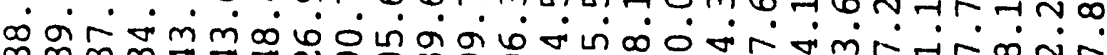
mmm $m$ ॠ

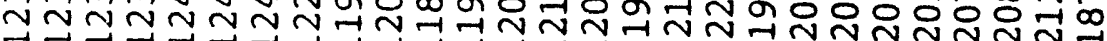
तन

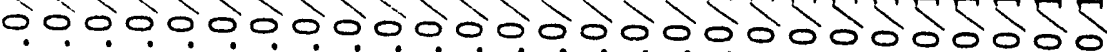

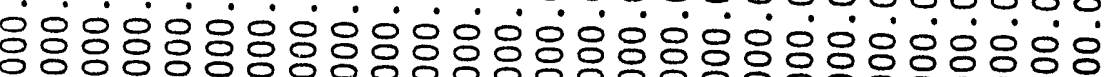
s.ำง

ฌ निंतिं ஸी त्रN

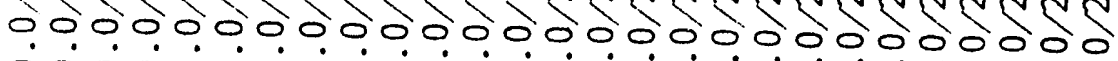
o o o o ó

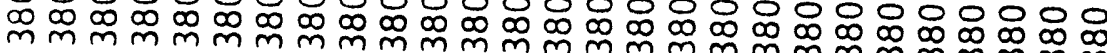

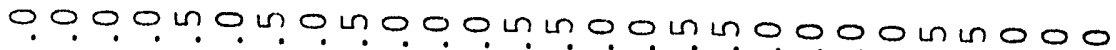
जू ڤं $\rightarrow$ - 


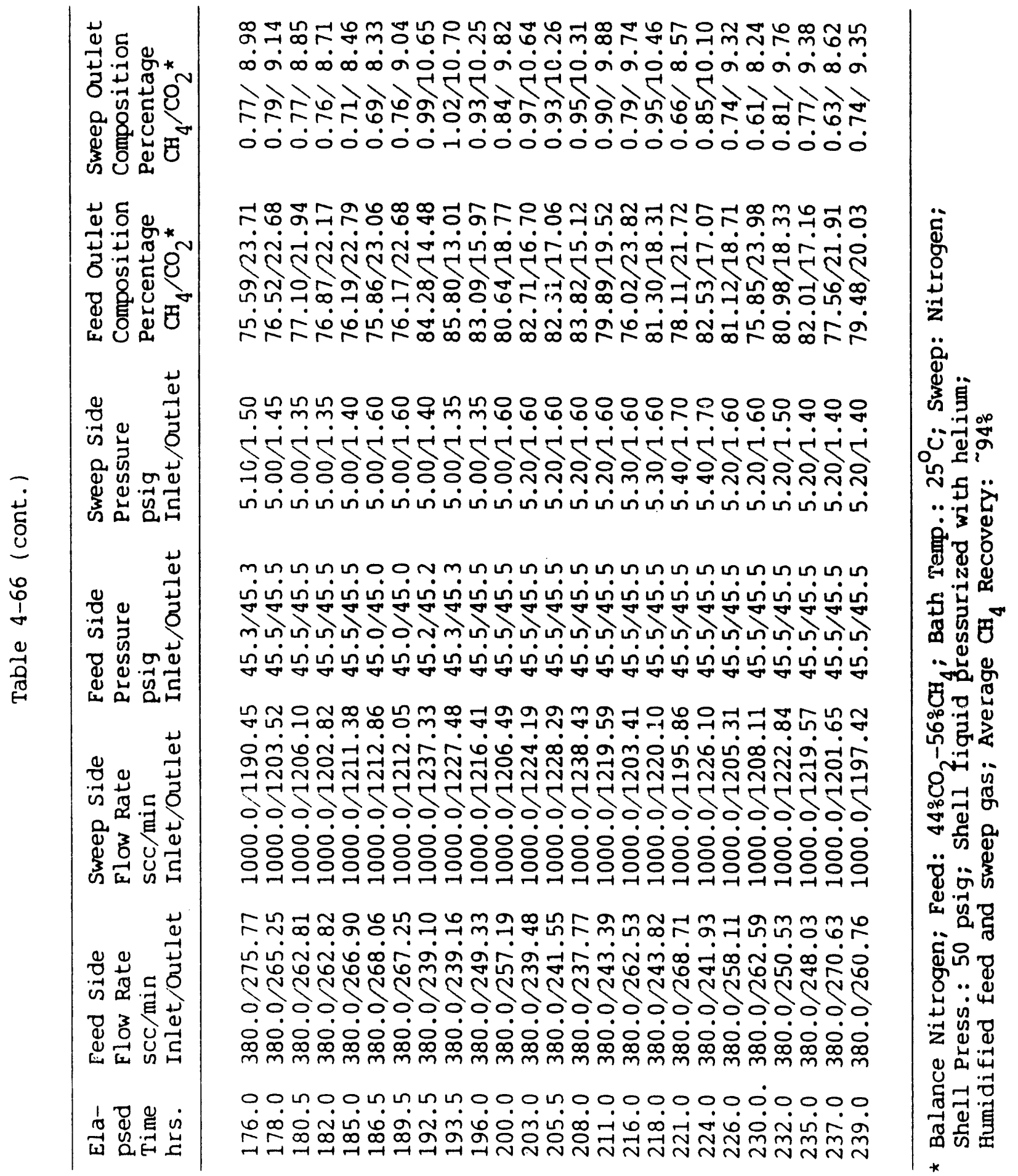




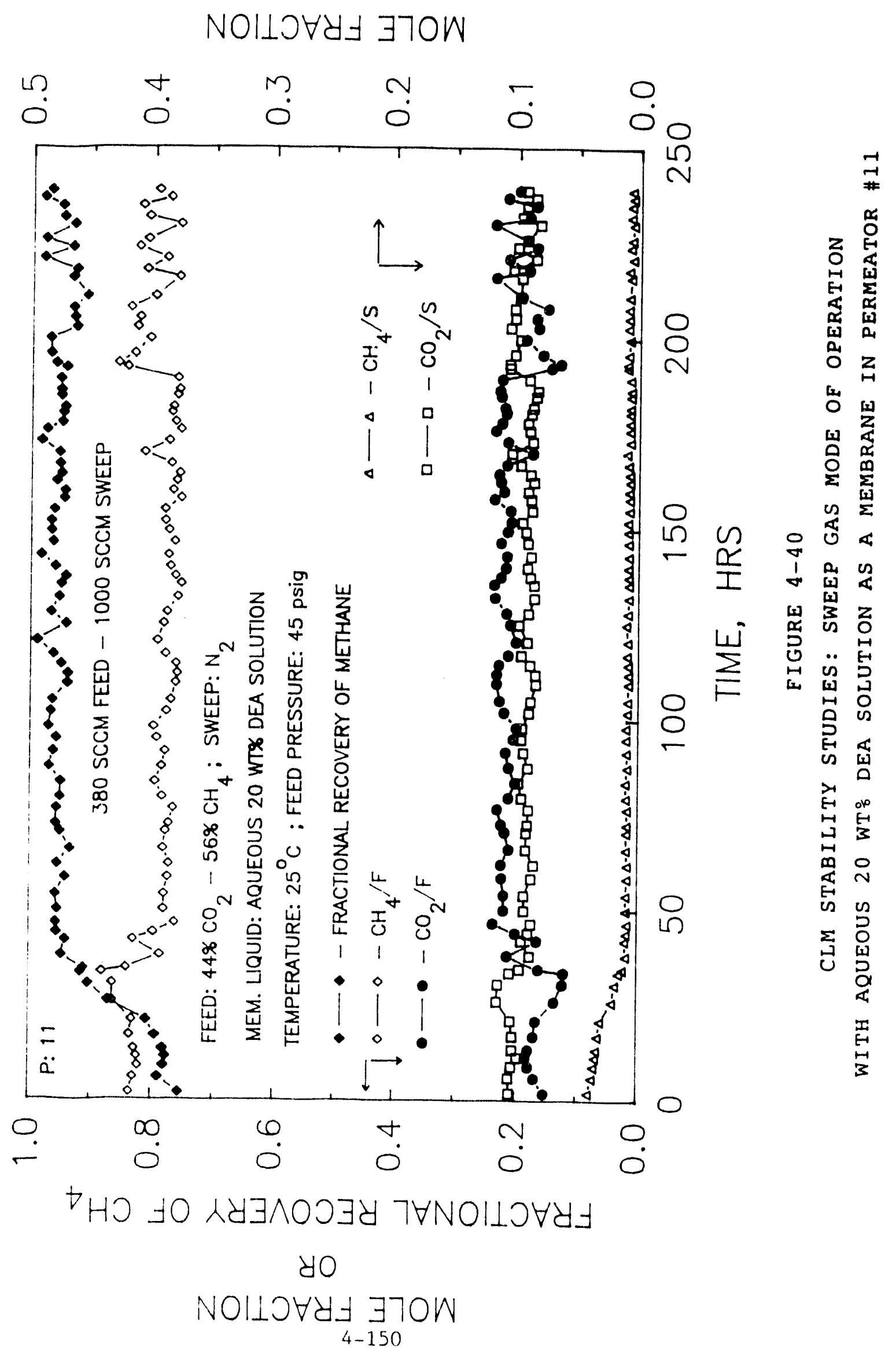


membrane area and a better membrane permeator with a lower thickness of membrane.

BEST PERFORMANCES UNDER DIFPERENT MODES OF OPERATION

It has been shown that the operational flexibility of a permeator increases substantially with the rew HFCLM technology. In the small-scale operation, the new technique was applied to separate a model biogas feed mixture under a variety of operating modes; these include sweep gas, sweep liquid, vacuum and conventional permeation. We have demonstrated that the HFCLM system can be applied successfully under all modes of operation. The best separation performances observed under different operating modes in small-scale operation are summarized in Table 4-67. The operating conditions, purified gas compositions and the recovery in a given operating mode are shown in the table. 


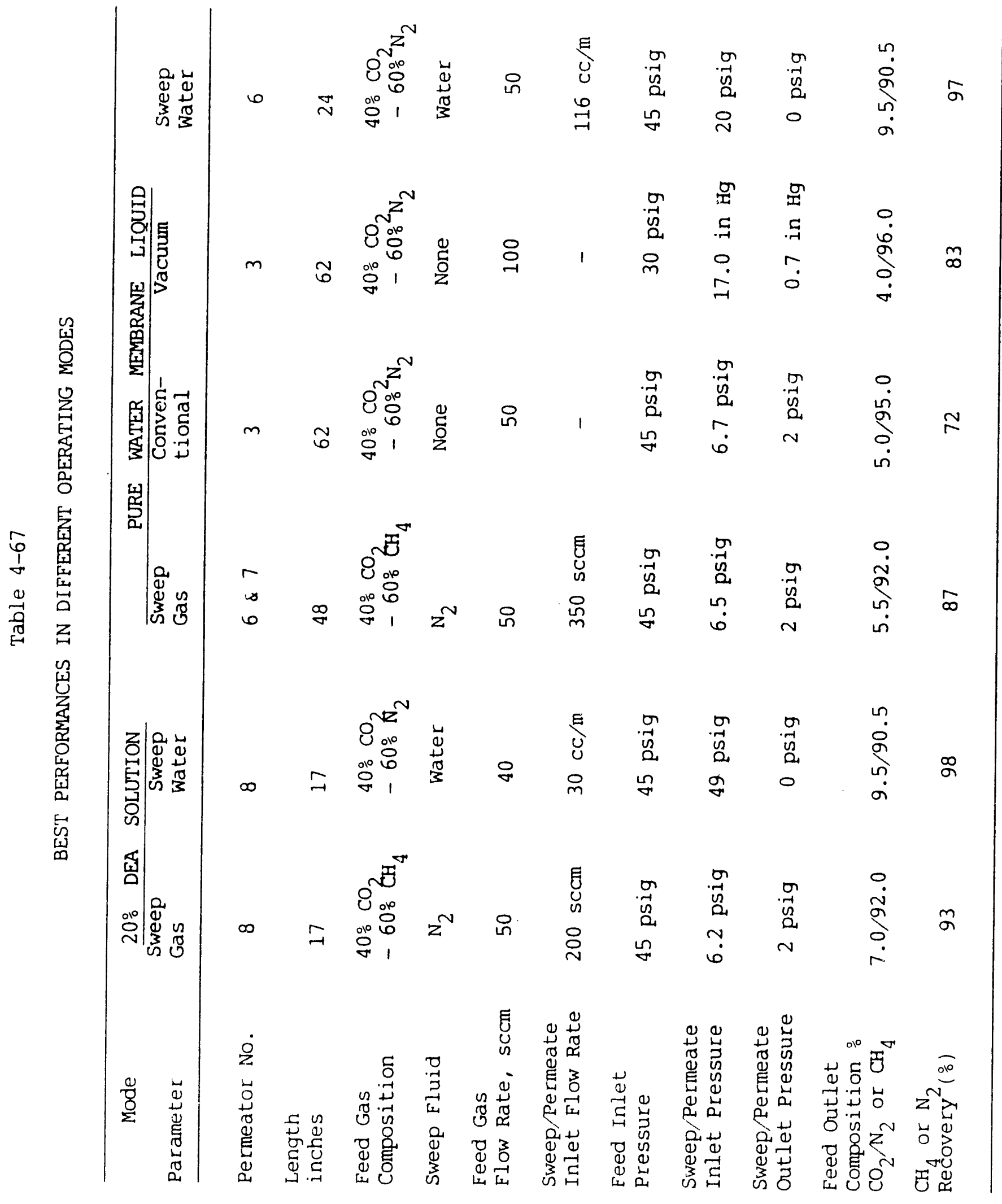




\section{Section 5}

\section{PRELIMINARY RCONOMIC ANALYSIS}

A comparative economic analysis of different modes of HFCLM operation for biogas purification has been carried out here. The purpose of this exercise is to guide our selection of the optimum mode of operation rather than determine the actual cost of purification by such a process. The following five modes have been considered for preliminary calculations:

Mode 1 : Sweep mode of operation with air as sweep gas and 20\% DEA solution as membrane liquid

Mode 2 : Sweep mode of operation with air as sweep gas and water as membrane liquid

Mode 3 : Conventional mode of operation with pure water as membrane Mode 4 : Vacuum mode of operation with pure water liquid membrane Mode 5 : Sweep water mode of operation with pure water membrane

Modes $2,3,4$ and 5 allow a direct comparison of the different modes for pure permeation through a given liquid. For all modes, it was considered that 1 million std cft/day (MSCFD) of biogas (composition: $40 \% \mathrm{CO}_{2}-60 \% \mathrm{CH}_{4}$ ) had to be upgraded to pipeline quality. The operating parameters, utilized for preliminary analysis, are summarized in Table 5-1. These projections were based on our experimental observations.

The estimated production cost under various modes (i.e., 1, 2, 3, 4 and 5) are shown in Tables $5-2,5-3,5-4,5-5$ and 5-6, respectively. All discussions pertain to a product gas purity of $95 \%$ methane. For simplicity, raw feed gas was assumed to be available at no cost. The estimated cost of membrane area, utility etc. are shown at the bottom of the Table 5-2. The same values were used for production cost calculation of other modes. The sizes and costs of compressor, blower, pump and vacuum pump were determined from Peters and Timmerhaus (1980), Evans (1979) and Matley (1984, 1977).

It is obvious that the cost of purification is the lowest for $20 \%$ aqueous DEA solution. It is also obvious that the capital as well as operating cost of 
TABLE 5-1

SUMMARY OF PARAMETERS FOR A COMPARATIVE ECONOMIC ANALYSIS OF DIFFERENT OPERATING MODES

\begin{tabular}{|c|c|c|c|c|c|}
\hline $\begin{array}{l}\text { Mode } \\
\text { Parameter }\end{array}$ & $\begin{array}{l}\text { Sweep Air } \\
\text { with } 20 \% \\
\text { DEA Sol. }\end{array}$ & $\begin{array}{l}\text { PURE } \\
\text { Sweep } \\
\text { Air }\end{array}$ & $\frac{\text { WATER } M}{\substack{\text { Conven- } \\
\text { tional }}}$ & $\frac{\text { MEMBRANE }}{\text { Vacuum }}$ & $\begin{array}{l}\text { LIQUID } \\
\text { Sweep } \\
\text { Water }\end{array}$ \\
\hline $\begin{array}{l}\text { Feed Gas } \\
\text { Processing } \\
\text { Capacity } \\
(\text { MSCFD) }\end{array}$ & 1 & 1 & 1 & 1 & 1 \\
\hline $\begin{array}{l}\text { Sweep } \\
\text { Fliuid }\end{array}$ & Air & Air & None & None & Water \\
\hline $\begin{array}{l}\text { Feed Inlet } \\
\text { Pressure } \\
(\text { Atm) }\end{array}$ & 4 & 4 & 4 & 4 & 4 \\
\hline $\begin{array}{l}\text { Sweep/Permeate } \\
\text { Outlet Pressure } \\
(A t m)\end{array}$ & 1 & 1 & 1 & 0.07 & 1 \\
\hline $\begin{array}{l}\text { Sweep/Permeate } \\
\text { Inlet Pressure } \\
(A t m)\end{array}$ & 1.5 & 3 & $i$ & 0.5 & 3 \\
\hline $\begin{array}{l}\text { Sweep/Permeate } \\
\text { Inlet } \\
\text { Flow Rate }\end{array}$ & 4 MSCFD & 3 MSCFD & - & - & $1700 \mathrm{CFM}^{2}$ \\
\hline $\begin{array}{l}\text { Maximum } \\
\text { Impurity } \\
\text { in Product } \\
(\%)\end{array}$ & 5 & 5 & 5 & 5 & 5 \\
\hline $\begin{array}{l}\text { Methane } \\
\text { Recovery } \\
(\%)\end{array}$ & 95 & 80 & 70 & 85 & 95 \\
\hline
\end{tabular}


TABLE 5-2

ECONOMIC CHARACTERISTICS OF SWEEP GAS MODE OF OPERATION WITH 20\% DEA SOLUTION AS MEMBRANE

\begin{tabular}{|c|c|c|c|}
\hline I tem & Size & Number & $\begin{array}{c}\text { Cost } \\
(\$ 1,000)\end{array}$ \\
\hline
\end{tabular}

1. Fixed Capital Cost

1.1 Process Equipment

- Membrane Module $1 \frac{1}{2}^{\prime}(\mathrm{L}) \times 16^{\prime \prime}(\mathrm{D}) \quad 100$ 550

- Single Stage Gas $100 \mathrm{hp}$ 1 70 Compressor with Gas Fired Engine

- Centrifugal $100 \mathrm{hp}$ 1 Blower

- Subtotal of 1.1

1.3 Total of 1.1 and 1.2

2. Manufacturing Cost per Year

2.1 Utility ${ }^{c}$

2.2 Labor $^{d}$

3. Total Product Price ${ }^{e}$ per Year

a $\$ 1.00$ per $\mathrm{ft}^{2}$ of membrane area

$b$ estimated by multiplying the item of 1.1 by $2 / 3$

c $\$ 0.088$ per $\mathrm{KW}-\mathrm{Hr}$

d $\$ 12.00$ per Man-Hqur, 2 persons

e $\$ 0.65$ per $100 \mathrm{ft}^{3}$ of $95 \% \mathrm{CH}_{4}$ natural gas 
TABLE 5-3

ECONOMIC CHARACTERISTICS OF SWEEP GAS MODE OF OPERATION

\begin{tabular}{|c|c|c|c|}
\hline I tem & Size & Number & $\begin{array}{c}\text { Cost } \\
(\$ 1,000)\end{array}$ \\
\hline
\end{tabular}

1. Fixed Capital Cost

1.1 Process Equipment

- Membrane Module

$5^{\prime}(\mathrm{L}) \times 16^{\prime \prime}(\mathrm{D})$

$100 \mathrm{hp}$

- Single Stage Gas Compressor with

Gas Fired Engine

- Centrifugal Blower

- Subtotal of 1.1

$300 \mathrm{hp}$
48

900

1

70

1

25

995

1.2 Piping, Instrumentation and Installation

1.3 Total of 1.1 and 1.2

2. Manufacturing Cost per Year

2.1 Utility

2.2 Labor

2.3 Total of 2.1 and 2.2 $\underline{440}$

3. Total Product Price per Year 
TABLE 5-4

ECONOMIC CHARACTERISTICS OF CONVENTIONAL MODE OF OPERATION

\begin{tabular}{|c|c|c|c|}
\hline I tem & Size & Number & $\begin{array}{c}\text { Cost } \\
(\$ 1,000)\end{array}$ \\
\hline
\end{tabular}

1. Fixed Capital Cost

1.1 Process Equipment

$\begin{array}{lcrr}\text { - Membrane Module } & \text { S'(L) X 16"(D) }^{\prime \prime} & 96 & 1,800 \\ \text { - Single Stage Gas } & 100 \mathrm{hp} & 1 & 70 \\ \text { Compressor with } & & & \\ \text { Gas Fired Engine } & & & \underline{1,870} \\ \text { - Subtotal of } 1.1 & & & \end{array}$

1.2 Piping, Instrumentation and Installation

1.3 Total of 1.1 and 1.2

$\underline{1,247}$

3,117

2. Manufacturing Cost per Year

2.1 Utility

$\underline{58.4}$

2.2 Labor

210.0

2.3 Total of 2.1 and 2.2

268.4

3. Total Product Price per Year

$\underline{1,050}$ 
TABLE 5-5

ECONOMIC CHARACTERISTICS OF VACUUM MODE OF OPERATION

\begin{tabular}{|c|c|c|c|}
\hline Item & Size & Number & $\begin{array}{c}\text { Cost } \\
(\$ 1,000)\end{array}$ \\
\hline
\end{tabular}

1. Fixed Capital Cost

1.1 Process Equipment

- Membrane Module $\quad 5^{\prime}(\mathrm{L})$ X 16"(D) 48

- Single Stage Gas $100 \mathrm{hp} \quad 110$

Gas Fired Engine

$\begin{array}{llll}\text { - Reciprocating } & 250 \mathrm{hp} & 1 & 48 \\ \text { Mechanical Vacuum } & & & \end{array}$

Pump with Explosion

Proof Motor

- Subtotal of 1.1

1.2 Piping, Instrumentation

and Installation

1.3 Total of 1.1 and 1.2

2. Manufacturing Cost per Year

2.1 Utility

201

2. 2 Labor

210

2.3 Total of 2.1 and 2.2

411

3. Total Product Price per Year

$\underline{1,270}$ 
TABLE 5-6

ECONOMIC CHARACTERISTICS OF SWEEP WATER MODE OF OPERATION

\begin{tabular}{|c|c|c|c|}
\hline I tem & Size & Number & $\begin{array}{c}\text { Cost } \\
(\$ 1,000)\end{array}$ \\
\hline
\end{tabular}

1. Fixed Capital Cost

1.1 Process Equipment

- Membrane Module 2'(L) X 16"(D) 125

- Single Stage Gas $\quad 100 \mathrm{hp} \quad 110$

Compressor with

Gas Fired Engine

- Cast Steel Casing $200 \mathrm{hp} \quad 2 \quad 30$

Inline Horizontal

Motor and

Centrifugal pump

- Subtotal of 1.1

1.2 Piping, Instrumentation

and Installation

1.3 Total of 1.1 and 1.2

2. Manufacturing Cost per Year

2.1 Utility

2.2 Labor

210

2.3 Total of 2.1 and 2.2

3. Total Product Price per Year 
purification for sweep air (Table 5-3), vacuum mode (Table 5-5) are quite close. However, the sweep water mode yields higher product price because of higher methane recovery. 


\section{Section 6}

\section{INTERACTIONS WITH CORPORATIONS REGARDING HPCLM TECHNOLOGY}

As part of project task $\mathrm{H}$ requiring the contractor to acquaint corporations about the value and possible usefulness of this technology, a number of corporations have been contacted during 1988-1989. The objectives included possible commercialization of the hollow-fiber-contained liquid membrane technique for separation of gas mixtures in general as well as application of this technology for biogas separation $/ \mathrm{CO}_{2}$ elimination in particular. Many corporations were sent a copy of our first paper (AIChE J., 34(7), 1135-1148, 1988) along with the information that a U.S. patent has been issued to us for both process concepts and apparatus (US Patent N0. 4,750,918, dated June 14, 1988 ).

The following corporations have expressed an interest/or continuing discussions with us/or visited our laboratories for licensing purposes or collaborative activities leading to licensing.

\section{COMMENTS}

1. Permapure Products, Inc.

They have reviewed

8 Executive Dr. our patent and papers.

Toms River, NJ 08754

Visited Stevens on

Contact: Mr. Jack Kertzman

August 17, 1989.

Tel.No.: 201-244-0010

Discussions continuing for possible medical application of the technique for removing $\mathrm{CO}_{2}$. An experiment has been carried out to simulate the exact conditions of application.

2. Air Products and Chemicals, Inc. Visited Stevens on

Allentown, PA, 18195

August 8, 1989.

Contact: Dr. Daniel V. Laciak

Further visits

Dr. V. Raman

planned.

Tel.No.: (215)481-7889

$$
\text { (215) } 481-8336
$$

They are thoroughly familiar with our patents and published papers. They are 
experimenting with HFCLM modules in their laboratory. These modules were purchased from Hoechst Celanese Corporation, Charlotte, N.C., 28224. They have approached us for making larger scale modules (not available from Hoechst Celanese at this time). If their evaluation is successful, chances for licensing technology to them is very high. It has been directly stated to us by Dr. Laciak.

\section{Hoechst Celanese Corporation Separations Products Division \\ 13800 S. Lakes Dr. \\ They are quite familiar with our work at the \\ published level.}

Charlotte, NC, 28224

Contact: Dr. Robert W. Callahan

Tel.No.: (704)588-5310

Hoechst Celanese has started marketing since late 1988 HFCLM modules of small sizes. They have encountered very good response from the market. They have acquired rights from stevens to use these modules for liquid separations in the middle of 1985. However, recently, they have sold a number of these modules also for gas separation (e.g. to Air Products and Chemicals, Inc.). After recent telephone conversations, Hoechst Celanese has indicated that they are going to open conversations with Stevens ( $\mathrm{Dr}$. Stella Manne of Stevens Research nffice) regarding licensing. Two items are of interest in this licensing discussion: (1) Large scale HFCLM permeator fabrication techniques developed under NYSERDA-NYGAS-GRI contract: These techniques are useful for both gas and liquid separations; (2) Licensing of HFCLM technique (process and/or apparatus) patent to Hoechst Celanese for gas separation.

4. Ecolotrol

1300 Shames Drive,

Westbury, NY 11590

Contact: Roger Owens

Tel.No.: 516-997-2211
They have reviewed

our patent and

AIChE J. papers.

We have had several contacts with Ecolotrol. Roger Owens has suggested the problem of removing $\mathrm{CO}_{2}$ from an essentially $40 \% \mathrm{CO}_{2}-60 \% \mathrm{~N}_{2}$ stream coming out at 4-6 inches of water gauge from a denitrification bioreactor. The flow rate is about $35 \mathrm{ft}^{3} / \mathrm{min}$. He would like to know an estimate of cost of removing $\mathrm{CO}_{2}$ and recirculating the $\mathrm{N}_{2}$ back into the bioreactor. One could use air as a 
sweep gas or use a vacuum or use water as a sweep liquid. Experiments have been done recently with helium as a sweep gas and $\mathrm{N}_{2}-\mathrm{CO}_{2}(60-40)$ feed gas in our large scale setup with $20 \%$ aqueous DEA solution as the liquid membrane. The results are excellent. Helium was present only to the extent of $2 \%$ in the purified $\mathrm{N}_{2}$. This indicates that the amount of $\mathrm{O}_{2}$ in purified $\mathrm{N}_{2}$ will be less than $0.3 \%$ if air is used as a sweep gas (required for denitrification). Some calculations will be done and communicated to them. If found reasonable, this may be the basis for onsite studies with a slip stream after their denitrification reactor is ready.

\section{Biothane}

2500 Broadway,

Camden, NJ 08104

Contact: Dr. Robert Sax

Tel.No.: (609)541-3500

6. Purac Engineering

100 Crossways Park West

Woodbury, LI 11797

Contact: William Borkoski

Tel.No.: (302)239-9431
Paper (AIChE J.) sent.

They are supposed to be evaluating the process and will get back to us when ready.

Paper (AIChE J.)

sent. They are

evaluating the process.

Efforts to get this technology licensed out will continue after the completion of the current project. It appears though that a critical stage has been reached. The opening up of licensing discussions with Hoechst Celanese with Air Products and Chemicals Inc. in the backdrop is most promising. Dr. Callahan of Hoechst Celanese indicated that they ought to be satisfying the needs of Air Products and Chemicals and other corporations rather than Stevens building modules for Air Products and Chemicals. In this sense, HFCLM technique is the most desired item in their mind rather than a sperific application. A most recent presentation of ours on the long term stability of larger HFCLM permeators apparently has clinched the remaining issues about this technique. 


\section{Section 7}

\section{NOMENCLATURE}

Sometimes more common units are used for clarity. The actual units used on any occasion are stated in the text.

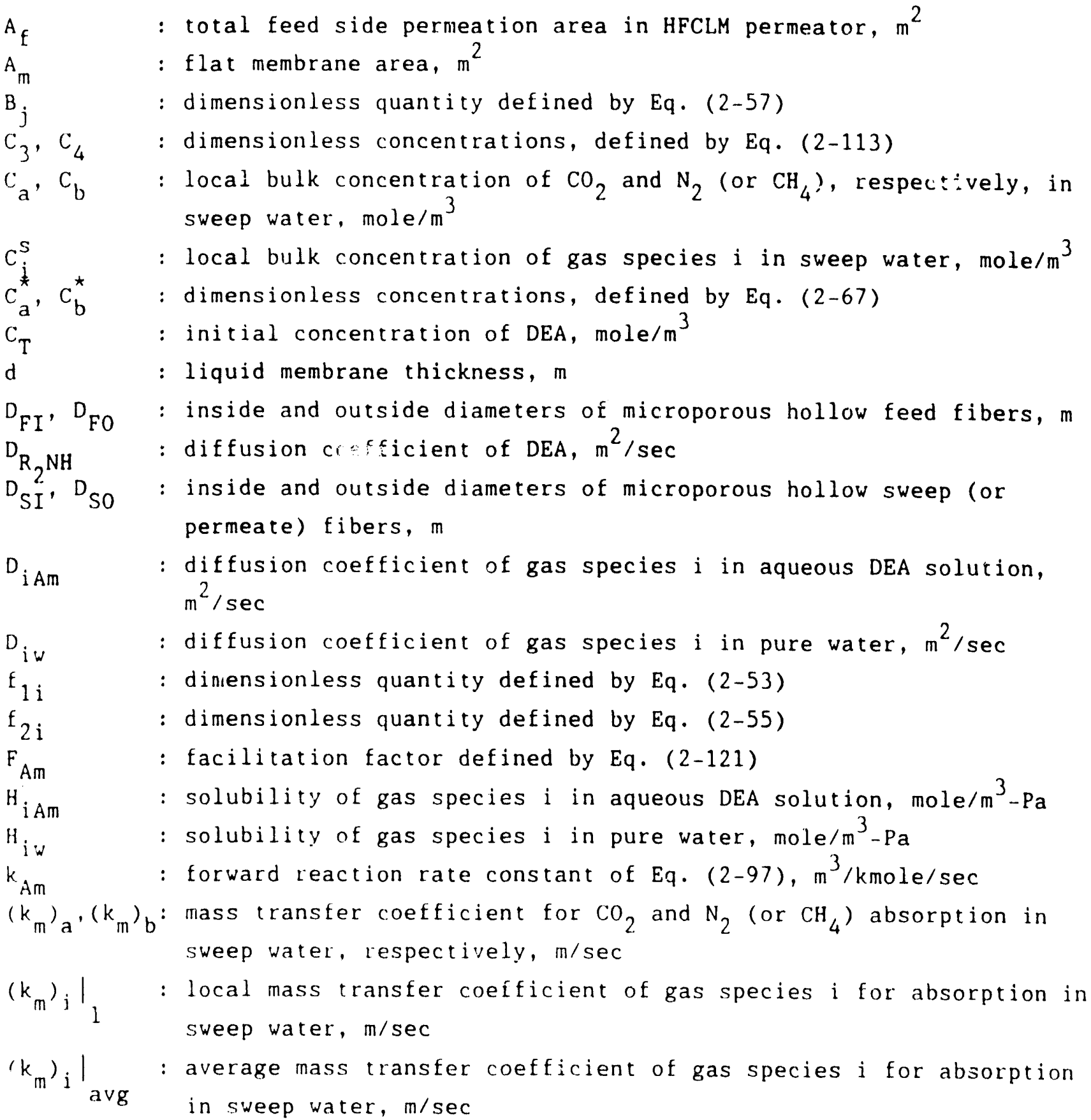




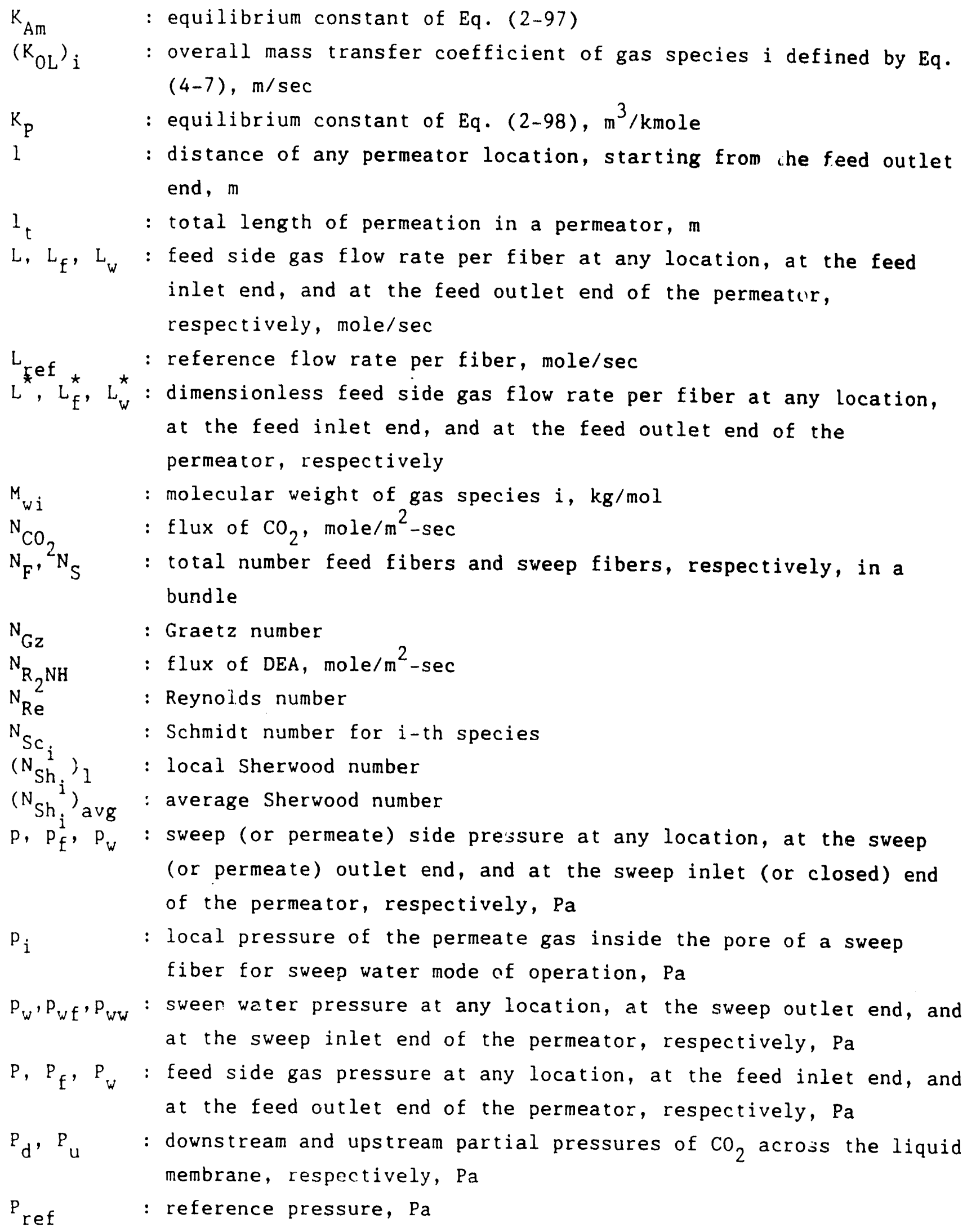




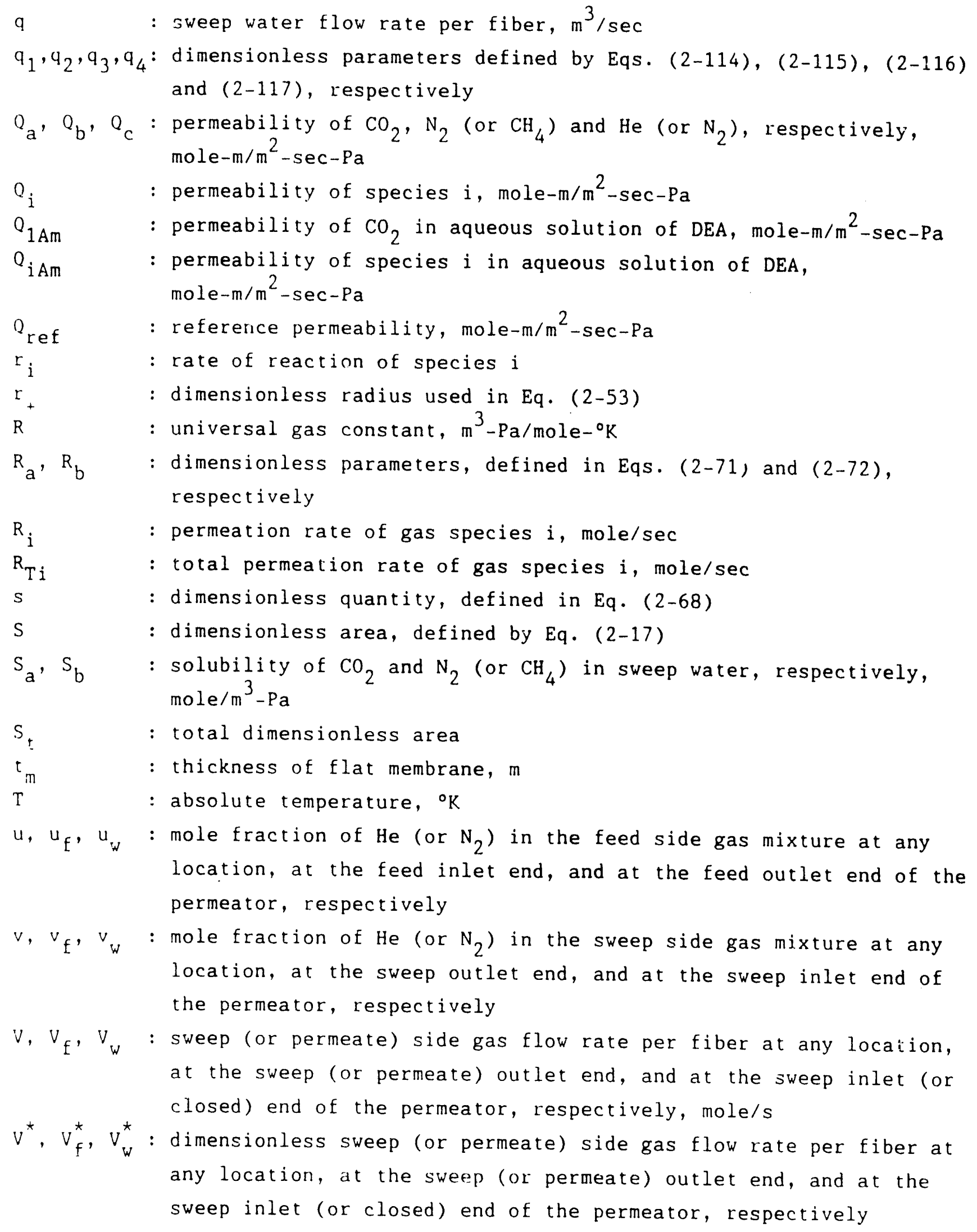




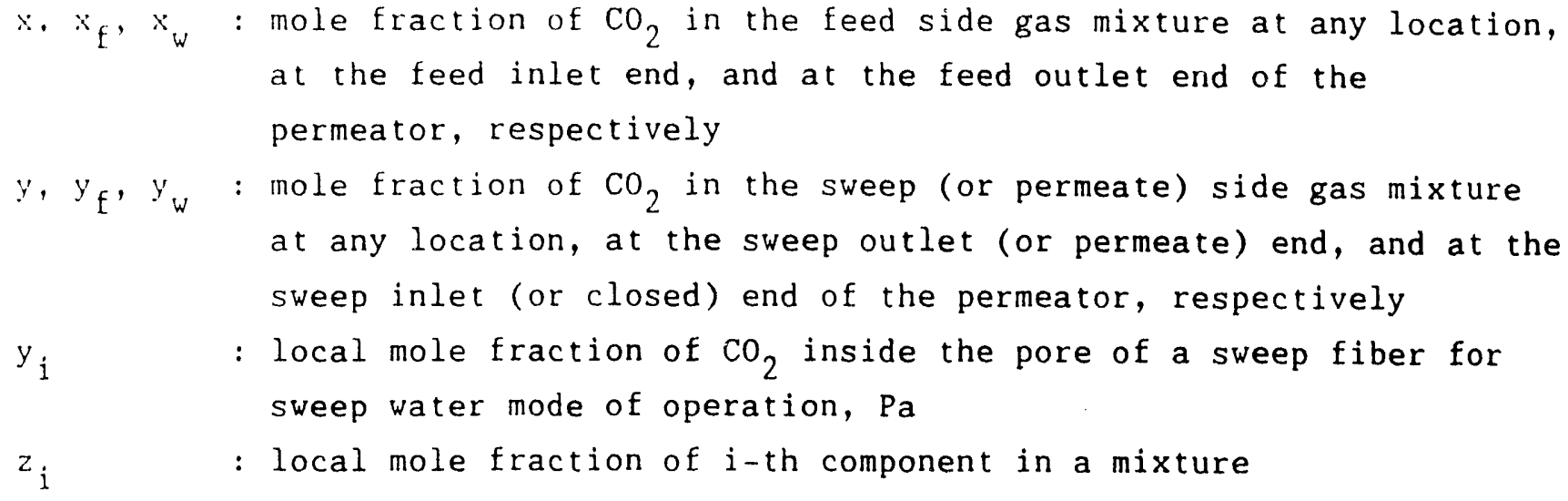

\section{Greek Symbols}

$\Psi_{1}, \Psi_{2} \quad$ : dimensionless parameters, defined in Eqs. (2-79) and (2-80), respectively

$\alpha \quad$ : dimensionless quantity, defined in Eq. (2-68)

$\alpha_{a}, \alpha_{b}, \alpha_{c}$ : dimensionless permeabilities of $\mathrm{CO}_{2}, \mathrm{~N}_{2}\left(\right.$ or $\mathrm{CH}_{4}$ ), and $\mathrm{He}$ (or $\mathrm{N}_{2}$ ), respectively, defined by $\mathrm{Eq} \cdot(2-21)$

$\alpha_{i j} \quad:$ selectivity between species $i$ and $j$, defined by Eq. (4-8)

$\beta \quad$ : dimensionless parameter, defined in Eq. (2-22)

$\beta_{F}, \beta_{S} \quad:$ dimensionless parameters, defined in Eqs. (2-69) and (2-70), respectively

$\beta_{j} \quad$ : dimensionless parameter, defined in Eq. (2-56)

$\xi \quad:$ dimensionless membrane thickness, defined in Eq. (2-113)

$r_{1}, r_{2} \quad$ : dimensionless feed gas pressure and sweep gas (or permeate) pressure, respectively, defined by Eq. (2-18)

$r_{W}, r_{i} \quad:$ dimensionless pressures, defined by Eq. (2-66)

$r_{1 f}, r_{1 w}$ : dimensionless pressures at feed gas inlet end and feed gas outlet end, respectively

$r_{2 f}, r_{2 w}$ : dimensionless pressures at sweep gas (or permeate) outlet end and sweep gas inlet (or closed) end, respectively

$r_{w f}, r_{W W}:$ dimensionless pressures at sweep water outlet end and sweep water inlet end, respectively

$\left(\Delta C_{a \vee g}\right) i \quad$ : area averaged concentration difference of $\mathrm{i}$-th species, mole $/ \mathrm{m}^{3}$ defined by Eq. (4-7)

$\Delta \mathrm{P}_{\mathrm{i}} \quad:$ partial pressure difference of $i-t$ th species, $\mathrm{Pa}$

$\varepsilon, \varepsilon_{\mathrm{m}} \quad:$ hollow fiber and flat film porosities 


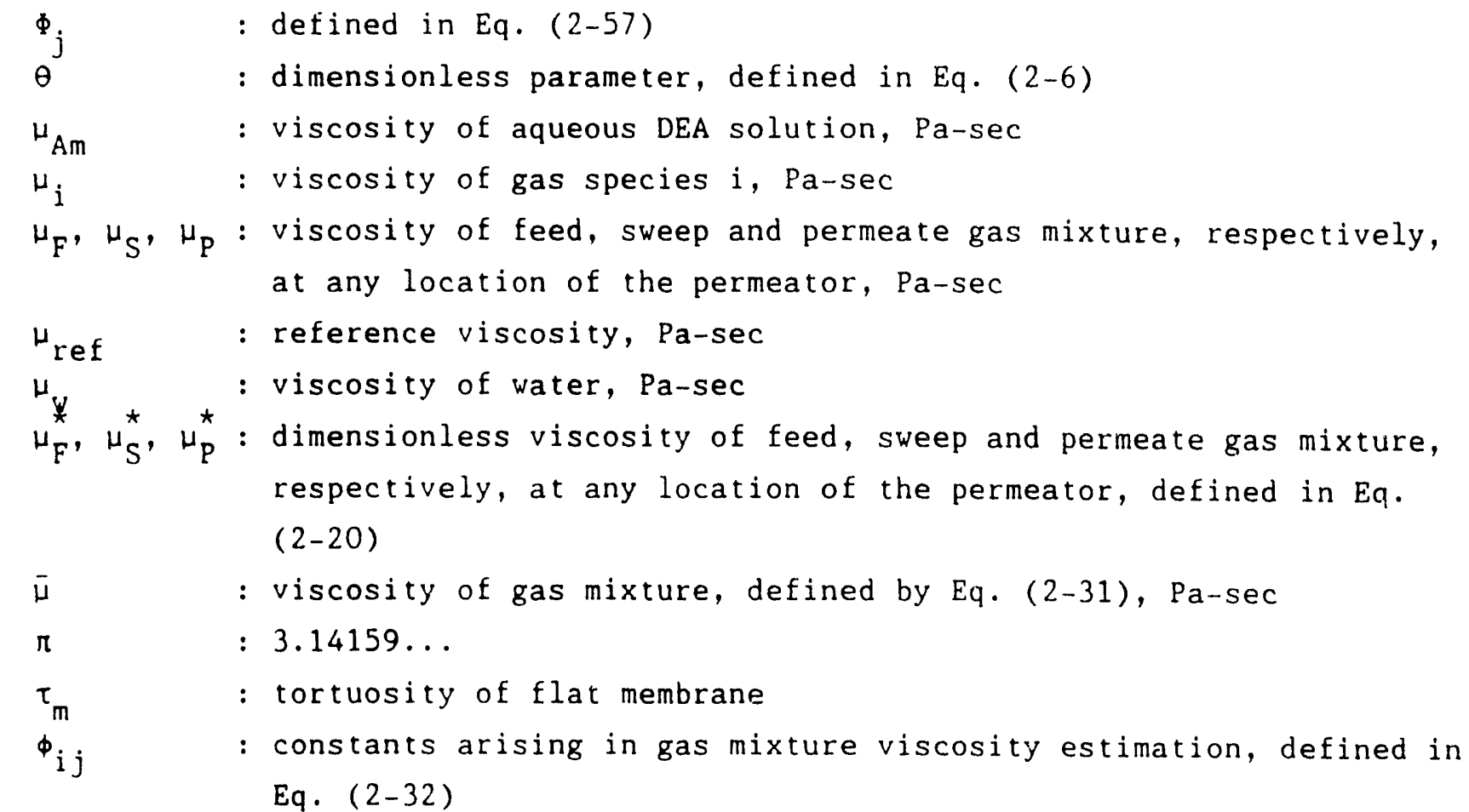

Eq. $(2-32)$ 


\section{Section 8}

\section{REFERENCES}

Astarita, G., D. W. Savage and A. Bisio, Gas Treating with Chemical Solvents, McGraw-Hill, Inc., New York (1983)

Bhave, R. R. and K. K. Sirkar, "Gas Permeation and Separation by Aqueous Membranes Immobilized Across the Whole Thickness or in a Thin Section of Hydrophobic Microporous Celgard Films," J. Membrane Sci., 27, 41 (1986)

Blanc, C. and G. Deinarais, "The reaction rate of $\mathrm{CO}_{2}$ with diethanolamine," Int. Chem. eng., 24, No. 1, 43 (1984)

Blauwhoff, P. M. M., G. F. Versteg, and W. P. M. van Swaaij, "A Study on The Reaction between $\mathrm{CO}_{2}$ and Alkanolamines in Aqueous Solutions," Chem. Eng. Sci., 38,1411 (1983)

Carey, G. F. and B. A. Finlayson, "Orthogonal Collocation on Finite Elements," Chem. Eng. Sci., 30, 587 (1975)

Chow, C. Y., An Introduction to Computational Fluid Mechanics, John Wiley and Sons, Inc., New York (1979)

Danckwerts, P. V. and M. M. Sharma, "The Absorption of Carbon Dioxide Into Solutions of Alkalis and Amines (With Some Notes on Hydrogen Sulphide and Carbonyl Sulphide)," The Chemical Engineer, CE244 (1966).

Donaldson, T. L. and Y. N. Nguyen, "Carbon Dioxide Reaction Kinetics and Transport in Aqueous Amine Membranes," Ind. Eng. Chem. Fundam., 19, 260 (1980)

Donaldson, T. L. and J. A. Quinn, "Carbon Dioxide Transport Through Enzymatically Active Synthetic Membranes," Chem. Eng. Sci., 30, 103 (1975)

Evans, F. L., Equipment Design Handbook for Refineries and Chemical Plants, 2nd Ed., Gulf Publishing Co. (1979) 
Friedlander, S. K. and K. H. Keller, "Mass Transfer in Reacting Systems near Equilibrium; Use of the Affinity Function," Chem. Eng. Sci., 20, 121 (1965)

Goddard, J. D., J. S. Schultz and R. J. Bassett, "On Membrane Diffusion with Near Equilibrium Reaction," Chem. Eng. Sci., 25, 665 (1970)

Guha, A. K., "Studies on Different Gas Separation Modes with Hollow Fiber Contained Liquid Membrane," Ph.D. Diss., Stevens Inst. of Technol., Hoboken, NJ (1989)

Hoofd, L. and F. Kruezer, "The mathematical treatment of steady state diffusion of reacting species," AIChE Symp. Ser., 77, 123 (1981)

Jackson, K. R., W. H. Enright and T. E. Hull, "User's Guide for DVERK - A Subroutine for Solving Non-Stiff ODE'S," TR No. 100, Department of Computer Science, University of Toranto (Oct. 1976)

Jain, R. and J. S. Schultz, "A Numerical Technique for Solving Carrier-Mediated Transport Problems," J. Membrane Sci., 11, 79 (1982)

Kruezer, F. and L. J. Hoofd, "Facilitated Diffusion of 0xygen in the Presence of Hemoglobin," Respir. Physiol., 8, 380 (1970)

Kutchai, H., J. A. Jacquez and F. J. Mather, "Nonequilibrium Facilitated Oxygen Transport in Hemoglobin Solutions," Biophys. J., 10, 38 (1970)

Majumdar, S., "Studies on Permeate Recycle for Producing Oxygen Enriched Air," M.S. Thesis, Stevens Inst. of Technol., Hoboken, NJ (1982)

Majumdar, S., "A New Liquid Membrane Technique for Gas Separation," Ph.D.

Diss.. Stevens Inst. of Technol., Hoboken, NJ (1986)

Majumdar, S., A. K. Guha and K. K. Sirkar, "A New Liquid Membrane Technique for Gas Separation," AIChE J., 34, 1135 (1988) 
Majumdar, S., A. K. Guha, Y. T. Lee and K. K. Sirkar, "A Two Dimensional Analysis of Membrane Thickness in a Hollow Fiber Contained Liquid Membrane Permeator," J. Mem. Sci., 43, 259 (1989)

Matley, J., Ed., Modern Cost Engineering, Vol 1, McGraw-Hill, Inc., New York (1977)

Matley, J., Ed., Modern Cost Engineering, Vol 2, McGraw-Hill, Inc., New York (1984)

Nedelman J. and S. I. Rubinow, "Facilitated Diffusion of Oxygen and Carbon monoxide in the Large Affinity Regime," J. Math. Biol., 12, 73 (1981)

NYSERDA Report 87-10, "A New Liquid Membrane Technique to Purify Landfill Gas," New York State Energy Research and Development Authority, Albany, New York (1987)

Otto, N. C. and J. A. Quinn, "The Facilitated Transport of Carbon Dioxide Through Bicarbonate Solutions," Chem. Eng. Sci., 26, 949 (1971)

Pereyra, V., "PASVA3: An Adaptive Finite Difference FORTRAN Program for First Order Nonlinear Ordinary Boundary Problems," Lecture Notes on Computer Science, Vol. 76, p. 67, Springer-Verlag, Berlin (1978)

Peters, M. S. and K. D. Timmerhaus, Plant Design and Economics for Chemical Engineers, 3rd Edition, McGraw-Hill, Inc., New York (1980)

Reid, R. C., J. M. Prausnitz and T. K. Sherwood, The Properties of Gases and Liquids, 3rd Edition, McGraw-Hill, Inc., New York (1977)

Sada, E., H. Kurmazawa and M. Butt, "Solubilities of Gases in Aqueous Solutions of Amine," J. Chem. Eng. Data, 22, No. 3, 277 (1977)

Sada, E., H. Kurmazawa and M. Butt, "Solubility and Diffusivity of Gases in Aqueous Solutions of Amine," J. Chem. Eng. Data, 23, No. 22, 161 (1978) 
Sengupta, A., "Investigation on Binary and Ternary Gas Separations Using Polymeric Membrane Permeators," Ph.D. Diss., Stevens Inst. of Technol., Hoboken, NJ (1985)

Sengupta, A. and K. K. Sirkar, "Membrane Gas Separation," in Progress in Filtration and Separation, R. J. Wakeman, Ed., Vol. 4, p. 289, Elsevier, Amsterdam (1986)

Skelland, A. H. P., Diffusional Mass Transfer, 162, Wiley, New York (1974)

Smith, K. A., J. H. Meldon and C. K. Colton, "An Analysis of CarrierFacilitated Transport," AIChE J., 19, 102 (1973)

Smith, D. R. and J. A. Quinn, "The Prediction of Facilitation Factors for Reaction Augmented Membrane Transport," AIChE J., 25, 197 (1979)

Stern, S. A., F. J. Onorato and C. Libove, "The Permeability of Gases Through Hollow Silicone Rubber Fibers: Effect of Fiber Elasticity on Gas Permeability," AIChE J., 23, 567 (1977)

Suchdeo, S. R. and J. S. Schultz, "The Permeability of Gases Through Reacting Solutions: The Carbon Dioxide-Bicarbonate Membrane System," Chem. Eng. Sci., $29,13(1974)$

Thomas, W. J. and I. A. Furzer, "Diffusion Measurements in Liquids by the Gouy Method," Chem. Eng. Sci., 17, 115 (1962)

Thorman, J. M. and S. T. Hwang, "Compressible Flow in Permeable Capillaries Under Deformation," Chem. Eng. Sci., 33, 15 (1978)

Ward, W. J., "Analytical and Experimental Studies of Facilitated Transport," AIChE J., 16, 405 (1970)

Wilke, C. R., "A Viscosity Equation for Gas Mixtures," J. Chem. Phys., 18, 517 (1950) 

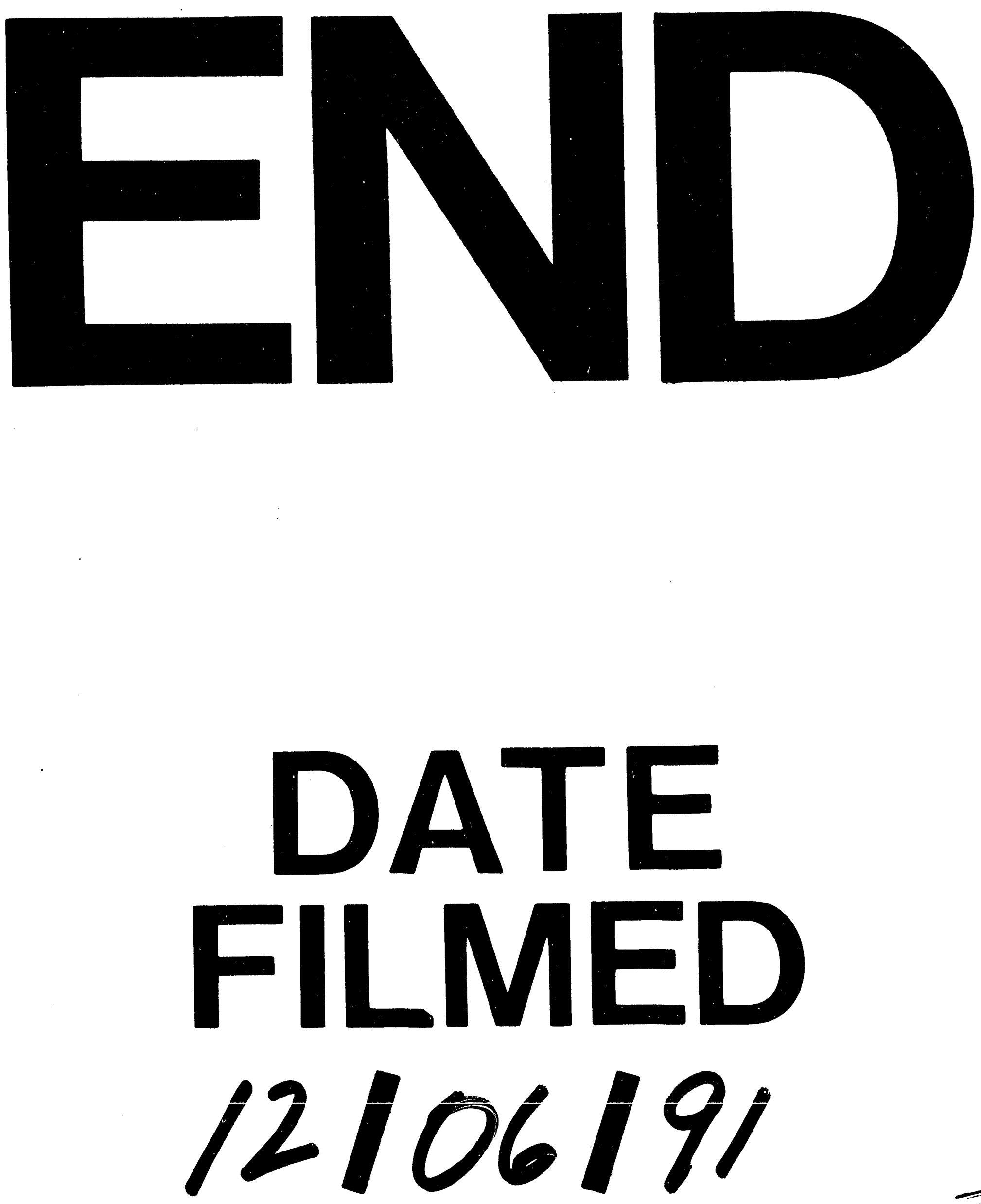
